\title{
CHECKLIST OF WOODY PLANTS OF SULAWESI, INDONESIA
}

\author{
P.J.A. KEßLER ${ }^{1}$, M.M. BOS ${ }^{1}$, S.E.C. SIERRA DAZA ${ }^{1}$, A. KOP ${ }^{1}$, \\ L.P.M. WILLEMSE ${ }^{1}$, R. PITOPANG ${ }^{2}$ \& S.R. GRADSTEIN ${ }^{3}$
}

\section{INTRODUCTION}

Sulawesi comprises of about $182,870 \mathrm{~km}^{2}$ of land and fewer botanical specimens (about 23 specimens per $100 \mathrm{~km}^{2}$, Whitten et al., 1987) have been collected here than in any other major island in Indonesia. This island is up to date botanically poorly explored and according to Van Steenis (1950) about 32,500 specimens of plants wererecorded, the number is probably only a rough estimation and certainly in the precomputer era not based on real specimens. Within the framework of STORMA (Stability of Rainforest Margins) we analyzed the vegetation in several plots of one hectare of different land use systems at the Lore Lindu National Park, Central Sulawesi, Indonesia. Rapidly we were faced with the problem that the identification of the mostly sterile trees would be almost impossible without a sound basic checklist. Because previous hardcopies (Hildebrand, 1950; Soewanda \& Tantra, 1973; Whitmore et al., 1989) were either outdated or incomplete (especially for the small diameter trees) we decided to create a new one using data of actual plant specimens housed at the National Herbarium of the Netherlands, Universiteit Leiden branch (with duplicate specimens in several other herbaria like BO, E, $\mathrm{K}$ and others), and enter them in the BRAHMS (Botanical Research and Herbarium Management Systems) database developed by Denis Filer, University of Oxford.

More than 120 woody families have been screened and the label information of all specimens (c. 13,000) checked and partly analysed. The density index calculated from our figures is very low (7) but for the first time underpinned by hard, retrievable data. As we did not include species - and specimens rich herbaceous families in our calculations (Orchidaceae, Poaceae, Zingiberaceae, ferns, etc.) the much lower collection index can be at least partially explained.

1) Nationaal Herbarium Nederland, Universiteit Leiden branch, P.O. Box 9514, 2300 RA Leiden, The Netherlands; e-mail: Kessler@nhn.leidenuniv.nl

2) Universitas Tadulako, Faculty of Agriculture, Kampus Bumi Tondo, Palu, Sulteng, Indonesia; e-mail: Pitopang64@yahoo.com

3) Albrecht von Haller Institute of Plant Sciences, Department of Systematic Botany, Untere Karspüle 2, 37073 Göttingen, Germany; e-mail: Sgradst@gwdg.de 


\section{FORMAT}

The hardcopy of the checklist has been prepared from the specimen database, with some editing in word-processing and has the following format:

Example:

ACERACEAE (Family name in alphabetical order)

ACER (Genus name in alphabetical order)

Acer laurinum Hassk. (Scientific name in alphabetical order)

Vernacular name(s) - Dogo (Central Sulawesi)

Sulawesi distribution - North (S. Utara) Central (S. Tengah), South (S. Selatan), and South-East (S. Tenggara) (Distribution in Sulawesi on old province level)

Lore Lindu National Park \& environs - present (Recorded for Lore Lindu National Park and environs; the area comprises the coordinates $119^{\circ} 30^{\prime} \mathrm{E}-120^{\circ} 50^{\prime} \mathrm{E}$ and $2^{\circ} 00^{\prime} \mathrm{S}-0^{\circ} 30^{\prime} \mathrm{S}$ )

Altitude $-750-2550 \mathrm{~m}$

Collection(s) — De Vogel 2341; Keßler PK 2005; Ramadhanil 503, 607 (all collections present at L)

The database proper comprises many more detailed fields and thus information which can fully explored only with the aid of a personal computer. In a later stage a searchable website on the internet is envisaged.

\section{ANALYSES}

Some preliminary analyses of the overall data revealed that the Euphorbiaceae with about 1600 records followed by Rubiaceae (c. 730 records) and Myrtaceae (c. 630 records) represent the three plant families with the highest number of collections. These results may be due to collection artefacts as almost all these taxa are relatively small, regularly flowering and species rich families in the understorey of the tropical rain forest. Large trees that are flowering and fruiting in irregular intervals are underrepresented like Ebenaceae (c. 90 records), Combretaceae (c. 70 records) or Dipterocarpaceae (c. 70 records).

Van Steenis (1950) listed c. 150 plant collectors who worked in Sulawesi and neighbouring islands, the botanical exploration starting with Dampier (1687) who collected on the island of Buton. Within the last 50 years some important fieldtrips were organised by the Royal Botanic Gardens Kew (Coode et al.), Royal Botanic Garden Edinburgh (Argent, Newman, Milliken, Atkins et al.), the Nationaal Herbarium Nederland (formerly the Rijksherbarium: De Vogel et al., Hennipman, Joncheere, Keßler, Van Balgooy, Vermeulen), and Naturalis (to which Coode of the Royal Botanic Gardens, Kew attached himself) with the collaboration of either Universitas Tadulako (Ramadhanil Pitopang et al.), Herbarium Bogoriense (Mogea, Tantra, Wiriadinata et al.) and/ 
or Kebun Raya Bogor (Dedi Darnaedi, Hendrian, Inggit et al.) These recent collections considerably improved our knowledge of the floristic composition of this island.

Collection sites are not very well spread among the island and the number of collections from Lore Lindu National Park is relatively low, mainly due to the difficult access in former times. As far as possible, longitude and latitude data of localities of historical collections have been added using gazetteer records in order to delineate the past and present distribution of species within the island.

The elevation data are relatively weak as almost all historical collections lack detailed information on altitude. Only recently more details have been noted on the specimen labels.

Our data demonstrate that extensive routine collecting work is still necessary and should be given priority as the (lowland) primary forest may be gone within a decade. We therefore are fully aware that the present data and specimens available represent only a fraction of the rich flora of Sulawesi either in number of specimens or in number of species. It is, however, envisaged to enrich the present data considerably in the near future by more new collections. Further improvement could be reached if the vast collection information from Sulawesi specimens of several other herbaria, especially those of Herbarium Bogoriense could also be entered into a database and linked to the existing one.

Although the checklist was formulated as a book project (in order to reach the target groups) we will also try to put the rich information in a searchable database on internet. We hope that in this way our data can be used by those users who need fast and reliable information especially within the nature conservation community.

\section{ACKNOWLEDGEMENTS}

We wish to thank all specialists who kindly helped us in the identification of our own collections from Sulawesi, especially:

F.A.C.B. Adema (Leguminosae p.p., Sapindaceae p.p.), M.M.J. van Balgooy (preidentification), Ding Hou (Anacardiaceae, Celastraceae, Leguminosae p.p., Rhizophoraceae), H.P. Nooteboom (Magnoliaceae, Simaroubaceae), C. E. Ridsdale (Rubiaceae), W. Vink (Sapotaceae), P.C. van Welzen (Euphorbiaceae, Sapindaceae p.p.), E.F. de Vogel (Orchidaceae), and W.J.J.O. de Wilde (Cucurbitaceae, Myristicaceae). The herbarium management staff at L kindly provided extra assistance.

Various institutions and colleagues provided us with extra data and information:

M.J.E. Coode (K, collection list from Sulawesi, general information), J. Dransfield (K, list of palms collected by Musser), H. Atkins (E, collection list from Sulawesi), E. Ashley (NY, Sulawesi records from their database). The late T.C. Whitmore identified several Macaranga specimens.

Last but not least Denis Filer (OXF) supported us in preparing modules to retrieve the necessary data out of the BRAHMS database in a suitable format for printing. 


\section{LITERATURE}

Hildebrand, F.H. 1950. Daftar nama pohon-pohonan Selebes. Laporan Balai Penelitian Kehutanan 43: $1-105$.

Soewanda, A.P. \& I.G.M. Tantra. 1972. Daftar nama pohon-pohonan, repisi I Sulawesi Selatan, Tenggara dan sekitarnya. Laporan Lembaga Penelitian Hutan 151: 1-113.

Van Steenis, C.G.G.J. 1950. Flora Malesiana I, 1, Noordhoff-Kolff, Jakarta.

Whitmore, T.C., et al. 1989. Tree flora of Indonesia, Checklist for Sulawesi. Forest Research and Development Centre, Bogor.

Whitten, A.J., et al. 1987. The ecology of Sulawesi. Gadjah Mada University Press, Yogyakarta. 


\section{CHECKLIST}

ACERACEAE

ACER

Acer laurinum Hassk.

Sulawesi distribution - Central, South

Altitude - 700-1850 m

Collection(s) - bb 19707, 20229, 20882, 28213;

Bünnemeyer 12347, 12407; Kjellberg 2951;

Koorders 18816; Meijer 11025

\section{ACTINIDIACEAE}

\section{SAURAUIA}

\section{Saurauia altissima Zipp.}

Sulawesi distribution - North

Altitude - 500-700 m

Collection(s) - Koorders 18953, 18956, 19297, 19301

Saurauia bracteosa DC.

Sulawesi distribution - South

Altitude $-2200 \mathrm{~m}$

Collection(s) - Bünnemeyer 11834

Saurauia callithrix Miq.

Sulawesi distribution - North

Altitude - 400-700 m

Collection(s) - Koorders 18932, 18936, 19295

Saurauia costata Reinw. ex de Vriese

Sulawesi distribution - North, Central

Altitude - 750-1550 m

Collection(s) - Forsten 638; Koorders 18954, 19293; Meijer 9345

Saurauia euryolepis de Vriese

Sulawesi distribution - North

Altitude - 500-900 m

Collection(s) - Forman 197; Koorders 18943 , 18949,18958

Saurauia hoeveniana Koord.

Sulawesi distribution - North

Altitude - 450-600 m
Collection(s) - Koorders 18944, 18947, 19283

Saurauia javanica (Nees) Hoogland

Sulawesi distribution - North

Altitude $-400 \mathrm{~m}$

Collection(s) - Lam 2413

\section{Saurauia lepidocalyx Miq.}

Sulawesi distribution - North

Altitude - 317-1000 m

Collection(s) - Alston 16247; De Vogel 2462, 2508; De Vriese \& Teijsmann 113; Kaudern 15; Koorders 18933, 18941, 22619, 24069; Teijsmann HB 5282, HB 5289, HB 5733

Saurauia minahassae Koord.

Sulawesi distribution - North, Central

Altitude $-0-600 \mathrm{~m}$

Collection(s) - Burley, Partomihardjo \& Rachman 3537; Koorders 19284, 19285

Saurauia oligolepis Miq.

Sulawesi distribution - North, Central, South Altitude - 450-1700 m

Collection(s) - De Vogel 5595; Koorders 18931, 19294, 19296; Meijer 9260, 9419, 9844, 10147; Van Balgooy 3021, 3035, 3059, 4025

Saurauia oligolepis Miq. var. squamosa Koord.

Sulawesi distribution - North

Altitude $-120 \mathrm{~m}$

Collection(s) - Koorders 19282

Saurauia polyodon Miq.

Sulawesi distribution - North

Altitude $-600 \mathrm{~m}$

Collection(s) - Kaudern 20

Saurauia schwarzii Koord.

Sulawesi distribution - North

Altitude - $700 \mathrm{~m}$

Collection(s) - Koorders 18948

Saurauia spadicea Blume

Sulawesi distribution - North

Altitude $-1100 \mathrm{~m}$ 
Collection(s) - Alston 15912; Forman 376

Saurauia tristyla DC. forma celebica Miq.

Vernacular name(s) - Kapapojan

Sulawesi distribution - North

Altitude - 40-500 m

Collection(s) - De Vogel 2576; Koorders 18942, 18951, 18959, 22622; Lam 2424

Saurauia vanderhoeveniana Koord.

Sulawesi distribution - North

Altitude - 40-1000 m

Collection(s) - De Vogel 2511, 2544

Saurauia wawaroentoi Koord.

Sulawesi distribution - North

Altitude $-800 \mathrm{~m}$

Collection(s) - Koorders 18957

Saurauia wigmanni Koord.

Sulawesi distribution - North

Altitude $-0-500 \mathrm{~m}$

Collection(s) - Koorders 18946, 18955

Saurauia spec.

Vernacular name(s) - Gurala'a; Paredeang; Sisup sopun [Tombulu ?]

Sulawesi distribution - North, Central, South, South-East

Lore Lindu National Park \& environs - present

Altitude - 0-2900 m

Collection(s) - Alston 15707, 15728, 16150, 16266; Amir 187; bb 23905, 24100, 24189; Bloembergen 4006, 4130, 4133, 4226, 4256; Cel II 300; Coode 5905, 5963, 6064, 6208, 6271, 6283; De Vogel 2482, 5163A, 5186, $5284,5312,5510,5570,6011,6013,6014$, 6076; De Vogel \& Vermeulen 6765, 6767, 6891; Elbert 2793, 2824, 2874, 2918, 3021, 3148, 6634, 6709, 6710, 6765; Eyma 414, $450,465,969,998,1127,1148,1686,1708$, 3466,4158 , 4183; Forman 187, 301; Hennipman 5499, 5644, 5690, 5978, 6087; Johansson 171, 342; Keßler et al. PK 3141; Kofman 215; Lam 2638, 2754, 2883; Meijer 9370, 9385, 9482a, 9521, 9761, 10025; Noerkas 345; Prawiroatmodjo \& Maskur 1281; Prawiroatmodjo \& Soewoko 1720; Ramlanto 250, 258; Ramlanto \& Fanani 462; Sands 212, 407, 532; Van Balgooy 3038, 3188, 3473, $3511,3818,3986$; Whitmore \& Sidiyasa
TCW 3513, TCW 3537, TCW 3546; Yoshida 1332

\section{ALANGIACEAE}

ALANGIUM

Alangium griffithii (C.B. Clarke) Harms

Vernacular name(s) - Manata

Sulawesi distribution - Central, South

Altitude $-1000 \mathrm{~m}$

Collection(s) - Cel V 128; Van Balgooy 3583

Alangium javanicum (Blume) Wang

Vernacular name(s) - Lansabonti

Sulawesi distribution - North, Central, South Altitude - 150-700 m

Collection(s) - bb 17056; Burley, Partomihardjo \& Rachman 3929; Cel V 199; Koorders 20844; Ramlanto \& Fanani 676, 717

Alangium maliliense Bloemb.

Sulawesi distribution - South

Altitude $-300 \mathrm{~m}$

Collection(s) - Cel 161

Alangium salvifolium (L.f.) Wang subsp. sundanum (Miq.) Bloemb.

Sulawesi distribution - South

Collection(s) - Noerkas 287; Teijsmann HB 11849 , HB 11894, HB 12117, HB 12436, HB 13832

\section{Alangium spec.}

Sulawesi distribution - North

Altitude $-400 \mathrm{~m}$

Collection(s) - Koorders 16380; Whitmore \& Sidiyasa TCW 3534

\section{ANACARDIACEAE}

\section{ANACARDIUM}

\section{Anacardium occidentale L.}

Sulawesi distribution - North, Central, SouthEast

Altitude - 0-200 m

Collection(s) - Elbert 3306; Rachmat 198, 619 
BUCHANANIA

Buchanania arborescens (Blume) Blume

Vernacular name(s) - Bulo Bulo [Kendari]; Kapofo; Lokinako; Marisin karea'a; Morontoboea; Ninifo; Taniruan'a

Sulawesi distribution - North, Central, South, South-East

Altitude - 0-600 m

Collection(s) - bb 3919, 6648, 13750, 24710, 31845, 31899; Bünnemeyer 10777; Cel II 406; De Vogel 6031, 6053, 6065, 6186, 6264, 6376; Elbert 3421; Koorders 15941, 15943; Lam 2448, 2577, 2617, 2920, 3292; Meijer 10185, 11185; Prawiroatmodjo \& Soewoko 1724; Ramlanto 140; Van Balgooy 3755, 3793; Whitmore \& Sidiyasa TCW 3525

\section{CAMPNOSPERMA}

\section{Campnosperma auriculatum Hook.f.}

Sulawesi distribution - South

Altitude $-100 \mathrm{~m}$

Collection(s) - Van Balgooy 3781

\section{Campnosperma brevipetiolatum Volkens}

Vernacular name(s) - Dalipo; Taniruan'a

Sulawesi distribution - North, South, SouthEast

Altitude - 10-150 m

Collection(s) - bb 21737; Cel II 329; Lam 2769

\section{DRACONTOMELON}

Dracontomelon dao (Blume) Merr. \& Rolfe

Vernacular name(s) - Boech rao, Woea raoe; Kaili; Lolomas; Rago; Rao

Sulawesi distribution - North, Central, South, South-East

Altitude - 0-257 m

Collection(s) - bb 4994, 15386, 17641, 21334, 22955, 28728, 32361, 32619; Burley, Partomihardjo \& Rachman 4231; Cel I 9, IV 113; Chin 3435; De Vogel 2446; De Vogel \& Vermeulen 6485, 6651, 6654; Elbert 2632; Koorders 15945, 15946, 15947, 15948, 15949, 15951, 15959, 15964, 16329, 16331, 19619; Meijer 9222, 10066A, 10758; Noerkas 204; Teijsmann HB 11807, HB 12853; Whitmore \& Sidiyasa TCW 3423

\section{GLUTA}

\section{Gluta papuana Ding Hou}

Vernacular name(s) - Nato [Tator]

Sulawesi distribution - South

Altitude - $400 \mathrm{~m}$

Collection(s) - De Vogel 5705, 5796, 5994, 6004; Van Balgooy 3780

\section{Gluta renghas L.}

Vernacular name(s) - Belag / Sanga / Anga [Bugin / Makas / Toki]; Poei-poei

Sulawesi distribution - South, South-East

Altitude - 1-50 m

Collection(s) - bb 19693, 21127, 23304, 23305, 23306, 32370, 32469, 32609; Cel III 79, III 139; Kaudern 524

\section{KOORDERSIODENDRON}

Koordersiodendron pinnatum (Blume) Merr.

Vernacular name(s) - Boegis; Buwis'a; Goei; Lojow [Kotamobagu]; Oeris; Ore

Sulawesi distribution - North, Central, South, South-East

Altitude - 10-800 m

Collection(s) — bb 4180, 5571, 19606, 23903, 26265, 26266, 26267, 28731, 31492, 31838, 32362, 32483, 32617; Burck 1430; Burley, Partomihardjo \& Rachman 4174, 4275; Cel I 6, V 149, V 347; De Vogel \& Vermeulen 6550, 6653; Ham L 2; Koorders 15955, $15957,15958,15960,15963,15968,15969$, 15970, 16328, 18849, 19711; Lam 2944, 3177; Whitmore \& Sidiyasa TCW 3452

\section{MANGIFERA}

\section{Mangifera celebica Kosterm.}

Vernacular name(s) - Loemisi

Sulawesi distribution - South

Collection(s) - Cel II 453; Teijsmann HB 12562, HB 12683

\section{Mangifera foetida Lour.}

Vernacular name(s) - Mangga hoetan/ Oemba; Mangga Itam [Kotamobagu]; Ontjoh

Sulawesi distribution - North, Central, South Altitude - 40-1000 m 
Collection(s) - bb 29479; Cel V 165; De Vogel \& Vermeulen 6606; Meijer 9610

\section{Mangifera leiophylla Kosterm.}

Sulawesi distribution - North

Altitude $-700 \mathrm{~m}$

Collection(s) - De Vogel \& Vermeulen 6782

\section{Mangifera minor Blume}

Vernacular name(s) - Kajoe taipa dare; Taipa dondri

Sulawesi distribution - Central, South

Lore Lindu National Park \& environs - present

Altitude $-0-900 \mathrm{~m}$

Collection(s) - bb 21 103, 21104; Bünnemeyer 11565; Cel I 26; De Vogel 5040; Elbert 2638, 2729; Eyma 3805

\section{Mangifera pedicellata Kosterm.}

Sulawesi distribution - South

Collection(s) - Wirawan 1

\section{Mangifera quadrifida Jack}

Sulawesi distribution - North, South

Altitude - 50-150 m

Collection(s) - bb 19439; Cel II 123; Meijer 10762

\section{Mangifera sulavesiana Blanco}

Vernacular name(s) — Morotoipa; Tamba

Sulawesi distribution - South

Collection(s) - Cel II 448, II 473

\section{Mangifera spec.}

Vernacular name(s) - Ansam [Luwuk] Sulawesi distribution - Central, South Altitude - 1000-1300 m

Collection(s) - Keßler et al. PK 3125; Meijer 9484, 10762

\section{RHUS}

Rhus taitensis Guillaumin

Vernacular name(s) - Nanitu

Sulawesi distribution - North, Central

Lore Lindu National Park \& environs - present

Altitude $-100 \mathrm{~m}$

Collection(s) — Eyma 3453; Koorders 15950, 16297, 18898; Lam 2572

\section{SEMECARPUS}

Semecarpus acida Blume

Sulawesi distribution - North

Altitude - $500 \mathrm{~m}$

Collection(s) - Burley, Partomihardjo \& Rachman 3806

\section{Semecarpus cuneiformis Blanco}

Vernacular name(s) - Sibotoe

Sulawesi distribution - North, South, SouthEast

Altitude - 0-755 m

Collection(s) - bb 4193; De Vogel \& Vermeulen 6664, 6914; Elbert 2694, 2763, 3161, 3370, 3446; Eyma 4193; Forman 302; Koorders 15972, 15975, 19763; Rachmat 796; Teijsmann HB 12415, HB 12640, HB 14104; Whitmore \& Sidiyasa TCW 3544

\section{Semecarpus forstenii Blume}

Vernacular name(s) - Gedju warani [Kolawi] Sulawesi distribution - Central, South, SouthEast

Lore Lindu National Park \& environs - present Altitude - 2-1050 m

Collection(s) - bb 21720, 28247; De Vogel 5584; Meijer 9460

\section{Semecarpus longifolius Blume}

Sulawesi distribution - North, Central, South, South-East

Altitude - 0-400 m

Collection(s) - bb 19825; Elbert 2512, 3277; Meijer 9177, 10050; Teijsmann HB 12121

\section{Semecarpus spec.}

Vernacular name(s) - Warani [Napu]

Sulawesi distribution - Central

Altitude - 1000-1040 m

Collection(s) - Keßler et al. PK 3167; Van Balgooy 3134

\section{SPONDIAS}

\section{Spondias dulcis Parkinson}

Sulawesi distribution - South

Altitude $-300 \mathrm{~m}$

Collection(s) - Cel V 176 
Spondias malayana Kosterm.

Vernacular name(s) - Golo

Sulawesi distribution - North, South, SouthEast

Altitude - 4-900 m

Collection(s) - bb 5857, 17156; Cel V 176; Koorders 15976, 15977, 15978; Prawiroatmodjo \& Soewoko 1684,1835

Spondias philippinensis (Elmer) Kosterm.

Sulawesi distribution - North, South

Altitude $-400-550 \mathrm{~m}$

Collection(s) - Burley, Partomihardjo \& Rachman 3790; De Vogel 5950; Hennipman 5835; Meijer 11125

Spondias pinnata (L.f.) Kurz

Sulawesi distribution - South

Collection(s) - Yusuf \& Wahyono 5

\section{ANNONACEAE}

\section{ALPHONSEA}

\section{Alphonsea javanica Scheff.}

Sulawesi distribution - North, Central, South Altitude $-1000 \mathrm{~m}$

Collection(s) - Cel V 229; Darnaedi 1486; De Vogel 2604; De Vogel \& Vermeulen 6560, 6572; De Vriese 213; Johansson 537; Koorders 15979, 16029; Van Balgooy 3101, 3431; Whitmore \& Sidiyasa TCW 3545

\section{Alphonsea spec.}

Sulawesi distribution - North, Central

Lore Lindu National Park \& environs - present Altitude $-500-850 \mathrm{~m}$

Collection(s) - Burley et al. NGS/NCI 3615; Johansson JNR 400

\section{ANAXAGOREA}

\section{Anaxagorea javanica Blume}

Sulawesi distribution - Central

Altitude $-50 \mathrm{~m}$

Collection(s) - Sidiyasa \& Didi 1882

\section{Anaxagorea spec.}

Sulawesi distribution - North, Central
Altitude $-180-270 \mathrm{~m}$

Collection(s) - Coode 6026; Whitmore \& Sidiyasa TCW 3460

\section{ANNONA}

\section{Annona squamosa L.}

Sulawesi distribution - Central

Lore Lindu National Park \& environs - present

Collection(s) - Meijer 9195

\section{ARTABOTRYS}

\section{Artabotrys macrantha Holthuis}

Sulawesi distribution - North

Collection(s) - Lam 3003

Artabotrys trichopetalus Merr.

Sulawesi distribution - South

Altitude $-430 \mathrm{~m}$

Collection(s) - De Vogel 6252

\section{Artabotrys spec.}

Sulawesi distribution - South-East

Altitude $-250 \mathrm{~m}$

Collection(s) - Prawiroatmodjo \& Soewoko 1930; Van Hulstijn \& Atjeh 321

\section{CANANGA}

Cananga odorata Hook.f \& Thomson

Sulawesi distribution - North, Central, South, South-East

Altitude $-1000 \mathrm{~m}$

Collection(s) - bb II 444, 5554, 13490, 15389 , $17642,23573,28250,28720,31828$; Burley et al. 3800, 3801; De Vogel 2574; Koorders 15997, 15998, 15999, 16002; Lam 2857; Meijer 10081; Prawiroatmodjo \& Soewoko 1737; Ramlanto \& Fanani 154, 674; Van Balgooy 3044; Whitmore \& Sidiyasa TCW 3482

\section{CYATHOCALYX}

Cyathocalyx acuminatus C.B. Rob.

Sulawesi distribution - North

Altitude - 70-400 m

Collection(s) - Burley, Partomihardjo \& Rachman 3858; Lam 2738, 2926 
Cyathocalyx biovulatus Boerl.

Sulawesi distribution - North

Altitude $-350 \mathrm{~m}$

Collection(s) - Burley, Partomihardjo \& Rach$\operatorname{man} 3673$

Cyathocalyx kingii Boerl.

Sulawesi distribution - North

Altitude $-750 \mathrm{~m}$

Collection(s) - Burley et al. 3901; Koorders $15991,15996,16016,16030,16033$

Cyathocalyx minahassae Boerl.

Sulawesi distribution - North

Altitude $-1300 \mathrm{~m}$

Collection(s) - Forman 215; Koorders 15986, 16039

\section{Cyathocalyx petiolatus Diels}

Sulawesi distribution - North

Altitude - 350-750 m

Collection(s) - Burley et al. 3958

\section{Cyathocalyx spec.}

Sulawesi distribution $\rightarrow$ Central, South

Altitude $-1100 \mathrm{~m}$

Collection(s) - Cel II 375; Darnaedi 2072; De Vogel 6248; Meijer 9379

\section{DESMOS}

Desmos chinensis Lour.

Sulawesi distribution - North, South-East

Altitude - $900 \mathrm{~m}$

Collection(s) - Koorders 16008; McDonald \& Ismail 4164

\section{Desmos spec.}

Sulawesi distribution - South-East

Altitude $-20-250 \mathrm{~m}$

Collection(s) - Prawiroatmodjo \& Soewoko 1781

\section{FISSISTIGMA}

Fissistigma borneense (Miq.) Merr.

Sulawesi distribution - Central

Lore Lindu National Park \& environs — present
Altitude $-650 \mathrm{~m}$

Collection(s) - De Vogel 5627

Fissistigma kingii (Boerl.) Burkill

Sulawesi distribution - South

Altitude $-500 \mathrm{~m}$

Collection(s) - Van Balgooy 3940

Fissistigma latifolium (Dunal) Merr.

Sulawesi distribution - North

Altitude - 150-750 m

Collection(s) - Burley et al. 3907, 4049, 4167

\section{FRIESODIELSIA}

\section{Friesodielsia spec.}

Sulawesi distribution - North

Altitude $-150 \mathrm{~m}$

Collection(s) - Burley et al. 4245

\section{GONIOTHALAMUS}

\section{Goniothalamus brevicuspis Miq.}

Sulawesi distribution - North, Central, South Altitude - 350-1500 m

Collection(s) - Burley et al. 3567, 3584, 3714, 3884; Koorders 16003, 16004, 16017, 16036; Meijer 10001, 10018; Milliken 932; Ramlanto \& Fanani 471; Riedel 5832; Whitmore \& Sidiyasa TCW 3391

Goniothalamus euneurus Miq.

Sulawesi distribution - North

Collection(s) - Riedel 5817

\section{Goniothalamus majestatis Keßler}

Sulawesi distribution - Central, South-East Altitude $-900 \mathrm{~m}$

Collection(s) - Coode 6060, 6140, 6291; McDonald \& Ismail 3896

\section{Goniothalamus spec.}

Sulawesi distribution - North, Central, South Altitude $-500 \mathrm{~m}$

Collection(s) - Cel II 391; Darnaedi 2284; De Vogel 2549; De Vogel \& Vermeulen 7010; De Vriese \& Teijsmann 64; Milliken 914; Ramlanto \& Fanani 632; Sidiyasa \& Didi 1865 


\section{HAPLOSTICHANTHUS}

Haplostichanthus gamopetala (Boerl. ex Koord.) Heusden

Sulawesi distribution - North

Collection(s) - Koorders 15994, 16014

\section{MITREPHORA}

Mitrephora celebica Scheff.

Sulawesi distribution - North, South Altitude - $350 \mathrm{~m}$

Collection(s) - Bogor Botanical Garden 799C, 5787, IV.H. 51A, IV.H. 61A; Burley, Partomihardjo \& Rachman 3746; Cel V 247; De Vogel 2586; Okada 3458; Teijsmann HB 114

\section{Mitrephora diversifolia Miq.}

Sulawesi distribution - North, South-East Altitude - 0-200 m

Collection(s) - bb 21682; Elbert 3408

\section{Mitrephora ferruginea Boerl.}

Sulawesi distribution - North, Central

Altitude $-300 \mathrm{~m}$

Collection(s) - Cel V 194; Koorders 16011, 16012

Mitrephora minahassae Boerl. ex Koord.

Sulawesi distribution - North

Collection(s) - Koorders 16013

Mitrephora oxycarpa Boerl. ex Koord.

Sulawesi distribution - North

Collection(s) - Koorders 16019

\section{Mitrephora spec.}

Sulawesi distribution - North

Altitude $-220 \mathrm{~m}$

Collection(s) - De Vogel \& Vermeulen 6512

\section{OROPHEA}

\section{Orophea celebica Miq.}

Sulawesi distribution - North, Central, South, South-East

Altitude $-1000 \mathrm{~m}$

Collection(s) - bb 23906, V 172; Burley et al. 3578; Coode 5938, 6211; De Vogel \& Ver- meulen 6609; Hennipman 5552; Koorders 16007, 16027; Milliken 874, 911; Reinwardt 1525; Van Balgooy 3036; Whitmore \& Sidiyasa TCW 3416

Orophea creaghii (Ridl.) Leonardía \& Keßler

Sulawesi distribution — North

Collection(s) - bb 17165

\section{Orophea enneandra Blume}

Sulawesi distribution - North

Altitude - 200-750 m

Collection(s) - Burley, Partomihardjo \& Rachman 4127; Burley et al. 3713

Orophea hexandra Blume

Sulawesi distribution - North

Altitude $-300 \mathrm{~m}$

Collection(s) - Afriastini \& Rohajawati JJA 2932

Orophea spec.

Sulawesi distribution - North, Central

Altitude - 80-1000 m

Collection(s) - bb 17631; De Vogel 5088A; De Vogel \& Vermeulen 6998

\section{PHAEANTHUS}

Phaeanthus ebracteolatus (Presl) Merr.

Sulawesi distribution - North

Altitude $-500 \mathrm{~m}$

Collection(s) - De Vogel 2558; Forman 230; Koorders 10624, 16005, 16025

\section{POLYALTHIA}

Polyalthia celebica Miq.

Sulawesi distribution - North, Central, South Altitude $-750 \mathrm{~m}$

Collection(s) - bb 5556; Burley, Partomihardjo \& Rachman 3526; Burley et al. 3821, 3881, 3941, 4124, 4273; De Vogel \& Vermeulen 7016; Johansson 167; Koorders 15995, 16018, 16022, 16037, 16038; Lam 2820; Meijer 10761, 10994; Milliken 890; Riedel 5819; Whitmore \& Sidiyasa TCW 3412

Polyalthia glauca Boerl.

Sulawesi distribution - North, South-East 
Altitude $-750 \mathrm{~m}$

Collection(s) - bb 6646, 21324; Bogor Botanical Garden X.F 5, X.F 5a, XX.D 37; Burley et al. 3859; Koorders 15983, 15993, 16006, 16020

\section{Polyalthia lateriflora King}

Sulawesi distribution - South-East

Altitude $-200 \mathrm{~m}$

Collection(s) - Prawiroatmodjo \& Maskuri 1497

Polyalthia lateriflora (Blume) King var. elongata Boerl.

Sulawesi distribution - North

Altitude - 0-1000 m

Collection(s) - De Vogel 2487, 2592; Koorders 15985,17523

\section{Polyalthia rumphii Merr.}

Sulawesi distribution - North, South

Altitude $-800 \mathrm{~m}$

Collection(s) - Burley, Partomihardjo \& Rachman 3577; Burley et al. 3600, 3838, 3869; Cel II 482; Darnaedi 2301; De Vogel \& Vermeulen 6676; Koorders 15980, 15988, 15990 , $15992,16021,16034,16035$

\section{Polyalthia spec.}

Sulawesi distribution - North, Central, South, South-East

Altitude $-750 \mathrm{~m}$

Collection(s) - bb 15084, 17503, 17970, 19607 , 21390, 21765, 24968, 31851, 31883; Burley, Partomihardjo \& Rachman 4218; Burley et al. 3836, 3922, NGS 4053; Cel II 446, II 491, V191; De Vogel 6061; De Vogel \& Vermeulen 6553, 6672, 6905; Forman 231; Koorders 16031; Prawiroatmodjo \& Soewoko 1774

\section{POPOWIA}

\section{Popowia pisocarpa Endl.}

Sulawesi distribution - North, Central

Altitude - 300-700 m

Collection(s) - De Vogel \& Vermeulen 6948; Ramlanto \& Fanani 618

Popowia spec.

Sulawesi distribution - North, South
Altitude $-1000 \mathrm{~m}$

Collection(s) - bb 17683; Koorders 16026, 16028; Meijer 10782; Van Balgooy 3963; Whitmore \& Sidiyasa TCW 3507

\section{PSEUDUVARIA}

\section{Pseuduvaria spec.}

Sulawesi distribution - North

Altitude - 160-750 m

Collection(s) - De Vogel 2571; De Vogel \& Vermeulen $6639,6794,6970$

\section{SAGERAEA}

\section{Sageraea lanceolata Miq.}

Sulawesi distribution - North

Altitude $-150 \mathrm{~m}$

Collection(s) - Burley, Partomihardjo \& Rachman 4046

\section{UVARIA}

Uvaria grandiflora Roxb. ex Hornem.

Sulawesi distribution - North, South, SouthEast

Altitude - 20-400 m

Collection(s) - De Vogel 2528, 5780; Prawiroatmodjo \& Maskuri 1387, 1459; Soejatmi 263

Uvaria littoralis (Blume) Blume

Sulawesi distribution - South-East

Altitude $-80 \mathrm{~m}$

Collection(s) - Amir 162

\section{Uvaria spec.}

Sulawesi distribution - Central, South-East Altitude $-250 \mathrm{~m}$

Collection(s) - Coode 6213; Elbert 3312, 3321; Prawiroatmodjo \& Maskuri 1456; Prawiroatmodjo \& Soewoko 1882, 1910; Sidiyasa \& Didi 1829

\section{XYLOPIA}

\section{Xylopia malayana Hook.f. \& Thomson}

Sulawesi distribution - North, Central, South, South-East

Altitude $-10-200 \mathrm{~m}$ 
Collection(s) - bb 21349, 29474; Cel II 493; Church \& Ismail 19

\section{Xylopia spec.}

Sulawesi distribution - North, South Altitude $-400 \mathrm{~m}$

Collection(s) - bb 22949, 31874; Cel II 266, 472; De Vogel et al. 6183, 6191

\section{APOCYNACEAE}

\section{ALSTONIA}

\section{Alstonia pneumatophora Backer ex Den Berger}

Vernacular name(s) - Lingaroe épé

Sulawesi distribution - South

Altitude $-50 \mathrm{~m}$

Collection(s) - bb 32360, 32601; Cel III 51; Sidiyasa 1381

\section{Alstonia scholaris R.Br.}

Vernacular name(s) - Lingaroekoeni; Pampurud'a; Rita; Sepate, Ampanga [Bugis, Palopo]

Sulawesi distribution - North, Central, South, South-East

Altitude $-500 \mathrm{~m}$

Collection(s) - bb 21595, 24104, 24996; Cel I 63, V 141; De Vriese \& Teijsmann 46; Koorders 15803, 16043, 16078, 19735; Lam 2578; Meijer 9276, 10703; Sidiyasa 1100, 1337, 1386, 1399, 1402, 1409; Teijsmann HB 12516

\section{Alstonia spectabilis R. Br.}

Vernacular name(s) - Hoi; Kayu Tandu; Labuangi [Kalili]; Saoengponisi

Sulawesi distribution - North, Central, South, South-East

Altitude - $2050 \mathrm{~m}$

Collection(s) - bb 3685, 5849, 21603, 22950, 22984, 25159, 26272; Cel I 44, II 210, II 458; De Vogel 5717, 5838, 6144, 6232, 6285, 6313; Koorders 16041, 16042, 16044, 19745, 24068; McDonald \& Ismail Cat 3857; Meijer 11007, 11225; Prawiroatmodjo \& Soewoko 1735, 1836; Rachmat 846; Ramlanto 206; Sidiyasa 1101, 1311, 1384, 1398; Sidiyasa \& Leeuwenberg 1348, 1387; Van Balgooy 3693,3997

\section{Alstonia spec.}

Sulawesi distribution - South-East

Altitude - $150 \mathrm{~m}$

Collection(s) - Prawiroatmodjo \& Maskuri 1177

\section{ALYXIA}

Alyxia celebica D.J. Middleton

Sulawesi distribution - South

Altitude - $1900 \mathrm{~m}$

Collection(s) - Sands 468; Teijsmann HB 14010

Alyxia kabaenae Markgr.

Sulawesi distribution - South-East

Altitude $-200 \mathrm{~m}$

Collection(s) - Elbert 3415

\section{CERBERA}

Cerbera floribunda K. Schum.

Sulawesi distribution - South-East

Altitude - 20-250 m

Collection(s) - Prawiroatmodjo \& Soewoko 1819

\section{Cerbera manghas L.}

Vernacular name(s) - Mangga beraboe

Sulawesi distribution - North, Central, South, South-East

Altitude - 0-320 m

Collection(s) - Elbert 2723, 2803; Eyma 3722; Koorders 16051; Lam 2486; Meijer 10049; Noerkas 226; Rachmat 178

Cerbera odollam Gaertn.

Sulawesi distribution - South, South-East Altitude $-10 \mathrm{~m}$

Collection(s) — bb 20760; Rachmat 148

Cerbera spec.

Sulawesi distribution - North, South-East

Altitude - 0-750 m

Collection(s) - Elbert 2991, 3334; Milliken 1144 


\section{CHILOCARPUS}

Chilocarpus denudatus Blume

Sulawesi distribution - Central

Collection(s) - Ramlanto \& Fanani 661

ICHNOCARPUS

Ichnocarpus frutescens (L.) W.T. Aiton

Sulawesi distribution - North, Central

Altitude - 220-650 m

Collection(s) - Coode 5865; De Vogel \& Vermeulen 6658; Johansson 377; Kaudern 111

Ichnocarpus rhombifolius (Markgr.)

D.J. Middleton

Sulawesi distribution - Central

Lore Lindu National Park \& environs - present

Altitude $-1000 \mathrm{~m}$

Collection(s) - De Vogel 5551

Ichnocarpus serpyllifolius (Blume) P.I. Forst.

Sulawesi distribution - North, Central, South, South-East

Altitude - 20-1100 m

Collection(s) - Bloembergen 4235; De Joncheere 1154; De Vogel 2589; De Vogel \& Vermeulen 6969; Forsten 13, 80; Prawiroatmodjo \& Soewoko 1716

\section{Ichnocarpus spec.}

Sulawesi distribution - North

Altitude - $1000 \mathrm{~m}$

Collection(s) - De Vogel \& Vermeulen 7102

\section{KIBATALIA}

Kibatalia arborea (Blume) G. Don

Vernacular name(s) - Soliti

Sulawesi distribution - South, South-East

Altitude $-15-30 \mathrm{~m}$

Collection(s) - bb 5871, 21722; Cel IV 150

Kibatalia wigmanii (Koord.) Merr.

Vernacular name(s) - Gora-gora

Sulawesi distribution - North

Altitude $-0-500 \mathrm{~m}$

Collection(s) - bb 18417; Church \& Ismail 1; De Vogel 2560; Koorders 16045, 16046,
$16047,16048,16049,16067$; Whitmore \& Sidiyasa TCW 3471

KOPSIA

\section{Kopsia spec.}

Sulawesi distribution - South

Collection(s) - Cel I 50

\section{LEPINIOPSIS}

\section{Lepiniopsis ternatensis Valeton}

Vernacular name(s) - Samparoai

Sulawesi distribution - North, Central, South Altitude - 100-750 m

Collection(s) - Cel II 466; De Vogel 5637, 6114, 6175; Kjellberg 2085; Lam 2615, 2882, 3101; Meijer 10076, 10150, 10725; Teijsmann HB 12439; Van Balgooy 3577

\section{MELODINUS}

\section{Melodinus orientalis Blume}

Sulawesi distribution - North

Collection(s) - Koorders 16073; Van Balgooy 3411

\section{Melodinus spec.}

Sulawesi distribution - South

Collection(s) - bb 1877; De Vogel 5495

\section{PARSONSIA}

Parsonsia alboflavescens (Dennst.) Mabb.

Vernacular name(s) - Mamintax'a

Sulawesi distribution - North, South, SouthEast

Altitude $-0-250 \mathrm{~m}$

Collection(s) - Elbert 2562; Forsten 317; Koorders 16079, 16084, 16085, 16222, 16227; Lam 3340; Prawiroatmodjo \& Soewoko 1804; Van Vuuren 155, 187

\section{Parsonsia celebica (Oliv.) Sleesen}

Sulawesi distribution - North

Collection(s) - Koorders, 16070, 16071, 16075, 19524 


\section{RAUVOLFIA}

\section{Rauvolfia kamarora Hendrian}

Sulawesi distribution - Central

Lore Lindu National Park \& environs - present

Altitude $-700 \mathrm{~m}$

Collection(s) - Hendrian HN 42

\section{Rauvolfia sumatrana Jack}

Vernacular name(s) - Ba'aròsa; Kayu telor (= egg-wood)

Sulawesi distribution - North, South, SouthEast

Altitude - 0-900 m

Collection(s) - bb 17155, 19695; De Vriese 13; De Vriese \& Teijsmann 22; Elbert 2647, 2758; Forman 304; Forsten 86; Koorders 16054, 16056, 16057, 16053; Lam 3124; Prawiroatmodjo \& Maskuri 1499

Rauvolfia verticillata (Lour.) Baill.

Sulawesi distribution - North, Central

Altitude - 650-1000 m

Collection(s) - Bloembergen 4196; Johansson 559; Van Balgooy 3082

\section{STROPHANTHUS}

Strophanthus caudatus (L.) Kurz

Sulawesi distribution - Central, South, SouthEast

Altitude - 0-320 m

Collection(s) - Amir 154; Elbert 2648, 2736, 6235, 6236; Eyma 4195; Kaudern 468; Noerkas 303; Prawiroatmodjo \& Soewoko 1833

\section{Strophanthus spec.}

Sulawesi distribution - South, South-East

Altitude - 200-400 m

Collection(s) — De Vogel 5786

\section{TABERNAEMONTANA}

Tabernaemontana orientalis $\mathrm{R}$. Br.

Sulawesi distribution - Central

Lore Lindu National Park \& environs - present

Altitude - 5-25 m

Collection(s) - bb 21; Eyma 1752
Tabernaemontana pandacaqui Lam.

Sulawesi distribution - North, Central, South, South-East

Altitude $-0-320 \mathrm{~m}$

Collection(s) - Alston 16092; De Vogel 5015; Elbert 2661, 2661a, 2708, 3296; Forsten 84; Kaudern 479; Koorders 15807, 15809, 15812, 15813, 15815, 15818, 16065; Meijer 9179; Riedel HB 5827; Yusuf \& Wahyono 33

\section{VOACANGA}

Voacanga grandifolia (Miq.) Rolfe

Sulawesi distribution - North

Altitude - 0-600 m

Collection(s) - Kaudern 58; Koorders 16059; Riedel 5713; Teijsmann HB 5713

\section{WRIGHTIA}

Wrightia pubescens $\mathrm{R} . \mathrm{Br}$.

Sulawesi distribution - North, Central

Altitude - 150-600 m

Collection(s) - Alston 16098; Meijer 9273, 10226; Van Balgooy 3622

Wrightia pubescens R. Br. subsp. pubescens

Vernacular name(s) - Doedji; Wolato

Sulawesi distribution - North, Central, SouthEast

Altitude - 15-350 m

Collection(s) - bb 15045, 21611, 24979; Teijsmann HB 13602; Van Vuuren 251

\section{AQUIFOLIACEAE}

\section{ILEX}

\section{Ilex cymosa Blume}

Sulawesi distribution - South, South-East Altitude - 15-1100 m

Collection(s) - bb 21276, 33071; Meijer 11179, 11448; Prawiroatmodjo \& Soewoko 1638

\section{Ilex spec.}

Vernacular name(s) - Soeloei

Sulawesi distribution - North, Central, South Lore Lindu National Park \& environs - present Altitude - 350-2450 m 
Collection(s) - bb 28245; Cel III 9; Darnaedi 2103; De Vogel 5375, 5636, 6167; Eyma 4016; Van Balgooy 3325, 3727, 3787, 3982

\section{ARALIACEAE}

\section{OSMOXYLON}

Osmoxylon masarangense Philipson

Sulawesi distribution - North

Altitude - $1200 \mathrm{~m}$

Collection(s) - Forman 209

\section{SCHEFFLERA}

Schefflera elliptica Harms var. microphylla F.M. Mull.

Sulawesi distribution - South

Altitude $-600 \mathrm{~m}$

Collection(s) - Docters van Leeuwen 1728

Schefflera nodosa F.M. Mull.

Sulawesi distribution - North, Central, South Lore Lindu National Park \& environs - present Altitude - 0-1000 m

Collection(s) - De Vogel 5175; Teijsmann HB 5292, HB 5305; Van Balgooy 3083, 3575, 4071

\section{Schefflera polybotrya Koord.}

Sulawesi distribution - South

Collection(s) - Afriastini 2133

Schefflera serrata (Miq.) R. Vig.

Sulawesi distribution - North

Collection(s) - Alston 16244; Koorders 16107

\section{Schefflera spec.}

Vernacular name(s) - Marokaliki/Tambibile Sulawesi distribution - North, Central, South, South-East

Altitude - 20-2200 m

Collection(s) - Bünnemeyer 11833; Cel V 215; Chin 3607; Coode 5904, 6179; Darnaedi 1605; De Vogel 5295, 5350, 5399, 5452, 5467, 5956; De Vogel \& Vermeulen 7069, 7180, 7194; Prawiroatmodjo \& Soewoko 2004; Sands 345, 399, 560; Teijsmann HB 11906, HB 12383; Van Balgooy 3231, 3274, 3280, 3403, 3440; Yusuf \& Wahyono 73

\section{ARAUCARIACEAE}

\section{AGATHIS}

Agathis celebica (Koord.) Warb.

Vernacular name(s) - Damar kadjoe lotang; Damar kapas; Damar mas; Damar sawah(?); Damar wana; Damara; Kawo kawo; Koelontoeoe

Sulawesi distribution - North, Central, South Altitude $-1800 \mathrm{~m}$

Collection(s) - bb 17379, 17380, 17593, 17674, $17675,17676,19688,20016,20330,20546$, $20547,20548,20788,20789,20790,20792$, 20793, 20794, 20795, 20796, 20797, 20798, 20799, 20800, 20801, 20802, 20803, 20804, 20805, 20818, 20819, 20820, 20821, 20822, 20874, 20875, 20876, 20878, 20879, 20880, $20885,20886,21298,21572,21572 a, 21573$, $21582,21641,21642,21646,21647,21648$, $23575,23576,23841,23842,23843,23844$, $23898,24094,24095,26644,27054,28308$, $28397,30153,30165,30166,30171,31501$, $31503,31504,31505,31507,31510,31511$, 31898; Boschproefstation 345; Koorders 15847,15849

\section{Agathis dammara Rich.}

Vernacular name(s) - Damar; Saloe ranté; Solo; Toga

Sulawesi distribution - Central, South

Lore Lindu National Park \& environs - present Altitude $-2200 \mathrm{~m}$

Collection(s) - bb 13632, 15157, 15159, 17562 , 17972, 17973, 18119, 18120, 20275, 20281, 20808, 20809, 20810, 20811, 20812, 20813, 20814, 20815, 20816, 21293, 21580, 21581, $22007,23357,23358,23653,24155,24202$, $24203,24953,28148,28152,28275,28288$, 29002, 29010, 29015, 29189, 29190, 30146, $30147,30154,30155,30156,30157,30158$, $30159,30160,30161,30162,30163,30164$, $31155,31156,31456,31457,31497,31498$, 31499, 31906; Eyma 1316; Rachmat 664; Tantra 1568,1600

\section{Agathis spec.}

Sulawesi distribution - Central Lore Lindu National Park \& environs - present Altitude - $2000 \mathrm{~m}$

Collection(s) - De Vogel 5423 


\section{ASCLEPIADACEAE \\ CALOTROPIS}

Calotropis gigantea (L.) Dryand. ex W.T. Aiton

Sulawesi distribution - North

Collection(s) - Afriastini \& Rohajawati JJA 2952

\section{AVICENNIACEAE}

\section{AVICENNIA}

\section{Avicennia alba Blume}

Sulawesi distribution - North, South

Altitude $-2 \mathrm{~m}$

Collection(s) - bb 14440; Teijsmann HB 13766; Van Vuuren 357

Avicennia alba Blume var. latifolia Mold.

Sulawesi distribution - South

Collection(s) - Noerkas 58

Avicennia marina (Forssk.) Vierh.

Vernacular name(s) - Api-api; Wapi mopoetio

Sulawesi distribution - North

Altitude - 0-2 m

Collection(s) - bb 14441, 15570; Meijer 9289; Van Vuuren 397

Avicennia marina (Forssk.) Vierh. var. resinifera (G. Forst.) Bakh.

Sulawesi distribution - South

Collection(s) - Teijsmann HB 13791

Avicennia marina (Forssk.) Vierh. var. rumphiana (Hallier f.) Bakh.

Vernacular name(s) - Api-api; Api/Piapi; Dalita; Molontejapoe

Sulawesi distribution - North, South, SouthEast

Altitude $-0-3 \mathrm{~m}$

Collection(s) - bb 3637, 16715, 17196, 19698, 21795; Lam 2675

\section{Avicennia officinalis L.}

Vernacular name(s) - Api-api

Sulawesi distribution - South, South-East

Altitude $-0-100 \mathrm{~m}$
Collection(s) - bb 3891; Docters van LeeuwenReynvaan 1934; Elbert 2701; Teijsmann HB 13767

\section{BIGNONIACEAE}

\author{
DEPLANCHEA
}

Deplanchea bancana (Scheff.) Steenis

Vernacular name(s) - Kalamboetoh [Toradjasih]

Sulawesi distribution - South

Altitude - 100-500 m

Collection(s) - bb 24135; Cel III 1; Van Balgooy $3798,3810,3833,4041$

\section{DOLICHANDRONE}

Dolichandrone spathacea (L.f.) K. Schum.

Vernacular name(s) - Djaran

Sulawesi distribution - North, South, SouthEast

Altitude - 1-25 m

Collection(s) - bb 3683, 20049, 22073; Koorders 16250, 16252; Noerkas 55

\section{MILLINGTONIA}

\section{Millingtonia hortensis L.f.}

Vernacular name(s) - Kaoelolo

Sulawesi distribution - South, South-East

Altitude $-12-25 \mathrm{~m}$

Collection(s) - bb 4998, 22066; Noerkas 332

\section{OROXYLUM}

\section{Oroxylum indicum (L.) Kurz}

Sulawesi distribution - Central, South, SouthEast

Altitude - 5-310 m

Collection(s) - bb 21357, 24111; Bünnemeyer 10931; Cel V 139; Meijer 10813; Noerkas 419; Ramlanto 239

\section{PANDOREA}

Pandorea pandorana (Andr.) Steenis

Sulawesi distribution - South

Altitude $-500 \mathrm{~m}$

Collection(s) _- Van Balgooy 3915 


\section{RADEMACHERA}

Radermachera pinnata (Blanco) Seem.

Vernacular name(s) - Risih; Tive, kaoe

Sulawesi distribution - North, South, SouthEast

Altitude - 0-900 m

Collection(s) - bb 3925, 19437, 21125, 22991, 25552; Burley, Partomihardjo \& Rachman 3727; Cel III 49; De Vogel 5785; Eyma 1134; Koorders 16253, 16254, 16255, 16256; Meijer 11228

\section{BOMBACACEAE}

BOMBAX

Bombax ceiba L. var. leiocarpum Robyns

Vernacular name(s) - Leleh

Sulawesi distribution - South-East

Altitude $-10 \mathrm{~m}$

Collection(s) - bb 6023, 21355

\section{CAMPTOSTEMON}

Camptostemon philippinensis (Vidal) Becc.

Vernacular name(s) - Kapa; Molinggapoto

Sulawesi distribution - North

Altitude $-0-2 \mathrm{~m}$

Collection(s) - bb 14450, 16713

\section{ERIODENDRON}

Eriodendron anfractuosum DC.

Vernacular name(s) - Kaoe Kaoe

Sulawesi distribution - South

Altitude $-900 \mathrm{~m}$

Collection(s) - Bünnemeyer 12568

\section{BORAGINACEAE}

CORDIA

\section{Cordia spec.}

Sulawesi distribution - North, Central, SouthEast

Altitude $-0-500 \mathrm{~m}$

Collection(s) - Burley, Partomihardjo \& Rachman 3557; Coode 5911, 6089; De Vogel \& Vermeulen $6455,6661,6987$

\section{HELIOTROPIUM}

Heliotropium indicum L.

Sulawesi distribution - South

Collection(s) - Chin 3565

\section{TOURNEFORTIA}

Tournefortia sarmentosa Lam.

Sulawesi distribution - North, South

Altitude - 220-400 m

Collection(s) - De Vogel 5946; De Vogel \& Vermeulen 6994

\section{BUDDLEJACEAE}

\section{BUDDLEJA}

Buddleja asiatica Lour.

Sulawesi distribution - North, Central, South Lore Lindu National Park \& environs - present Altitude - 500-1650 m

Collection(s) - Alston 15899; Bloembergen 4150; Bünnemeyer 12106; Eyma 1688; Forsten 57; Johansson 412; Koorders 17731, 17732, 17733; Sands 509

\section{BURSERACEAE}

\section{CANARIUM}

Canarium acutifolium (DC.) Merr. var. celebicum Leenh.

Sulawesi distribution - North, Central, South, South-East

Altitude - 20-400 m

Collection(s) - bb 28279; Cel V 190, V 310; Prawiroatmodjo \& Soewoko 1680

\section{Canarium asperum Benth.}

Vernacular name(s) - Biolo; damar-damar; Usaree

Sulawesi distribution - North, Central, South, South-East

Altitude $-3-400 \mathrm{~m}$

Collection(s) - bb 5428, 15128, 17504, 21109 , 21308, 31911; De Vogel 2567; Elbert 3443; Koorders 16298, 16300, 16317; Lam 2580; Meijer 9239, 10127; Noerkas 377 
Canarium asperum Benth. forma villosum (Blume) H.J. Lam

Sulawesi distribution - North, Central, South, South-East

Altitude $-3-800 \mathrm{~m}$

Collection(s) - bb 6650, 12656, 17047, 24706, 31866; Koorders 16305, 16310, 16315, 16318; Lam 2437; Rachmat 685; Teijsmann HB 12225

\section{Canarium balsamiferum Willd.}

Vernacular name(s) - Karematu [Tolaki]; Matimpoene; Mularan

Sulawesi distribution - North, Central, South, South-East

Altitude - 0-1000 m

Collection(s) - bb 7532, 17566, 28218, 28235; Cel 234, II 129, II 478; Coode 6077; De Vogel 6214; Koorders 16296; Lam 2870; Meijer 10914; Posthumus 2344; Teijsmann HB 11974, HB 12384, HB 12424; Van Balgooy 3116,4054

\section{Canarium hirsutum Willd.}

Vernacular name(s) - Kau Tangali [Besoa]; Nawowang [Tambulu]; Raganogaloe; Tontong

Sulawesi distribution - North, Central, South, South-East

Altitude - 0-1150 m

Collection(s) - bb 5431, 17289, 19603, 21389; Burley, Partomihardjo \& Rachman 3751; Cel I 1, I 94, V 282; De Vogel 2438, 5269, 5585, 5632; Keßler et al. PK 3174; Koorders 16293, 16295, 16306, 16309, 16312, 16401; Meijer 10711; Prawiroatmodjo \& Soewoko 1871; Teijsmann HB 14170

Canarium maluense Lauterb.

Sulawesi distribution - South

Altitude $-780 \mathrm{~m}$

Collection(s) - bb 26015

Canarium maluense Lauterb. subsp. celebicum Leenh.

Vernacular name(s) - Malapau [South lan]

Sulawesi distribution - South

Altitude - 50-420 m

Collection(s) - bb 13573; Cel II 413; De Vogel 6096, 6131; Meijer 11250
Canarium maluense Lauterb. subsp. maluense

Sulawesi distribution - South

Altitude $-400 \mathrm{~m}$

Collection(s) - De Vogel 6220

Canarium trigonum Lam.

Sulawesi distribution - South

Altitude $-200 \mathrm{~m}$

Collection(s) - Cel II 401

Canarium vrieseanum Engl. forma vrieseanum

Vernacular name(s) - Kan ne Oewal

Sulawesi distribution - North

Altitude $-200-340 \mathrm{~m}$

Collection(s) - bb 14151, 19414; De Vogel \& Vermeulen 6601, 6669, 6722, 6912; Hochreutiner 131; Koorders 16302, 16313, 16314; Whitmore \& Sidiyasa TCW 3470, TCW 3550

\section{Canarium vulgare Leenh.}

Vernacular name(s) - Kanari

Sulawesi distribution - North, South

Altitude - 50-260

Collection(s) - Alston 16169; bb 15050, 24114; Forsten 438; Lam 3131; Raringpandey 2

\section{Canarium spec.}

Sulawesi distribution - North, Central

Altitude - 50-1040 m

Collection(s) - De Vogel 2656; Keßler et al. PK 3173

\section{DACRYODES}

Dacryodes rostrata (Blume) H.J. Lam forma cuspidata (Blume) H.J. Lam

Sulawesi distribution - North

Altitude - 0-600 m

Collection(s) - Koorders 16303, 16308

Dacryodes spec.

Sulawesi distribution - Central

Lore Lindu National Park \& environs - present

Altitude - $1040 \mathrm{~m}$

Collection(s) - Keßler et al. PK 3162 


\section{GARUGA}

Garuga floribunda Decne.

Vernacular name(s) - Adjoe barroe; Bogoe; Kajoe kambing; Kambing; Wooe

Sulawesi distribution - North, Central, South, South-East

Altitude - 5-500 m

Collection(s) - bb 5574, 6036, 15047, 20250, 21336, 28739, 31482; Cel I 30; Elbert 2042; Kamoeliaan 6, 14; Koorders 16326, 16327; Lam 2473, 3178; Politon 90; Teijsmann HB 11819, HB 12277, HB 12498, HB 13868

\section{HAPLOLOBUS}

Haplolobus floribundus H.J. Lam subsp. moluccanus Leenh.

Sulawesi distribution - Central, South, SouthEast

Altitude - 20-500 m

Collection(s) - bb 208, 26279, 31850, 31901; Cel V 312; Meijer 11258; Prawiroatmodjo \& Soewoko 1700, 1927, 1940; Van Balgooy 3860

\section{SANTIRIA}

\section{Santiria apiculata Benn.}

Sulawesi distribution - South

Altitude $-25 \mathrm{~m}$

Collection(s) - bb 24503

Santiria brevisepala Leenh.

Vernacular name(s) - Kobili

Sulawesi distribution - South-East

Altitude - 60-200 m

Collection(s) - bb 32329; Rantegaoe 25

\section{Santiria laevigata Blume}

Vernacular name(s) - Kala-kala Babi; Tapi-tapi Sulawesi distribution - North, Central, South, South-East

Altitude - 30-500 m

Collection(s) - bb 1870, 8564, 23252, 31824, 31878; Cel II 216, II 238; Ramlanto \& Fanani 735; Reppie 11, 30, 39

\section{CAPPARACEAE}

\section{CAPPARIS}

\section{Capparis cantoniensis Lour.}

Sulawesi distribution - South, South-East

Altitude $-0-250 \mathrm{~m}$

Collection(s) - Forsten 344; Kostermans \& Soegeng Reksodihardjo 7; Prawiroatmodjo \& Soewoko 1722

Capparis gynandra (L.) Briq.

Vernacular name(s) - Tjamangih

Sulawesi distribution - North, Central, South Lore Lindu National Park \& environs - present Altitude $-300 \mathrm{~m}$

Collection(s) - Kaudern 199; Koorders 16347; Meijer 10157; Noerkas 20; Rachmat 239

\section{Capparis lanceolaris DC.}

Sulawesi distribution - North, South, SouthEast

Altitude - 0-320 m

Collection(s) - Elbert 6436; K.F. Sarasin \& P.B. Sarasin 784; Teijsmann HB 12124, HB 13835

Capparis lucida (Banks ex DC.) Benth.

Sulawesi distribution - South-East

Altitude - 25-150 m

Collection(s) - Elbert 2984

Capparis micracantha DC.

Vernacular name(s) - Kajooesompa

Sulawesi distribution - North, Central, South, South-East

Altitude $-0-300 \mathrm{~m}$

Collection(s) - bb 14; De Vogel 5027; Elbert 2983, 9271; Forsten 324; Kaudern 402; Koorders 16339; Meijer 9211; Noerkas 172; Prawiroatmodjo \& Soewoko 1754, 1948; Teijsmann HB 12092

\section{Capparis pubiflora DC.}

Sulawesi distribution - North, Central, South, South-East

Altitude $-0-2000 \mathrm{~m}$

Collection(s) - Coode 5837; De Joncheere 1069; De Vogel 5084; Elbert 2981; Forsten 392; Johansson 114, 388; Koorders 16336 , 
16337, 16340, 16341, 16351, 16352; Tantra 1521; Teijsmann HB 12126, HB 12274, HB 12537; Van Balgooy 3024, 3034

Capparis quiniflora DC.

Sulawesi distribution - Central

Lore Lindu National Park \& environs - present

Altitude - 100-200 m

Collection(s) - Meijer 10154

Capparis zeylanica $L$.

Sulawesi distribution - Central, South

Lore Lindu National Park \& environs - present

Altitude - 100-200 m

Collection(s) - Meijer 10156, 10171; Van Vuuren 91,173

\section{Capparis spec.}

Sulawesi distribution - North

Altitude $-270 \mathrm{~m}$

Collection(s) - Whitmore \& Sidiyasa TCW 3530

\section{CLEOME}

\section{Cleome viscosa $\mathrm{L}$.}

Vernacular name(s) - Ahuru; Joempai; Toelang toradja

Sulawesi distribution - North, South, SouthEast

Altitude - 0-320 m

Collection(s) - Alston 16371; Elbert 2135, 2528, 6214; Forsten 47; Kartawinata 1273; Koorders 16349; Lam 3438; Noerkas 34; Rachmat 268; Teijsmann HB 12461; Van Vuuren 70

\section{CRATEVA}

Crateva religiosa G. Forst.

Vernacular name(s) - Balalehe; diu; Papangi nasu

Sulawesi distribution - North, South-East

Altitude - 0-200 m

Collection(s) - bb 4993; Elbert 3330; Koorders 16335, 16343, 16344, 16345, 16346, 19565; Lam 3081; Prawiroatmodjo \& Maskuri 1193; Widjaja 601

\section{CAPRIFOLIACEAE}

\section{SAMBUCUS}

Sambucus javanica Reinw. ex Blume

Sulawesi distribution - North, Central, South Altitude - 1000-2000 m

Collection(s) - Bünnemeyer 11762; Koorders 16355; Meijer 9980; Rachmat 428; Teijsmann HB 13945; Van Balgooy 3183, 3522

\section{VIBURNUM}

\section{Viburnum amplificatum Kern}

Sulawesi distribution - Central

Lore Lindu National Park \& environs - present Altitude - 1700-1750 m

Collection(s) - De Vogel 5448; Van Balgooy 3406

\section{Viburnum odoratissimum Ker-Gawl.}

Sulawesi distribution - North, South

Altitude - 6-2500 m

Collection(s) - Alston 15862; bb 18101; Eyma 997; Koorders 16356, 16361, 16362, 16363, 16364,16365

Viburnum sambucinum Reinw.

Sulawesi distribution - North, Central, South, South-East

Altitude - 600-1200 m

Collection(s) - Bloembergen 4081, 4095; Bünnemeyer 11647, 11700; Elbert 3455, 3488; Eyma 3762; Meijer 9724; Rachmat 1020; Teijsmann HB 13982; Van Balgooy 3140

\section{Viburnum spec.}

Sulawesi distribution - Central, South Lore Lindu National Park \& environs - present Altitude $-1060 \mathrm{~m}$

Collection(s) - Keßler et al. PK 3138; Yoshida 1349,1393 


\section{CASUARINACEAE}

\section{CASUARINA}

Casuarina equisetifolia L. ex J.R. Forst. \& G. Forst. subsp. equisetifolia

Sulawesi distribution - South-East

Altitude $-0-10 \mathrm{~m}$

Collection(s) - Elbert 2526; Teijsmann HB 13890

Casuarina oligodon L.A.S. Johnson subsp. celebica L.A.S. Johnson

Sulawesi distribution - Central, South

Altitude - 1-850 m

Collection(s) - bb 19822, 23902; Eyma 3525, 3730

\section{GYMNOSTOMA}

Gymnostoma rumphianum (Miq.) L.A.S. Johnson

Vernacular name(s) - Wahoe

Sulawesi distribution - North, Central, South

Altitude $-30-800 \mathrm{~m}$

Collection(s) - bb 15599, 16653, 28242; Bloembergen 4262; Eyma 3816; Koorders 16366, $16367,16369,16371$; Noerkas 329; Posthumus 2663

Gymnostoma sumatranum (Jungh. ex de Vriese) L.A.S. Johnson

Vernacular name(s) - Ihaoe oenroe / Adjoe; Uangin [Tator]

Sulawesi distribution - North, Central, South, South-East

Altitude - 0-1000 m

Collection(s) - bb 1828, 21589, 31908, 32463, 32600; Cel II 237, II 276, II 378; Coode 5880, 5882; De Vogel 6166; Elbert 3119, 3212, 3489; Kaudern 185; Van Balgooy 3690

\section{CECROPIACEAE}

POIKILOSPERMUM

Poikilospermum suaveolens (Blume) Merr.

Vernacular name(s) - Tomboe

Sulawesi distribution - North, Central, South, South-East
Altitude - 0-1200 m

Collection(s) - Bloembergen 4198; Elbert 3373; Eyma 1156; Meijer 9696, 9956, 10777 , 10799; Posthumus 2496; Ramlanto \& Fanani 510

\section{Poikilospermum spec.}

Vernacular name(s) - harotonga; tongulu [Soroako/Kendari]; Tombu [Besoa]

Sulawesi distribution - North, Central, South, South-East

Altitude - 20-1000 m

Collection(s) - De Vogel 5538; Hennipman 5056, 5768, 5971; Prawiroatmodjo \& Soewoko 1785; Whitmore \& Sidiyasa 3430

\section{CELASTRACEAE}

\section{ARNICRATEA}

Arnicratea grahami (Wight) Ding Hou

Sulawesi distribution - South

Altitude $-400 \mathrm{~m}$

Collection(s) - De Vogel 5794

\section{CELASTRUS}

\section{Celastrus hindsii Benth.}

Sulawesi distribution - South-East

Altitude - 200-300 m

Collection(s) - Prawiroatmodjo \& Soewoko 1946

\section{Celastrus monospermoides Loes.}

Sulawesi distribution - South

Altitude - $1900 \mathrm{~m}$

Collection(s) - Sands 469

\section{Celastrus paniculatus Willd.}

Sulawesi distribution - North, South

Collection(s) - Koorders 19632; Lam 2466; Teijsmann HB 13577

\section{EUONYMUS}

Euonymus acuminifolius Blakelock

Sulawesi distribution - North, South

Lore Lindu National Park \& environs — present Altitude - 1000-3200 m 
Collection(s) - Eyma 526, 705; Koorders 16378; Meijer 9691, 9716

\section{Euonymus cochinchinensis Pierre}

Vernacular name(s) - ampah-loleh; boenga sanggara

Sulawesi distribution - Central, South

Altitude - 20-2950 m

Collection(s) - De Vogel 5666; Kofman 239; Sands 208, 250; Van Vuuren 169, 171

Euonymus impressus Blakelock

Sulawesi distribution - Central

Collection(s) - Rachmat 544

Euonymus javanicus Blume

Vernacular name(s) - ligisata' a; sandoe moloba

Sulawesi distribution - North, South, SouthEast

Altitude - 250-1250 m

Collection(s) - bb 14340, 14539; Burley, Partomihardjo \& Rachman 3888, 4014; Cel V 217; Koorders 16373, 16376; Lam 3169; McDonald \& Ismail 3876, 3877, 4020, 4130

\section{Euonymus spec.}

Sulawesi distribution - Central

Lore Lindu National Park \& environs - present

Altitude $-1040 \mathrm{~m}$

Collection(s) - Keßler et al. PK 3172

\section{GLYPTOPETALUM}

\section{Glyptopetalum loheri Merr.}

Sulawesi distribution - South-East

Collection(s) - Kjellberg 499

\section{LOESENERIELLA}

Loeseneriella pauciflora (DC.) A.C. Sm.

Sulawesi distribution - South, South-East

Collection(s) - Kjellberg 46; Teijsmann HB 12264

\section{LOPHOPETALUM}

Lophopetalum javanicum (Zoll.) Turcz.

Vernacular name(s) - Bongkorio; Samoengkoewana
Sulawesi distribution - North, South, SouthEast

Altitude - 0-1500 m

Collection(s) - bb 5863, 19593, 21732, 24075, 29020, 31825; Burley, Partomihardjo \& Rachman 4255; Cel V 145; De Vogel 2454, 6339; Kjellberg 2160; Koorders 19608

\section{Lophopetalum torricellense Loes.}

Sulawesi distribution - South

Altitude $-300 \mathrm{~m}$

Collection(s) - Van Balgooy 3827

Lophopetalum spec.

Sulawesi distribution - North

Collection(s) - Whitmore \& Sidiyasa TCW 3500

\section{PERROTTETIA}

Perrottetia alpestris (Blume) Loes. subsp. alpestris

Sulawesi distribution - South

Collection(s) - Afriastini 2093

Perrottetia alpestris (Blume) Loes. subsp. moluccana (Blume) Ding Hou

Sulawesi distribution - North

Altitude - $400 \mathrm{~m}$

Collection(s) - De Vogel \& Vermeulen 7117

Perrottetia alpestris (Blume) Loes. subsp. philippinensis (Vidal) Ding Hou

Vernacular name(s) - lotong matjaela

Sulawesi distribution - North, South

Altitude - 0-2900 m

Collection(s) - De Vogel \& Vermeulen 7193, 7198; Eyma 727, 1166; Forman 183; Koorders $18180,18181,18182,18185$; Reinwardt 554; Van Vuuren 503, 550; Yoshida 1292; Yusuf \& Wahyono 66

REISSANTIA

Reissantia indica (Willd.) Halle

Sulawesi distribution - South-East

Altitude $-20-250 \mathrm{~m}$

Collection(s) - Prawiroatmodjo \& Soewoko 1878 


\section{SALACIA}

Salacia blepharophora Ding Hou

Sulawesi distribution - South

Altitude $-400 \mathrm{~m}$

Collection(s) - Kjellberg 2810; Van Balgooy 3726

Salacia chinensis L.

Sulawesi distribution - South

Altitude - $400 \mathrm{~m}$

Collection(s) - De Vogel 5926

Salacia erythrocarpa K. Schum.

Sulawesi distribution - South

Altitude - 400-425 m

Collection(s) - De Vogel 6237; Hennipman 5822, 5849; Van Balgooy 3655

Salacia forsteniana Miq.

Sulawesi distribution - South

Altitude $-430 \mathrm{~m}$

Collection(s) - De Vogel 6280

Salacia intermedia Ding Hou

Sulawesi distribution - North, South

Collection(s) - Teijsmann HB 14156

Salacia korthalsiana Miq.

Sulawesi distribution - South

Altitude - 400-500 m

Collection(s) - De Vogel 5858; Meijer 11092; Van Balgooy 3715

\section{Salacia kraemeri (Loes.) Ding Hou}

Sulawesi distribution - South

Collection(s) - Kjellberg 2037

Salacia ledermannii (Loes. ex Harms) Ding Hou

Sulawesi distribution - South-East

Altitude - 0-900 m

Collection(s) - Elbert 2823, 2835, 3303; McDonald \& Ismail 3863

\section{Salacia macrophylla Blume}

Sulawesi distribution - South-East

Altitude - 0-497 m
Collection(s) - Elbert 3141, 3178; Forsten 1888; Prawiroatmodjo \& Maskuri 1466; Prawiroatmodjo \& Soewoko 1630,1759

Salacia ovalis Korth.

Sulawesi distribution - Central

Lore Lindu National Park \& environs - present Altitude $-1000 \mathrm{~m}$

Collection(s) - De Vogel 5224; Van Vuuren 863

\section{SIPHONODON}

Siphonodon celastrineus Griff.

Sulawesi distribution - Central

Collection(s) - De Vogel 5586

\section{CHLORANTHACEAE}

ASCARINA

Ascarina philippinensis C.B. Rob.

Sulawesi distribution - South

Altitude $-2000 \mathrm{~m}$

Collection(s) - Eyma 1354

Chloranthus erectus (Buch.-Ham.) Verdc.

Sulawesi distribution - Central

Lore Lindu National Park \& environs - present

Altitude $-200 \mathrm{~m}$

Collection(s) - Coode 5806; Eyma 3487, 3824

HEDYOSMUM

Hedyosmum orientale Merr. \& Chun

Sulawesi distribution - North, Central, South Lore Lindu National Park \& environs - present Altitude - 1300-2360 m

Collection(s) - Bloembergen 3998; Johansson 503; Meijer 9899; Van Balgooy 3227

\section{SARCANDRA}

\section{Sarcandra glabra (Thunb.) Nakai}

Sulawesi distribution - North, South

Lore Lindu National Park \& environs - present Altitude - $1000 \mathrm{~m}$

Collection(s) - Bloembergen 4013; Teijsmann HB 14048 


\section{CHRYSOBALANACEAE}

\section{ATUNA}

Atuna excelsa Benth.

Sulawesi distribution - North

Collection(s) - Burley et al. 4091

\section{KOSTERMANTHUS}

Kostermanthus heteropetalus (Scort. ex King) Prance

Vernacular name(s) - Tabenoe motea

Sulawesi distribution - South

Altitude - 4-300 m

Collection(s) - bb 1820, 1838; Cel II 102, III 16, III 463

\section{LICANIA}

Licania splendens (Korth.) Prance \& Kosterm. Sulawesi distribution - South

Collection(s) - bb 32504

\section{MARANTHES}

Maranthes corymbosa (Blume) Miq.

Vernacular name(s) -- Bareraga; Kolasa, kolaka; Sampomoeli; Welete

Sulawesi distribution - North, Central, South, South-East

Altitude $-10-500 \mathrm{~m}$

Collection(s) - bb 1818, 1901, 4178, 4983, 20903, 23259, 23288, 24507, 31870, 32453, 32489, 33078; Burley, Partomihardjo \& Rachman 4177; Cel II 445, II 494, III 4, IV 141; Koorders 18547, 18548; Meijer 11221; Prawiroatmodjo \& Soewoko 1662

\section{CLETHRACEAE}

\section{CLETHRA}

Clethra canescens Reinw. \& Blume

Vernacular name(s) - Kajoe parang

Sulawesi distribution - North, Central, South Lore Lindu National Park \& environs - present Altitude - 0-2400 m

Collection(s) - Alston 15701; bb 18096; Bün- nemeyer 11890, 12390; Koorders 17527, 19280, 19611; Rachmat 902, 909; Reinwardt 911; Teijsmann HB 14180; Van Balgooy 3196,3261

Clethra canescens Reinw. ex Blume var. canescens

Sulawesi distribution - South

Collection(s) - Yoshida 1126

Clethra longispicata J.J. Sm.

Sulawesi distribution - Central, South

Altitude - 0-1150 M

Collection(s) - bb 24128; Rachmat 553

\section{COMBRETACEAE}

\section{COMBRETUM}

Combretum acuminatum Roxb.

Sulawesi distribution - South

Collection(s) - Teijsmann HB 11931, HB 12805

\section{Combretum latifolium Blume}

Sulawesi distribution - South, South-East Altitude - 0-200 m

Collection(s) - Elbert 3284; Meijer 10797, 10857; Teijsmann HB 12303, HB 14139

Combretum trifoliatum Vent.

Vernacular name(s) - Potapa

Sulawesi distribution - Central, South

Altitude - $80 \mathrm{~m}$

Collection(s) - Amir 73; Teijsmann HB 12182, HB 12192, HB 12270, HB 12871

\section{LUMNITZERA}

Lumnitzera littorea (Jack) Voigt

Vernacular name(s) - Sarvow

Sulawesi distribution - North

Altitude - $1 \mathrm{~m}$

Collection(s) - Lam 3408

Lumnitzera racemosa Willd.

Vernacular name(s) - Pa rapa

Sulawesi distribution - South

Collection(s) - Kjellberg 2454; Noerkas 398 


\section{QUISQUALIS}

\section{Quisqualis indica L.}

Sulawesi distribution - Central, South, SouthEast

Altitude $-0-75 \mathrm{~m}$

Collection(s) - Elbert 2623; Kjellberg 176; Rachmat 732; Teijsmann HB 12337, HB 12590

\section{TERMINALIA}

Terminalia bellirica (Gaertn.) Roxb.

Sulawesi distribution - South

Altitude $-120 \mathrm{~m}$

Collection(s) - bb 25538

Terminalia catappa $L$.

Vernacular name(s) - Hoeton; Tolite

Sulawesi distribution - North, South, SouthEast

Altitude $-0-200 \mathrm{~m}$

Collection(s) - bb 15606, 21796; Elbert 2572, 3282; Eyma 3731; Forsten 461; Koorders 16389; Rachmat 714, 767

Terminalia citrina (Gaertn.) Roxb. ex Fleming

Vernacular name(s) - Tomboelilato bintalo

Sulawesi distribution - North, Central, SouthEast

Altitude $-10-30 \mathrm{~m}$

Collection(s) - bb 15129, 21378, 21748, 31488

Terminalia copelandi Elmer

Vernacular name(s) - Lanoeang'a

Sulawesi distribution - North, Central

Altitude $-20 \mathrm{~m}$

Collection(s) - Coode 5853; Lam 3035; Meijer 10111

\section{Terminalia kjellbergii Exell}

Sulawesi distribution - South

Altitude $-400 \mathrm{~m}$

Collection(s) - Van Balgooy 3777, 3779, 3805

Terminalia microcarpa Decne.

Vernacular name(s) - Talise

Sulawesi distribution - North, Central, South, South-East

Altitude $-0-1000 \mathrm{~m}$
Collection(s) - bb 24709; Cel I 15; Coode 5850; Elbert 2674; Koorders 16390, 16392, 18219, 19566; Lam 2884; Meijer 10783; Teijsmann HB 13839, HB 13932; Van Balgooy 3518

Terminalia samoensis Rech.

Vernacular name(s) - Talise

Sulawesi distribution - North

Altitude $-7 \mathrm{~m}$

Collection(s) - Lam 3370

Terminalia supitiana Koord.

Vernacular name(s) - Tolihé mongkoeni

Sulawesi distribution - North, Central, South

Altitude - 110-500 m

Collection(s) - bb 24487, 31896; Cel III 3; Koorders 18320; Meijer 11434

\section{Terminalia spec.}

Vernacular name(s) - Kajuntahea [Uma]; Mo'ong'a; Salamate [Besoa]

Sulawesi distribution - North, Central, South, South-East

Altitude - 20-1050 m

Collection(s) - bb 19611; De Vogel 5605, 6127, 6276; Lam 2824, 3167; Van Balgooy 3133

\section{COMPOSITAE}

VERNONIA

Vernonia arborea Buch.-Ham.

Vernacular name(s) - boeboek; Donan [Wasuponda]; Lenjanju [Besoa]; Tambone [Besoa]

Sulawesi distribution - North, Central, South, South-East

Altitude - 15-1780 m

Collection(s) - Afriastini 2041; bb 21723, 22987, 24170, 24502, 28251, 32507; Burley, Partomihardjo \& Rachman 4035; Burley et al. 3962; Cel II 332; De Vogel 5159, 5262, 5281, 5298, 6209; De Vogel \& Vermeulen 7214; Forman 374; McDonald \& Ismail 4056; Meijer 9729, 11072; Tantra 1539; Van Balgooy 3144, 3646, 3992

Vernonia arborea Buch.-Ham. var. celebica (Blume) Koster

Sulawesi distribution - North, Central, South Altitude $-1000-2200 \mathrm{~m}$ 
Collection(s) - Bünnemeyer 11701, 119892; Koorders 16507, 19218; Teijsmann HB 14041

\section{Vernonia arborea Buch.-Ham. var. obovata Moore}

Vernacular name(s) - Rogo-Monggane Sulawesi distribution - North, South-East Altitude - 50-100 m

Collection(s) - bb 5424; Kjellberg 672; Koorders $16495,16504,19220$

\section{CONNARACEAE}

\section{CONNARUS}

\section{Connarus grandis Jack}

Sulawesi distribution - North

Collection(s) - Lam 3005

Connarus monocarpus L. subsp. malayensis Leenh.

Sulawesi distribution - North, South, SouthEast

Altitude - 50-400 m

Collection(s) - Beccari 3080; De Vogel 5759, 5845, 6212; Kjellberg 2393; Koorders 16529, 17582; Ramlanto 171

\section{Connarus spec.}

Sulawesi distribution - South-East

Altitude $-100-200 \mathrm{~m}$

Collection(s) - Prawiroatmodjo \& Soewoko 1912

\section{ELLIPANTHUS}

Ellipanthus tomentosus Kurz var. luzoniensis (Vidal) Leenh.

Sulawesi distribution - South

Altitude - 200-500 m

Collection(s) - bb 19816; Van Balgooy 3950

\section{ROUREA}

Rourea minor (Gaertn.) Leenh.

Sulawesi distribution - South

Collection(s) - Teijsmann HB 11809

\section{CONVOLVULACEAE}

\section{ARGYREIA}

Argyreia celebica Ooststr.

Sulawesi distribution - North

Altitude $-500 \mathrm{~m}$

Collection(s) - Koorders 18214

Argyreia cinerea Ooststr.

Sulawesi distribution - North

Collection(s) - K.F. Sarasin \& P. B. Sarasin 523

\section{ERYCIBE}

Erycibe malaccensis C.B. Clarke

Sulawesi distribution - North, Central, South, South-East

Altitude - 0-645 m

Collection(s) - Burley, Partomihardjo \& Rachman 4166; De Vriese 3437; Elbert 3053, 3359; Forsten 346; Kjellberg 682; Prawiroatmodjo \& Soewoko 1793, 1928; Teijsmann HB 11737, HB 11782, HB 11942, HB 11992, HB 12180, HB 12803, HB 13596, HB 13831; Van Vuuren 159, 383, 815

Erycibe subglabra Scheff. ex Hoogland

Sulawesi distribution - South

Collection(s) - Meijer 11184

Erycibe spec.

Sulawesi distribution - North, South

Altitude - $950 \mathrm{~m}$

Collection(s) - Chin 3592; De Vogel 2436

\section{LEPISTEMON}

Lepistemon binectariferum (Wall.) Kuntze var. eymae Ooststr.

Sulawesi distribution - North

Collection(s) - Eyma 3990

\section{CORNACEAE}

MASTIXIA

Mastixia pentandra Blume

Sulawesi distribution - North 
Altitude $-400 \mathrm{~m}$

Collection(s) - Burley, Partomihardjo \& Rachman 3865

Mastixia pentandra Blume subsp. moluccana K.M. Matthew

Sulawesi distribution - Central

Lore Lindu National Park \& environs - present Altitude - 2000-2050 m

Collection(s) - De Vogel 5409, 5429

Mastixia pentandra Blume subsp. scortechinii (King) K.M. Matthew

Sulawesi distribution - North, Central, South Altitude - 1250-2050 m

Collection(s) — bb 20871, 26275, 28265

\section{CRYPTERONIACEAE}

\section{CRYPTERONIA}

Crypteronia cummingii (Planch.) Endl.

Vernacular name(s) - Koemba-a; Langori lauroe; Lengari; Tomo wamma

Sulawesi distribution - South

Altitude $-1800 \mathrm{~m}$

Collection(s) - bb 9704, 24092; Cel II 304, II 347, II 421; Van Balgooy 3843, 3885

\section{CUNONIACEAE}

\section{CALDCLUVIA}

Caldcluvia celebica (Blume) Hoogland

Sulawesi distribution - North, Central, South Altitude - 0-1650 m

Collection(s) - bb 18097, 28273, 33070; Koorders 18018, 18019, 18020, 18024; Meijer 9986; Van Steenis 10318

\section{WEINMANNIA}

Weinmannia celebica Koord.

Sulawesi distribution - North

Collection(s) - Koorders 18022

Weinmannia coodei H.C. Hopkins

Sulawesi distribution - South-East
Altitude $-1600 \mathrm{~m}$

Collection(s) - Coode 6197

Weinmannia descombesiana Bernardi

Sulawesi distribution - Central, South

Altitude - 1150-1800 m

Collection(s) - bb 20870, 22981, 24206, 26643; Eyma 432, 3590

Weinmannia devogelii H.C. Hopkins

Vernacular name(s) - Poeme

Sulawesi distribution - South

Altitude - 1-700 m

Collection(s) - Cel III 18; Darnaedi 2236; De Vogel 5682, 5959, 6122, 6234; Meijer 11147, 11181; Ramlanto 138; Schmid 5512; Van Balgooy 3625,3809

Weinmannia eymaeana H.C. Hopkins

Sulawesi distribution - Central

Lore Lindu National Park \& environs - present

Collection(s) - Eyma 3578

Weinmannia furfuracea H.C. Hopkins

Sulawesi distribution - Central

Lore Lindu National Park \& environs - present Altitude - 1700-2000 m

Collection(s) - Tantra 1587, 1595; Van Balgooy 3255,3464

Weinmannia spec.

Sulawesi distribution - South

Altitude - 1550-2700 m

Collection(s) - Eyma 452; Monod de Froideville 119

\section{CYCADACEAE}

CYCAS

\section{Cycas spec.}

Sulawesi distribution - South

Altitude - $500 \mathrm{~m}$

Collection(s) - Hennipman 6133; Van Balgooy 3930 


\section{DAPHNIPHYLLACEAE}

DAPHNIPHYLLUM

\section{Daphniphyllum glaucescens Blume}

Sulawesi distribution - South

Collection(s) - Teijsmann HB 14064, HB 14128

Daphniphyllum glaucescens Blume var. blumeanum (Baill. ex Müll.Arg.) Sm.

Sulawesi distribution - North

Altitude - $1200 \mathrm{~m}$

Collection(s) - Koorders 1684

\section{Daphniphyllum gracile Gagnep.}

Sulawesi distribution - South

Altitude $-2800 \mathrm{~m}$

Collection(s) - Eyma 1353

\section{Daphniphyllum spec.}

Sulawesi distribution - Central

Lore Lindu National Park \& environs - present

Altitude $-2200 \mathrm{~m}$

Collection(s) - Van Balgooy 3281

\section{DATISCACEAE}

\section{OCTOMELES}

\section{Octomeles sumatrana Miq.}

Vernacular name(s) - Benoa [Uma]; Binoea motoetoe

Sulawesi distribution - North, Central, South

Altitude $-25-1000 \mathrm{~m}$

Collection(s) - bb 24995, 32447; Burley, Partomihardjo \& Rachman 3787; Cel II 388; De Vogel \& Vermeulen 6659; De Vriese \& Teijsmann 135; Koorders 16697, 16698, 16699, 16702, 16703, 16704, 16705, 16706; Meijer 9409; Van Balgooy 3521; Whitmore \& Sidiyasa TCW 3447

\section{TETRAMELES}

Tetrameles nudiflora R.Br.

Vernacular name(s) - Paloemba; Wonolita

Sulawesi distribution - North, Central, South, South-East

Altitude - 3-600 m
Collection(s) - bb 6043, 21338, 23900, 24993, 29461; Cel V 175; Koorders 16707, 18698

\section{DICHAPETALACEAE}

\section{DICHAPETALUM}

Dichapetalum steenisii Leenh. subsp. celebicum Leenh.

Sulawesi distribution - South-East

Altitude - 150-497 m

Collection(s) - Elbert 3135, 3215

Dichapetalum timoriense (DC.) Boerl.

Sulawesi distribution - Central, South-East Altitude $-0-200 \mathrm{~m}$

Collection(s) - Elbert 3291; Eyma 3502; Kaudern 441

Dichapetalum spec.

Sulawesi distribution - North

Collection(s) - Meijer 10076A

\section{DILLENIACEAE}

\section{DILLENIA}

Dillenia celebica Hoogland

Sulawesi distribution - North, South

Altitude - 320-850 m

Collection(s) - bb 13755; Meijer 11212; Ramlanto 180; Van Balgooy 3770, 3802; Yusuf \& Wahyono 118

Dillenia ochreata (Miq.) Teijsm. \& Binn. ex Mart.

Vernacular name(s) - Djongi [Minahasa]

Sulawesi distribution - North

Altitude $-0-700 \mathrm{~m}$

Collection(s) - bb 17563; De Vogel 2448; Forman 226; Koorders 16710, 16712, 16714; Whitmore \& Sidiyasa TCW 3494, TCW 3532

Dillenia pentagyna Roxb.

Vernacular name(s) - Fanro

Sulawesi distribution - South, South-East

Altitude $-450 \mathrm{~m}$

Collection(s) - Bünnemeyer 12558; Meijer 10744; Noerkas 296; Rant 433 
Dillenia serrata Thunb.

Vernacular name(s) - Buah dingin; Buah Dongi; Dengilo; Dongi; Kalamur [Tonembuan]; Sonik; Woeadengi

Sulawesi distribution - North, Central, South, South-East

Altitude - 2-1500 m

Collection(s) - Alston 16538; bb 3924, 7505, 13747, 21131; Cel III 39; Coode 6288; De Vogel 5067, 5710; Ham L 7; Meijer 9597, 10056; Prawiroatmodjo \& Maskuri 1368; Prawiroatmodjo \& Soewoko 1794; Van Balgooy 3019

\section{TETRACERA}

Tetracera korthalsii Miq.

Sulawesi distribution - Central, South

Lore Lindu National Park \& environs - present

Altitude $-700 \mathrm{~m}$

Collection(s) - bb 2380; Teijsmann HB 13582

Tetracera nordtiana F. Muell. var. celebica Hoogland

Sulawesi distribution - South-East

Altitude - $200 \mathrm{~m}$

Collection(s) - Elbert 3382

Tetracera scandens (L.) Merr.

Sulawesi distribution - South, South-East

Altitude - 0-60 m

Collection(s) - Amir 24; Elbert 2975; Noerkas 162; Teijsmann HB 12602

\section{DIPTEROCARPACEAE}

\section{ANISOPTERA}

Anisoptera thurifera Blume

Sulawesi distribution - South

Altitude - 30-100 m

Collection(s) - bb 23910, 32468, 32611

\section{HOPEA}

\section{Hopea celebica Burck}

Vernacular name(s) - Dama dere item; Dama mata kucing, Poöti

Sulawesi distribution - South, South-East

Altitude $-0-500 \mathrm{~m}$
Collection(s) - bb 1826, 1905, 8569, 9350, 20522, 20524, 20525, 20526, 20527, 20529, 20530, 20531, 20533, 20534, 23839, 23918, 25535, 25554, 32526; Cel II 209, II 377; Kjellberg 2065; Meijer 10792, 11208, 11219 , 11257; Teijsmann HB 12779; Van Balgooy $3803,4040,4087$

\section{Hopea gregaria Slooten}

Vernacular name(s) - Poôti; Poöti

Sulawesi distribution - South-East

Altitude - 50-300 m

Collection(s) - bb 3885, 5001, 21750, 24071, 24072, 24073; Kjellberg 615

\section{SHOREA}

\section{Shorea assamica Dyer forma koordersii} (Brandis) Symington

Vernacular name(s) - Damar lari-lari

Sulawesi distribution - North, South

Altitude $-0-500 \mathrm{~m}$

Collection(s) - bb 21664, 21668, 23840, 23920, 32363, 32605, 33086, 33087, 33088; De Vriese \& Teijsmann 13; Koorders 15982, $16734,16736,18743$

\section{SUNAPTEA}

Sunaptea flavovirens (Slooten) Kosterm.

Vernacular name(s) - Tombopusu

Sulawesi distribution - Central, South

Altitude - 25-200 m

Collection(s) - bb 24973, 27053; Cel II 307, II 328 , II 330, II 468, IV 124

\section{VATICA}

\section{Vatica rassak (Korth.) Blume}

Vernacular name(s) - Damar deri; Dama deri hoetih; Lingkodo koetih; Noenoe

Sulawesi distribution - South

Altitude $-2-400 \mathrm{~m}$

Collection(s) - bb 8556, 21679, 23845, 23846, 23917, 32467, 32597; Cel III 12; Meijer 10726,11255 


\section{EBENACEAE}

\section{DIOSPYROS}

\section{Diospyros beccarii Hiern}

Sulawesi distribution - North

Collection(s) - Koorders 16753, 16760, 16765, 16770

Diospyros buxoides (Bakh.) Kosterm.

Sulawesi distribution - North, Central, South, South-East

Altitude - 15-200 m

Collection(s) - bb 21728, 31916, 32366; Ramlanto \& Fanani 568; Teijsmann HB 12286, HB 12319, HB 12873

\section{Diospyros cauliflora Blume}

Vernacular name(s) - Koeli maeto motoetoe Sulawesi distribution - North, South

Altitude $-10-300 \mathrm{~m}$

Collection(s) - Cel V 129, V 354, V 355, V 356, V 357; De Vogel 2441

Diospyros celebica Bakh.

Vernacular name(s) - Sora

Sulawesi distribution - North, Central, South

Altitude - 3-350 m

Collection(s) - bb 15576, 15577, 17635, 29468, 31480, 31915, 32461; Cel V 119, V 119a, V 403; Meijer 10837; Ramlanto 224

Diospyros eburnea Bakh.

Sulawesi distribution - North

Collection(s) - Reinwardt 89

Diospyros elliptica (J.R. Forst. \& G. Forst.) P.S. Green

Vernacular name(s) - Lada-lada [mewoeloe]

Sulawesi distribution - South, South-East

Altitude $-0-200 \mathrm{~m}$

Collection(s) - bb 22064; Cel V 245; Elbert 2583; Kjellberg 2362; Teijsmann HB 12397, HB 13586, HB 13645, HB 14066

Diospyros ferrea (Willd.) Bakh. var. buxifolia (Rottb.) Bakh.

Sulawesi distribution - North, South-East

Altitude - 150-497 m

Collection(s) - Elbert 3138; Koorders 16772
Diospyros ferrea (Willd.) Bakh. var. buxoides (Willd.) Bakh.

Sulawesi distribution - South

Collection(s) - Teijsmann HB 12085

Diospyros ferrea (Willd.) Bakh. var. glabrata (Warb.) Bakh.

Sulawesi distribution - South

Altitude $-350 \mathrm{~m}$

Collection(s) - Cel V 212

Diospyros ferrea (Willd.) Bakh. var. indonesica Bakh.

Vernacular name(s) - Moilo ajoe; Moilomo

Sulawesi distribution - North, Central

Lore Lindu National Park \& environs - present

Altitude - 8-300 m

Collection(s) - bb 15127, 16981, 18745, 19407 , $19644,30169,30170,31484$

Diospyros greshoffiana Koord. ex Bakh.

Sulawesi distribution - North

Collection(s) - Koorders 16746, 16750, 16764, 16767

\section{Diospyros macrocarpa Blume}

Sulawesi distribution - Central, South

Altitude $-15-300 \mathrm{~m}$

Collection(s) - bb 19815, 20324, 28727, 28733, 31817; Meijer 10788A

\section{Diospyros maritima Blume}

Sulawesi distribution - North, South, SouthEast

Altitude $-0-20 \mathrm{~m}$

Collection(s) - Alston 16096; bb 3687; Elbert 2556; Koorders 16757; Teijsmann HB 12144, HB 13910; Teijsmann \& De Vriese 25

Diospyros minahassae Bakh.

Sulawesi distribution — North, Central

Lore Lindu National Park \& environs - present Altitude $-280-600 \mathrm{~m}$

Collection(s) - Bloembergen 4233; Burley, Partomihardjo \& Rachman 3617; De Vogel 2465 
Diospyros peregrina (Gaertn.) Gurke forma javanica Kosterm.

Vernacular name(s) - Tagiboelabo

Sulawesi distribution - North, Central, South, South-East

Altitude - 3-180 m

Collection(s) - bb 6030, 19446, 21586, 23594, 24106, 24982, 31483, 32511; Koorders $16747,16754,16762$

Diospyros philippinensis A.DC.

Sulawesi distribution - North

Collection(s) - Koorders 16755

Diospyros pilosanthera Blume

Vernacular name(s) - Saoe mogito

Sulawesi distribution - North, South-East

Altitude - 3-200 m

Collection(s) - bb 5858, 19430, 19594, 20015, 21114, 32479; Koorders 16745, 16746, $16758,16763,16768$

\section{Diospyros rumphii Bakh.}

Sulawesi distribution - North

Collection(s) - Koorders 16743, 16761, 16769

\section{Diospyros spec.}

Sulawesi distribution - North, Central, SouthEast

Altitude - 20-2200 m

Collection(s) - bb 21770; Burley, Partomihardjo \& Rachman 3720, 3774, 3798; De Vogel \& Vermeulen 6581, 6597, 6792, 6934, 6981, 7053; McDonald \& Ismail 3897; Prance 30097; Prawiroatmodjo \& Soewoko 1767, 1874; Van Balgooy 3289

\section{ELAEAGNACEAE}

\section{ELAEAGNUS}

\section{Elaeagnus triflora Roxb.}

Sulawesi distribution - South

Altitude - 850-1500 m

Collection(s) - Bünnemeyer 11642; Eyma 1205; Yoshida 913

\section{ELAEOCARPACEAE}

\section{ELAEOCARPUS}

\section{Elaeocarpus angustifolius Blume}

Vernacular name(s) - Maningkoloe; Pengoe mongkoeni

Sulawesi distribution - North, Central, South, South-East

Altitude - 0-2050 m

Collection(s) - bb 15032, 17678, 20435, 21275, 21376, 26277; Cel V 204; Coode 5913, 5973, 6052; -Forman 263; Koorders 18979, 18981, 18991, 18992, 18994, 18998, 19001; Tantra 1554; Van Balgooy 3093

\section{Elaeocarpus balgooyi Coode}

Sulawesi distribution - South

Altitude $-300 \mathrm{~m}$

Collection(s) - Van Balgooy 3831

Elaeocarpus batui Coode

Sulawesi distribution - Central

Altitude $-70-80 \mathrm{~m}$

Collection(s) - Coode 6034

\section{Elaeocarpus burkii Coode}

Sulawesi distribution - South

Altitude $-2200 \mathrm{~m}$

Collection(s) - bb 23360

Elaeocarpus celebicus Koord.

Sulawesi distribution - North

Collection(s) - Koorders 18985, 18990, 18993, 18995, 18999

\section{Elaeocarpus culminicola Warb.}

Sulawesi distribution - North, South

Altitude $-250-850 \mathrm{~m}$

Collection(s) - bb 22990, 31818; Koorders 19011; Yusuf \& Wahyono 130

\section{Elaeocarpus cumingii Turcz.}

Vernacular name(s) - Katji; Mendoe kinaa

Sulawesi distribution - North, Central, South

Altitude - 25-200 m

Collection(s) - Cel I 51, V 257; Koorders 18986, 19003, 19006; Noerkas 228 


\section{Elaeocarpus dolichostylus Schltr.}

Vernacular name(s) - Antoala; Mata kuching Sulawesi distribution - North, Central

Altitude - 100-1080 m

Collection(s) - bb 28282; De Vogel 2505; Lam 2611; Van Balgooy 3138,3512

\section{Elaeocarpus erdinii Coode}

Sulawesi distribution - Central

Altitude $-1100-4000 \mathrm{~m}$

Collection(s) - Coode 6112,6114, 6121; Musser 929

\section{Elaeocarpus eymae Coode}

Sulawesi distribution - South

Altitude - 2800-3000 m

Collection(s) - Eyma 619

\section{Elaeocarpus gambutensis Coode}

Sulawesi distribution - North

Altitude $-1420 \mathrm{~m}$

Collection(s) - Koorders 19008; Milliken 955

Elaeocarpus harunii Coode

Sulawesi distribution - North

Altitude $-1900 \mathrm{~m}$

Collection(s) - Milliken 1082

Elaeocarpus kjellbergii Coode

Sulawesi distribution - Central

Altitude $-2500 \mathrm{~m}$

Collection(s) - Kjellberg 1478a

Elaeocarpus lancistipulatus Coode

Sulawesi distribution - North

Altitude $-1500 \mathrm{~m}$

Collection(s) - Milliken 1100

\section{Elaeocarpus linnaei Coode}

Sulawesi distribution - North

Altitude $-1700 \mathrm{~m}$

Collection(s) - Milliken 1069

Elaeocarpus littoralis Teysm. \& Binn. ex Kurz

Sulawesi distribution - South

Collection(s) - Kofman 261
Elaeocarpus macrocerus (Turcz.) Merr.

Vernacular name(s) - Tilaa poete laa

Sulawesi distribution - North, South

Altitude - 300-400 m

Collection(s) - Cel III 74; De Vogel 5847, 6387; Meijer 10141, 11430; Van Balgooy 3737

Elaeocarpus macrocerus (Turcz.) Merr. subsp. heinrichii (Kn.) Coode

Sulawesi distribution - South-East

Altitude - 20-250 m

Collection(s) - Prawiroatmodjo \& Soewoko 1800

Elaeocarpus macropus Warb.

Vernacular name(s) - Pai pai [Soroako]

Sulawesi distribution - North, South

Altitude $-450 \mathrm{~m}$

Collection(s) — De Vogel 6054; Koorders 18983

Elaeocarpus macropus Warb. var. macropus

Vernacular name(s) - Ilokopoe

Sulawesi distribution - Central, South, SouthEast

Altitude - 20-1000 m

Collection(s) - Cel II 447; Meijer 11131; Prawiroatmodjo \& Soewoko 1752, 1845; Van Balgooy 3542

Elaeocarpus multiflorus (Turcz.) Villar

Vernacular name(s) - antamat'a; antaola

Sulawesi distribution - North, Central, South Altitude - 5-1000 m

Collection(s) - bb 13902, 24702; Koorders 19000, 19004, 19007; Lam 3120, 3444

Elaeocarpus musseri Coode

Sulawesi distribution - Central, South, SouthEast

Lore Lindu National Park \& environs - present Altitude - 400-1800 m

Collection(s) - Coode 6144; Van Balgooy 3180, 4070; Yusuf \& Wahyono 50

Elaeocarpus octopetalus Merr.

Vernacular name(s) - Koelingka

Sulawesi distribution - North, South, SouthEast

Altitude $-10-500 \mathrm{~m}$ 
Collection(s) - bb 5425, 21724; Cel II 221; Darnaedi 2256; De Vogel 5808, 6025, 6139; Koorders 18987, 18996, 19029; Ramlanto 209; Van Balgooy 3654, 3826; Van Vuuren 441

\section{Elaeocarpus petiolatus Jack}

Sulawesi distribution - South, South-East Altitude - 100-400 m

Collection(s) - Amir 170; De Vogel 6198

Elaeocarpus purus Coode

Sulawesi distribution - Central

Altitude $-2000 \mathrm{~m}$

Collection(s) - Lack \& Grimes 1737

Elaeocarpus teijsmannii Koord. \& Valeton

Vernacular name(s) - Kaluku [Soroako]; Pingoe Sulawesi distribution - North, South

Altitude - 0-1600 m

Collection(s) - bb 22993, 26299; Burley, Partomihardjo \& Rachman 3784; Cel III 11; De Vogel 2655, 6070; Forbes 1185A; Koorders 18982, 18988, 19005, 19617; Van Balgooy $3720,3873,4005$

Elaeocarpus teijsmannii Koord. \& Valeton subsp. domatiferus Coode

Sulawesi distribution - North

Altitude $-1500 \mathrm{~m}$

Collection(s) - Milliken 1099

Elaeocarpus teijsmannii Koord. \& Valeton subsp. morowalensis Coode

Sulawesi distribution - Central

Collection(s) — Lack \& Grimes 1668

Elaeocarpus teijsmannii Koord. \& Valeton subsp. rhizophorus (Koord.) Coode

Sulawesi distribution - North

Altitude - $1200 \mathrm{~m}$

Collection(s) - Koorders 18989

\section{Elaeocarpus spec.}

Sulawesi distribution - North, Central, South, South-East

Altitude $-2450 \mathrm{~m}$

Collection(s) - bb 14949, 26636, 26638, 28294; Coode 6153; De Vogel 7215; Keßler et al. PK 3163; Koorders 18984, 18997; Meijer 10757; Van Balgooy 3320

\section{SLOANEA}

Sloanea celebica Boerl. \& Koord.

Sulawesi distribution - North, South

Altitude - 700-1100 m

Collection(s) - bb 19705; Koorders 19030

Sloanea javanica (Miq.) Koord. \& Valeton

Sulawesi distribution - South

Altitude $-200 \mathrm{~m}$

Collection(s) - bb 19690

\section{ERICACEAE}

\section{DIPLYCOSIA}

Diplycosia aperta J.J. Sm.

Sulawesi distribution - Central

Altitude $-2500-2800 \mathrm{~m}$

Collection(s) - Kjellberg 1454, 3923

Diplycosia capitata Sleumer

Sulawesi distribution - South

Collection(s) - K.F. Sarasin \& P.B. Sarasin 2081

Diplycosia caryophylloides J.J. Sm.

Sulawesi distribution - South

Altitude $-1500 \mathrm{~m}$

Collection(s) - Kjellberg 3914

Diplycosia caryophylloides J.J. Sm. var. longipes Sleumer

Sulawesi distribution - North

Collection(s) - Eyma 3960

Diplycosia crassiramea Sleumer

Sulawesi distribution - South

Altitude - $3000 \mathrm{~m}$

Collection(s) - Eyma 736

\section{Diplycosia filipes Sleumer}

Sulawesi distribution - South

Altitude $-2800 \mathrm{~m}$

Collection(s) - Eyma 1409

Diplycosia haemantha Sleumer

Sulawesi distribution - North 
Collection(s) - Eyma 3577

Diplycosia kjellbergii J.J. Sm.

Sulawesi distribution - South

Altitude - $1500 \mathrm{~m}$

Collection(s) - Kjellberg 3913

Diplycosia minutiflora Sleumer

Sulawesi distribution - North

Altitude $-1800 \mathrm{~m}$

Collection(s) - Steup 21

Diplycosia retusa Sleumer

Sulawesi distribution - Central

Altitude - 1300-1700 m

Collection(s) - K.F. Sarasin \& P.B. Sarasin 2097

Diplycosia rubidiflora J.J. Sm.

Sulawesi distribution - South

Altitude $-1900 \mathrm{~m}$

Collection(s) - Bünnemeyer 12153

Diplycosia sagittanthera J.J. Sm.

Sulawesi distribution - South

Altitude $-800 \mathrm{~m}$

Collection(s) - Kjellberg 2316

Diplycosia stenophylla Sleumer

Sulawesi distribution - North

Altitude - 1700-1800 m

Collection(s) - Eyma 1603

Diplycosia triangulanthera J.J. Sm.

Sulawesi distribution - South

Altitude $-1000 \mathrm{~m}$

Collection(s) - Kjellberg 1811

Diplycosia undata J.J. Sm.

Sulawesi distribution - South

Altitude $-2850 \mathrm{~m}$

Collection(s) - Bünnemeyer 12222

\section{GAULTHERIA}

Gaultheria celebica J.J. Sm.

Sulawesi distribution - South

Collection(s) - Rachmat 887
Gaultheria celebica J.J. Sm. var. petiolata J.J. Sm.

Sulawesi distribution - South

Altitude $-2400 \mathrm{~m}$

Collection(s) - Bünnemeyer 12394

Gaultheria viridiflora Sleumer

Sulawesi distribution - South

Altitude - $2900 \mathrm{~m}$

Collection(s) - Eyma 957

\section{RHODODENDRON}

Rhododendron alternans Sleumer

Sulawesi distribution - South

Altitude $-2860 \mathrm{~m}$

Collection(s) - Eyma 1363

Rhododendron amabile Sleumer

Sulawesi distribution - South

Altitude $-1700 \mathrm{~m}$

Collection(s) - Steup 206

Rhododendron arenicolum Sleumer

Sulawesi distribution - South

Altitude $-2900 \mathrm{~m}$

Collection(s) - Eyma 955; Sands 453

Rhododendron bloembergenii Sleumer

Sulawesi distribution - North, Central, South Lore Lindu National Park \& environs - present

Collection(s) - Bloembergen 3972; Meijer 9901; Van Balgooy 3311

Rhododendron celebicum DC.

Sulawesi distribution - South

Collection(s) - Bogor Botanical Garden 881, 882; Kofman 238

Rhododendron eymae Sleumer

Sulawesi distribution - South

Altitude $-3300 \mathrm{~m}$

Collection(s) - Eyma 684; Sands 228

Rhododendron lagunculicarpum J.J. Sm.

Sulawesi distribution - Central, South

Altitude $-3000 \mathrm{~m}$

Collection(s) - Eyma 649; Sands 230, 279 
Rhododendron leptobrachion Sleumer

Sulawesi distribution - Central

Altitude - 2800-3000 m

Collection(s) — Eyma 647

Rhododendron leptomorphum Sleumer

Sulawesi distribution - North

Collection(s) - Eyma 3617

Rhododendron lindaueanum Koord. var. bantaengense J.J. Sm.

Sulawesi distribution - South

Altitude - $2890 \mathrm{~m}$

Collection(s) - Bünnemeyer 12250

Rhododendron lompohense J.J. Sm.

Sulawesi distribution - Central

Collection(s) - Rachmat 943

Rhododendron nanophyton Sleumer

Sulawesi distribution - South

Altitude - 3100-3300 m

Collection(s) - Eyma 682

Rhododendron nanophyton Sleumer var. saxicolum Sleumer

Sulawesi distribution - South

Altitude - 3200-3250 m

Collection(s) - Eyma 1046

Rhododendron poremense J.J. Sm.

Sulawesi distribution - South-East

Altitude - $1400 \mathrm{~m}$

Collection(s) - Kjellberg 2627

Rhododendron pseudobuxifolium Sleumer

Sulawesi distribution - South

Collection(s) - Eyma 866

Rhododendron pubitubum Sleumer

Sulawesi distribution - South

Altitude $-2000 \mathrm{~m}$

Collection(s) - Eyma 1441; Steup 204

Rhododendron pudorinum Sleumer

Sulawesi distribution - South

Altitude $-3000 \mathrm{~m}$

Collection(s) - Eyma 646; Sands 244
Rhododendron quadrasianum S. Vidal var. selebicum J.J. Sm.

Sulawesi distribution - South

Altitude $-0-2700 \mathrm{~m}$

Collection(s) - Monod de Froideville 120; Rachmat 933

Rhododendron radians J.J. Sm. var. minahasae Sleumer

Sulawesi distribution - North

Altitude $-1700 \mathrm{~m}$

Collection(s) - Boesveld 6

Rhododendron rhodopus Sleumer

Sulawesi distribution - Central, South

Altitude $-3000 \mathrm{~m}$

Collection(s) - Eyma 643; Wieringa 1882

Rhododendron scarlatinum Sleumer

Sulawesi distribution - South

Altitude $-2950 \mathrm{~m}$

Collection(s) - Eyma 782

Rhododendron vanvuurenii J.J. Sm.

Sulawesi distribution - South

Collection(s) - Eyma 1147; Monod de Froideville 398; Rachmat 878

Rhododendron zollingeri J.J. Sm.

Sulawesi distribution - South

Collection(s) - Monod de Froideville 271; Rachmat 883; Sands 277

\section{VACCINIUM}

Vaccinium apophysatum Sleumer

Sulawesi distribution - North

Altitude $-400 \mathrm{~m}$

Collection(s) - Lam 3274

Vaccinium aucupis Sleumer

Sulawesi distribution - South

Altitude - $1500 \mathrm{~m}$

Collection(s) - Heinrich 332

Vaccinium centrocelebicum Sleumer

Sulawesi distribution - South

Altitude - 2600-3000 m

Collection(s) - Eyma 586 
Vaccinium centrocelebicum Sleumer var. maius Sleumer

Sulawesi distribution - South

Altitude $-2400 \mathrm{~m}$

Collection(s) - bb 26640

Vaccinium contractum Sleumer

Sulawesi distribution - Central

Altitude - $1630 \mathrm{~m}$

Collection(s) - K.F. Sarasin \& P.B. Sarasin 2080

Vaccinium cuneiflolium Miq.

Sulawesi distribution - North

Collection(s) - Reinwardt 1566

Vaccinium dubiosum J.J. Sm.

Sulawesi distribution - Central

Altitude $-800 \mathrm{~m}$

Collection(s) - Kjellberg 3969

Vaccinium kjellbergii J.J. Sm.

Sulawesi distribution - South, South-East

Altitude - 0-900 m

Collection(s) - Elbert 3480; Kjellberg 2068

Vaccinium latissimum J.J. Sm.

Sulawesi distribution - South

Collection(s) - Wieringa 1894

Vaccinium paludicolum Sleumer

Sulawesi distribution - North

Collection(s) - Eyma 3957

Vaccinium sclerophyllum Sleumer

Sulawesi distribution - North

Collection(s) - Eyma 3623

Vaccinium tomicipes J.J. Sm.

Sulawesi distribution - Central

Altitude - $2500 \mathrm{~m}$

Collection(s) - Kjellberg 1453

Vaccinium warburgii Sleumer

Sulawesi distribution - South

Altitude $-3000 \mathrm{~m}$

Collection(s) - Warburg 16811

\section{Vaccinium spec.}

Vernacular name(s) - Copeng [Timampu]; Wolisi [Sulawesi Tenggara]

Sulawesi distribution - North, Central, South, South-East

Altitude - 20-2900 m

Collection(s) - Burley, Partomihardjo \& Rachman 3755; Coode 6184; De Vogel 5247, 5354, 5356, 5368, 5395, 5425, 5426, 5437 . 5607, 5804, 5818, 5829, 6324, 6344; Eyma 3795; Hennipman 5345, 6053; Kofman 225, 254; McDonald \& Ismail Cat 4153; Monod de Froideville 125; Sands 290, 584; Tantra 1567, 1601; Van Balgooy 3233, 3364, 3395, 3443, 3688; Yoshida 1187, 1448; Yusuf \& Wahyono 161

\section{ERYTHROXYLACEAE}

\section{ERYTHROXYLUM}

Erythroxylum burmanicum Griff.

Sulawesi distribution - North, Central

Altitude $-50-400 \mathrm{~m}$

Collection(s) - De Joncheere 1031; Meijer 10177; Van Balgooy 2963

Erythroxylum cuneatum (Miq.) Kurz var. bancanum Schulz

Sulawesi distribution - North

Collection(s) - Alston 15589

Erythroxylum ecarinatum Burck

Sulawesi distribution - Central, South, SouthEast

Altitude - 20-300 m

Collection(s) - bb 31888; Cel II 267; Prawiroatmodjo \& Soewoko 1881, 1938

Erythroxylum novogranatense (Morris) Hieron.

Sulawesi distribution - North

Altitude $-750 \mathrm{~m}$

Collection(s) - Kaudern 2 


\section{EUPHORBIACEAE}

ACALYPHA

\section{Acalypha amentacea Roxb.}

Sulawesi distribution - North, Central Altitude - 0-800 m

Collection(s) - Eyma 3512; Kaudern 295, 396; Lam 2497, 2567, 3358; Meijer 9257, 10197; Noerkas 379; Posthumus 2375; Van Balgooy 3608,3620

Acalypha capillipes Müll. Arg.

Sulawesi distribution - North, South-East

Altitude $-0-300 \mathrm{~m}$

Collection(s) - Elbert 2946

\section{Acalypha caturus Blume}

Sulawesi distribution - North, Central, South, South-East

Altitude - 0-1400 m

Collection(s) - bb 14148; Bloembergen 4138, 4217, 4248; Bünnemeyer 12553; Cel V 263; Coode 5991; De Vogel 2490; Elbert 3389, 3450; Eyma 1155; Johansson 573; Koorders 16779, 16780, 16781, 16787, 19464; Lam 2766; Manichit 26; Meijer 10043, 10709; Milliken 908; Noerkas 448; Rachmat 152, 570; Ramlanto 259; Ramlanto \& Fanani 491; Teijsmann HB 12834, HB 14177; Van Balgooy 3506

Acalypha grandis Benth.

Sulawesi distribution - North

Collection(s) - Koorders 16784, 16786, 16867

Acalypha hellwigii Warb.

Sulawesi distribution - North

Collection(s) - Eyma 3750

Acalypha hellwigii Warb. var. mollis (Warb.) K. Schum. \& Lauterb.

Sulawesi distribution - Central, South Lore Lindu National Park \& environs - present Collection(s) - Meijer 9247; Yoshida 1425

Acalypha indica $\mathrm{L}$.

Sulawesi distribution - South

Collection(s) - Koorders 16778; Noerkas 1
Acalypha lanceolata Willd.

Sulawesi distribution - Central, South, SouthEast

Altitude - 0-400 m

Collection(s) - Elbert 2883, 2925; Kaudern 373; Monod de Froideville 389; Noerkas 5

Acalypha longispica Warb.

Sulawesi distribution - Central

Altitude - $1000 \mathrm{~m}$

Collection(s) - De Vogel 5069, 5194

Acalypha spec.

Sulawesi distribution - North, Central, South Lore Lindu National Park \& environs - present Altitude - 0-1060 m

Collection(s) - Alston 15659, 15722, 16104; Coode 5859, 5965; Eyma 1539, 3751; Johansson 160, 337; Keßler et al. PK 3145; Meijer 10198; Whitmore \& Sidiyasa TCW 3374 , TCW 3515

\section{ACTEPHILA}

Actephila javanica Miq.

Sulawesi distribution - North

Collection(s) - Koorders 16937

Actephila spec.

Sulawesi distribution - Central, South

Altitude - 300-450 m

Collection(s) - Coode 5916; Yusuf \& Wahyono 13

\section{ALCHORNEA}

Alchornea rugosa (Lour.) Müll. Arg.

Sulawesi distribution - North, South-East

Altitude $-0-750 \mathrm{~m}$

Collection(s) - Burley et al. 3993; Koorders 16807, 16951; Prawiroatmodjo \& Maskuri $1108,1200,1477$

\section{ALEURITES}

Aleurites moluccana (L.) Willd.

Sulawesi distribution - North, South, SouthEast

Altitude $-0-560 \mathrm{~m}$ 
Collection(s) - bb 21348, 21381; Bünnemeyer 12486; Chin 3434; Elbert 2603, 2603a; Koorders 16805; Prawiroatmodjo \& Maskuri 1560, 1579; Prawiroatmodjo \& Soewoko 1823; Rachmat 376

\section{ANTIDESMA}

Antidesma bunius (L.) Spreng.

Sulawesi distribution - South

Collection(s) - Rachmat 617

Antidesma celebicum Miq.

Sulawesi distribution - North, Central, South, South-East

Altitude - 0-1200 m

Collection(s) - Alston 16492; bb 14329, 17044; Burley, Partomihardjo \& Rachman 3550, 3643; Burley et al. 3558; Cel V 125; De Vogel \& Vermeulen 6555; Elbert 3162; Forman 253, 335; Johansson 386; Koorders 16790, 16792, 16793, 16795, 16799, 16800; Lam 2785, 3379; Meijer 9719, 10686; Milliken 917; Noerkas 498; Teijsmann HB 12693

Antidesma coriaceum Tul.

Sulawesi distribution - North

Altitude $-700 \mathrm{~m}$

Collection(s) - Forman 316

Antidesma elbertii Petra Hoffm.

Sulawesi distribution - North, Central, South, South-East

Altitude - 0-650 m

Collection(s) - Cel III 32; Coode 5838, 5844, 6068; De Vogel 5626; De Vogel \& Vermeulen 6508, 6517; Elbert 3079; Meijer 10811; Prawiroatmodjo \& Maskuri 1233, 1417; Prawiroatmodjo \& Soewoko 1887; Rachmat 755; Whitmore \& Sidiyasa TCW 3465

Antidesma excavatum J.J. Sm.

Sulawesi distribution - North, Central, South Altitude - 0-1050 m

Collection(s) - De Vogel 5612; Meijer 11289; Rachmat 641

\section{Antidesma ghaesembilla Gaertn.}

Sulawesi distribution - Central, South

Altitude - 0-400 m
Collection(s) - Amir 16; bb 20328; Noerkas 217; Prawiroatmodjo \& Maskuri 1201; Teijsmann HB 12362, HB 12661; Van Balgooy 3936

\section{Antidesma heterophyllum Blume}

Sulawesi distribution - South-East

Altitude $-0-5 \mathrm{~m}$

Collection(s) - Elbert 2553

\section{Antidesma montanum Blume}

Sulawesi distribution - North, Central, South, South-East

Altitude - 0-497 m

Collection(s) - Burley, Partomihardjo \& Rachman 4209, 4219; Chin 3392; Coode 5879, 6242; Elbert 3139; Ramlanto \& Fanani 675

Antidesma riparium Airy Shaw

Sulawesi distribution - Central, South, SouthEast

Altitude - 20-1000 m

Collection(s) - Meijer 9933; Prawiroatmodjo \& Soewoko 1979; Tantra 1537; Van Balgooy 3130

\section{Antidesma stipulare Blume}

Sulawesi distribution - North, Central, South, South-East

Altitude - 0-750 m

Collection(s) - Afriastini 2002; Burley, Partomihardjo \& Rachman 4222; Johansson 62, 123; Meijer 10129; Prawiroatmodjo \& Maskuri 1462; Prawiroatmodjo \& Soewoko 1891

Antidesma tomentosum Blume

Sulawesi distribution - North, Central

Altitude - 70-500 m

Collection(s) - Coode 5948; Koorders 16854; Lam 2693, 3298

\section{Antidesma spec.}

Sulawesi distribution - North, South, SouthEast

Altitude - 0-1400 m

Collection(s) - Coode 6138; Koorders 16802; Mamahit 27 
APOROSA

\section{Aporosa falcifera Hook.f.}

Sulawesi distribution - North

Altitude $-500 \mathrm{~m}$

Collection(s) - Koorders 16798, 16801, 16973

Aporosa frutescens Blume

Sulawesi distribution - North, Central

Altitude $-750 \mathrm{~m}$

Collection(s) - Burley, Partomihardjo \& Rach$\operatorname{man} 3520,3556,3730,3840,4023,4044$; Ramlanto \& Fanani 666

Aporosa leytensis Merr.

Sulawesi distribution - North, Central

Altitude - 250-750 m

Collection(s) - bb 14341, 14543; De Vogel \& Vermeulen 6770; Johansson 198; Whitmore \& Sidiyasa TCW 3472, TCW 3493

Aporosa lucida (Miq.) Airy Shaw

Sulawesi distribution - South

Altitude $-480 \mathrm{~m}$

Collection(s) - Kofman 134

Aporosa octandra (Buch.-Ham. ex D. Don) Vickery var. malesiana Schot

Sulawesi distribution - Central, South, SouthEast

Altitude $-150 \mathrm{~m}$

Collection(s) - bb 5851, 21612, 24974; Prawiroatmodjo \& Maskuri 1265; Rachmat 837, 847

\section{AUSTROBUXUS}

Austrobuxus nitidus Miq.

Sulawesi distribution - South, South-East

Altitude $-300-1700 \mathrm{~m}$

Collection(s) - De Vogel 6391, 6392; McDonald \& Ismail 3861, 4055; Neth. Ind. For. Service 228; Van Balgooy 4086, 4089

\section{BACCAUREA}

Baccaurea javanica Müll. Arg.

Sulawesi distribution - North, Central, South Altitude $-80 \mathrm{~m}$
Collection(s) - Koorders 16809, 16811, 16813, $16814,16815,16816,16848$; Lam 2657, 2739; Meijer 10814; Wardi Wd 106

\section{Baccaurea nanihua Merr.}

Sulawesi distribution - South, South-East

Altitude - $645 \mathrm{~m}$

Collection(s) - bb 9711, 23572, 26013; Cel II 440; Prawiroatmodjo \& Soewoko 1736, 1766; Van Balgooy 3834

\section{Baccaurea odoratissima Elmer}

Sulawesi distribution - North

Altitude - 350-750 m

Collection(s) - Burley, Partomihardjo \& Rach$\operatorname{man} 3604$

Baccaurea papuana F.M. Bailey

Sulawesi distribution - North

Altitude $-150 \mathrm{~m}$

Collection(s) - Burley, Partomihardjo \& Rach$\operatorname{man} 4047,4101,4202,4244$

Baccaurea pubera (Miq.) Müll.Arg.

Sulawesi distribution - South

Collection(s) - Meijer 11222

Baccaurea pyriformis Gage

Sulawesi distribution - Central

Altitude $-250 \mathrm{~m}$

Collection(s) - Ramlanto \& Fanani 652

Baccaurea racemosa (Reinw, ex Blume) Müll.Arg.

Sulawesi distribution - North, Central, South Altitude $-1600 \mathrm{~m}$

Collection(s) - bb 24198; De Vogel \& Vermeulen 6769; Forman 255; Johansson 69; Meijer 10995; Ramlanto \& Fanani 533

Baccaurea tetrandra (Baill.) Müll.Arg.

Sulawesi distribution - North, Central, SouthEast

Altitude $-750 \mathrm{~m}$

Collection(s) - Burley, Partomihardjo \& Rachman 3532, 3634, 3890, 3936; Coode 6079, 6082; Prawiroatmodjo \& Soewoko 1973; Wardi Wd 107 


\section{BISCHOFLA}

\section{Bischofia javanica Blume}

Sulawesi distribution - North, Central Altitude $-650 \mathrm{~m}$

Collection(s) - Alston 15826; bb 19451, 23895, 25005, 32489; De Vogel 5643; De Vogel \& Vermeulen 6633; Koorders 16817, 16818; Lam 2901; Noerkas 378

\section{BLUMEODENDRON}

Blumeodendron kurzii (Hook.f.) J.J. Sm.

Sulawesi distribution - North

Altitude $-150 \mathrm{~m}$

Collection(s) - Burley, Partomihardjo \& Rachman 4104

\section{BREYNIA}

Breynia cernua (Poir.) Müll. Arg.

Sulawesi distribution - North, Central, South, South-East

Altitude $-1250 \mathrm{~m}$

Collection(s) - Bloembergen 4036; Burley et al. 3982; De Vogel 2513, 5712, 5723, 5885, 6057; Coode 6292; Elbert 2789, 3009, 3391, 3437, 3439; Eyma 1136, 3732; Forman 345; Koorders 16878, 16940, 16941, 16961; Lam 2933; McDonald \& Ismail 4102; Meijer 10650, 10736; Milliken 941; Noerkas 321; Prawiroatmodjo \& Soewoko 1629; Rachmat 10; Ramlanto 235; Widjaja 766

Breynia microphylla (Kurz ex Teijsm. \& Binn.) Müll.Arg.

Sulawesi distribution - South

Altitude - $1600 \mathrm{~m}$

Collection(s) - Bünnemeyer 12138; Elbert 2584; Mamahit 31; Teijsmann HB 14013; Yoshida 1211

\section{BRIDELIA}

\section{Bridelia glauca Blume}

Sulawesi distribution - North, South

Altitude $-0-850 \mathrm{~m}$

Collection(s) - Bloembergen 4229; Koorders $16820,16821,16823,16826,16832$; Yusuf \& Wahyono 47

\section{Bridelia insulana Hance}

Sulawesi distribution - North, Central, South, South-East

Altitude $-0-500 \mathrm{~m}$

Collection(s) - bb 16650, 19409, 19449, 21749 , 31490, 31875; Cel III 65; Forman 406; Koorders 16822, 16824, 16825, 16828, 16829 , 16830, 16831; Teijsmann HB 5593, HB 12819

\section{Bridelia tomentosa Blume}

Sulawesi distribution - Central, South, SouthEast

Lore Lindu National Park \& environs - present Altitude - 0-950 m

Collection(s) - Bünnemeyer 11284; De Vogel 5664, 5665; Elbert 2573, 3380; Eyma 330; McDonald \& Ismail 4038; Meijer 10214; Rachmat 31; Teijsmann HB 11768, HB 12115, HB 12167, HB 12292

\section{CHAMAESYCE}

Chamaesyce vachellii (Hook. \& Arn.) Hurus.

Sulawesi distribution - North, South, SouthEast

Altitude - 0-300 m

Collection(s) - De Vogel 6378; Eyma 343, 370, 3379; Kaudern 279; Kjellberg 166; Monod de Froideville 396; Rachmat 100; Teijsmann HB 11923; Zollinger 2308

\section{CLADOGYNOS}

Cladogynos orientalis Zipp. ex Span.

Sulawesi distribution - Central, South-East Lore Lindu National Park \& environs - present Altitude $-280 \mathrm{~m}$

Collection(s) - Coode 5863; Kaudern 490; Kjellberg 375; Van Balgooy 2991

\section{CLAOXYLON}

Claoxylon brachyandrum Pax \& K. Hoffm.

Sulawesi distribution - South

Altitude $-2400 \mathrm{~m}$

Collection(s) - Bünnemeyer 12025, 12111, 12321 


\section{Claoxylon frutescens Airy Shaw}

Sulawesi distribution - North, South

Collection(s) - Koorders 16835, 16966; Teijsmann HB 14043

Claoxylon longifolium (Blume) Endl. ex Hassk.

Sulawesi distribution - South

Altitude $-200 \mathrm{~m}$

Collection(s) - Cel V 261

Claoxylon polot (Burm.f.) Merr.

Sulawesi distribution - North, South-East Altitude $-200 \mathrm{~m}$

Collection(s) - Elbert 3313

Claoxylon purpureum Merr.

Sulawesi distribution - South

Altitude $-2200 \mathrm{~m}$

Collection(s) - Bünnemeyer 11850, 11856

\section{CLEIDION}

Cleidion javanicum Blume

Sulawesi distribution - North

Collection(s) - Koorders 16938

\section{CLEISTANTHUS}

Cleistanthus myrianthus (Hassk.) Kurz

Sulawesi distribution - North, South

Altitude $-0-500 \mathrm{~m}$

Collection(s) - Cel I 74, II 326, II 487; Koorders 16919; Lam 2900, 2980, 3337; Meijer 10705; Teijsmann 12427, HB 11746, HB 11882, HB 12409, HB 12573, HB 12634, HB 12686

Cleistanthus sumatranus (Miq.) Müll.Arg.

Sulawesi distribution - North, Central, South, South-East

Altitude $-0-250 \mathrm{~m}$

Collection(s) - bb 24975; Cel IV 106, V 297; Elbert 3004; Koorders 16920; Prawiroatmodjo \& Maskuri 1439, 1440; Prawiroatmodjo \& Soewoko 1704; Rachmat 263; Teijsmann HB 12000, HB 12583, HB 12799

\section{Cleistanthus spec.}

Sulawesi distribution - Central, South-East Altitude $-0-320 \mathrm{~m}$
Collection(s) - Coode 5907; Elbert 2762; Prawiroatmodjo \& Maskuri 1258, 1461

CODIAEUM

Codiaeum variegatum (L.) Blume

Sulawesi distribution - North

Altitude $-0-200 \mathrm{~m}$

Collection(s) - Lam 2888, 2993, 3013

\section{CROTON}

\section{Croton argyratus Blume}

Sulawesi distribution - North, South

Altitude - 0-500 m

Collection(s) - Koorders 16838; Teijsmann HB 11878

Croton cascarilloides Raeusch.

Sulawesi distribution - South-East

Altitude - 250-320 m

Collection(s) - Elbert 2719

\section{Croton caudatus Geiseler}

Sulawesi distribution - Central Lore Lindu National Park \& environs - present Collection(s) - Meijer 9200

Croton oblongus Burm.f.

Sulawesi distribution - North, South

Altitude $-0-300 \mathrm{~m}$

Collection(s) - Bünnemeyer 10665; Koorders 19643; Lam 3277

\section{Croton tiglium L.}

Sulawesi distribution - North, Central, South Altitude $-0-600 \mathrm{~m}$

Collection(s) - Kaudern 418; Koorders 16840, 16841; Noerkas 420; Rachmat 310

Croton verreauxii Müll.Arg.

Sulawesi distribution - South

Collection(s) - Meijer 10948

\section{Croton spec.}

Sulawesi distribution - South

Altitude - $400 \mathrm{~m}$

Collection(s) — De Vogel 6156 


\section{DRYPETES}

Drypetes celebica (Boerl. \& Koord.) Pax \& K. Hoffm.

Sulawesi distribution - North, South

Altitude - 0-400 m

Collection(s) - bb 33119; Koorders 16774; Teijsmann HB 12295

Drypetes celebica (Boerl. \& Koord.) Pax \& K. Hoffm. var. glaber J.J. Sm.

Sulawesi distribution - North

Altitude $-200 \mathrm{~m}$

Collection(s) - Koorders 19628

Drypetes hainanensis Merr.

Sulawesi distribution - North, South

Altitude - 25-50 m

Collection(s) - bb 16980, 24105

Drypetes littoralis (Rob.) Merr.

Sulawesi distribution - North, South-East

Altitude - 0-80 m

Collection(s) - Lam 2840, 2984

Drypetes longifolia (Blume) Pax \& K. Hoffm.

Sulawesi distribution - North, South-East

Altitude - 0-800 m

Collection(s) - Afriastini \& Rohajawati JJA 2883a; bb 5856, 14339, 17568, 29956; De Vogel 2459, 2463; Elbert 3258; Koorders $16265,16270,16271,16272,16273,18510$, 19600,19635

Drypetes macrophylla (Blume) Pax \& K. Hoffm.

Sulawesi distribution - North

Altitude - 150-750 m

Collection(s) - Burley, Partomihardjo \& Rachman 4036, 4074, 4125, 4134

Drypetes maquilingensis (Merr.) Pax \& K. Hoffm.

Sulawesi distribution - Central

Lore Lindu National Park \& environs - present Altitude - 35-50 m

Collection(s) - bb 31856, 31871
Drypetes minahassae (Boerl. \& Koord.) Pax \& K. Hoffm.

Sulawesi distribution - North

Altitude $-0-500 \mathrm{~m}$

Collection(s) - Burley et al. 3575; Koorders $16959,16979,19585,19596$

Drypetes neglecta (Koord.) Pax \& K. Hoffm.

Sulawesi distribution - North, South, SouthEast

Altitude $-0-300 \mathrm{~m}$

Collection(s) - bb 3688, 17182, 17644, 22946; Burley, Partomihardjo \& Rachman 4135, 4144; Cel V 156, V 237; Kjellberg 1257; Meijer 10756; Noerkas 297

Drypetes ovalis (J.J. Sm.) Pax \& K. Hoffm.

Sulawesi distribution - North, South

Altitude $-0-200 \mathrm{~m}$

Collection(s) - bb 24115; Cel I 35, I 35b; Koorders 19449

Drypetes roxburghii Wall.

Sulawesi distribution - Central

Altitude - 0-400 m

Collection(s) - Cel V 184, V 399

\section{Drypetes spec.}

Sulawesi distribution - North, Central, South, South-East

Altitude $-0-300 \mathrm{~m}$

Collection(s) - bb 19406, 19596, 21602, 32509; Cel II 226, II 343, V 224; Church \& Ismail 9; Coode 5946; De Vogel \& Vermeulen 6610; Koorders 19626; Meijer 10752; Prawiroatmodjo \& Soewoko 1709 , 1739, 1772; Whitmore \& Sidiyasa TCW 3417, TCW 3442, TCW 3496, TCW 3526

\section{ENDOSPERMUM}

Endospermum moluccanum (Teijsm. \& Binn.) Becc.

Sulawesi distribution - North

Altitude $-0-50 \mathrm{~m}$

Collection(s) - De Vriese \& Teijsmann 69; Lam 2728 


\section{Endospermum spec.}

Sulawesi distribution - North

Collection(s) - Koorders 16969

\section{EUPHORBIA}

Euphorbia atoto G. Forst.

Sulawesi distribution - South, South-East

Altitude $-5 \mathrm{~m}$

Collection(s) - Elbert 2575, 6064; Hidayat AH 301; Snellius-II 11296A; Teijsmann HB 11990

\section{Euphorbia heterophylla L.}

Sulawesi distribution - North, Central, South Altitude - 0-650 m

Collection(s) - Hidayat AH 275; Johansson 270 ; Noerkas 61

\section{Euphorbia hirta L.}

Sulawesi distribution - North, Central, South Altitude $-1000 \mathrm{~m}$

Collection(s) - Buwalda 3751; De Vogel \& Vermeulen 6851; Eyma 1192, 1744

\section{Euphorbia hypericifolia L.}

Sulawesi distribution - South

Altitude $-1000 \mathrm{~m}$

Collection(s) - Eyma 1209

\section{Euphorbia pilulifera L.}

Sulawesi distribution - North, South, SouthEast

Altitude $-250 \mathrm{~m}$

Collection(s) - Elbert 2860; Koorders 16847; Monod de Froideville 386; Rachmat 93

\section{Euphorbia prostrata Aiton}

Sulawesi distribution - North, Central Lore Lindu National Park \& environs - present Altitude - 0-600 m

Collection(s) - De Vogel \& Vermeulen 7042; Eyma 1722, 1743; Hennipman 5021

Euphorbia rothiana Spreng.

Sulawesi distribution - North, South Altitude - 2100-2800 m

Collection(s) - Bloembergen 3992; Eyma 1346, $1346 \mathrm{~b}$
Euphorbia schumannii Radcl.-Sm.

Sulawesi distribution - Central, South

Altitude $-1000 \mathrm{~m}$

Collection(s) - Monod de Froideville 261; Neth. Ind. For. Service 183; Noerkas 107; Rachmat 970

\section{Euphorbia thymifolia L.}

Sulawesi distribution - North

Collection(s) - Koorders 16922

\section{Euphorbia spec.}

Sulawesi distribution - South

Lore Lindu National Park \& environs - present Altitude - 1700-2200 m

Collection(s) - Meijer 9920

\section{EXCOECARIA}

\section{Excoecaria agallocha L.}

Sulawesi distribution - North, Central, South, South-East

Altitude $-0-200 \mathrm{~m}$

Collection(s) - bb 17193, 21758; Elbert 3273; Grimes 1170; Lam 2449; Noerkas 266, 309, 402

\section{FLUEGGEA}

Flueggea virosa (Roxb. ex Willd.) Voigt

Sulawesi distribution - Central

Lore Lindu National Park \& environs - present Altitude - 10-40 m

Collection(s) - Sidiyasa 1416; Van Balgooy 2989

\section{GALEARIA}

\section{Galearia celebica Koord.}

Sulawesi distribution - North, Central, South Altitude - 0-650 m

Collection(s) - bb 18680; Darnaedi 2277; De Vogel 2547; Johansson 385; Koorders 19593; Meijer 11264

Galearia celebica Koord. var. pubescens Forman

Sulawesi distribution - Central

Lore Lindu National Park \& environs — present 


\author{
Altitude $-650 \mathrm{~m}$ \\ Collection(s) - Johansson 152

\section{GLOCHIDION}

Glochidion acustylum Airy Shaw

Sulawesi distribution - South

Altitude $-500 \mathrm{~m}$

Collection(s) - Van Balgooy 3929

Glochidion album (Blanco) Merr.

Sulawesi distribution - North

Altitude $-150 \mathrm{~m}$

Collection(s) - Burley, Partomihardjo \& Rach$\operatorname{man} 4085$

Glochidion angulatum C.B. Rob.

Sulawesi distribution - North, Central

Collection(s) - Kaudern 475

Glochidion arborescens Blume

Sulawesi distribution - South

Collection(s) - Zollinger 1146, 3538

Glochidion brunneum Hook.f.

Sulawesi distribution - South

Altitude $-400-850 \mathrm{~m}$

Collection(s) - De Vogel 5696, 5828; Yusuf \& Wahyono 165

Glochidion butonicum Airy Shaw

Sulawesi distribution - South-East

Altitude - 0-320 m

Collection(s) - Elbert 2645, 2754

Glochidion dichromum Airy Shaw

Sulawesi distribution - North

Altitude $-600 \mathrm{~m}$

Collection(s) - Koorders 17288

\section{Glochidion dodecapterum Airy Shaw}

Sulawesi distribution - North, Central, South Altitude - 500-1000 m

Collection(s) - Burley, Partomihardjo \& Rachman 3562; Meijer 9954; Van Balgooy 3095

Glochidion eriocarpum Champ.

Sulawesi distribution - South
Altitude $-500 \mathrm{~m}$

Collection(s) - Eyma 535; Van Balgooy 3906

Glochidion formanii Airy Shaw

Sulawesi distribution - North, South

Altitude $-1000 \mathrm{~m}$

Collection(s) - bb 14122; Forman 198; Yoshida 1372

Glochidion glabrum J.J. Sm.

Sulawesi distribution - South

Altitude - $1500 \mathrm{~m}$

Collection(s) - bb 24084

Glochidion gracile Airy Shaw

Sulawesi distribution - Central

Lore Lindu National Park \& environs - present Altitude $-1000 \mathrm{~m}$

Collection(s) - Van Balgooy 3027, 3509

Glochidion hypoleucum (Miq.) Boerl.

Sulawesi distribution - South, South-East

Altitude - $645 \mathrm{~m}$

Collection(s) - Cel IV 123; Elbert 3016; Mamahit 7; Meijer 11079; Teijsmann HB 12584

Glochidion insectum Airy Shaw

Sulawesi distribution - Central

Lore Lindu National Park \& environs - present Altitude $-1000 \mathrm{~m}$

Collection(s) — De Vogel 5085, 5502, 5567

Glochidion insigne J.J. Sm.

Sulawesi distribution - Central

Altitude - $60 \mathrm{~m}$

Collection(s) - Amir 118

Glochidion insigne J.J. Sm. var. glabrescens Airy Shaw

Sulawesi distribution - Central, South

Lore Lindu National Park \& environs - present Altitude - 400-1000 m

Collection(s) - De Vogel 5211, 5248, 5259, 5522,5697

\section{Glochidion lanceilimbum Merr.}

Sulawesi distribution - South, South-East

Altitude $-300 \mathrm{~m}$

Collection(s) - Cel IV 90; Elbert 3447a 


\section{Glochidion littorale Blume}

Sulawesi distribution - South

Altitude $-380 \mathrm{~m}$

Collection(s) - De Vogel 6311; Noerkas 26; Zollinger 3434

Glochidion lucidum Blume

Sulawesi distribution - South-East

Altitude $-20-1100 \mathrm{~m}$

Collection(s) - bb 4189, 21277, 22078

Glochidion lutescens Blume

Sulawesi distribution - South

Altitude - 350-400 m

Collection(s) - De Vogel 5691, 5692; Van Balgooy $3935,3993,3996$

\section{Glochidion luzonense Elmer}

Sulawesi distribution - Central

Lore Lindu National Park \& environs - present Altitude $-250 \mathrm{~m}$

Collection(s) - bb 71

Glochidion molle Blume

Sulawesi distribution - North, Central, SouthEast

Lore Lindu National Park \& environs - present Altitude $-400 \mathrm{~m}$

Collection(s) - bb 5852; Elbert 2677, 2886; Koorders 16866; Meijer 10179

\section{Glochidion moluccanum Blume}

Sulawesi distribution - North

Altitude $-800 \mathrm{~m}$

Collection(s) - Koorders 16864, 16872, 16874, 16876; Meijer 10104

Glochidion moluccanum Blume var. glabrescens Airy Shaw

Sulawesi distribution - Central, South, SouthEast

Altitude - $1000 \mathrm{~m}$

Collection(s) - Elbert 2980; Eyma 371; Noerkas 424; Rachmat 183, 576, 795, 855, 937; Van Balgooy 3534, 3648

Glochidion novoguineense K. Schum.

Sulawesi distribution - South

Collection(s) - Meijer 11215
Glochidion obscurum (Roxb. ex Willd.) Blume

Sulawesi distribution - North, South, SouthEast

Altitude - $1500 \mathrm{~m}$

Collection(s) - bb 5156; Koorders 16877, 16880; McDonald \& Ismail 3870, 4061

Glochidion philippicum (Cav.) C.B. Rob.

Sulawesi distribution - North, Central, South Lore Lindu National Park \& environs - present Altitude - $1050 \mathrm{~m}$

Collection(s) - bb 17971; De Vogel 5591; Koorders 16870; Lam 2730; Meijer 11216; Noerkas 176; Rachmat 31ß, 36

Glochidion pubicapsa Airy Shaw

Sulawesi distribution - South

Altitude - $400 \mathrm{~m}$

Collection(s) - De Vogel 5695

\section{Glochidion rubrum Blume}

Sulawesi distribution - North, Central, South, South-East

Altitude $-400 \mathrm{~m}$

Collection(s) - Boschproefstation 4; Elbert 2540; Hidayat AH 284; Koorders 16868; Lam 3269; Meijer 11309; Rachmat 551; Teijsmann HB 13825

Glochidion stenophyllum Airy Shaw

Sulawesi distribution — South

Altitude $-500 \mathrm{~m}$

Collection(s) - De Vogel 6067

Glochidion sumatranum Miq.

Sulawesi distribution - North, Central, South, South-East

Altitude $-300 \mathrm{~m}$

Collection(s) - bb 17234; Cel II 315, III 45; Elbert 3447; Lam 2529, 2784, 3430; Teijsmann HB 12551

\section{Glochidion ultrabasicola Airy Shaw}

Sulawesi distribution - South

Altitude - $480 \mathrm{~m}$

Collection(s) - Kofman 129; Meijer 11102; Van Balgooy 3703

Glochidion williamsii C.B. Rob.

Sulawesi distribution - South 
Altitude $-1600 \mathrm{~m}$

Collection(s) - bb 29196; Bünnemeyer 11936

Glochidion wonenggau Airy Shaw

Sulawesi distribution - North

Altitude $-1000 \mathrm{~m}$

Collection(s) - bb 14129

Glochidion xerocarpum (O. Schwarz) Airy Shaw

Sulawesi distribution - Central

Collection(s) - Grimes 1219

Glochidion zeylanicum Juss.

Sulawesi distribution - North, South

Altitude $-50 \mathrm{~m}$

Collection(s) - Koorders 16863; Meijer 11071

\section{Glochidion spec.}

Sulawesi distribution - Central

Lore Lindu National Park \& environs - present

Altitude - 1050-1060 m

Collection(s) - Keßler et al. PK 3136, PK 3150

\section{HOMALANTHUS}

\section{Homalanthus populneus (Geiseler) Pax}

Sulawesi distribution - North, Central, South, South-East

Altitude $-0-3000 \mathrm{~m}$

Collection(s) - Achmad 182; Afriastini \& Rohajawati JJA 2967; Amir 189; bb 14127; Bloembergen 4044; Buwalda 3787; Bünnemeyer 11245, 12052, 12336; Church \& Ismail 6; Coode 6151; De Vogel 2610, 5346A, 5408, 5693, 5834, 5933, 7162; De Vogel \& Vermeulen 7291; Elbert 3013, 3262; Eyma 603, 1154, 1614; Hennipman 6015; Johansson 3; Kaudern 425; Kjellberg 508; Koorders 16881, 16882, 16884, 16886; Lam 2527; Meijer 9786, 10748; Rachmat 330, 944; Sands 486, 488, 589; Van Balgooy 3478 , 3631, 3927; Whitmore \& Sidiyasa TCW 3383

\section{HOMONOIA}

\section{Homonoia riparia Lour.}

Sulawesi distribution - North, South Altitude $-250 \mathrm{~m}$
Collection(s) - Afriastini \& Rohajawati JJA 2953; Church \& Ismail 4; Meijer 19684; Teijsmann HB 12442; Whitmore \& Sidiyasa TCW 3478

\section{JATROPHA}

\section{Jatropha curcas L.}

Sulawesi distribution - North, Central, South, South-East

Altitude - $150 \mathrm{~m}$

Collection(s) - Chin 3582; Eyma 4059; Grimes 1042; Prawiroatmodjo \& Maskuri 1271

Jatropha gossypiifolia L.

Sulawesi distribution - Central

Lore Lindu National Park \& environs - present Altitude $-0-100 \mathrm{~m}$

Collection(s) - Meijer 9208, 10170

Jatropha pandurifolia Andr. var. latifolia Pax Sulawesi distribution - South

Collection(s) - Noerkas 62

\section{Jatropha spec.}

Sulawesi distribution - South-East

Altitude $-200 \mathrm{~m}$

Collection(s) - Coode 6105

\section{LASIOCOCCA}

\section{Lasiococca malaccensis Airy Shaw}

Sulawesi distribution - South-East Altitude - $60 \mathrm{~m}$

Collection(s) - bb 26278

\section{MACARANGA}

\section{Macaranga aenigmatica Whitmore}

Sulawesi distribution - Central, South, SouthEast

Altitude - 0-250 m

Collection(s) - bb 21721; Cel II 250, II 340; Grimes 1012; Prawiroatmodjo \& Soewoko 1784

\section{Macaranga allorobinsonii Whitmore}

Sulawesi distribution - North, Central Lore Lindu National Park \& environs - present Altitude - 1000-2050 m 
Collection(s) - bb 17682, 26268; De Vogel 5270

\section{Macaranga carolinensis Volkens}

Sulawesi distribution - Central, South-East

Altitude $-0-300 \mathrm{~m}$

Collection(s) - Elbert 2787; Kaudern 456a, $456 b$

\section{Macaranga carrii L.M. Perry}

Sulawesi distribution - Central

Altitude $-1350 \mathrm{~m}$

Collection(s) - Van Balgooy 3551

Macaranga celebica Koord.

Sulawesi distribution - North, South

Altitude $-0-500 \mathrm{~m}$

Collection(s) - Cel II 217, II 365; De Vogel $6029,6079,6246,6247$; Koorders 16914, 16978

Macaranga conifera (Zoll.) Müll.Arg.

Sulawesi distribution - South

Collection(s) - Cel IV 82

Macaranga cumingii Müll.Arg.

Sulawesi distribution - North

Altitude $-1000 \mathrm{~m}$

Collection(s) - Koorders 16916

Macaranga denticulata (Blume) Müll.Arg.

Sulawesi distribution - South

Collection(s) - Meijer 10905

Macaranga gigantea (Rchb.f. \& Zoll.) Müll.Arg.

Sulawesi distribution - South

Altitude $-100 \mathrm{~m}$

Collection(s) - Cel II 331

Macaranga glaberrima (Hassk.) Airy Shaw

Sulawesi distribution - Central

Lore Lindu National Park \& environs - present

Altitude $-50 \mathrm{~m}$

Collection(s) - De Vogel 5659

Macaranga grandifolia (Blanco) Merr.

Sulawesi distribution - North

Altitude $-200-250 \mathrm{~m}$
Collection(s) - Lam 2859, 2894

Macaranga hispida (Blume) Müll.Arg.

Sulawesi distribution - North, Central, South, South-East

Altitude - 0-1000 m

Collection(s) - Alston 16207; bb 17067; Burley, Partomihardjo \& Rachman 3910, 4116; Cel II 390; De Vogel 2635, 5505, 5709; De Vogel \& Vermeulen 6456, 7051; Elbert 3145; Eyma 1142; Forman 334; Johansson 347; Kjellberg 685; Koorders 16892, 16895, 16901, 16910; Lam 2727; Meijer 9258, 9357, 10935; Milliken 912; Noerkas 337, 358; Prawiroatmodjo \& Maskuri 1152, 1337, 1472, 1555; Tantra 1527; Teijsmann HB 11932; Van Balgooy 3941, 3943; Whitmore \& Sidiyasa TCW 3456

Macaranga involucrata (Roxb.) Baill.

Sulawesi distribution - Central, South, SouthEast

Altitude $-0-400 \mathrm{~m}$

Collection(s) - Coode 5817; De Vogel 5694, 5936; Elbert 2524, 2705, 2706, 2797, 3072, 3304, 3398; Meijer 10806; Teijsmann HB 12123; Widjaja 623

Macaranga loheri Elmer

Sulawesi distribution - South

Altitude $-1300 \mathrm{~m}$

Collection(s) - bb 24164

Macaranga magnifolia L.M. Perry

Sulawesi distribution - South-East

Altitude - 20-250 m

Collection(s) - Prawiroatmodjo \& Soewoko 1965

Macaranga mappa (L.) Müll.Arg.

Sulawesi distribution - North, Central, South, South-East

Altitude $-0-1000 \mathrm{~m}$

Collection(s) - Cel V 219; Koorders 16891, 16898; Lam 3299; Meijer 10936; Prawiroatmodjo \& Soewoko 1721; Van Balgooy 3106; Whitmore \& Sidiyasa TCW 3439

Macaranga minahassae Whitmore

Sulawesi distribution - North

Altitude $-0-700 \mathrm{~m}$ 
Collection(s) - bb 17649; De Vogel \& Vermeulen 6939; Koorders 16897, 16902; Whitmore \& Sidiyasa TCW 3373, TCW 3521

Macaranga rorokae Whitmore

Sulawesi distribution - Central, South

Altitude - 1000-1500 m

Collection(s) - bb 24162, 33065; De Vogel 5050

Macaranga sandsii Whitmore

Sulawesi distribution - South

Altitude $-1650 \mathrm{~m}$

Collection(s) - Sands 368

Macaranga serratifolia Whitmore

Sulawesi distribution - South

Altitude - $1650 \mathrm{~m}$

Collection(s) - Sands 366

Macaranga tanarius (L.) Müll. Arg.

Sulawesi distribution - North, Central, South, South-East

Altitude $-0-950 \mathrm{~m}$

Collection(s) - Amir 83; Bünnemeyer 10584, 10897, 11113; Church \& Ismail 8; Coode 5827; De Vogel 5753; Elbert 2724, 2910, 3376, 3376a, 3436, 3436a; Kaudern 211; Koorders 16900, 19726; Lam 2531; Meijer 10745; Noerkas 113, 188; Rachmat 237, 279, 329, 376a, 717; Teijsmann HB 321, HB 12138, HB 12657, HB 12694, HB 12731, HB 13800; Van Hulstijn 405

Macaranga tanarius (L.) Müll. Arg. var. tanarius

Sulawesi distribution - North, Central, SouthEast

Altitude - 0-650 m

Collection(s) - De Vogel \& Vermeulen 6452, 6458; Elbert 2942; Johansson 343

Macaranga tanarius (L.) Müll. Arg. var. tomentosa (Blume) Müll.Arg.

Sulawesi distribution - North

Altitude - $600 \mathrm{~m}$

Collection(s) - Afriastini \& Rohajawati JJA 2968

Macaranga waturandangii Whitmore

Sulawesi distribution - Central
Lore Lindu National Park \& environs - present Altitude - 1600-1700 m

Collection(s) - bb 20223; Van Balgooy 3384

\section{Macaranga spec.}

Sulawesi distribution - Central, South

Altitude $-0-800 \mathrm{~m}$

Collection(s) - Meijer 9344; Teijsmann HB 13754, HB 13962

\section{MALLOTUS}

Mallotus blumeanus Müll. Arg.

Sulawesi distribution - Central

Lore Lindu National Park \& environs - present Altitude - 650-1000 m

Collection(s) - Johansson 89, 192; Van Balgooy 3055,3427

Mallotus dispar (Blume) Müll.Arg.

Sulawesi distribution - North

Altitude $-500 \mathrm{~m}$

Collection(s) - Koorders 16947, 16970

Mallotus floribundus (Blume) Müll. Arg.

Sulawesi distribution — North, Central, South, South-East

Altitude $-300 \mathrm{~m}$

Collection(s) - Afriastini \& Rohajawati JJA 2930; bb 18084, 25010; Burley, Partomihardjo \& Rachman 4040, 4216; Cel V 293; De Vogel \& Vermeulen 6491, 6936; Forsten 320; Koorders 16903, 16905; Prawiroatmodjo \& Soewoko 2000; Whitmore \& Sidiyasa TCW 3445

Mallotus mollissimus (Geiseler) Airy Shaw

Sulawesi distribution - Central, South

Lore Lindu National Park \& environs - present

Altitude $-1000 \mathrm{~m}$

Collection(s) - Alston 16159; bb 14128, 28281; Cel V 292; Chin 3420; De Vogel 5039, 5920; De Vogel \& Vermeulen 6453, 6680, 6916; Elbert 2693, 2819, 2912, 2943, 2996, 3248, 3416, 3442, 3442a; Forman 254; Johansson 527; Kaudern 64; KeBler et al. PK 3149, PK 3169; Koorders 16907, 16908; Lam 2463, 2645; Meijer 9395, 10112, 10931; Noerkas 355; Prawiroatmodjo \& Maskuri 1266, 1479, 1535; Rachmat 197, 280, 731, 851; Ramlanto \& Fanani 645; Soenarko 310; Teijsmann HB 
14157; Whitmore \& Sidiyasa TCW 3369; Widjaja 623a, 691

Mallotus paniculatus (Lam.) Müll. Arg.

Sulawesi distribution - North, Central, South, South-East

Altitude $-1000 \mathrm{~m}$

Collection(s) - Amir 23; bb 4985, 24977; Eyma 3409; Meijer 11053; Prawiroatmodjo \& Maskuri 1203; Van Balgooy 3515, 3632, 3875; Wardi Wd 67; Yusuf \& Wahyono 67

Mallotus peltatus (Geiseler) Müll.Arg.

Sulawesi distribution - North, Central, South, South-East

Altitude $-755 \mathrm{~m}$

Collection(s) - Afriastini \& Rohajawati JJA 2949; Amir 241; Burley, Partomihardjo \& Rachman 4043, 4121, 4122, 4220, 4239; Coode 6070, 6215; De Vogel 2540; Elbert 3150; Meijer 10727, 10903; Prawiroatmodjo \& Maskuri 1282, 1372, 1403, 1547; Rachmat 800; Teijsmann HB 12570, HB 12687

Mallotus philippensis (Lam.) Müll. Arg.

Sulawesi distribution - Central, South, SouthEast

Lore Lindu National Park \& environs - present

Altitude $-400 \mathrm{~m}$

Collection(s) - bb 18420, 21766; De Vogel 5669; Elbert 2911; Forsten 363; Kjellberg 570; Koorders 16915; Meijer 10236; Noerkas 94a, 268

Mallotus repandus (Willd.) Müll.Arg.

Sulawesi distribution - Central, South, SouthEast

Lore Lindu National Park \& environs - present

Altitude $-600 \mathrm{~m}$

Collection(s) - Elbert 2720; Eyma 3501; Van Balgooy 2980; Van Steenis 10334

Mallotus repandus (Willd.) Müll.Arg. var. genuinus Müll. Arg.

Sulawesi distribution - North

Collection(s) - Forsten 314, 442

Mallotus resinosus (Blanco) Merr.

Sulawesi distribution - North, Central, South

Collection(s) - Kaudern 491, 492; Teijsmann HB 13797, HB 13912
Mallotus tiliifolius (Blume) Müll. Arg.

Sulawesi distribution - North, South

Altitude $-10 \mathrm{~m}$

Collection(s) - Alston 15995; Kaudern 202; Koorders 16932; Lam 3351; Meijer 10116; Yusuf \& Wahyono 35

\section{Mallotus spec.}

Sulawesi distribution - North, Central

Altitude - 80-650 m

Collection(s) - Johansson 286; Ramlanto 114; Whitmore \& Sidiyasa TCW 3524

\section{MANIHOT}

\section{Manihot esculenta Crantz}

Sulawesi distribution - North, South-East

Altitude $-0-600 \mathrm{~m}$

Collection(s) - Elbert 2517, 2737; Forsten 118; Kaudern 6

\section{MELANOLEPIS}

Melanolepis multiglandulosa (Reinw. ex Blume) Rchb.f. \& Zoll.

Sulawesi distribution - North, Central, South, South-East

Altitude $-0-750 \mathrm{~m}$

Collection(s) - Amir 192; bb 17062, 21362; Burley, Partomihardjo \& Rachman 3863; Cel V 289; Coode 6065; De Vogel \& Vermeulen 6465, 6486, 6991; Elbert 2586, 2755, 2848, 3247; Eyma 1590, 3425, 3991; Koorders 16956, 19731, 24071; Lam 2464, 2546; Meijer 9274; Noerkas 160; Prawiroatmodjo \& Maskuri 1074, 1198; Prawiroatmodjo \& Soewoko 1677, 1685; Rachmat 149, 367, 838; Sidiyasa 1396; Wardi Wd 40; Whitmore \& Sidiyasa TCW 3387, TCW 3414

\section{NEOSCORTECHINIA}

Neoscortechinia nicobarica (Hook.f.) Pax \& K. Hoffm.

Sulawesi distribution - North, South, SouthEast

Altitude $-280 \mathrm{~m}$

Collection(s) - bb 19443, 21613,32546, 33110; Cel II 451; Koorders 16974 
Neoscortechinia philippinensis (Merr.) Welzen

Sulawesi distribution - South

Collection(s) - bb 1819, 1871

\section{NEOTREWIA}

Neotrewia cumingii (Müll.Arg.) Pax \& K. Hoffm.

Sulawesi distribution - North, Central, South, South-East

Altitude - 0-1000 m

Collection(s) - Afriastini 2030; bb 14211, 14213; Burley, Partomihardjo \& Rachman 3879, 3893; Burley et al. 3587; De Vogel 5080; De Vogel \& Vermeulen 6492, 7046; Koorders 16929, 16964, 16967, 16968, 16971, 16972; Prawiroatmodjo \& Maskuri 1280; Teijsmann HB 12429

\section{Neotrewia spec.}

Sulawesi distribution - North

Altitude $-0-50 \mathrm{~m}$

Collection(s) - bb 17202

\section{OMPHALEA}

Omphalea bracteata (Blanco) Merr.

Sulawesi distribution - North

Collection(s) - Hose 825; Koorders 16350, 17994, 17997

\section{PACHYSTYLIDIUM}

Pachystylidium hirsutum (Blume) Pax \& K. Hoffm.

Sulawesi distribution - North, South-East Altitude - 0-200 m

Collection(s) - Elbert 3340; Van Balgooy 2974

\section{PHYLLANTHUS}

Phyllanthus amarus Schum. \& Thonn.

Sulawesi distribution - South Lore Lindu National Park \& environs — present Altitude - $1000 \mathrm{~m}$

Collection(s) - Bloembergen 4146

Phyllanthus calycinus Labill.

Sulawesi distribution - South-East
Altitude $-25-150 \mathrm{~m}$

Collection(s) - Elbert 3010

Phyllanthus lamprophyllus Müll.Arg.

Sulawesi distribution - South

Collection(s) - Teijsmann HB 11877

Phyllanthus mindorensis C.B. Rob.

Sulawesi distribution - North, South

Altitude - 0-600 m

Collection(s) - Koorders 16924, 16949, 16954;

Teijsmann HB 11599

Phyllanthus niruri $L$.

Sulawesi distribution - South-East

Altitude - 0-125 m

Collection(s) - Elbert 2531, 2903

Phyllanthus reticulatus Poir.

Sulawesi distribution - Central, South, SouthEast

Altitude - 0-400 m

Collection(s) - De Vogel 5870; Elbert 6518; Hennipman 5838; Metzner 193; Noerkas 37

Phyllanthus reticulatus Poir. forma glaber Müll.Arg.

Sulawesi distribution - South, South-East

Altitude - 0-200 m

Collection(s) - Elbert 3298, 3298a; Teijsmann HB 12338, HB 13851, HB 14153

Phyllanthus reticulatus Poir. forma pubescens J.J. Sm.

Sulawesi distribution - South-East

Altitude - 0-200 m

Collection(s) - Elbert 3358

Phyllanthus samarensis Müll.Arg.

Sulawesi distribution - Central

Lore Lindu National Park \& environs - present Altitude $-10 \mathrm{~m}$

Collection(s) - Van Balgooy 2976

Phyllanthus urinaria L.

Sulawesi distribution - North, Central, South, South-East

Altitude $-0-600 \mathrm{~m}$ 
Collection(s) - Buwalda 3752; Elbert 2928; Eyma 3436, 3522; Kaudern 420; Koorders 18728

Phyllanthus virgatus J.R. Forst. \& G. Forst.

Sulawesi distribution - Central, South, SouthEast

Altitude - 0-125 m

Collection(s) - Elbert 2592, 2679, 2881, 2927; Eyma 3414; Kjellberg 165; Noerkas 71; Van Balgooy 3953

\section{Phyllanthus spec.}

Sulawesi distribution - North, Central, South Lore Lindu National Park \& environs - present Altitude - 0-2200 m

Collection(s) - Alston 16549; Bünnemeyer 12002; De Joncheere 1024; Eyma 3440; Johansson 294; Keßler et al. PK 3154; Teijsmann HB 13902

\section{PIMELODENDRON}

Pimelodendron amboinicum Hassk.

Sulawesi distribution - Central, South, SouthEast, North

Altitude - 0-320 m

Collection(s) - bb 17632, 21337, 29463; Cel II 461, V 171, V 171a; De Vogel \& Vermeulen 6691; Lam 2614, 3043, 3335; Prawiroatmodjo \& Soewoko 2005; Ramlanto \& Fanani 715; Whitmore \& Sidiyasa TCW 3388

\section{RICINUS}

\section{Ricinus communis L.}

Sulawesi distribution - North, Central, South, South-East

Altitude - 0-1450 m

Collection(s) - Bloembergen 4777; Bünnemeyer 11608; Elbert 2703; Eyma 4200; Kaudern 399; Koorders 16931; Wardi Wd 70

\section{SAUROPUS}

Sauropus androgynus (L.) Merr.

Sulawesi distribution - North, Central, South, South-East

Altitude - 0-645 m

Collection(s) - Chin 3597; Elbert 2650, 2689,
2941, 3011, 3041, 3292; Eyma 334; Koorders 16942; Noerkas 196; Rachmat 80, 189, 235; Wardi Wd 56

\section{SPATHIOSTEMON}

\section{Spathiostemon javensis Blume}

Sulawesi distribution - Central, South

Lore Lindu National Park \& environs - present Altitude $-0-300 \mathrm{~m}$

Collection(s) - bb 24130; Cel IV 157; Hennipman 6136; Rachmat 856; Van Balgooy 3589

\section{STROPHIOBLACHIA}

\section{Strophioblachia fimbricalyx Boerl.}

Sulawesi distribution - Central, North

Altitude $-0-40 \mathrm{~m}$

Collection(s) - De Joncheere 1025, 1030; De Vogel 5023; Hennipman 5020; Sidiyasa 1410

\section{SUMBAVIOPSIS}

Sumbaviopsis albicans (Blume) J.J. Sm.

Sulawesi distribution - South

Altitude - $250 \mathrm{~m}$

Collection(s) - Ramlanto 249

\section{SUREGADA}

Suregada glomerulata (Blume) Baill.

Sulawesi distribution - Central, South, SouthEast

Lore Lindu National Park \& environs - present Altitude $-0-400 \mathrm{~m}$

Collection(s) - Elbert 3420; Meijer 10169, 10921; Prawiroatmodjo \& Maskuri 1467

Suregada multiflora (Juss.) Baill.

Sulawesi distribution - South

Altitude - 300-550 m

Collection(s) - Hennipman 6124

\section{TRIADICA}

Triadica cochinchinensis Lour.

Sulawesi distribution - South

Altitude - 0-25 m

Collection(s) - Cel IV 81, IV 81ß, IV 160 


\section{TRIGONOPLEURA}

Trigonopleura malayana Hook.f.

Sulawesi distribution - South

Lore Lindu National Park \& environs — present Altitude - $600 \mathrm{~m}$

Collection(s) - bb 29657

\section{TRIGONOSTEMON}

\section{Trigonostemon spec.}

Sulawesi distribution - South

Collection(s) - Teijsmann HB 11732

\section{FAGACEAE}

\section{CASTANOPSIS}

Castanopsis acuminatissima (Blume) Rheder

Vernacular name(s) - Haleka/Kaha [Napu/ Uma]; jassi [Rante pao]; Koha; Panise; Simimpi; Tjoengaloä

Sulawesi distribution - North, Central, South Altitude - 800-2200 m

Collection(s) - bb 15025, 15089, 15598, 17118, 20198, 23356, 25553, 28266, 29012; Eyma 1515; Koorders 16617; Meijer 9475A; Sands 527; Van Balgooy 3181, 3373, 3400; Zeef 184

\section{LITHOCARPUS}

Lithocarpus caudatifolius (Miq.) Rehder

Sulawesi distribution - North

Collection(s) - Koorders 16633

Lithocarpus celebicus (Miq.) Rehder

Vernacular name(s) - Haleka [Besoa]; Palili

Sulawesi distribution - North, Central, South, South-East

Altitude - 0-1250 m

Collection(s) - bb 19421, 28262, 32801; Cel II 424, IV 152, IV 153, IV 154, V 157; De Vogel 5092, 5093, 5101, 5555; Forman 264; Johansson 200; Koorders 16618, 16622, $16624,16625,16626,16630,16634,16635$, 16636, 16638, 16639, 16670, 16957; Meijer 9259; Prawiroatmodjo \& Soewoko 1697, 1924; Rachmat 1011; Teijsmann HB 5337, HB 12557, HB 12720, HB 14095
Lithocarpus glutinosus (Blume) Soepadmo

Sulawesi distribution - North

Collection(s) - Koorders 16629; Reinwardt 866

Lithocarpus grandifolius (Blume) Mahesw.

Sulawesi distribution - Central

Altitude $-800-1700 \mathrm{~m}$

Collection(s) - bb 20917; Meijer 9295; Van Balgooy 3398

Lithocarpus havilandii (Stapf) Barnett

Sulawesi distribution - Central, South

Lore Lindu National Park \& environs - present Altitude - 400-3000 m

Collection(s) - bb 22858; Bünnemeyer 11935, 12183, 12226; De Vogel 5427, 5483; Eyma 577, 734, 3672; Meijer 9889; Toxopeus 15; Van Balgooy 3349, 3399, 3775

Lithocarpus induta Blume

Sulawesi distribution - Central

Altitude $-1000 \mathrm{~m}$

Collection(s) - Tantra 1505

Lithocarpus menadoensis (Koord.) Soepadmo

Vernacular name(s) - Pasaloli; Woeomeoelo/ Lenghob

Sulawesi distribution - North, South, SouthEast

Altitude - 25-1400 m

Collection(s) - bb 2245, 17542, 24704, 29017, 29473; Cel II 301, II 457, IV 151; Koorders 16632,16642

\section{Lithocarpus spec.}

Vernacular name(s) - Kacuno/Palli [South/ Bug]; Liasa [Soroaka]; Peli [Soroaka]; Polili [Tolaki]

Sulawesi distribution - North, South, SouthEast

Altitude - 75-1700 m

Collection(s) - bb 28289; Coode 6088; De Vogel 2597, 5857, 5862, 5906, 6120, 6208, 6224, 6236, 6363; Hennipman 5755; Meijer 9833, 11437; Van Balgooy 3974; Whitmore \& Sidiyasa TCW 3520, TCW 3540; Yoshida 1382 
TRIGONOBALANUS

Trigonobalanus verticillata Forman

Sulawesi distribution - South

Altitude - 15-1100 m

Collection(s) - bb 20206, 22621, 33063

\section{FLACOURTIACEAE}

CASEARIA

\section{Casearia grewiaefolia Vent.}

Vernacular name(s) - Karikis lawanan; Karikis sela; Soensoean

Sulawesi distribution - North, Central, SouthEast

Altitude - 20-450 m

Collection(s) — bb 29475, 31486; Burley, Partomihardjo \& Rachman 3883; Koorders 18797 , 18799, 18800; Prawiroatmodjo \& Soewoko 1674

Casearia grewiaefolia Vent. var. gelonioides (Blume) Sleumer

Vernacular name(s) - Donga-donga; Lemboa Sulawesi distribution - North, Central, South, South-East

Altitude - 10-1000 m

Collection(s) - bb 21594, 31919; Béguin 201; Elbert 3469; Kjellberg 585; Koorders 18796, 18798; Teijsmann HB 11868, HB 12089, HB 13815; Van Balgooy 3105

Casearia spec.

Sulawesi distribution - Central, South Altitude - 300-1800 m

Collection(s) - De Vogel 5442, 6292, 6399

\section{ERYTHROSPERMUM}

Erythrospermum candidum Becc.

Sulawesi distribution - South

Collection(s) - Teijsmann HB 11869

\section{FLACOURTIA}

Flacourtia indica (Burm.f.) Merr.

Sulawesi distribution - South

Collection(s) - Teijsmann HB 14140
Flacourtia inermis Roxb.

Vernacular name(s) - Tomi-tomi

Sulawesi distribution - North

Altitude $-10 \mathrm{~m}$

Collection(s) - Koorders 16260; Vorderman 7

Flacourtia rukam Zoll. \& Moritzi

Sulawesi distribution - South

Altitude $-400 \mathrm{~m}$

Collection(s) - De Vogel 5923

Flacourtia zippelii Slooten

Vernacular name(s) - Boenaroh

Sulawesi distribution - North, South

Altitude - 75-500 m

Collection(s) - bb 25540; Koorders 16269; Lam 3174; Meijer 10848

\section{HOMALIUM}

Homalium caryophyllaceum (Zoll. \& Moritzi) Benth.

Sulawesi distribution - South

Altitude $-2 \mathrm{~m}$

Collection(s) - bb 19691

\section{Homalium celebicum Koord.}

Vernacular name(s) - Kari(s)kis/Kaikis; Karikis meka; Karikis rinlek; Karingis; Kombolili

Sulawesi distribution - North, Central, South Altitude - 4-550 m

Collection(s) - bb 8452, 31882; Koorders 18803, 18811, 18812, 18813, 18815; Lam 2431; Teijsmann HB 11830, HB 12296

Homalium foetidum (Roxb.) Benth.

Vernacular name(s) - Gia; Kajoe besi [intaloen]; Kajoe bessi; Kolaka molaka/Longor; Tohasik

Sulawesi distribution - North, South, SouthEast

Altitude - 10-350 m

Collection(s) - bb 4992, 17495, 18014, 22988, 23094, 23291, 23293, 23295, 32462, 32602, 33121; Cel II 386; Ham L 1; Koorders 18804, $18805,18806,18807,18808,18810,18814$

Homalium minahassae Koord.

Sulawesi distribution - North

Altitude $-50 \mathrm{~m}$

Collection(s) - Koorders 19455 


\section{Homalium spec.}

Sulawesi distribution - North

Collection(s) - De Vriese \& Teijsmann 82; Koorders 16259, 16262

\section{HYDNOCARPUS}

Hydnocarpus heterophylla Blume subsp. philippinensis Sleumer

Sulawesi distribution - Central

Collection(s) - bb 31864

Hydnocarpus sumatrana (Miq.) Koord.

Sulawesi distribution - North, South-East

Altitude - 20-250 m

Collection(s) - Burley, Partomihardjo \& Rachman 4221; De Vogel 2580; Koorders 18795, 19631; Prawiroatmodjo \& Maskuri 1090, 1427; Prawiroatmodjo \& Soewoko 1870

Hydnocarpus spec.

Sulawesi distribution - North

Altitude - 350-750 m

Collection(s) - Burley, Partomihardjo \& Rachman 3566

ITOA

Itoa stapfii (Koord.) Sleumer

Sulawesi distribution - North, Central, South Altitude - 500-1000 m

Collection(s) - bb 26293; Koorders 16888; Van Balgooy 3092

OSMELIA

Osmelia philippina (Turcz.) Benth

Vernacular name(s) - Madaoesiep

Sulawesi distribution - North, South, SouthEast

Altitude - 50-1000 m

Collection(s) - bb 14137; Forman 228, 242; Koorders 18794; Meijer 9990; Prawiroatmodjo \& Maskuri 1283

\section{PANGIUM}

Pangium edule Reinw.

Vernacular name(s) - Pangi
Sulawesi distribution - North, Central, South, South-East

Altitude $-20-500 \mathrm{~m}$

Collection(s) - bb 24483, 28277; Cel V 126, V 338; Coode 5937, 6216; De Vogel 2543; Koorders 16268, 19764; Prawiroatmodjo \& Soewoko 1895; Whitmore \& Sidiyasa TCW 3457, TCW 3458

\section{RYPAROSA}

Ryparosa javanica (Blume) Kurz

Sulawesi distribution - South

Lore Lindu National Park \& environs - present Altitude $-50 \mathrm{~m}$

Collection(s) - Meijer 10788

SCOLOPIA

\section{Scolopia crenata Clos}

Sulawesi distribution - South

Collection(s) - Meijer 10850

Scolopia luzonensis (Presl) Warb.

Sulawesi distribution - South, South-East

Altitude - 0-497 m

Collection(s) - Elbert 3120, 3230, 3315; Teijsmann HB 11808, HB 12207, HB 12278, HB 12315, HB 12330

Scolopia spinosa (Roxb.) Warb.

Sulawesi distribution - South-East

Altitude - 700-900 m

Collection(s) - McDonald \& Ismail 4174

\section{TRICHADENIA}

Trichadenia philippinensis Merr.

Sulawesi distribution - South-East

Altitude - $15 \mathrm{~m}$

Collection(s) - bb 21608

\section{XYLOSMA}

Xylosma luzonensis (Presl) Clos

Sulawesi distribution - South-East

Altitude - 0-75 m

Collection(s) - Elbert 2663 


\section{GNETACEAE}

\section{GNETUM}

\section{Gnetum cuspidatum Blume}

Vernacular name(s) - bankino badja [Saloean] Sulawesi distribution - North, Central, South Altitude - 170-1150 m

Collection(s) - Eyma 3803; Hennipman 6057; Koorders 17213; Lam 2760, 2909, 3317; Ramlanto 170

\section{Gnetum gnemon L.}

Vernacular name(s) - Behu [Uma]; Saede; soa Sulawesi distribution - North, Central, South, South-East

Altitude - $900 \mathrm{~m}$

Collection(s) - bb 1902, 5869, 17968, 21702, 24980; De Vogel 5624; Elbert 2940, 3134, 3457, 3492; Johansson 324; Koorders 17207 , 17217; Lam 2545, 3034, 3113, 3275; Meijer 11249; Prawiroatmodjo \& Maskuri 1441, 1508; Prawiroatmodjo \& Soewoko 1765; Ramlanto \& Fanani 733; Teijsmann HB 12519; Whitmore \& Sidiyasa 3396

\section{Gnetum gnemon L. var. gnemon}

Vernacular name(s) - Kai-Kai

Sulawesi distribution - North, South-East

Altitude - 200-300 m

Collection(s) - Koorders 17208; Meijer 10062; Prawiroatmodjo \& Soewoko 1932; Rachmat 159

\section{Gnetum latifolium Blume}

Sulawesi distribution - North, South

Altitude $-750 \mathrm{~m}$

Collection(s) - Kaudern 5, 172; Meijer 10847, 10957; Prawiroatmodjo \& Soewoko 1750

\section{Gnetum latifolium Blume var. funiculare} (Blume) Miq.

Sulawesi distribution - South-East

Altitude $-50 \mathrm{~m}$

Collection(s) - Prawiroatmodjo \& Maskuri 1330

\section{Gnetum latifolium Blume var. latifolium}

Sulawesi distribution - North, South, SouthEast

Altitude $-20-1500 \mathrm{~m}$
Collection(s) - De Vogel 2542; Forsten 347; Hennipman 5825; Meijer 9602, 10920; Prawiroatmodjo \& Soewoko 1825; Teijsmann HB 12568; Van Balgooy 3679, 3807

\section{Gnetum spec.}

Sulawesi distribution - North, Central, South Altitude - $420 \mathrm{~m}$

Collection(s) - Coode 5940; De Vogel 5761, 6230; De Vogel \& Vermeulen 6496, 6552, 6732, 6743; De Vogel et al. 5761; Whitmore \& Sidiyasa 3394

\section{GOODENIACEAE}

\section{SCAEVOLA}

\section{Scaevola micrantha Presl}

Vernacular name(s) - Panimburan'a

Sulawesi distribution - North

Altitude $-300 \mathrm{~m}$

Collection(s) - Lam 3266

Scaevola oppositifolia R.Br.

Sulawesi distribution - North, Central, South, South-East

Altitude $-125-2200 \mathrm{~m}$

Collection(s) - Alston 15812; Bünnemeyer 11040, 11411, 11994; De Vogel 5971; Elbert 3019; Eyma 1450, 4024; Kaudern 518; Koorders 18380, 18381, 18386; Meijer 11310, 11507; Prawiroatmodjo \& Maskuri 1111; Rachmat 525; Van Balgooy 3301, 3628; Yoshida 1012

\section{Scaevola sericea Vahl}

Vernacular name(s) - Panemboeran

Sulawesi distribution - North, Central, South, South-East

Altitude $-0-100 \mathrm{~m}$

Collection(s) - Alston 16014; Elbert 2564, 3239; Eyma 3583; Grimes 1183; Koorders 17219, 17220; Lam 3375, 3425; Rachmat 689, 773; Snellius-II 11299A, 11593A 


\section{GUTTIFERAE}

\section{CRATOXYLON}

\section{Cratoxylon spec.}

Vernacular name(s) - Peringan/Walahopa [Palopo/Kendari]; Uing [Kotamobagu]

Sulawesi distribution - North, South, SouthEast

Altitude - 20-600 m

Collection(s) - De Vogel 5715, 5879, 6019, 6181, 6693; De Vogel \& Vermeulen 6886, 6914A; Hennipman 5827

\section{GARCINIA}

Garcinia balica Miq.

Sulawesi distribution - South

Collection(s) - Meijer 11153; Noerkas 425

Garcinia celebica L.

Sulawesi distribution - Central, South, SouthEast

Altitude - 15-300 m

Collection(s) - bb 32514; Burghardt 5855; Cel II 112; Ramlanto \& Fanani 721; Teijsmann HB 11843, HB 12392

\section{Garcinia cornea L.}

Vernacular name(s) - Panggamuil'a

Sulawesi distribution - North

Altitude $-300 \mathrm{~m}$

Collection(s) — Lam 3309

\section{Garcinia daedalanthera Pierre}

Vernacular name(s) - Mabagoestang

Sulawesi distribution - North, Central

Altitude - 50-600 m

Collection(s) - bb 13509; De Vriese 138; Koorders 17312, 17314, 17315, 17321, 17324, 17337, 17339; Ramlanto \& Fanani 651

Garcinia dulcis (Roxb.) Kurz

Vernacular name(s) - Boeno Soewai; Mamoendoeng

Sulawesi distribution - North, South, SouthEast

Altitude - 0-600 m

Collection(s) - bb 6039, 13756, 17569, 17966, 21761; Cel II 435; De Vogel 2455; Elbert
3244, 3361, 3362; Kjellberg 630; Koorders 17319, 17322, 17338; Meijer 10817; Teijsmann HB 12547, HB 12730

\section{Garcinia lateriflora Blume}

Sulawesi distribution - North, Central

Altitude - 200-750 m

Collection(s) - Burley, Partomihardjo \& Rachman 3518, 3523; Ramlanto \& Fanani 719

Garcinia maluensis Lauterb.

Sulawesi distribution - North

Altitude - 350-750 m

Collection(s) - Burley, Partomihardjo \& Rachman 4015

\section{Garcinia minahassensis Pierre}

Sulawesi distribution - North, Central

Altitude - $500 \mathrm{~m}$

Collection(s) - Kaudern 389; Koorders 17310 , 17326

Garcinia morella Boerl.

Sulawesi distribution - South

Collection(s) — Meijer 10919

Garcinia parviflora (Miq.) Miq.

Sulawesi distribution - North, South

Altitude - 50-600 m

Collection(s) - Koorders 17320, 17328; Ramlanto 186

\section{Garcinia picorhiza Miq.}

Sulawesi distribution - North, South

Altitude - 300-500 m

Collection(s) - Koorders 17323, 17325, 17332, 17333; Teijsmann HB 14171

\section{Garcinia rhizophorides Elmer}

Vernacular name(s) - burit'a

Sulawesi distribution - North

Altitude $-500 \mathrm{~m}$

Collection(s) - Lam 3288

\section{Garcinia tetrandra Pierre}

Sulawesi distribution - North, South-East

Altitude - 0-100 m

Collection(s) - De Vogel 2442, 2535; Prawiroatmodjo \& Maskuri 1088 


\section{Garcinia treubii Pierre}

Sulawesi distribution - South

Collection(s) - Meijer 10774

\section{Garcinia spec.}

Vernacular name(s) — kordies [Saloean]; Mandoela; Maro [Besoa]; Mire [Timampu]; Mire Ilandora; Mire Pae; Mire sinari; Mire soewai; Mire toewai

Sulawesi distribution - North, Central, South, South-East

Altitude - 0-1600 m

Collection(s) - Amir 172; bb 19600, 20862, 21730, 23911, 25534, 25536, 26016, 26294, 28291; Burley, Partomihardjo \& Rachman $3602,3718,3729,3848,3986,4005,4020$; Cel II 359, II 417, II 418, II 429, II 443, III 108, V 296; Coode 5943, 5951, 6018, 6019, 6050,6282 ; De Vogel 2456, 2538, 5489, $6189,6290,6354$; De Vogel \& Vermeulen 6494, 6501, 6509, 6564, 6613, 6638; Elbert $3357,3384,3402$, 3403; Eyma 3804; Forman 306; Hennipman 6076; Johansson 290; Keßler et al. PK 3133; Koorders 17308, $17311,17330,17331,17334,17335,17336$, 18385; Kostermans \& Soegeng Reksodihardjo 2; Lam 2826; Mamahit 16; Meijer $11095,11244,11283,11303$; Prawiroatmodjo \& Soewoko $1667,1711,1740,1745$, 1746, 1828, 1831, 1931; Rachmat 393; Ramlanto 245; Ramlanto \& Fanani 480; Teijsmann HB 11705; Van Balgooy 3109 , $3115,3769,3872$, 3877, 4011; Van Vuuren 230; Whitmore \& Sidiyasa TCW 3395, TCW 3438, TCW 3453, TCW 3499, TCW 3501

\section{MESUA}

\section{Mesua spec.}

Vernacular name(s) - Moro wetai; Wetai koeni; Wetai naoe-naoe; Wetai singko

Sulawesi distribution - Central, South, SouthEast

Altitude $-250-300 \mathrm{~m}$

Collection(s) - Cel II 223, II 410, II 425, II 442 , II 443, V 210

\section{HERNANDIACEAE}

\section{HERNANDIA}

\section{Hernandia nymphaeifolia (Presl) Kubitzki}

Sulawesi distribution - North, Central, South Lore Lindu National Park \& environs - present Altitude - 0-320 m

Collection(s) - Elbert 2727; Eyma 3725; Koorders 17418, 17419, 17420; Rachmat 764; Van Balgooy 3588

\section{Hernandia ovigera L.}

Vernacular name(s) - Lilok'a oeroene Sulawesi distribution - North, Central Altitude $-30-500 \mathrm{~m}$

Collection(s) - bb 28233; Koorders 16701, 17414, 17415, 17417; Lam 2839

\section{HIMANTANDRACEAE}

\section{GALBULIMINA}

Galbulimima belgraveana (F. Muell.) Sprague

Sulawesi distribution - Central

Altitude $-500-1500 \mathrm{~m}$

Collection(s) - Meijer 10003; Van Balgooy $3012,3541,3962$

\section{ICACINACEAE}

\section{APODYTES}

Apodytes dimidiata E. Mey. ex Arn.

Sulawesi distribution - Central

Collection(s) - Lack \& Grimes 1676

\section{CITRONELLA}

Citronella suaveolens (Blume) Howard

Vernacular name(s) - Baloelalembo; Kajoe sokkah; Pano-pano

Sulawesi distribution - North, South

Altitude - 700-1600 m

Collection(s) - bb 20434; Cel II 305, II 348; Koorders 19595; Teijsmann HB 13779, HB 13985, HB 14118 


\section{GOMPHANDRA}

\section{Gomphandra mappioides Valeton}

Vernacular name(s) - Lowa poete; Sowa poete; Taloeha

Sulawesi distribution - North, South

Altitude - 0-1000 m

Collection(s) - bb 18683; Burley, Partomihardjo \& Rachman 3540, 3828; Cel V 197, V 230; De Vogel 2670; Hennipman 6084, 6085; Koorders 17481, 18349, 18350, 18351, $18352,18353,18354,18355,18356,18357$, 18362; Ramlanto 213; Teijsmann HB 11742 , HB 11973, HB 12841, HB 13783, HB 14033; Van Vuuren 445

\section{Gomphandra spec.}

Sulawesi distribution - South

Collection(s) - Meijer 10984

\section{GONOCARYUM}

Gonocaryum calleryanum (Baill.) Becc.

Sulawesi distribution - North

Altitude $-200 \mathrm{~m}$

Collection(s) - Church \& Ismail 25

Gonocaryum littorale (Blume) Sleumer

Vernacular name(s) - Baloela; Karo

Sulawesi distribution - Central, South, SouthEast

Altitude - 0-250 m

Collection(s) - bb 6649, 32324; Cel II 489, IV 184; Prawiroatmodjo \& Maskuri 1503; Prawiroatmodjo \& Soewoko 1678

\section{Gonocaryum spec.}

Sulawesi distribution - South-East

Altitude $-100-200 \mathrm{~m}$

Collection(s) - Prawiroatmodjo \& Soewoko 1916

\section{IODES}

Iodes cirrhosa Turcz.

Vernacular name(s) - Bindalahe; Laweha

Sulawesi distribution - North, Central, South, South-East

Altitude - 50-1000 m

Collection(s) - Church \& Ismail 22; De Vogel
\& Vermeulen 6888; Forsten 7, 14, 24, 95 , 385; Kaudern 50; Koorders 18343, 18345, 18346, 18348; Meijer 9398; Prawiroatmodjo \& Maskuri 1075; Teijsmann HB 12624; Van Balgooy 2959; Whitmore \& Sidiyasa TCW 3487

Iodes philippinensis Merr.

Sulawesi distribution - North

Altitude - 50-130 m

Collection(s) - Lam 2807, 3211

\section{MEDUSANTHERA}

Medusanthera laxiflora (Miers) Howard

Sulawesi distribution - Central, South, SouthEast

Altitude $-10-250 \mathrm{~m}$

Collection(s) - bb 21359, 21597; Cel V 180

MIQUELIA

\section{Miquelia celebica Blume}

Sulawesi distribution - North, Central, SouthEast

Lore Lindu National Park \& environs - present Altitude - 0-650 m

Collection(s) - Elbert 2818; Johansson 383; Koorders 19594; Kruiff 23

\section{PHYTOCRENE}

\section{Phytocrene hirsuta Blume}

Sulawesi distribution - North, Central, South, South-East

Altitude - 0-650 m

Collection(s) - Burley, Partomihardjo \& Rachman 4010, 4066; Elbert 2997; Johansson 296; Kjellberg 311; Koorders 18361, 19411; Meijer 10818; Teijsmann HB 11823, HB 11838, HB 12258, HB 12714, HB 13690, HB 14055; Whitmore \& Sidiyasa TCW 3443 , TCW 3543

\section{Phytocrene macrophylla Blume}

Sulawesi distribution - North, Central, SouthEast

Lore Lindu National Park \& environs - present Altitude - 20-650 m

Collection(s) - De Vogel 2640; Johansson 390; Prawiroatmodjo \& Soewoko 1792 
Phytocrene macrophylla (Blume) Blume var. dasycarpa (Miq.) Sleumer

Vernacular name(s) - Tali-utanarteh/Samad Sulawesi distribution - North, Central, South Lore Lindu National Park \& environs - - present Altitude - 0-1000 m

Collection(s) - Alston 16054; De Vogel 5088; De Vogel \& Vermeulen 6700, 6723; De Vriese \& Teijsmann 22; Koorders 17992, 17996 , 18359; Reinwardt 12; Teijsmann HB 5211, HB 5271, HB 5791; Van Balgooy 3045, 4024

\section{Phytocrene spec.}

Sulawesi distribution - Central, South-East Altitude - 90-200 m

Collection(s) - Coode 6059, 6103, 6104

\section{STEMONURUS}

Stemonurus celebicus Valeton

Sulawesi distribution - North

Altitude - 0-500 m

Collection(s) - Koorders 18363, 19572

\section{IXONANTHACEAE}

\section{IXONANTHES}

\section{Ixonanthes petiolaris Blume}

Vernacular name(s) - Loera poete

Sulawesi distribution - South

Collection(s) - Cel III 5

\section{Ixonanthes reticulata Jack}

Vernacular name(s) - Loera

Sulawesi distribution - South

Altitude $-200-420 \mathrm{~m}$

Collection(s) - Cel II 357; De Vogel 6222

\section{Ixonanthes spec.}

Sulawesi distribution - South, South-East Altitude - 15-600 m

Collection(s) - bb 20902, 21609, 23266, 25564, 29659

\section{JUGLANDACEAE}

\section{ENGELHARDIA}

\section{Engelhardia rigida Blume}

Sulawesi distribution - Central, South, SouthEast

Lore Lindu National Park \& environs - present

Altitude - 300-1600 m

Collection(s) - bb 28224; Coode 6192; De Vogel 6353; Meijer 9254, 9404; Tantra 1558; Teijsmann HB 14144; Van Balgooy 3152

Engelhardia serrata Blume

Sulawesi distribution - South

Collection(s) - bb 19555, 20866, 23354, 24085; Kaudern 516; Meijer 9916

\section{LAURACEAE}

\section{ACTINODAPHNE}

Actinodaphne angustifolia Nees

Sulawesi distribution - South

Altitude - $450 \mathrm{~m}$

Collection(s) - Yusuf \& Wahyono 10

Actinodaphne glomerata Nees

Sulawesi distribution - South

Altitude $-1300 \mathrm{~m}$

Collection(s) - bb 24156

Actinodaphne intermedia (Elmer) Kosterm.

Vernacular name(s) - Tahurimpat'a

Sulawesi distribution - North, South

Altitude - 200-900 m

Collection(s) - Koorders 17453, 17468, 17510, 17511; Lam 3267; Teijsmann HB 11758, HB 11762

\section{Actinodaphne spec.}

Vernacular name(s) - Tahurimpat'a; Waraoeloe Sulawesi distribution - North, Central, South, South-East

Altitude - 20-3440 m

Collection(s) - Cel V 177; Coode 6080, 6277; De Vogel 5285; Elbert 3142; Eyma 775, 3627; Forman 398; Johansson 336; Koorders 17489,17502 ; Kostermans \& Soegeng Reksodihardjo 3; Lam 2694; Meijer 9123, 9717, 
10775, 10843; Prawiroatmodjo \& Soewoko 1631, 1872, 1917; Ramlanto \& Fanani 678; Teijsmann HB 13592, HB 14028; Van Balgooy 3549,4022

\section{ALSEODAPHNE}

Alseodaphne lancilimba Kosterm.

Sulawesi distribution - South

Collection(s) - Teijsmann HB 11856

\section{BEILSCHMIEDIA}

Beilschmiedia gemmiflora (Blume) Kosterm.

Sulawesi distribution - South, South-East

Collection(s) - bb 21091; Cel I 95, V 130, V 195, V 195a

\section{Beilschmiedia gigantocarpa Kosterm.}

Sulawesi distribution - South

Altitude $-250 \mathrm{~m}$

Collection(s) - Afriastini 2077; bb 23921; Cel IV 98, IV 110, IV 185, IV 186, IV 187, IV 188; Van Balgooy 3924

\section{Beilschmiedia spec.}

Sulawesi distribution - Central, South, SouthEast

Lore Lindu National Park \& environs - present

Collection(s) - Coode 6094; Johansson 404; Meijer 9957; Yusuf \& Wahyono 119

\section{CASSYTHA}

\section{Cassytha filiformis L.}

Sulawesi distribution - North, Central, South, South-East

Lore Lindu National Park \& environs - present Altitude - 0-600 m

Collection(s) - De Vogel 5025; Elbert 2522; Eyma 367, 4011; Koorders 17398, 17399; Lam 3241; Van Balgooy 4045

\section{CINNAMOMUM}

Cinnamomum burmanni Nees ex Blume

Sulawesi distribution - North, Central, South Altitude $-0-1000 \mathrm{~m}$

Collection(s) - bb 31909; Teijsmann HB 5306, HB 13951

\section{Cinnamomum celebicum Miq.}

Vernacular name(s) - Balisu rintek; Lawang

Sulawesi distribution - North, Central, South, South-East

Altitude - 0-2050 m

Collection(s) - bb 5555, 24159, 26273; Cel V 321; Elbert 3111; Eyma 4096; Koorders 17401; Musser 295

Cinnamomum pilosum Cammerloher

Sulawesi distribution - South

Altitude - 0-1600 m

Collection(s) - bb 20219; Teijsmann HB 13753, HB 14192

Cinnamomum polderi Kosterm.

Sulawesi distribution - North, South

Altitude - 700-1600 m

Collection(s) - Koorders 17404, 17408; Meijer 9429

Cinnamomum porrectum (Roxb.) Kosterm.

Sulawesi distribution - Central, South

Lore Lindu National Park \& environs - present Altitude - 250-800 m

Collection(s) - bb 24492, 28244; Cel V 279; Meijer 9394

\section{Cinnamomum subaveniopsis Kosterm.}

Vernacular name(s) - Ikondongi awoe

Sulawesi distribution - North, South

Altitude - 0-1250 m

Collection(s) - bb 28264; Cel IV 94

Cinnamomum subavenium Miq.

Sulawesi distribution - South

Collection(s) - Meijer 11509

Cinnamomum sulavesianum Kosterm.

Sulawesi distribution - South

Altitude - 0-200 m

Collection(s) - Cel 465, II 148, III 57

Cinnamomum trichophyllum Quinn \& Merr.

Vernacular name(s) - Aringa

Sulawesi distribution - North, Central, South Altitude - 250-1100 m

Collection(s) - Koorders 17402, 17405; Lam 2903; Meijer 9759; Musser 909, S 54a 


\section{Cinnamomum spec.}

Sulawesi distribution - South

Altitude $-400 \mathrm{~m}$

Collection(s) - De Vogel 6201

\section{CRYPTOCARYA}

\section{Cryptocarya ampla Merr.}

Sulawesi distribution - South

Altitude $-50 \mathrm{~m}$

Collection(s) - bb 24499

\section{Cryptocarya cagayanensis Merr.}

Sulawesi distribution - North, South-East

Altitude $-70 \mathrm{~m}$

Collection(s) - bb 24986, 31826; Koorders 17480

Cryptocarya celebica (Koord.) Kosterm.

Vernacular name(s) - Monaladoti

Sulawesi distribution - North

Altitude $-200-350 \mathrm{~m}$

Collection(s) - bb 17177; Burley, Partomihardjo \& Rachman 3551; Church \& Ismail 14; Koorders 16873, 19627

Cryptocarya crassinervia Miq.

Sulawesi distribution - South

Altitude $-850 \mathrm{~m}$

Collection(s) - Yusuf \& Wahyono 52

Cryptocarya crassinerviopsis Kosterm.

Sulawesi distribution - Central, South

Altitude - 1000-1200 m

Collection(s) - De Vogel 5496; Meijer 9725; Van Balgooy 3537

\section{Cryptocarya ferrea Blume}

Sulawesi distribution - North, South

Altitude $-25-1000 \mathrm{~m}$

Collection(s) - bb 18020; Koorders 17447, $17456,17479,17506,17508$

\section{Cryptocarya glauca Merr.}

Vernacular name(s) - Tiempallang katji

Sulawesi distribution - North

Altitude - 50-600 m

Collection(s) - Koorders 17455, 17469, 17483, 17509; Teijsmann HB 12556, HB 12722

\section{Cryptocarya laevigata Blume}

Sulawesi distribution - North

Altitude - 500-1000 m

Collection(s) - De Vogel 2628; Koorders 17403,17522

\section{Cryptocarya lancifolia Kosterm.}

Sulawesi distribution - South

Altitude $-150 \mathrm{~m}$

Collection(s) - Van Balgooy 3900

Cryptocarya microcos Kosterm.

Sulawesi distribution - South

Altitude - 25-100 m

Collection(s) - Cel 342, IV 122

Cryptocarya nitidissima Kosterm.

Sulawesi distribution - South

Collection(s) - bb 12662

\section{Cryptocarya palawanensis Merr.}

Vernacular name(s) - Ikondonge molaba; Ikondongimolaba; Tenggera

Sulawesi distribution - South

Altitude - $200 \mathrm{~m}$

Collection(s) - Cel V 127, V 202

\section{Cryptocarya subvelutina Elmer}

Vernacular name(s) 一 Lekak; Wiakan

Sulawesi distribution - North

Altitude $-150-800 \mathrm{~m}$

Collection(s) - bb 12658, 14334, 17059; De Vriese \& Teijsmann 62, 66; Koorders 17451

\section{Cryptocarya sulavesiana Kosterm.}

Sulawesi distribution - South

Collection(s) - Cel 346

\section{Cryptocarya triplinervis R.Br.}

Vernacular name(s) - Garete borong

Sulawesi distribution - South

Altitude $-200 \mathrm{~m}$

Collection(s) - Cel I 89

\section{Cryptocarya spec.}

Vernacular name(s) - Anak Ntawine [Uma]; Kuma [Mekongkas]; Kure [Besoa]

Sulawesi distribution - North, Central, South, South-East

Altitude $-20-2200 \mathrm{~m}$ 
Collection(s) - bb 24077; Coode 6149; De Vogel 2637, 5257, 5552, 5812, 5830, 6219; De Vogel \& Vermeulen 6662; Johansson 330; Meijer 9431; Prawiroatmodjo \& Soewoko 1770, 1943; Van Balgooy 3146, 3360, 3902; Widjaja 801

\section{DEHAASIA}

\section{Dehaasia caesia Blume}

Sulawesi distribution - Central, South

Altitude $-25 \mathrm{~m}$

Collection(s) - bb 31852; Meijer 10912

Dehaasia cairocan (Vidal) Allen

Sulawesi distribution - Central

Altitude $-75 \mathrm{~m}$

Collection(s) - bb 31900

Dehaasia celebica Kosterm.

Sulawesi distribution - North, Central, South, South-East

Altitude - 70-430 m

Collection(s) - bb 18416, 21751, 24983; Cel II 150, II 476; De Vogel 6258; Elbert 3281; Lam 3314

\section{Dehaasia spec.}

Sulawesi distribution - South, South-East

Altitude - 200-1000 m

Collection(s) - bb 22957; Coode 6095; Meijer 9547

\section{ENDIANDRA}

Endiandra celebica Kosterm.

Sulawesi distribution - South, South-East

Altitude - 0-250 m

Collection(s) - Cel 133, IV 180; Prawiroatmodjo \& Soewoko 1706

\section{Endiandra laxiflora Merr.}

Sulawesi distribution - North, South

Altitude - 40-500 m

Collection(s) - bb 17205; Burley, Partomihardjo \& Rachman 3753; De Vogel 6074

Endiandra rubescens (Blume) Miq.

Sulawesi distribution - South-East

Altitude $-50 \mathrm{~m}$
Collection(s) - Prawiroatmodjo \& Maskuri 1277,1293

Endiandra scrobiculata Kosterm.

Sulawesi distribution - South

Collection(s) - bb 1864

Endiandra sulavesiana Kosterm.

Sulawesi distribution - North

Altitude $-0-300 \mathrm{~m}$

Collection(s) - Burley, Partomihardjo \& Rachman 3588; Koorders 16644, 17440, 17526, 17780; Kostermans 11075

Endiandra velutina Kosterm.

Sulawesi distribution - South

Altitude - 350-1650 m

Collection(s) - Cel V 221; Sands 369

Endiandra spec.

Sulawesi distribution - Central, South

Altitude - 0-1000 m

Collection(s) - Burley et al. 3711, 3804, 4000; Kofman 140; Van Balgooy 3131

\section{LINDERA}

\section{Lindera apoensis Elmer}

Sulawesi distribution - North

Altitude - $700 \mathrm{~m}$

Collection(s) - Burley, Partomihardjo \& Rachman 4028

Lindera cuspidata (Blume) Boerl.

Vernacular name(s) - Boekang Breboe; Raripungi mewakan

Sulawesi distribution - North, South

Altitude $-0-1100 \mathrm{~m}$

Collection(s) - bb 19704; Forman 339; Koorders 17441, 17478, 17482, 17501; Teijsmann HB 13981

\section{Lindera spec.}

Sulawesi distribution - South

Lore Lindu National Park \& environs — present Altitude $-750 \mathrm{~m}$

Collection(s) - Van Balgooy 3560 
LITSEA

Litsea densiflora (Teschner) Kosterm.

Sulawesi distribution - Central, South

Lore Lindu National Park \& environs - present

Altitude - 800-1000 m

Collection(s) - Meijer 9672, 10027

Litsea diversifolia Blume

Sulawesi distribution - Central

Altitude $-200 \mathrm{~m}$

Collection(s) - Ramlanto \& Fanani 712

Litsea elliptica Blume

Vernacular name(s) - Ponto langio

Sulawesi distribution - North, South, SouthEast

Altitude - 20-750 m

Collection(s) - bb 21090, 28246; Cel III 61

Litsea ferruginia Blume

Vernacular name(s) - Bakkang boeloe; Laputankawok

Sulawesi distribution - South

Collection(s) - Teijsmann HB 14120

Litsea firma (Blume) Hook.f.

Vernacular name(s) - Alo'k

Sulawesi distribution - Central, South, SouthEast

Altitude - 5-1500 m

Collection(s) - bb 21382, 22619, 24171, 31829, 33068; Cel II 302; Ramlanto \& Fanani 714

\section{Litsea formanii Kosterm.}

Vernacular name(s) - Timbora dempad

Sulawesi distribution - North

Altitude - 340-900 m

Collection(s) - bb 13513, 17153; Forman 271; Koorders 17517, 17525

Litsea fulva (Blume) Vill.

Sulawesi distribution - North

Altitude $-500 \mathrm{~m}$

Collection(s) - Koorders 17431, 17457, 17493, 17505

\section{Litsea garciae Vidal}

Sulawesi distribution - South

Altitude $-430 \mathrm{~m}$
Collection(s) - bb 28278

Litsea glutinosa (Lour.) C.B. Rob.

Vernacular name(s) - Luno

Sulawesi distribution - North

Altitude - 0-5 m

Collection(s) - Koorders 17427; Lam 2452

Litsea grandis (Wall.) Hook.f.

Vernacular name(s) - Ponto oemera; Pontobaka-Pontopoe

Sulawesi distribution - North, South

Altitude - 200-1800 m

Collection(s) - bb 20864; Cel II 138, II 335, III 62; Koorders 17425

\section{Litsea hutchinsonii Merr.}

Sulawesi distribution - North

Altitude $-200 \mathrm{~m}$

Collection(s) - bb 19420

Litsea insignis (Blume) Boerl.

Vernacular name(s) - Pa midar

Sulawesi distribution - Central, South-East

Altitude - 125-645 m

Collection(s) - Elbert 3032; Noerkas 418

Litsea mappacea (Blume) Boerl.

Vernacular name(s) - ba' anah; Babaka / Baka poete; Bakan; Wakan [Tontembuan]; Wakkan

Sulawesi distribution - North, Central, South, South-East

Altitude - 0-800 m

Collection(s) - Alston 15834; bb 17066, 21364; Burley, Partomihardjo \& Rachman 3684; Cel V 240; Forman 346; Hose 196; Koorders $17421,17422,17423,17430,17438,17445$, 17454, 17471, 17473, 17485, 24066; Lam 2586; Meijer 9350; Noerkas 256, 495; Rachmat 578; Ramlanto \& Fanani 746

\section{Litsea noronhae Blume}

Sulawesi distribution - Central, South-East

Altitude - 100-350 m

Collection(s) - Prawiroatmodjo \& Maskuri 1377; Ramlanto \& Fanani 490, 511

Litsea ochracea (Blume) Boerl.

Sulawesi distribution - South

Collection(s) - Teijsmann HB 11780 


\section{Litsea oppositifolia Gibbs}

Vernacular name(s) - Nipil

Sulawesi distribution - North

Altitude $-400 \mathrm{~m}$

Collection(s) - De Vogel 2648

Litsea pallida (Blume) Boerl.

Vernacular name(s) - Kalokaloro; Timboon rintek

Sulawesi distribution - North, South

Altitude - $1200 \mathrm{~m}$

Collection(s) - bb 5161; Forman 364; Forsten 10,412

\section{Litsea robusta Blume}

Sulawesi distribution - Central

Altitude $-1000 \mathrm{~m}$

Collection(s) - Tantra 1531

\section{Litsea timoriana Span.}

Vernacular name(s) - abanbang'a; Monkoeni kaoe; Soejoe manaai; Wakan

Sulawesi distribution - North, Central, South

Altitude $-20-800 \mathrm{~m}$

Collection(s) - bb 14338, 28248; Cel V 183; De Vriese \& Teijsmann 98; Forman 227; Koorders 17428, 17434, 17436, 17461, 17484, 17496, 17497, 17499, 17500; Lam 3010; Teijsmann HB 12431, HB 12627, HB 13588

\section{Litsea tomentosa Blume}

Sulawesi distribution - North

Altitude - 500-1000 m

Collection(s) - Koorders 17466, 17467, 17472, 17495,17514

Litsea varians (Blume) Boerl.

Sulawesi distribution - North

Collection(s) - Alston 15927

\section{Litsea spec.}

Vernacular name(s) - Kali-bangkere; Ponto loijo

Sulawesi distribution - North, Central, South, South-East

Altitude - 20-2450 m

Collection(s) - bb 20748, 26269, 32454; Cel II 314, III 14, IV 144, IV 146, IV 147; De Joncheere 1133; De Vogel 2645, 2647, 5163 ,
5241, 5345, 5424, 5531, 5561, 5598, 5656; Forman 389; Johansson 413, 541; Keßler et al. PK 3153, PK 3166; Koorders 17409, $17411,17432,17435,17448,17449,17452$, 17458, 17503, 18137; Meijer 9426, 9622 , 10877, 10998, 11302; Prawiroatmodjo \& Soewoko 1702, 1866, 1893; Ramlanto \& Fanani 663, 679; Sands 327, 382; Tantra 1524, 1534, 1542, 1560, 1566; Teijsmann HB 12604; Van Balgooy 3033, 3099, 3160, 3263, $3286,3321,3376,3415,3416,3528,3792$, 3975; Yoshida 1113

\section{NEOLITSEA}

Neolitsea javanica (Blume) Backer

Sulawesi distribution - South

Collection(s) - Teijsmann HB 13959, HB 13989

Neolitsea latifolia (Blume) Moore

Vernacular name(s) - Panera

Sulawesi distribution - South

Collection(s) - Teijsmann HB 13671

Neolitsea villosa (Blume) Merr.

Sulawesi distribution - North

Altitude - $1200 \mathrm{~m}$

Collection(s) - Koorders 17462

\section{Neolitsea spec.}

Vernacular name(s) - Karaskas [Tombulu]

Sulawesi distribution - North, South, SouthEast

Altitude $-0-3000 \mathrm{~m}$

Collection(s) - Alston 16272; bb 22864; Chin 3633; De Vogel 2477, 6203; Elbert 3474, 3498; Eyma 3479; Yoshida 947

\section{NOTHAPHOEBE}

\section{Nothaphoebe albipes Kosterm.}

Vernacular name(s) - Ilarematoe moeta; Ilarematoe motaha

Sulawesi distribution - South

Altitude - 100-300 m

Collection(s) - Cel 323, II 111, II 121, II 368, II 422

\section{Nothaphoebe brevipaniculata Kosterm.}

Sulawesi distribution - South 
Altitude $-20 \mathrm{~m}$

Collection(s) - bb 23258

Nothaphoebe myrmecophila Kosterm.

Sulawesi distribution - North

Collection(s) - bb 129

Nothaphoebe patentinervis Kosterm.

Sulawesi distribution - South

Altitude $-1500 \mathrm{~m}$

Collection(s) - bb 19568

Nothaphoebe regalis Kosterm.

Sulawesi distribution - North

Altitude $-400 \mathrm{~m}$

Collection(s) - Koorders 17490

Nothaphoebe spec.

Vernacular name(s) - Anjoen poetih; Oöle motadoe

Sulawesi distribution - North, South, SouthEast

Altitude - 10-500 m

Collection(s) - bb 20759, 21320, 24193; Cel II 108, II 319, V 179; Koorders 17424, 17470; Lam 3564

\section{PERSEA}

Persea rimosa (Blume) Kosterm.

Sulawesi distribution - South

Altitude $-1100 \mathrm{~m}$

Collection(s) - Bünnemeyer 11812; Teijsmann HB 13672

Persea spec.

Sulawesi distribution - South

Altitude - $1425 \mathrm{~m}$

Collection(s) - Meijer 11023

PHOEBE

Phoebe cuneata Blume

Sulawesi distribution - North

Altitude $-500 \mathrm{~m}$

Collection(s) - De Vogel 2638

Phoebe forbesii Gamble

Sulawesi distribution - North
Altitude $-200-700 \mathrm{~m}$

Collection(s) - Koorders 17426, 17486, 17491, 24070

Phoebe grandis (Nees) Merr.

Vernacular name(s) - aripoengoe; Haripoengoe Sulawesi distribution - Central, North

Altitude - 200-340 m

Collection(s) - bb 13507; Ramlanto \& Fanani 724

Phoebe sterculioides (Elmer) Merr.

Sulawesi distribution - South, South-East

Altitude - 300-1500 m

Collection(s) — bb 21729, 24195, 28296

Phoebe tenuifolia Kosterm.

Vernacular name(s) - Aripoengoe/Alipoengo

Sulawesi distribution - North, South-East

Collection(s) - bb 2249

Phoebe spec.

Vernacular name(s) - Potingkea [Besoa/Napu]; Raripungu sela

Sulawesi distribution - North, Central, South

Altitude $-200-1000 \mathrm{~m}$

Collection(s) - bb 20894; De Vogel 5261; Koorders 17429, 17504; Meijer 9374; Van Balgooy 1579, 3079, 3136, 3526

\section{LECYTHIDACEAE}

\section{BARRINGTONIA}

Barringtonia acutangula (L.) Gaertn.

Vernacular name(s) - Ipil [Minahasa]; Poetja Sulawesi distribution - North, South, SouthEast

Altitude $-20-250 \mathrm{~m}$

Collection(s) - bb 18030; Bünnemeyer 10631; Cel I 59; Chin 3495, 3575, 3585, 3586; De Vogel 2444; Meijer 10834; Noerkas 371; Prawiroatmodjo \& Maskuri 1182; Prawiroatmodjo \& Soewoko 1797

Barringtonia acutangula (L.) Gaertn. subsp. spicata (Blume) Payens

Sulawesi distribution - North, Central, South Lore Lindu National Park \& environs - present Altitude $-20-50 \mathrm{~m}$ 
Collection(s) - De Vogel 2440, 2443, 2572, 2598, 5652; Soenarko 299

Barringtonia asiatica (L.) Kurz

Sulawesi distribution - North, South-East

Altitude - 0-196 m

Collection(s) - Elbert 2523; Prance 30096; Sawyer 196

Barringtonia niedenzuana (K. Schum.) Knuth

Vernacular name(s) - Buaro

Sulawesi distribution - North

Altitude $-100 \mathrm{~m}$

Collection(s) - Lam 2844

Barringtonia racemosa (L.) Spreng.

Vernacular name(s) - Parang'a; Poetah

Sulawesi distribution - North, South, SouthEast

Altitude $\longrightarrow$ 0-300 m

Collection(s) - bb 4185, 19694; Elbert 2858, 2917, 3417, 3441; Forsten 318; Koorders 18226; Lam 3226; Prawiroatmodjo \& Maskuri 1385, 1473; Prawiroatmodjo \& Soewoko 1915,1982

\section{Barringtonia spec.}

Sulawesi distribution - Central, South

Altitude - 70-600 m

Collection(s) - bb 24978; De Vogel 6009

\section{PLANCHONIA}

\section{Planchonia valida (DC.) Blume}

Vernacular name(s) - Ipil; Meoe; Saroe manggané; Wewoe

Sulawesi distribution - North, Central, South, South-East

Altitude - 10-900 m

Collection(s) - bb 3898, 4192, 13754, 17294 , 19424, 24994, 28737, 31823, 31867,32445 , 32478; Burley, Partomihardjo \& Rachman 3939; Cel I 11, V 232; Koorders 18223, 18225, 18227, 18232, 18274; Meijer 10734, 10944; Teijsmann HB 12664

\section{LEEACEAE}

\section{LEEA}

Leea aculeata Blume ex Spreng.

Vernacular name(s) - Mamali; Omomali [Kotamobagu]; Totombore [Buton]

Sulawesi distribution - North, South, SouthEast

Altitude $-0-750 \mathrm{~m}$

Collection(s) - Alston 16002; bb 17564; De Vogel 2467; De Vogel \& Vermeulen 6806, 6839, 6917; Elbert 2806, 2829, 2870, 2936; Forman 293; Koorders 15877, 15878, 15879, $15880,15881,15882$; Lam 2415, 2729; Whitmore \& Sidiyasa TCW 3375, TCW 3428; Widjaja 547; Yusuf \& Wahyono 19

\section{Leea aequata $L$.}

Vernacular name(s) - Silampawakoe

Sulawesi distribution - North, Central, South, South-East

Altitude - 10-650 m

Collection(s) - Amir 188; Buwalda 3797; Coode 6243; De Vogel 5897; De Vogel \& Vermeulen 6755; Johansson 193; Koorders 15884; Meijer 10894; Prawiroatmodjo \& Maskuri 1086, 1478

Leea angulata Korth. ex Miq.

Vernacular name(s) - Kaae Ohio

Sulawesi distribution - North, South

Altitude - $40 \mathrm{~m}$

Collection(s) - Cel V 159; De Vogel 2599; Meijer 10733

\section{Leea guineensis $\mathrm{G}$. Don}

Vernacular name(s) - Mamali wawine

Sulawesi distribution - North, Central, SouthEast

Altitude - 0-1250 m

Collection(s) - De Vogel \& Vermeulen 6776; Elbert 2756, 3383, 3438; Hennipman 5610; Johansson 203; Koorders 15895; Lam 2472, 3074

Leea indica (Burm.f.) Merr.

Vernacular name(s) - Mamali [Talaud]; Taccalea [Kendari]

Sulawesi distribution - North, Central, South Lore Lindu National Park \& environs - present 
Altitude $-75-1200 \mathrm{~m}$

Collection(s) - Bloembergen 4254; De Vogel 5235; Forsten 103; Hennipman 5938; Koorders 15893, 15894, 24075; Lam 2539; Meijer 9704

\section{Leea philippinensis Merr.}

Sulawesi distribution - North

Altitude $-200 \mathrm{~m}$

Collection(s) - De Vogel 2471

\section{Leea rubra Blume}

Vernacular name(s) - Mali mali

Sulawesi distribution - Central, South

Altitude - 50-1000 m

Collection(s) - Bünnemeyer 10578; Chin 3431; De Vogel 5536; Johansson 16; Noerkas 27; Rachmat 18

\section{Leea smithii Koord.}

Sulawesi distribution - North

Altitude - 650-700 m

Collection(s) - Koorders 15876, 15888

\section{Leea spec.}

Sulawesi distribution - Central, South Altitude - 80-500 m

Collection(s) - Coode 6073; De Vogel 5675; Ramlanto 111

\section{LEGUMINOSAE}

\section{LEGUMINOSAE-CAESALPINIOIDEAE}

\section{BAUHINIA}

Bauhinia lingua DC. var. riedelii (Baker) K. Larsen \& S. S. Larsen

Sulawesi distribution - North

Altitude $-50 \mathrm{~m}$

Collection(s) - Koorders 17564

\section{Bauhinia spec.}

Sulawesi distribution - Central, South, SouthEast

Lore Lindu National Park \& environs - present Altitude - 50-1300 m

Collection(s) - Kofman 137; Prawiroatmodjo \& Maskuri 1230; Van Balgooy 3548

\section{CAESALPINIA}

Caesalpinia bonduc (L.) Roxb.

Vernacular name(s) - Ajamboe nonoh koratj Sulawesi distribution - North

Collection(s) — Koorders 17627, 17628; Rachmat 353

\section{Caesalpinia crista $\mathrm{L}$.}

Vernacular name(s) - Djamboe djini

Sulawesi distribution - North, Central, South Lore Lindu National Park \& environs - present Altitude $-0-600 \mathrm{~m}$

Collection(s) - Elbert 2628, 2935; Koorders 17707, 17709; Meijer 9287; Rachmat 808; Van Steenis 10330

Caesalpinia latisiliqua (Cav.) Hattink

Vernacular name(s) - Konga mowehi [Kendari] Sulawesi distribution - North, South

Altitude - 400-600 m

Collection(s) - De Vogel 5750; Kaudern 254; Van Balgooy 3918

Caesalpinia major (Medik.) Dandy \& Exell

Sulawesi distribution - Central, South

Altitude - 400-1000 m

Collection(s) - De Vogel 5185, 5839

Caesalpinia pulcherrima Sw.

Sulawesi distribution - Central, South

Altitude $-60 \mathrm{~m}$

Collection(s) - Amir 233

\section{Caesalpinia spec.}

Vernacular name(s) - pehi

Sulawesi distribution - Central, South-East

Altitude $-50-300 \mathrm{~m}$

Collection(s) - Meijer 10158; Widjaja 661

\section{CASSIA}

\section{Cassia alata L.}

Sulawesi distribution - North, South, SouthEast

Altitude - 0-950 m

Collection(s) - Bünnemeyer 10704, 10974, 11354; De Vogel 6279; Elbert 3228; Eyma 1093; Koorders 17575; Prawiroatmodjo \& Maskuri 1174 


\section{Cassia fistula $L$.}

Vernacular name(s) - Kapoeé poeé

Sulawesi distribution - South, South-East

Altitude - 0-200 m

Collection(s) - bb 5859; Elbert 2611, 2613; Van der Vlies 29

\section{Cassia floribunda Cav.}

Sulawesi distribution - North

Altitude - 800-900 m

Collection(s) - Forman 196

\section{Cassia fruticosa Mill.}

Vernacular name(s) - Tangke malu

Sulawesi distribution - North, South, SouthEast

Altitude - 30-150 m

Collection(s) - Eyma 3411; Prawiroatmodjo \& Maskuri 1203; Widjaja 682

\section{Cassia mimosoides L.}

Sulawesi distribution - North, South

Altitude $-15-100 \mathrm{~m}$

Collection(s) - Forsten 63; Lam 3024, 3423; Monod de Froideville 282; Noerkas 254, 283

Cassia occidentalis L.

Vernacular name(s) - tanduru manu

Sulawesi distribution - North, Central, South, South-East

Altitude - 0-75 m

Collection(s) - Elbert 2620; Kartawinata 1292; Kaudern 453; Koorders 17576, 17577, 17578; Noerkas 15; Widjaja 718; Yoshida 871

Cassia pumila Lam.

Sulawesi distribution - South, South-East

Altitude $-0-100 \mathrm{~m}$

Collection(s) - Bünnemeyer 10689; Elbert 6358

Cassia siamea Lam.

Sulawesi distribution - Central

Lore Lindu National Park \& environs - present

Altitude $-0-30 \mathrm{~m}$

Collection(s) - Hennipman 5022; Kaudern 513

Cassia timorensis DC.

Vernacular name(s) - Melanpessi; Morohopa Sulawesi distribution - South, South-East
Altitude - 750-950 m

Collection(s) - Bünnemeyer 11110, 11441; Meijer 10712, 10714; Noerkas 195, 242; Widjaja 678

\section{Cassia tora L.}

Sulawesi distribution - North, South, SouthEast

Altitude - 0-950 m

Collection(s) - Bünnemeyer 11106, 11379; Elbert 6284; Koorders 17579

\section{Cassia spec.}

Sulawesi distribution - North, Central

Altitude - $1060 \mathrm{~m}$

Collection(s) - De Joncheere 1021; KeBler et al. PK 3139

\section{CATHORMION}

Cathormion umbellatum (Vahl) Kosterm.

Sulawesi distribution - South

Altitude $-1 \mathrm{~m}$

Collection(s) - bb 19696; Noerkas 52, 67; Yusuf \& Wahyono 36

Cathormion umbellatum (Vahl) Kosterm. subsp. moniliforme (DC.) Brummitt

Sulawesi distribution - South

Collection(s) - Teijsmann HB 13720; Yoshida 903

\section{CYNOMETRA}

\section{Cynometra cauliflora L.}

Vernacular name(s) - Namu (Buton)

Sulawesi distribution - North, South, SouthEast

Altitude - 260-300 m

Collection(s) - Cel V 233; Coode 6280; Meijer 10838; Whitmore \& Sidiyasa TCW 3406

\section{Cynometra ramiflora $\mathrm{L}$.}

Vernacular name(s) - Katji-katji

Sulawesi distribution - North, South, SouthEast

Altitude - 0-257 m

Collection(s) - Afriastini 2048; bb 21762 , 22953; Cel I 52; Elbert 3336; Kjellberg 1262, 2422; Koorders 17588 


\section{Cynometra spec.}

Sulawesi distribution - South

Collection(s) - Meijer 10894

\section{GLEDITISIA}

\section{Gleditsia rolfei S. Vidal}

Sulawesi distribution - North

Altitude - 0-500 m

Collection(s) - Koorders 16855, 16856, 16858, $16859,16860,16861,29480$

\section{INTSIA}

\section{Intsia bijuga (Colebr.) Kuntze}

Vernacular name(s) - Ipi; Loroenoe

Sulawesi distribution - North, Central, South, South-East

Altitude $-0-70 \mathrm{~m}$

Collection(s) - bb 3918, 15081, 15370, 28734, 31493, 31524, 31861, 31902; Cel III 47, III 48, III 97; Ham B 5, L 3; Koorders 17554, 17555, 17556, 17559, 17560; Meijer 9252; Prance 30098

Intsia palembanica Miq.

Sulawesi distribution - South, South-East

Altitude - 10-60 m

Collection(s) - bb 19692, 21095, 21111, 23301, 32460; Heyne 2855; Noerkas 370

\section{KALAPPIA}

Kalappia celebica Kosterm.

Sulawesi distribution - South

Altitude - $10 \mathrm{~m}$

Collection(s) - bb 33693

\section{MANILTOA}

Maniltoa schefferi K. Schum.

Vernacular name(s) - Lamoeta

Sulawesi distribution - North, Central, South, South-East

Altitude - 6-200 m

Collection(s) - bb 15125, 17201, 19591, 19701, $31858,31887,31918,32330$

\section{PELTOPHORUM}

Peltophorum pterocarpum (DC.) Backer ex K. Heyne

Vernacular name(s) - Molilipota; Soga

Sulawesi distribution - North, South, SouthEast

Altitude $-0-75 \mathrm{~m}$

Collection(s) - bb 3921, 13744, 15130, 17195, 24069, 31921, 32574; Elbert 2659; Koorders 17651, 17652, 17653; Teijsmann HB 11844, HB 13848

\section{SARACA}

Saraca celebica W.J. de Wilde

Sulawesi distribution - North, Central, South Altitude $-0-200 \mathrm{~m}$

Collection(s) - Coode 5801; Eyma 3943; Van Balgooy 3785

\section{SENNA}

Senna obtusifolia (L.) Irwin \& Barneby

Sulawesi distribution - North

Altitude - $220 \mathrm{~m}$

Collection(s) - De Vogel \& Vermeulen 6481

\section{SINDORA}

Sindora galedupa Prain

Vernacular name(s) - Mombingo

Sulawesi distribution - North, South-East

Altitude $-0-60 \mathrm{~m}$

Collection(s) - bb 15375; Elbert 2543

\section{LEGUMINOSAE-MIMOSOIDEAE}

ACACIA

Acacia farnesiana (L.) Willd.

Sulawesi distribution - Central

Collection(s) - Kaudern 486; Noerkas 413

\section{ADENANTHERA}

Adenanthera pavonina $\mathrm{L}$.

Vernacular name(s) - Wela poeté, Konsi we Sulawesi distribution - Central, South 
Altitude - 25-400 m

Collection(s) - bb 15375; Cel I 29, II 360, IV

118; De Vogel 5898; Kjellberg 961; Meijer 10856; Van Balgooy 3889

\section{ALBIZIA}

Albizia procera (Roxb.) Benth.

Vernacular name(s) - Kajoe bilabang; konturi Sulawesi distribution - South, South-East Altitude - 37-280 m

Collection(s) - bb 20254, 24708; Bünnemeyer 10681, 10768; Noerkas 282; Widjaja 688; Wieringa 1829

\section{Albizia rufa Benth.}

Sulawesi distribution - South

Altitude $-0-5 \mathrm{~m}$

Collection(s) — Hidayat AH 304

Albizia saponaria (Lour.) Blume ex Miq.

Vernacular name(s) - Hangi; Langeri; Langiri boesa; Langík'a; Lendaki; Wilaloto-kaoe

Sulawesi distribution - North, Central, South, South-East

Altitude - 100-900 m

Collection(s) - bb 5601, 15082, 16988, 22063, 24705, 25008; Bünnemeyer 10632, 10893, 11299; Cel I 72, V 137, V 138, V 322, V 330; Coode 5874; Koorders 17546, 17547, 17549, 17550; Lam 2565, 3028, 3045; McDonald \& Ismail 3869; Meijer 10225, 10676; Milliken 851; Teijsmann HB 12300

\section{ARCHIDENDRON}

Archidendron clypearia (Jack) I.C. Nielsen

Sulawesi distribution - North

Collection(s) - Koorders 17661

Archidendron clypearia (Jack) I.C. Nielsen var. velutinum (Merr. \& Perry) I.C. Nielsen

Sulawesi distribution - South

Altitude - $400 \mathrm{~m}$

Collection(s) - De Vogel 6190

Archidendron crateradenum (Kosterm.)

I.C. Nielsen

Sulawesi distribution - North

Collection(s) - Koorders 17703
Archidendron minahassae (Teijsm. \& Binn. ex Koord.) I.C. Nielsen

Sulawesi distribution - North

Altitude $-50-500 \mathrm{~m}$

Collection(s) - Koorders 17662, 17663, 17664, 17703,19720

\section{Archidendron pauciflorum (Benth.)}

I.C. Nielsen

Sulawesi distribution - North, South, SouthEast

Altitude - 0-1200 m

Collection(s) - bb 5850; Elbert 3288, 3299; Koorders 17665; Meijer 9702, 10947; Noerkas 220

\section{Archidendron spec.}

Sulawesi distribution - North, Central

Altitude $-220-600 \mathrm{~m}$

Collection(s) - De Vogel \& Vermeulen 6469; Wieringa 1849

\section{LEUCAENA}

Leucaena leucocephala (Lam.) de Wit

Sulawesi distribution - North, Central, South Altitude - 0-1040 m

Collection(s) - Bünnemeyer 11298; Eyma 1741; Hidayat AH 288; Kaudern 427; Weir 17

\section{MIMOSA}

Mimosa pudica L. var. hispida Brenan

Sulawesi distribution - South

Altitude $-50 \mathrm{~m}$

Collection(s) - Buwalda 3736

\section{PARKIA}

Parkia sumatrana Miq. subsp. sumatrana

Sulawesi distribution - South-East

Altitude $-25 \mathrm{~m}$

Collection(s) - bb 21099, 21727

Parkia timoriana (DC.) Merr.

Sulawesi distribution - Central, South-East

Altitude - 25-300 m

Collection(s) - bb 25001; Coode 6266 


\section{PITHECELLOBIUM}

\section{Pithecellobium spec.}

Sulawesi distribution - South-East

Altitude $-20-250 \mathrm{~m}$

Collection(s) - Amir 235; Prawiroatmodjo \& Soewoko 1615, 1659, 1683, 1728

\section{SERIANTHES}

\section{Serianthes dilmyi Fosb.}

Vernacular name(s) - Molilipota lodeheto Sulawesi distribution - North, Central

Lore Lindu National Park \& environs - present Altitude - 0-15 m

Collection(s) - bb 15604, 28735

Serianthes minahassae (Koord.) Merr. \& Perry

Vernacular name(s) - Tedoehoe poeli

Sulawesi distribution - North, South

Altitude - 30-500 m

Collection(s) - bb 20807, 23912, 32614, 32805, 32807, 32811; Cel V 225, V 370; Koorders $17541,17542,17543,17552,17650,17654$, $22617,22620,29482$

Serianthes minahassae (Koord.) Merr. \& Perry subsp. minahassae

Sulawesi distribution - South

Altitude - 40-50 m

Collection(s) - bb 32806, 32808, 32809, 32810

\section{WALLACEODENDRON}

Wallaceodendron celebicum Koord.

Vernacular name(s) - Latoela

Sulawesi distribution - North

Altitude $-50 \mathrm{~m}$

Collection(s) — bb 15378; Heyne 2848; Koorders $17537,17538,17553,29481$

\section{LEGUMINOSAE-PAPILIONOIDEAE}

\section{CAJANUS}

Cajanus cajan Millsp.

Vernacular name(s) - Binta Acening

Sulawesi distribution - South

Altitude - 400-1200 m
Collection(s) - Bünnemeyer 11768, 12584; De Vogel 6104

\section{CALOPOGONIUM}

Calopogonium mucunoides Desv.

Sulawesi distribution - North, Central, South, South-East

Altitude - 80-1000 m

Collection(s) - De Vogel 6487; Johansson 580; Meijer 9352; Monod de Froideville 52; Van Balgooy 3576; Widjaja 618

\section{CANAVALIA}

\section{Canavalia cathartica Thouars}

Sulawesi distribution - North, Central, South

Altitude $-0-400 \mathrm{~m}$

Collection(s) - Chin 3537; De Vogel 5911; Eyma 3532; Koorders 17570, 17674; Rachmat 302; Ramlanto 162; Van Balgooy 3757

Canavalia maritima (Aubl.) Thouars

Vernacular name(s) - Pico-pico

Sulawesi distribution - North, South, SouthEast

Altitude -0-60 m

Collection(s) - bb 3631; Elbert 2533; Hidayat AH 274; Kartawinata 1271; Koorders 17567 , 17571; Monod de Froideville 408; SnelliusII $11300 \mathrm{~A}$

\section{CLITORIA}

\section{Clitoria ternatea L.}

Sulawesi distribution - North, Central, South, South-East

Altitude - 50-600 m

Collection(s) - Forsten 433, 434; Kaudern 116, 371; Kjellberg 143; Koorders 17571; Meijer 10189; Monod de Froideville 51; Prawiroatmodjo \& Maskuri 1263

\section{CROTALARIA}

Crotalaria acicularis Ham. ex Benth.

Sulawesi distribution - South

Altitude $-800 \mathrm{~m}$

Collection(s) - Wieringa 1873 


\section{Crotalaria albida L.}

Sulawesi distribution - South

Altitude - $1400 \mathrm{~m}$

Collection(s) - Bünnemeyer 12610; Zollinger 2482

\section{Crotalaria calycina Schrank}

Sulawesi distribution - North, South, SouthEast

Altitude $-50-580 \mathrm{~m}$

Collection(s) - Kjellberg 92; Koorders 17586; Monod de Froideville 6, 480; Noerkas 25

\section{Crotalaria ferruginea Graham}

Sulawesi distribution - North, South

Altitude - 350-1330 m

Collection(s) - Bünnemeyer 11210; Eyma 3456; Kaudern 321; Monod de Froideville $2,4,414,481$

\section{Crotalaria linifolia L.f.}

Vernacular name(s) - nid djobong

Sulawesi distribution - North, Central, South Lore Lindu National Park \& environs - present Altitude - 0-1300 m

Collection(s) - Bünnemeyer 10946, 11069; Eyma 3421; Kjellberg 101; Meijer 9193; Monod de Froideville 79, 86, 104; Noerkas 45; Rachmat 112; Van Steenis 10398

\section{Crotalaria pallida Aiton}

Vernacular name(s) - roekoe-roekoe

Sulawesi distribution - North, Central, South Altitude - 50-950 m

Collection(s) - Bünnemeyer 10602, 10955; Kaudern 16, 395; Koorders 17583; Monod de Froideville 416; Rachmat 28, 256

Crotalaria prostrata Rottler ex Willd.

Sulawesi distribution - South-East

Altitude $-30 \mathrm{~m}$

Collection(s) - Monod de Froideville 74

\section{Crotalaria quinquefolia $\mathrm{L}$.}

Sulawesi distribution - South

Collection(s) - Monod de Froideville 1; Rachmat 104

\section{Crotalaria retusa $\mathrm{L}$.}

Sulawesi distribution - North, South
Altitude $-1000 \mathrm{~m}$

Collection(s) - Bloembergen 4089; Kaudern 113; Koorders 17585; Monod de Froideville 415; Noerkas 42; Rachmat 147; Robinson 2464

\section{Crotalaria sessiflora L.}

Sulawesi distribution - South

Altitude - 400-800 m

Collection(s) - Monod de Froideville 5, 482

\section{Crotalaria verrucosa $L$.}

Sulawesi distribution - South, South-East

Altitude - 0-950 m

Collection(s) - Bünnemeyer 11341; Elbert 2642; Eyma 3333

\section{Crotalaria zanzibarica Benth.}

Sulawesi distribution - North, South, SouthEast

Altitude - 150-450 m

Collection(s) - Alston 16417; Eyma 4182; Kofman 191; Prawiroatmodjo \& Maskuri 1268

\section{Crotalaria spec.}

Sulawesi distribution - North, Central

Altitude - 220-650 m

Collection(s) - De Vogel 5640; De Vogel \& Vermeulen 6930; Johansson 361

\section{DALBERGIA}

Dalbergia candenatensis (Dennst.) Prain

Sulawesi distribution - North

Altitude $-5 \mathrm{~m}$

Collection(s) - Lam 2493

Dalbergia discolor Blume ex Miq.

Sulawesi distribution - North, South-East

Altitude - 0-250 m

Collection(s) - Elbert 3059, 3074; Milliken 852; Prawiroatmodjo \& Soewoko 1783; Rachmat 718

\section{Dalbergia ferruginea Roxb.}

Vernacular name(s) - Aja-Aja pakampi; sarumpaniran

Sulawesi distribution - North, South-East

Altitude $-0-300 \mathrm{~m}$ 
Collection(s) - Elbert 2831, 3002, 3068, 3294;

Forman 408; Kaudern 528; Koorders 17589;

Lam 2601, 3048; Noerkas 179

Dalbergia pinnata (Lour.) Prain

Sulawesi distribution - Central

Lore Lindu National Park \& environs - present

Altitude $-800 \mathrm{~m}$

Collection(s) - Meijer 10205

Dalbergia spec.

Sulawesi distribution - South

Collection(s) - Chin 3498

\section{DENDROLOBIUM}

Dendrolobium cumingianum Benth.

Sulawesi distribution - South

Altitude $-0-5 \mathrm{~m}$

Collection(s) - Hidayat AH 259

\section{DERRIS}

Derris cebuensis Merr.

Sulawesi distribution - South-East

Altitude $-250-500 \mathrm{~m}$

Collection(s) - McDonald \& Ismail 4181

\section{DESMODIUM}

Desmodium securiforme Benth.

Sulawesi distribution - North

Collection(s) - Lam 2637

Desmodium sequax Wall.

Sulawesi distribution - South

Altitude $-2000 \mathrm{~m}$

Collection(s) - Wieringa 1890

Desmodium spec.

Sulawesi distribution - North

Altitude - 350-750 m

Collection(s) - Burley, Partomihardjo \& Rachman 3750

\section{DIOCLEA}

Dioclea hexandra (Ralph) Mabb.

Sulawesi distribution - North
Altitude $-150 \mathrm{~m}$

Collection(s) - Burley, Partomihardjo \& Rach$\operatorname{man} 4270$

\section{ERYTHRINA}

Erythrina subumbrans (Hassk.) Merr.

Sulawesi distribution - North, South, SouthEast

Altitude - 10-1000 m

Collection(s) - bb 21354; Koorders 17625; Monod de Froideville 293

\section{Erythrina variegata $L$.}

Vernacular name(s) - Kane; Ra-ra

Sulawesi distribution - North, South, SouthEast

Altitude - 0-900 m

Collection(s) - bb 21789; Bünnemeyer 12578; Elbert 2666; Kaudern 401; Koorders 17624; Rachmat 254

\section{FLEMINGIA}

Flemingia involucrata Benth.

Sulawesi distribution - South

Altitude $-70 \mathrm{~m}$

Collection(s) - Bünnemeyer 10680

Flemingia lineata Roxb.

Sulawesi distribution - South

Collection(s) - Monod de Froideville 97

Flemingia macrophylla (Willd.) Kuntze

Sulawesi distribution - South, South-East

Altitude - 20-400 m

Collection(s) - Elbert 3448; Monod de Froideville $62,299,495$

Flemingia strobilifera (L.) R.Br.

Vernacular name(s) - apa-apa (Moronene); Kasappe

Sulawesi distribution - North, Central, South, South-East

Altitude - 0-1000 m

Collection(s) - Alston 15767; Bünnemeyer 12576; De Vogel 5884; Elbert 2636, 2786 , 2880, 3400; Koorders 17626; Monod de Froideville 61; Rachmat 303; Widjaja 653; Yoshida 1336 


\section{Flemingia spec.}

Sulawesi distribution - North

Altitude - $220 \mathrm{~m}$

Collection(s) - De Vogel \& Vermeulen 6921

\section{GLIRICIDIA}

Gliricidia sepium (Jacq.) Steud.

Sulawesi distribution - North

Altitude $-1000 \mathrm{~m}$

Collection(s) - De Vogel \& Vermeulen 7149

\section{INDIGOFERA}

Indigofera galegoides DC.

Sulawesi distribution - South

Altitude $\longrightarrow 50 \mathrm{~m}$

Collection(s) - Bünnemeyer 10567

\section{Indigofera hirsuta $\mathrm{L}$.}

Vernacular name(s) - Febanang andjah

Sulawesi distribution - Central, South

Lore Lindu National Park \& environs - present

Altitude - 2-950 m

Collection(s) - Bünnemeyer 11224; Meijer 10196; Monod de Froideville 12; Noerkas 89; Rachmat 110

\section{Indigofera linnaei Ali}

Sulawesi distribution - Central

Lore Lindu National Park \& environs - present

Collection(s) - Meijer 9221

Indigofera spicata Forssk.

Sulawesi distribution - South

Altitude $-70-300 \mathrm{~m}$

Collection(s) - Bünnemeyer 10669; Monod de Froideville 13; Teijsmann HB 12373

\section{Indigofera tinctoria $\mathrm{L}$.}

Sulawesi distribution - South, South-East

Altitude - 0-75 m

Collection(s) - Elbert 2669; Monod de Froideville 9

\section{Indigofera trifoliata $\mathrm{L}$.}

Sulawesi distribution - South, South-East Altitude - 0-400 m

Collection(s) - Elbert 2597; Monod de Froideville 10,11
Indigofera zollingeriana Miq.

Sulawesi distribution — North

Altitude $-700 \mathrm{~m}$

Collection(s) - Koorders 17631; Monod de Froideville 96

\section{Indigofera spec.}

Sulawesi distribution - Central, North

Collection(s) - Alston 16425

\section{INOCARPUS}

Inocarpus fagifer (Parkinson) Fosberg

Vernacular name(s) - Iama; Katinda Angkaing; Kiama

Sulawesi distribution - North, South, SouthEast

Altitude - 5-300 m

Collection(s) - bb 4991; Elbert 2769; Lam 2876, 3359; Teijsmann HB 13623, HB 13850 , HB 13874

\section{MACROPSYCHANTHUS}

Macropsychanthus dolichobotrys Holthuis

Sulawesi distribution - North

Altitude $-20 \mathrm{~m}$

Collection(s) - Lam 3002

\section{MASTERSIA}

Mastersia bakeri Backer ex Koord.

Sulawesi distribution - North

Altitude - 0-200 m

Collection(s) - Koorders 17702, 17705

\section{MILLETTIA}

Millettia pinnata (L.) Panigrahi

Vernacular name(s) - Belalong balo; Kamoenjawa; La-ittah

Sulawesi distribution - North, Central, South, South-East

Altitude - 0-600 m

Collection(s) - bb 3686, 5862, 16985, 24068; Coode 5811; Elbert 3279; Kjellberg 519, 775; Koorders 17669, 17670, 17672, 17673; Lam 2499; Monod de Froideville 100, 302; Teijsmann HB 13894, HB 13909 
MUCUNA

\section{Mucuna spec.}

Sulawesi distribution - South-East

Altitude - 700-900 m

Collection(s) - McDonald \& Ismail 4003

ORMOSIA

\section{Ormosia calavensis Azaola}

Vernacular name(s) - Nakimbawai

Sulawesi distribution - North, Central, South

Altitude - 2-1200 m

Collection(s) - bb 19558, 19645, 19819, 29481; Lam 3073, 3296

Ormosia macrodisca Baker

Sulawesi distribution - North

Altitude - $450 \mathrm{~m}$

Collection(s) - Koorders 17685

\section{PTEROCARPUS}

Pterocarpus indicus Willd.

Vernacular name(s) - Adju ajenxana; cendana [Buton], asana; linggoeae; kaju Oelé; Tjempaga

Sulawesi distribution - North, Central, South, South-East

Altitude - 2-1100 m

Collection(s) - bb 5569, 19599, 21739, 23536, $23537,24126,24188,24659,25007,31522$, 32485, 32522, 32610; Cel I 13, III 37; De Vogel 5873; Ham R 7; Heyne 2822; Koorders 17680, 17681, 17682, 17683, 17684; Noerkas 169; Teijsmann HB 12671; Widjaja 660; Zeef 301

\section{PUERARIA}

Pueraria pulcherrima (Koord.) Merr.

Sulawesi distribution - North

Collection(s) - Koorders 17699

\section{SOPHORA}

\section{Sophora tomentosa L.}

Sulawesi distribution - South

Altitude $-0-5 \mathrm{~m}$

Collection(s) - Hidayat AH 269

\section{SPATHOLOBUS}

\section{Spatholobus spec.}

Sulawesi distribution - Central Lore Lindu National Park \& environs - present Altitude - 1040-1060 m

Collection(s) - Keßler et al. PK 3146, PK 3160

\section{STRONGYLODON}

\section{Strongylodon celebicus S.F. Huang}

Sulawesi distribution - Central

Lore Lindu National Park \& environs - present

Altitude $-1000 \mathrm{~m}$

Collection(s) - Van Balgooy 3422

TEPHROSIA

Tephrosia zollingeri Backer

Sulawesi distribution - South

Collection(s) - Zollinger 3321

VIGNA

Vigna marina (Burm.f.) Merr.

Sulawesi distribution - South

Altitude $-0-5 \mathrm{~m}$

Collection(s) - Hidayat AH 271, AH 302

\section{ZORNIA}

Zornia diphylla (L.) Pers.

Sulawesi distribution - South

Altitude $-10 \mathrm{~m}$

Collection(s) - Monod de Froideville 42; Noerkas 54; Zollinger 3305

\section{LILIACEAE}

\section{DRACAENA}

\section{Dracaena spec.}

Sulawesi distribution - Central

Lore Lindu National Park \& environs - present Altitude $-1100 \mathrm{~m}$

Collection(s) - KeBler et al. PK 3155 


\section{LOGANIACEAE}

\section{FAGRAEA}

\section{Fagraea auriculata Jack}

Sulawesi distribution - South

Altitude $-400 \mathrm{~m}$

Collection(s) - De Vogel 5722

Fagraea blumei G. Don

Vernacular name(s) - Nunuo [Suwawa]

Sulawesi distribution - North, Central

Altitude - 405-2200 m

Collection(s) - bb 13514; De Vogel 2479; Milliken 886; Van Balgooy 3282

Fagraea ceilanica Thunb.

Sulawesi distribution - North, Central, South Altitude - 0-1000 m

Collection(s) - Alston 16349; De Vogel 5574, 6385; Eyma 4127; Hennipman 5809; Ramlanto 176

Fagraea fragrans Roxb.

Vernacular name(s) - Bitjoro; Kolahi; Kulahi Sulawesi distribution - North, Central, South, South-East

Altitude - 25-500 m

Collection(s) - bb 4990, 13586, 20210, 26301, 26302, 26303, 31525, 32356; Cel IV 86, IV 136; Eyma 4060; Ham L 6; Heyne 2863; Noerkas 222; Van Balgooy 3988

Fagraea graciliflora Leenh.

Sulawesi distribution - Central

Lore Lindu National Park \& environs - present Altitude - 2050-2100 m

Collection(s) - De Vogel 5390; Van Balgooy 3247

\section{Fagraea longiflora Merr.}

Sulawesi distribution - North

Altitude - 900-1200 m

Collection(s) - Koorders 17734, 17735

Fagraea racemosa Wall.

Sulawesi distribution - South

Altitude - 320-970 m

Collection(s) - Meijer 9673; Noerkas 446; Ramlanto 189; Yusuf \& Wahyono 160
Fagraea tacapala Leenh. subsp. ceramensis Leenh.

Sulawesi distribution - South

Altitude - 1000-1150 m

Collection(s) - Hennipman 6061

Fagraea tacapala Leenh. subsp. gracilis Leenh.

Sulawesi distribution - North, Central, South Lore Lindu National Park \& environs - present Altitude - 970-1200 m

Collection(s) - Bloembergen 4068; De Vogel 5513; Kjellberg 1521a; Meijer 9711, 9945

Fagraea tacapala Leenh. subsp. tacapala

Sulawesi distribution - South

Altitude - 1600-2000 m

Collection(s) - Bünnemeyer 12100; Teijsmann HB 13665

\section{Fagraea spec.}

Sulawesi distribution - South

Altitude - $1700 \mathrm{~m}$

Collection(s) - Cel 226

\section{GENIOSTOMA}

Geniostoma rupestre J.R. Forst. \& G. Forst.

Vernacular name(s) - Abolo'a

Sulawesi distribution - North, South, SouthEast

Altitude $-50-3300 \mathrm{~m}$

Collection(s) - bb 14126; Bogor Botanical Garden 5777; Cel 15, V 272; Chin 3641; Elbert 2713, 3432; Eyma 470, 584, 679; Koorders 18713, 18721, 18722, 24063; Lam 2571, 3133, 3268; McDonald \& Ismail Cat 4031; Teijsmann HB 14000

\section{NEUBURGIA}

Neuburgia celebica (Koord.) Leenh.

Vernacular name(s) - A'asili; Danoan; Lea-lea; Lokabak [Kotamobagu]; Rèwè, Tamaba, keoe

Sulawesi distribution - North, Central, South, South-East

Altitude - 2-800 m

Collection(s) - bb 2242, 5434, 14219; Burley, Partomihardjo \& Rachman 3682, 3872; Cel III 40; De Vogel \& Vermeulen 6988; 
Koorders 18741; Lam 2973; Meijer 9403, 10784, 10909

\section{STRYCHNOS}

\section{Strychnos axillaris Colebr.}

Vernacular name(s) - Apoetóe laäwan

Sulawesi distribution - North, Central, South, South-East

Altitude - 100-1400 m

Collection(s) - De Vogel 5117, 5844, 6026, 6295; Hennipman 5811, 5820; Lam 3333; Prawiroatmodjo \& Soewoko 1907; Teijsmann HB 11754, HB 12131, HB 13607

Strychnos minor Benth.

Sulawesi distribution - North, South, SouthEast

Altitude $-0-750 \mathrm{~m}$

Collection(s) - Burley, Partomihardjo \& Rachman 3559, 3880; Church \& Ismail 12; De Vogel 2583; Elbert 3316; Koorders 17740; Rachmat 652; Teijsmann HB 12518

\section{LYTHRACEAE}

\section{AMMANNIA}

\section{Ammannia baccifera L.}

Sulawesi distribution - South

Collection(s) - Robinson 2462

Ammannia microcarpa DC.

Sulawesi distribution - South

Altitude $-950-1000 \mathrm{~m}$

Collection(s) - Bünnemeyer 11104, 11402; Noerkas 317

\section{LAGERSTROEMIA}

Lagerstroemia ovalifolia Teijsm. \& Binn.

Sulawesi distribution - North

Altitude - $40 \mathrm{~m}$

Collection(s) - De Vogel 2573

\section{Lagerstroemia piriformis Koehne}

Sulawesi distribution - North

Altitude $-10-700 \mathrm{~m}$

Collection(s) - Forsten 165; Koorders 17758, $17760,17761,17762,17763,17764$

\section{Lagerstroemia speciosa (L.) Pers.}

Vernacular name(s) - Ajopeng; Lobani

Sulawesi distribution - North, Central, South, South-East

Altitude - 15-60 m

Collection(s) - Amir 45; bb 20017, 21605; Noerkas 134, 275; Teijsmann HB 11815

\section{Lagerstroemia spec.}

Sulawesi distribution - South-East

Altitude - 20-250 m

Collection(s) - Widjaja 664

\section{LAWSONIA}

Lawsonia inermis $\mathrm{L}$.

Sulawesi distribution - Central

Lore Lindu National Park \& environs - present

Altitude $-30 \mathrm{~m}$

Collection(s) - Van Balgooy 2981

\section{PEMPHIS}

Pemphis acidula J.R. Forst. \& G. Forst.

Sulawesi distribution - North, Central, South

Collection(s) - Alston 16039; bb 16993, 17048; Kaudern 432; Snellius-II 11292A, 11293A, $11635 \mathrm{~A}$

\section{MAGNOLIACEAE}

\section{ELMERILLIA}

Elmerrillia ovalis (Miq.) Dandy

Vernacular name(s) - Fahas; Faäs; Kayu Campaka [Kotamobagu]; Oeroe; Woeloe; Woeroe

Sulawesi distribution - North, Central, South, South-East

Altitude - 0-1200 m

Collection(s) - Afriastini 2112; Alston 15973; bb 12386, 13487, 13512, 15701, 19979, 19980, 24510, 31815, 31863, 32323, 32459, 32472; Burley, Partomihardjo \& Rachman 3618, 3930; Cel III 00, III 8, III 101; De Vogel \& Vermeulen 6811, 7054, 7064; De Vriese \& Teijsmann 25; Forman 202; Koorders 17779, 17787, 17788, 17790; Meijer 9611, 9726; Okada 3379; Tantra 1509; Van Balgooy 3430 
EImerrillia tsiampacca (L.) Dandy var. tsiampacca

Vernacular name(s) - Champaka Kuning; Wasian

Sulawesi distribution -- North, Central, South Altitude - 200-1300 m

Collection(s) - Alston 16539; bb 19726, 20000 , 21987, 21988, 21989, 24169, 28241, 32567. 32803, 33115; Koorders 17770, 17772, 17776, 17777, 17778; Meijer 11006

\section{MAGNOLIA}

Magnolia candollii (Blume) H. Keng var. candollii

Vernacular name(s) - Hamoru [Besoa]; Tikao Sulawesi distribution - North, Central, South, South-East

Altitude - 0-2000 m

Collection(s) - bb 24079, 31912, 32568; Burley, Partomihardjo \& Rachman 3576, 3837, 3864; De Vogel 2531, 5176; De Vogel \& Vermeulen 6619, 6726; Forsten 355; Koorders 17785, 17792; Meijer 9360, 11304; Noerkas 257; Prawiroatmodjo \& Maskuri 1310; Prawiroatmodjo \& Soewoko 1707, 1961; Ramlanto 227; Tantra 1546, 1603; Whitmore \& Sidiyasa 3398

Magnolia phaulantha Dandy ex Noot.

Sulawesi distribution - South

Altitude $-1600 \mathrm{~m}$

Collection(s) - bb 29194

Magnolia villosa (Miq.) H. Keng

Sulawesi distribution - North

Altitude $-150 \mathrm{~m}$

Collection(s) - Burley, Partomihardjo \& Rach$\operatorname{man} 4080$

\section{MANGLIETIA}

Manglietia glauca Blume var. glauca

Sulawesi distribution - North, South

Altitude - 1000-1500 m

Collection(s) - bb 19703, 20908; Meijer 10010,10080
MICHELIA

\section{Michelia champaca L. var. champaca}

Vernacular name(s) - Kananga (usually for Cananga odorata, Annonaceae!)

Sulawesi distribution - South

Collection(s) - Bünnemeyer 12581; De Vriese \& Teijsmann 131; Rachmat 266

\section{MALPIGHIACEAE}

\section{ASPIDOPTERIS}

Aspidopterys celebensis Arènes

Sulawesi distribution - North

Altitude - 0-350 m

Collection(s) - Burley, Partomihardjo \& Rachman 3552; Koorders 19642

\section{HIPTAGE}

Hiptage benghalensis (L.) Kurz

Sulawesi distribution - South

Altitude $\longrightarrow 750 \mathrm{~m}$

Collection(s) - Bünnemeyer 11522; Meijer 10826; Rachmat 873; Teijsmann HB 11729, HB 11790, HB 11829, HB 11832, HB 12116, HB 12530

Hiptage luzonica Merr.

Sulawesi distribution - North

Collection(s) - J.J. Smith 633, 635

\section{RYSSOPTERIS}

Ryssopterys timoriensis (DC.) Juss.

Vernacular name(s) - Loka-loka

Sulawesi distribution - South-East

Altitude $-180 \mathrm{~m}$

Collection(s) - Kjellberg 301; Rachmat 165

\section{MALVACEAE}

\section{ABELMOSCHUS}

Abelmoschus manihot (L.) L.W. Medicus forma leptodactylus Bakh.

Sulawesi distribution - North

Collection(s) - Koorders 17802 
Abelmoschus manihot (L.) L.W. Medicus subsp. manihot

Sulawesi distribution - North

Collection(s) - Koorders 17810

Abelmoschus moschatus L.W. Medicus subsp. moschatus

Sulawesi distribution - North, Central, South Altitude - 600-1000 m

Collection(s) - Eyma 1211; Kaudern 69, 394; Noerkas 59, 60; Rachmat 213

Abelmoschus spec.

Sulawesi distribution - North, South-East

Altitude $-250 \mathrm{~m}$

Collection(s) - Whitmore \& Sidiyasa TCW 3377; Widjaja 761

\section{ABUTILON}

Abutilon crispum (L.) L.W. Medicus

Sulawesi distribution - South

Collection(s) - Yoshida 892

Abutilon indicum (L.) Sweet

Sulawesi distribution - Central

Lore Lindu National Park \& environs - present Altitude - $400 \mathrm{~m}$

Collection(s) - Meijer 10163

Abutilon indicum (L.) Sweet subsp. albescens (Miq.) Borss.Waalk.

Sulawesi distribution - North, Central, South, South-East

Altitude - 0-60 m

Collection(s) - Elbert 2541; Eyma 4198;

Kaudern 497; Rachmat 332

Abutilon indicum (L.) Sweet subsp. indicum

Sulawesi distribution - Central

Lore Lindu National Park \& environs - present Altitude - $30 \mathrm{~m}$

Collection(s) - Van Balgooy 2986

Abutilon indicum (L.) Sweet var. australiense Hochr.

Sulawesi distribution - Central

Collection(s) - Kaudern 497
Abutilon spec.

Sulawesi distribution - South-East

Collection(s) — Widjaja 613

\section{GOSSYPIUM}

Gossypium arboreum L. var. obtusifolium (Roxb.) Mart.

Sulawesi distribution - North, South-East

Altitude - 0-125 m

Collection(s) - Elbert 2527, 2906, 2951; Rachmat 363; Van Vuuren 390

Gossypium barbadense L. var. acuminatum (Roxb.) Mart.

Vernacular name(s) - Kapasa

Sulawesi distribution - North, South-East

Altitude $-0-200 \mathrm{~m}$

Collection(s) - Elbert 3285; Rachmat 26

Gossypium hirtum L. var. hirtum

Sulawesi distribution - South-East

Altitude - 0-200 m

Collection(s) - Elbert 3365

\section{Gossypium hirtum L.}

Sulawesi distribution - Central

Lore Lindu National Park \& environs - present Altitude - $400 \mathrm{~m}$

Collection(s) - Meijer 10162

\section{HIBISCUS}

Hibiscus decaspermus Koord. \& Valeton

Sulawesi distribution - South-East

Collection(s) — Forsten 447; Kjellberg 1248

Hibiscus lunariifolius Willd.

Sulawesi distribution - South-East

Altitude $-400 \mathrm{~m}$

Collection(s) - Bünnemeyer 11752

Hibiscus panduriformis Burm.f.

Sulawesi distribution - South

Collection(s) - Noerkas 73

Hibiscus surattensis L.

Vernacular name(s) - Karik karik; Karmei; Liloepang hodo 
Sulawesi distribution - North, Central, South, South-East

Altitude - 0-750 m

Collection(s) - Bünnemeyer 10622, 10766, 11432, 11747, 11790; Elbert 2667, 2678; Koorders 17811; Noerkas 264; Rachmat 358

\section{Hibiscus tiliaceus L.}

Sulawesi distribution - North, South, SouthEast

Altitude - 20-400 m

Collection(s) - De Vogel 5869; Prawiroatmodjo \& Soewoko 1860

Hibiscus tiliaceus L. subsp. tiliaceus

Vernacular name(s) - Baru; Doekoe doekoe; Kajoe baroe; Waroe

Sulawesi distribution - North, Central, South, South-East

Altitude - 0-1000 m

Collection(s) - Amir 7; Bloembergen 4211; Bünnemeyer 11521, 12511; Cel V 243, V 394; Elbert 2809, 2839, 2877; Koorders 17821, 17822; Lam 3029; Meijer 10123; Rachmat 164, 174, 812

Hibiscus tiliaceus L. subsp. celebicus (Koord.) Borss.Waalk.

Sulawesi distribution - North

Altitude $-50 \mathrm{~m}$

Collection(s) - Koorders 17816, 17819, 17820, 17823

\section{Hibiscus vitifolius $\mathrm{L}$.}

Sulawesi distribution - South

Altitude $-50 \mathrm{~m}$

Collection(s) - Kjellberg 1393; Teijsmann HB 13938

\section{Hibiscus spec.}

Sulawesi distribution - South

Collection(s) - Chin 3506, 3563, 3589

\section{THESPESIA}

Thespesia lampas (Cav.) Dalzell \& A. Gibson Vernacular name(s) - Kapasa; Poeroe poeroe Sulawesi distribution - South, South-East Altitude $-50-280 \mathrm{~m}$

Collection(s) - Bünnemeyer 10565, 10779; Rachmat 8, 19; Widjaja 705
Thespesia populnea (L.) Sol. ex Corrêa

Sulawesi distribution - South-East Altitude $-5 \mathrm{~m}$

Collection(s) — bb 21794; Rachmat 811

\section{MELASTOMATACEAE}

ASTRONIA

Astronia klabatensis J.F. Maxwell

Sulawesi distribution - North

Altitude $-500 \mathrm{~m}$

Collection(s) - Forman 256

Astronia macrophylla Blume

Vernacular name(s) - Tengtaramisián; Tentaramisian

Sulawesi distribution - North

Altitude $-50-800 \mathrm{~m}$

Collection(s) - bb 13764, 18681; Burley, Partomihardjo \& Rachman 3685; Koorders 17845, 17846, 17849; Lam 2695, 2979, 3320

\section{Astronia spectabilis Blume}

Sulawesi distribution - South

Collection(s) - Teijsmann HB 13696, HB 13713

Astronia stapfii Koord.

Sulawesi distribution - North

Altitude $-700 \mathrm{~m}$

Collection(s) - Koorders 17851

Astronia spec.

Vernacular name(s) - Pampeda [Besoa]

Sulawesi distribution - North, Central, South Lore Lindu National Park \& environs - present Altitude - 250-2300 m

Collection(s) - bb 26642; De Vogel 5266, 5450, 5558; Koorders 17848; Tantra 1583; Van Balgooy 3768; Whitmore \& Sidiyasa TCW 3492

\section{MEDINILLA}

\section{Medinilla myrtiformis Triana}

Sulawesi distribution - North

Altitude $-1200 \mathrm{~m}$

Collection(s) - Koorders 17881 
Medinilla teysmanni Miq.

Sulawesi distribution - North

Collection(s) - Teijsmann HB 5277

\section{MELASTOMA}

Melastoma horridum Bakh.f.

Sulawesi distribution - South

Altitude - $1350 \mathrm{~m}$

Collection(s) - Bünnemeyer 11596,

\section{MEMECYLON}

Memecylon celebicum Bakh.f.

Sulawesi distribution - South

Altitude $-5 \mathrm{~m}$

Collection(s) - bb 19711

Memecylon crassifolium Bakh.f.

Sulawesi distribution - South

Altitude $-100 \mathrm{~m}$

Collection(s) - Cel 452

\section{Memecylon paniculatum Jack}

Vernacular name(s) - Goroedoewana; Kondale Sulawesi distribution - North, South, SouthEast

Altitude - 50-1250 m

Collection(s) - bb 9709, 24497, 28257; De Vogel 2458, 2486; Koorders 17885, 17886, 18268, 18275, 19581; Prawiroatmodjo \& Maskuri 1448, 1453, 1498

\section{Memecylon spec.}

Vernacular name(s) - Manito [Besoa]

Sulawesi distribution - North, Central, South, South-East

Altitude $-20-1000 \mathrm{~m}$

Collection(s) - De Vogel 6086, 6262, 6338, 6372; Johansson 398; Koorders 17884; Prawiroatmodjo \& Soewoko 1935; Ramlanto 195; Tantra 1532; Van Balgooy 3699, 3756, 3830, 3851, 4060; Whitmore \& Sidiyasa TCW 3473

\section{OSBECKIA}

\section{Osbeckia dolichophyla Naudin}

Sulawesi distribution - South Altitude - 0-1400 m
Collection(s) - Bünnemeyer 11595; Zollinger 3302

\section{PTERNANDRA}

\section{Pternandra coerulescens Jack}

Vernacular name(s) - Paka Paka; Tambak bate Sulawesi distribution - North, Central, South, South-East

Altitude - 15-150 m

Collection(s) - bb 21607; Cel III 24; Eyma 3399, 4020; Prawiroatmodjo \& Maskuri 1109,1112

Pternandra gracilis (Cogn.) M.P. Nayar

Sulawesi distribution - South

Collection(s) - Meijer 10964

\section{Pternandra spec.}

Sulawesi distribution - Central, South, SouthEast

Altitude - 20-1100 m

Collection(s) - Amir 138; Coode 6081, 6119; Prawiroatmodjo \& Soewoko 1909, 1923; Van Balgooy 3849

\section{MELIACEAE}

\section{AGLAIA}

\section{Aglaia argentea Blume}

Vernacular name(s) - Alo [Besoa]; Lasa dondri Sulawesi distribution - North, Central, South, South-East

Altitude - 50-1500 m

Collection(s) - Cel I 24, I 24a; Coode 6212; De Vogel 5532, 5927; De Vogel \& Vermeulen 6562; De Vriese \& Teijsmann 102; Koorders $17889,17890,17894,17905,17912,17914$, 17915; Meijer 10012

\section{Aglaia edulis A. Gray}

Sulawesi distribution - North

Collection(s) - Koorders 17918

Aglaia elaeagnoidea (A. Juss.) Benth.

Sulawesi distribution - North

Altitude - 3-900 m

Collection(s) - Alston 16097; bb 16984;

Koorders 17899; Van Balgooy 2975 


\section{Aglaia elliptica Blume}

Sulawesi distribution - North, Central, South, South-East

Altitude - 0-270 m

Collection(s) - Alston 16044; bb 19432, 21360; Cel V 246; Chin 3510; De Vogel 2554, 2593; De Vogel \& Vermeulen 6569, 6605; De Vriese 68; Eyma 3718; Forsten 460; Koorders 17888, 17891, 17902, 17916, 17934, 17938; Meijer 10730; Prawiroatmodjo \& Maskuri 1544; Whitmore \& Sidiyasa TCW 3497 , TCW 3527

\section{Aglaia eximia Miq.}

Sulawesi distribution - Central, South

Lore Lindu National Park \& environs - present

Collection(s) - Eyma 3716; Teijsmann HB 13854

Aglaia grandis Korth. ex Miq.

Sulawesi distribution - North

Altitude - 0-500 m

Collection(s) 一 Koorders 17892, 17900, 17908, 17929

\section{Aglaia korthalsii Miq.}

Vernacular name(s) — La-usip [Tombulu]

Sulawesi distribution - North, Central, South Lore Lindu National Park \& environs - present Altitude - 40-1700 m

Collection(s) - Alston 15732, 16566; bb 17157, 20429, 28317, 29480; Cel V 358, V 388; De Vogel \& Vermeulen 6778; Forman 186; Johansson 205; Koorders 17909, 17921, 17923; Ramlanto 254; Tantra 1535; Van Balgooy 3132

\section{Aglaia lancilimba Merr.}

Vernacular name(s) - Woearaoe

Sulawesi distribution - North, South, SouthEast

Altitude - 1-250 m

Collection(s) - Cel III 26; Koorders 17890 , 17896, 17931; Meijer 10128; Prawiroatmodjo \& Soewoko 1705,1885

Aglaia lawii (Wight) Saldanha ex Ramamoorthy Vernacular name(s) - Kajoe djangan; Molemehoelo

Sulawesi distribution - North, South, SouthEast
Altitude - 0-200 m

Collection(s) - bb 15371, 21309, 31872, 32575; Cel I 23; Elbert 3343; Koorders 17933; Soenarko 354

Aglaia luzoniensis (Vidal) Merr. \& Rolfe

Sulawesi distribution - North, South-East

Altitude - 100-550 m

Collection(s) - De Vogel 2601; Koorders 17911, 17935, 17937; Prawiroatmodjo \& Soewoko 1904, 1956

Aglaia macrocarpa (Miq.) Pannell

Vernacular name(s) - Woloelokoe

Sulawesi distribution - South

Collection(s) - bb 11420; Cel II 490

Aglaia odoratissima Blume

Sulawesi distribution - North, Central, South Altitude - 1000-1250 m

Collection(s) - bb 28270; Hennipman 6103; Koorders 17910, 17939; Tantra 1528

Aglaia pachyphylla Koord. \& Valeton

Sulawesi distribution - South-East

Altitude - 20-250 m

Collection(s) - Prawiroatmodjo \& Soewoko 1717

\section{Aglaia palembanica Miq.}

Sulawesi distribution - Central

Lore Lindu National Park \& environs - present

Collection(s) - Eyma 3511

Aglaia silvestris Miq.

Vernacular name(s) - Galatri

Sulawesi distribution - North, South

Altitude - 5-450 m

Collection(s) - Cel I 14, III 117; De Vogel 6300; Koorders 41491

Aglaia smithii Koord.

Sulawesi distribution - North

Altitude - 200-700 m

Collection(s) - Koorders 17893, 17917

Aglaia squamulosa King

Sulawesi distribution - Central

Altitude - 250-762 
Collection(s) - Musser S 115a; Ramlanto \& Fanani 69

\section{Aglaia subcuprea Elmer}

Vernacular name(s) - Pisek

Sulawesi distribution - North

Altitude $-600 \mathrm{~m}$

Collection(s) - Forman 268

Aglaia tomentosa Teijsm. \& Binn.

Sulawesi distribution - North, Central, South Altitude - 40-1000 m

Collection(s) - bb 17164, 19613; Cel V 398; De Vogel 2553, 2579; Koorders 16531, 17919, 17930; Meijer 9588, 10950; Musser S 111a; Whitmore \& Sidiyasa TCW 3511

\section{Aglaia spec.}

Sulawesi distribution - Central

Lore Lindu National Park \& environs - present Altitude - 300-1000 m

Collection(s) - De Vogel 5173; Ramlanto \& Fanani 475

\section{APHANAMIXIS}

Aphanamixis polystachia (Wall.) R. Parker

Vernacular name(s) - Amoora; Edja; Kajoe edja; Kondongio; Linoeanoalo; Lotu [Besoa]

Sulawesi distribution - North, Central, South, South-East

Altitude - 25-1000 m

Collection(s) - bb 13760, 21776, 25002; Cel I 43, V 135, V 226; De Vogel 5170, 5187, 5189; Koorders 17962; Tantra 1561; Teijsmann HB 11871, HB 11899, HB 12323, HB 12418

\section{AZADIRACHTA}

Azadirachta excelsa (Jack) M. Jacobs

Sulawesi distribution - South-East

Collection(s) - Ham B 4

\section{CHISOCHETON}

Chisocheton celebica Koord.

Sulawesi distribution - North

Altitude $-600 \mathrm{~m}$

Collection(s) - Koorders 17950
Chisocheton ceramicus (Miq.) DC.

Sulawesi distribution - Central, South

Lore Lindu National Park \& environs - present Altitude - 750-1000 m

Collection(s) - Meijer 10028; Van Balgooy 3567

Chisocheton cumingianus (DC.) Harms

Sulawesi distribution - Central

Altitude - $304 \mathrm{~m}$

Collection(s) - Musser 573

Chisocheton cumingianus (DC.) Harms subsp. cumingianus

Vernacular name(s) - Waoetang golo; Woea tanggolo

Sulawesi distribution - South

Altitude $-250 \mathrm{~m}$

Collection(s) - Cel V 173

\section{Chisocheton koordersii Mabb.}

Sulawesi distribution - North, South-East

Altitude - 5-700 m

Collection(s) - bb 19419, 19597, 21316, 21323; Koorders 17960, 17964, 17973, 17978, 17989

\section{Chisocheton patens Blume}

Sulawesi distribution - North, South, SouthEast

Altitude - 20-600 m

Collection(s) - bb 24484; Koorders 17949; Prawiroatmodjo \& Maskuri 1109, 1307; Prawiroatmodjo \& Soewoko 1992

Chisocheton pentandrus (Blume) Merr.

Vernacular name(s) - Balola kambika; Kan ne oewak; Liouw; Loeminpoko; Mawewesar [Langowan]

Sulawesi distribution - North, South, SouthEast

Altitude $-20-2000 \mathrm{~m}$

Collection(s) - bb 6644, 12663, 13502, 14333; De Vogel 2493; Hochreutiner 146; Koorders 17986; Meijer 9690; Prawiroatmodjo \& Soewoko 1710, 1952; Teijsmann HB 11734

\section{Chisocheton warburgii Harms}

Sulawesi distribution - North

Altitude $-500 \mathrm{~m}$

Collection(s) - De Vogel 2642 


\section{CIPADESSA}

Cipadessa baccifera (Roth.) Miq.

Sulawesi distribution - Central, South, SouthEast

Altitude - 250-900 m

Collection(s) - Bünnemeyer 12440; Chin 3653; Meijer 10195; Van Balgooy 3621

\section{DYSOXYLUM}

\section{Dysoxylum alliaceum (Blume) Blume}

Vernacular name(s) - Kajoe lepaena

Sulawesi distribution - North, Central, South

Lore Lindu National Park \& environs - present

Altitude - 15-1800 m

Collection(s) - bb 19814, 22076; De Vogel 5445; Koorders 17972; Prawiroatmodjo \& Soewoko 1945; Teijsmann HB 13678; Van Balgooy 3090

\section{Dysoxylum arborescens (Blume) Miq.}

Vernacular name(s) - Wandiboka poete/Talo Sulawesi distribution - North, South

Altitude - 60-400 m

Collection(s) - bb 18805; Cel I 58, V 211; Forsten 166, 348; Hose 781; Koorders 17894, 17983, 17985, 17987; Riedel 158

\section{Dysoxylum cyrtobotryum Miq.}

Sulawesi distribution - North

Altitude - 100-500 m

Collection(s) - Forman 524; Koorders 5027, 17904, 17956

\section{Dysoxylum densiflorum Miq.}

Vernacular name(s) - Toembawa roudor

Sulawesi distribution - North, Central, South

Altitude $-200-1000 \mathrm{~m}$

Collection(s) - bb 13762, 32802; De Vogel 5573; Koorders 17931, 17953, 17954, 17963

Dysoxylum excelsum Blume

Sulawesi distribution - Central

Lore Lindu National Park \& environs — present Altitude $-1700 \mathrm{~m}$

Collection(s) - Van Balgooy 3382, 3387
Dysoxylum gaudichaudianum (A. Juss.) Miq.

Sulawesi distribution - North, Central, South

Collection(s) - De Vriese \& Teijsmann 154; Koorders 17966; Meijer 9417; Teijsmann HB 13859

\section{Dysoxylum macrocarpum Blume}

Sulawesi distribution - North, Central

Altitude - 121-1200 m

Collection(s) - bb 17685; Koorders 17970; Musser 778

Dysoxylum mollissimum Blume subsp. molle (Miq.) Mabb.

Sulawesi distribution - North, Central, South, South-East

Altitude - 20-1800 m

Collection(s) - bb 17969, 20274, 21714, 23897; De Vogel 5246; Koorders 17969, 17976, 17979; Tantra 1510

Dysoxylum nutans (Blume) Miq.

Vernacular name(s) - Lansa bangi; Leleuse; Lonca ibo [Besoa/Kulawi]

Sulawesi distribution - North, Central, South, South-East

Altitude - 0-1200 m

Collection(s) - Bünnemeyer 11455; Burley, Partomihardjo \& Rachman 4094; Cel V 124; Darnaedi 1499; De Vogel 5542, 5631; Elbert 2970, 3007, 3067, 3219; Johansson 72, 190; Meijer 9709, 9946, 9996, 10884; Ramlanto 260; Ramlanto \& Fanani 605; Tantra 1540, 1559; Teijsmann HB 12414; Van Balgooy 3004, 3135, 3417, 3590; Widjaja 672

Dysoxylum parasiticum (Osbeck) Kosterm.

Vernacular name(s) - Kondongio poete; Lingkoboe poete

Sulawesi distribution - North, South

Altitude - 300-1060 m

Collection(s) - bb 17122; Cel V 203

\section{Dysoxylum spec.}

Sulawesi distribution - North, Central

Altitude - 220-1750 m

Collection(s) - bb 19425; De Vogel 5245, 5464; De Vogel \& Vermeulen 6781; Forman 252; Ramlanto \& Fanani 470 


\section{LANSIUM}

\section{Lansium domesticum Jack}

Vernacular name(s) - Lansa takau

Sulawesi distribution - North, South, SouthEast

Altitude - 25-300 m

Collection(s) - bb 22062; Cel IV 173, IV 174, IV 176, V 235; De Vogel \& Vermeulen 6699; Koorders 19703

\section{MELIA}

Melia azedarach $\mathrm{L}$.

Sulawesi distribution - South

Collection(s) - Eyma 1088

\section{PSEUDOCLAUSENA}

Pseudoclausena chrysogyne (Miq.) T.P. Clark

Sulawesi distribution - South, South-East

Altitude $-0-300 \mathrm{~m}$

Collection(s) - Elbert 3188, 3225; Prawiroatmodjo \& Soewoko 1691, 1962

\section{REINWARDTIODENDRON}

\section{Reinwardtiodendron celebicum Koord.}

Sulawesi distribution - North

Collection(s) - Koorders 19713

Reinwardtiodendron humile (Hassk.) Mabb.

Sulawesi distribution - South-East

Altitude - 100-300 m

Collection(s) - Prawiroatmodjo \& Maskuri 1216, 1436; Prawiroatmodjo \& Soewoko 1911,1937

\section{SANDORICUM}

Sandoricum koetjape (Burm.f.) Merr.

Sulawesi distribution - North, Central, South, South-East

Altitude - 5-300 m

Collection(s) - bb 19433, 19595, 20900, 21380, 22322, 28721, 31820, 33102; Cel V 281; Coode 6061; Koorders 19704, 19705
Sandoricum spec.

Sulawesi distribution - South-East

Altitude $-50 \mathrm{~m}$

Collection(s) - Prawiroatmodjo \& Maskuri 1305

\section{TOONA}

Toona sureni (Blume) Merr.

Vernacular name(s) - Molopoga

Sulawesi distribution - North, Central, South Altitude - 200-800 m

Collection(s) - bb 15030, 22592, 25555, 31481; Cel V 273; Koorders 17943, 17944, 17945,17947

\section{TURRAEA}

Turraea pubescens Hell.

Sulawesi distribution - Central

Lore Lindu National Park \& environs - present Altitude - $200 \mathrm{~m}$

Collection(s) - De Vogel 5028

WALSURA

\section{Walsura brachybotrys Merr.}

Sulawesi distribution - Central, South-East Altitude - 50-60 m

Collection(s) - Musser 77; Prawiroatmodjo \& Maskuri 1275

\section{XYLOCARPUS}

Xylocarpus granatum K.D. Koenig

Sulawesi distribution - North, South

Altitude - 0-2 m

Collection(s) - Cel III 73, III 95; Koorders 17940; Ramlanto 148

\section{Xylocarpus mekongensis Pierre}

Vernacular name(s) - Antai molosigo; Kaong tjoeke; Tamboe

Sulawesi distribution - North, South, SouthEast

Altitude - 0-10 m

Collection(s) - bb 3028, 14444, 17061, 21760, 32325, 32327, 32524; Elbert 3241; Rachmat 791 
Xylocarpus rumphii (Kostel.) Mabb.

Sulawesi distribution - North

Collection(s) - bb 17049; Koorders 17941, 17942; Rachmat 650

\section{MENISPERMACEAE}

\section{ARCANGELISIA}

Arcangelisia flava (L.) Merr.

Vernacular name(s) - Akar kuning [Bahasa Indonesia]; Wala Kuning [Kolawi]

Sulawesi distribution - Central, South

Lore Lindu National Park \& environs - present

Altitude - 500-700 m

Collection(s) - De Vogel 5628; Hennipman 5808; Johansson 196; Meijer 10861

\section{CISSAMPELOS}

\section{Cissampelos pareira $\mathrm{L}$.}

Vernacular name(s) - Boelane [Alf.]

Sulawesi distribution - Central, South

Lore Lindu National Park \& environs - present

Collection(s) - Eyma 3504; Rant 891

\section{CYCLEA}

\section{Cyclea cauliflora Merr.}

Sulawesi distribution - Central, South

Altitude $-5-400 \mathrm{~m}$

Collection(s) - De Vogel 5919; Van Balgooy 3587

\section{DIPLOCLISIA}

Diploclisia glaucescens (Blume) Diels

Sulawesi distribution - South-East

Altitude - 20-250 m

Collection(s) - Prawiroatmodjo \& Soewoko 1661

\section{HAEMATOCARPUS}

\section{Haematocarpus subpeltatus Merr.}

Sulawesi distribution - North

Altitude $-0-500 \mathrm{~m}$

Collection(s) - Koorders 17991; Prawiroatmodjo \& Soewoko 1843

\section{HYPSERPA}

\section{Hypserpa nitida Miers}

Sulawesi distribution - Central, South, SouthEast

Altitude - 70-1000 m

Collection(s) - Amir 94; Chin 3634; De Vogel 5059, 5860, 5921; Van Balgooy 2961

\section{PACHYGONE}

Pachygone ovata (Poir.) Hook.f. \& Thomson

Sulawesi distribution - South

Altitude - $100 \mathrm{~m}$

Collection(s) - Van Balgooy 3815

\section{PERICAMPYLUS}

Pericampylus glaucus (Lam.) Merr.

Vernacular name(s) - Talimba-as

Sulawesi distribution - North, Central, South

Lore Lindu National Park \& environs - present

Altitude - 0-1200 m

Collection(s) - De Vogel 5958; Elbert 3367; Kjellberg 1310; Koorders 17999, 18000, 18105, 18106; Lam 2541; Meijer 9705, 10230; Prawiroatmodjo \& Soewoko 1830

\section{PYCNARRHENA}

\section{Pycnarrhena tumefacta Miers}

Sulawesi distribution - North, South

Altitude - $250 \mathrm{~m}$

Collection(s) - Teijsmann HB 12857; Whitmore \& Sidiyasa TCW 3474

\section{STEPHANIA}

\section{Stephania corymbosa Miq.}

Sulawesi distribution - North

Altitude - 50-220 m

Collection(s) - De Vogel \& Vermeulen 6522; Koorders 17998, 18102

Stephania japonica (Thunb.) Miers

Sulawesi distribution — South

Altitude $-50 \mathrm{~m}$

Collection(s) - Bünnemeyer 10656; Yoshida 865 
Stephania japonica (Thunb.) Miers var. discolor (Miq.) Forman

Sulawesi distribution - South

Altitude $-50 \mathrm{~m}$

Collection(s) - Afriastini 2143

\section{TINOSPORA}

Tinospora glabra Merr.

Sulawesi distribution - South, South-East Altitude - 0-400 m

Collection(s) — De Vogel 5910; Elbert 3356

\section{MONIMIACEAE}

KIBARA

Kibara coriacea (Blume) Tul.

Sulawesi distribution - North, Central, South, South-East

Altitude - 20-1500 m

Collection(s) - Koorders 16015, 18114, 18117, 18119; Meijer 10017; Noerkas 439; Prawiroatmodjo \& Soewoko 1877; Van Balgooy 3013

\section{MORACEAE}

\section{ANTIARIS}

\section{Antiaris toxicaria Lesch.}

Vernacular name(s) - Ipo poete

Sulawesi distribution - North, South

Altitude - 50-900 m

Collection(s) - Cel V 150; Koorders 19038, 19167, 19245, 19375, 19376; Meijer 10842, 10867, 10880; Teijsmann HB 12751

\section{ARTOCARPUS}

Artocarpus communis J.R. Forst. \& G. Forst.

Vernacular name(s) - tipuru

Sulawesi distribution - North, South

Altitude $-250 \mathrm{~m}$

Collection(s) — Lam 2895; Meijer 11236

\section{Artocarpus fretissii Hassk.}

Vernacular name(s) - iloei bobi rongo; Kajoe Maumbij; Kelembie; koeli bobi; linka uwing'a; Rololi
Sulawesi distribution — North, Central, South, South-East

Altitude - 0-1000 m

Collection(s) - bb 5573, 6041, 18022, 21754, 23909, 28212, 32510; Cel 216, I 19, III 55; De Vriese \& Teijsmann 38; Koorders 19045, 19048, 19052, 19054, 19055, 19058, 19059, 19060, 19061, 19185, 19312; Lam 2626; Riedel HB 5841; Tantra 1549; Teijsmann HB 5789, HB 12359

\section{Artocarpus heterophyllus Lam.}

Sulawesi distribution - North, South-East

Altitude $-0-200 \mathrm{~m}$

Collection(s) - Elbert 3324

Artocarpus integer (Thunb.) Merr.

Sulawesi distribution - South, South-East

Altitude - 15-100 m

Collection(s) - bb 13550, 24509, 217531; Cel III 127

\section{Artocarpus reticulatus Miq.}

Sulawesi distribution - North, Central, South Altitude - 50-1000 m

Collection(s) - Cel V 228; Koorders 19046, 19047, 19049, 19051, 19053; Meijer 10738, 10907; Tantra 1536

\section{Artocarpus sericicarpus Jarrett}

Vernacular name(s) - awa'a

Sulawesi distribution - North, Central, South Altitude - 20-100 m

Collection(s) - bb 22985, 28729, 32458; Cel II 81; Lam 2845

\section{Artocarpus teysmannii Miq.}

Vernacular name(s) - Gotifoeloe; Tipoeloe Sulawesi distribution - North, Central, South, South-East

Altitude - 1-750 m

Collection(s) - bb 4988, 21110, 28240, 28738, $32359,32545,32607$; Cel III 69, III 87, III 88; Noerkas 270, 443, 456

\section{Artocarpus spec.}

Sulawesi distribution - North, South-East Altitude - 20-300 m

Collection(s) - De Vogel \& Vermeulen 6603, 6927; Prawiroatmodjo \& Soewoko 1898, 1918 


\section{BROUSSONETIA}

Broussonetia luzonica (Blanco) Bur. var. glabra (Warb.) Corner

Sulawesi distribution - North, Central, SouthEast

Altitude $-50-200 \mathrm{~m}$

Collection(s) - bb 19435, 21759; Kaudern 478

Broussonetia papyrifera Vent.

Vernacular name(s) - Boejang-boejang

Sulawesi distribution — North, Central, South, South-East

Altitude - 5-200 m

Collection(s) - bb 21717, 29472; Cel I 47, III 64, III 159, V 350; Kaudern 436; Kjellberg 2874

Broussonetia papyrifera Vent. var. integrifolia Miq.

Sulawesi distribution - North

Collection(s) - Teijsmann HB 7319

FATOUA

Fatoua pilosa Gaudich.

Vernacular name(s) - bangelo; marulum'a

Sulawesi distribution - North, South, SouthEast

Altitude - 0-60 m

Collection(s) - Elbert 2571; Kjellberg 20; Lam 3195; Teijsmann HB 12874, HB 13920

FICUS

Ficus adenosperma Miq.

Sulawesi distribution - North

Collection(s) - Teijsmann HB 5363, HB 5853

Ficus ampelos Burm.f.

Vernacular name(s) - Popi

Sulawesi distribution - North, Central, South, South-East

Altitude - 0-900 m

Collection(s) - bb 21352; De Vogel 5672; De Vogel \& Vermeulen 6695, 6724, 6919; Elbert 2746, 3385; Forman 200; Koorders 19118, 19156, 19166, 19353, 19358; Noerkas 504; Prawiroatmodjo \& Maskuri 1072; Teijsmann HB 12625; Yoshida 1216
Ficus annulata Blume

Sulawesi distribution - North, South, SouthEast

Altitude $-0-880 \mathrm{~m}$

Collection(s) - bb 18125; Elbert 2748, 3375; Koorders 19106; Meijer 10918; Prawiroatmodjo \& Maskuri 1193

\section{Ficus benjamina $\mathrm{L}$.}

Vernacular name(s) - Baringing [Tonsea]

Sulawesi distribution - North, South

Collection(s) - Alston 16085; Cel IV 115; Noerkas 430; Teijsmann HB 12684, HB 12701, HB 13864

Ficus botryocarpa Miq.

Sulawesi distribution - North

Collection(s) - Teijsmann HB 5261

Ficus callosa Willd.

Sulawesi distribution - North, South

Collection(s) - Koorders 19130, 19217; Meijer 10687

Ficus cauta Corner

Sulawesi distribution - North

Altitude - 600-1200 m

Collection(s) - bb 13517; Forman 350; Koorders 19255

Ficus chrysolepis Miq.

Sulawesi distribution - North

Collection(s) - Teijsmann HB 5310

Ficus congesta Roxb.

Sulawesi distribution - North

Collection(s) - Teijsmann HB 5850

Ficus copiosa Steud.

Sulawesi distribution - North

Altitude $-50-700 \mathrm{~m}$

Collection(s) - Koorders 19195, 19201, 19305 , 19319, 19356

Ficus crassiramea Miq.

Vernacular name(s) - nunu'a

Sulawesi distribution - North

Altitude - 1-150 m 
Collection(s) - Koorders 19126, 19160; Lam 2488, 2941; Ramlanto \& Fanani 707

Ficus deltoidea Jack var. deltoidea

Sulawesi distribution - South

Altitude $-400 \mathrm{~m}$

Collection(s) - Chin 3436; De Vogel 5789

Ficus erythrosperma Miq.

Sulawesi distribution - North, Central, South, South-East

Altitude - 0-1000 m

Collection(s) - bb 22061; Cel V 162, V 163; De Vogel \& Vermeulen 6884; Hennipman 5647; Koorders 19198, 19204, 19207, 19230, 19261, 19270; Riedel 5814; Van Balgooy 3919

Ficus fulva Reinw. ex Blume

Sulawesi distribution - North, South

Altitude - 25-850 m

Collection(s) - Cel IV 101; Hennipman 5976; Koorders 19137, 19139, 19140; Yoshida 1114; Yusuf \& Wahyono 132

Ficus geocarpa Miq.

Sulawesi distribution — North

Collection(s) - Teijsmann HB 5364

Ficus gibbosa Blume

Sulawesi distribution - North, South

Altitude $-10 \mathrm{~m}$

Collection(s) - Lam 2450; Teijsmann HB 13793

Ficus glomerata Roxb. var. elongata King

Vernacular name(s) - Miraeng

Sulawesi distribution - North, South

Altitude $-280-700 \mathrm{~m}$

Collection(s) - Bünnemeyer 10778, 10780; Koorders 19141, 19350

Ficus gul K. Schum. \& Lauterb.

Vernacular name(s) - Ambira

Sulawesi distribution - North, Central, South, South-East

Altitude - 0-1000 m

Collection(s) - Alston 16490; De Joncheere 1077; De Vogel 5051; De Vogel \& Vermeulen 6566; Elbert 2846, 8331; Koorders 19123,
19191, 19202, 19208, 19239, 19241, 19276; Lam 2585; Van Balgooy 3596; Yusuf \& Wahyono 42

Ficus heteropleura Blume

Vernacular name(s) - Kajoe ara; Keroe keroe Sulawesi distribution - North, Central

Altitude - 700-1200 m

Collection(s) - Bünnemeyer 11322, 11660; Koorders 19205, 19213, 19259, 19270

\section{Ficus hispida L.}

Sulawesi distribution - South-East

Altitude $-0-200 \mathrm{~m}$

Collection(s) - Elbert 2978, 3290

Ficus irisana Elmer

Sulawesi distribution - North

Collection(s) - Teijsmann HB 5769

Ficus lepicarpa Blume

Sulawesi distribution - North, South

Altitude - 1000-1100 m

Collection(s) - Hennipman 6099; Koorders $19120,19354,19357$

Ficus microcarpa L.f.

Sulawesi distribution - Central

Lore Lindu National Park \& environs - present Altitude $-200 \mathrm{~m}$

Collection(s) - Meijer 10239

Ficus minahassae Miq.

Vernacular name(s) - Kekewang, Werenkusai [Tonsea, Tombulu]; rinit'a; Toeloeponoe; Weren Kusei [Tombulu]

Sulawesi distribution - North

Altitude - 10-700 m

Collection(s) - Alston 16186; bb 13806; Forman 285, 286; Koorders 19143, 19144, 19177 , 19229, 19256, 19741; Lam 2733; Whitmore \& Sidiyasa 3386

Ficus obscura Blume var. borneensis (Miq.) Corner

Sulawesi distribution - North

Altitude - $100 \mathrm{~m}$

Collection(s) - Afriastini 2021 
Ficus oleaefolia King

Sulawesi distribution - South

Altitude $-1700 \mathrm{~m}$

Collection(s) - Teijsmann HB 227

Ficus pilosa Reinw. ex Blume

Vernacular name(s) - Noenoe molaba Apalim; Noenoe poeho; Noenoemolaba

Sulawesi distribution - North, South, SouthEast

Altitude - 5-300 m

Collection(s) - bb 21596; Cel V 151, V 158; Koorders 19338

Ficus schwarzii Koord.

Vernacular name(s) - Are nasa; Arinasa; Lambere

Sulawesi distribution - North, Central, South

Altitude - 900-2000 m

Collection(s) - Bünnemeyer 11168, 12056, 12059; Eyma 1710; Koorders 1919, 19252

Ficus septica Rumph. ex Burm.

Vernacular name(s) - buas; Loloyan [Tombulu]; Puwah; Tobbo-tobbo; Tobo-tobo

Sulawesi distribution - North, Central, South, South-East

Altitude $-0-1000 \mathrm{~m}$

Collection(s) - Alston 16271; bb 23899; Bünnemeyer 10781, 11751; Elbert 2876, 2939, 3346; Eyma 3711; Forman 203, 333; Grimes 1030; Koorders 18365, 19345; Lam 2532, 3407; Meijer 9995, 10822; Mogea 1285; Prawiroatmodjo \& Maskuri 1336

Ficus subtrinervia K. Schum. \& Lauterb. var. doormaniana (Diels) Corner

Sulawesi distribution - South-East

Collection(s) - Widjaja 733

Ficus subulata Blume

Sulawesi distribution - North, Central, South, South-East

Altitude $-0-700 \mathrm{~m}$

Collection(s) - Buwalda 3722, 3796; Elbert 3328; Koorders 19112, 19115, 19216, 19320, 24087; Ramlanto \& Fanani 610, 762; Soenarko 270

\section{Ficus tenuicaudata Corner}

Sulawesi distribution - South
Altitude $-2000 \mathrm{~m}$

Collection(s) - Eyma 1013

Ficus tinctoria G. Forst.

Sulawesi distribution - North

Collection(s) - Koorders 19327

Ficus tinctoria G. Forst. subsp. tinctoria

Vernacular name(s) - pele; tatanta

Sulawesi distribution - North, South-East

Altitude $-0-40 \mathrm{~m}$

Collection(s) - Elbert 2574; Forsten 319; Lam 3353,3452

Ficus tonsa Miq.

Sulawesi distribution - North

Collection(s) - Riedel HB 5705

Ficus variegata Blume

Vernacular name(s) - Alis [Wasopunda]; bintas'a; dopakotji

Sulawesi distribution - North, Central, South, South-East

Altitude $-0-500 \mathrm{~m}$

Collection(s) - bb 19509, 21317, 21703, 24997; Cel V 152; Coode 5852, 6020; De Vogel 5917, 5948; De Vogel \& Vermeulen 6650; Elbert 3372; Koorders 19316, 19366, 19367, 19368, 19369, 19370; Lam 2922; Meijer 10742, 10760; Noerkas 274; Prawiroatmodjo \& Soewoko 1628; Van Balgooy 3987

Ficus virgata Reinw, ex Blume

Vernacular name(s) - Peleta

Sulawesi distribution - North, South, SouthEast

Altitude - 0-1400 m

Collection(s) - bb 23901, 25539; Bünnemeyer 12641; Cel I 21; Elbert 2765; Koorders 19110, 19165, 19197, 19304, 19311, 19363; Lam 3071; Rachmat 319; Teijsmann HB 14159

Ficus spec.

Sulawesi distribution - North, Central, South, South-East

Altitude $-1100 \mathrm{~m}$

Collection(s) - Afriastini 2023, 2043; Chin 3405, 3468; De Vogel 5016, 5062, 5604, 
5903, 5907, 5942, 5943, 6229, 6239, 6242; De Vogel \& Vermeulen 6734, 6745; Hennipman 5836, 5846; Keßler et al. PK 3129, PK 3131; Koorders 19350; Prawiroatmodjo \& Soewoko 1715, 1944, 1954; Ramlanto 193; Ramlanto \& Fanani 657, 671; Soenarko 355; Van Balgooy 3682, 4044; Whitmore \& Sidiyasa 3429

\section{MACLURA}

Maclura amboinensis Blume

Sulawesi distribution - Central

Lore Lindu National Park \& environs - present Altitude $-1200 \mathrm{~m}$

Collection(s) - De Vogel 5327

Maclura cochinchinensis (Lour.) Corner

Vernacular name(s) - pundane

Sulawesi distribution - North

Altitude $-150 \mathrm{~m}$

Collection(s) - Lam 2950

\section{MALAISIA}

Malaisia scandens (Lour.) Planch.

Sulawesi distribution - South-East

Altitude - 0-250 m

Collection(s) - Elbert 2672; Kjellberg 781;

Prawiroatmodjo \& Soewoko 1768

\section{MORUS}

Morus australis Poir.

Sulawesi distribution - South

Altitude $-1050 \mathrm{~m}$

Collection(s) - bb 24504; Cel II 324, II 409; Koorders 19315; Teijsmann HB 5778

\section{STREBLUS}

\section{Streblus asper Lour.}

Vernacular name(s) - Soelè

Sulawesi distribution - North, Central, SouthEast

Lore Lindu National Park \& environs - present Altitude $-0-200 \mathrm{~m}$

Collection(s) - bb 16, 17332; Elbert 3015, 3056, 3349; Eyma 3726; Kjellberg 452; Van Balgooy 3605
Streblus elongatus (Miq.) Corner

Vernacular name(s) - Polanangka

Sulawesi distribution - South, South-East

Altitude - 20-400 m

Collection(s) — bb 8561, 22725, 22728, 23254, 23915, 26280, 32466, 32608; Cel II 116, II 433; De Vogel 6113; Meijer 11254

Streblus glaber (Merr.) Corner

Sulawesi distribution - Central, South

Altitude - 1000-1500 m

Collection(s) - bb 20269, 24201, 28313

Streblus ilicifolius (Vidal) Corner

Vernacular name(s) - Tjepe-Tjepe

Sulawesi distribution - North, Central, SouthEast

Altitude $-0-250 \mathrm{~m}$

Collection(s) - Elbert 2618; Kaudern 503; Koorders 19625; Noerkas 191; Whitmore \& Sidiyasa TCW 3432

\section{Streblus macrophyllus Blume}

Sulawesi distribution - North, Central Lore Lindu National Park \& environs - present Altitude - 0-100 m

Collection(s) - bb 17037, 17975, 29470

\section{TROPHIS}

\section{Trophis philippinensis (Burret) Corner}

Sulawesi distribution - North, South, SouthEast

Altitude - 20-800 m

Collection(s) - bb 17054, 21715, 28249, 28253;

Cel V 178, V 193; Koorders 19419, 19420; Meijer 10865

\section{MORINGACEAE}

MORINGA

\section{Moringa spec.}

Vernacular name(s) - Kayu batu [Wuasa]

Sulawesi distribution - Central

Lore Lindu National Park \& environs - present Altitude - 1100

Collection(s) - Keßler et al. PK 3126 


\section{MYRICACEAE}

MYRICA

Myrica esculenta Buch.-Ham. ex D. Don

Sulawesi distribution - South

Altitude $-2850 \mathrm{~m}$

Collection(s) - Sands 580

Myrica javanica Blume

Sulawesi distribution - North, Central

Lore Lindu National Park \& environs - present

Altitude - 1300-2450 m

Collection(s) - Darnaedi 1604; De Vogel 5364; Koorders 18120, 18121; Van Balgooy 3324

\section{MYRISTICACEAE}

ENDOCOMIA

Endocomia macrocoma (Miq.) W.J. de Wilde subsp. macrocoma

Sulawesi distribution - North

Collection(s) - Lam 2976

\section{GYMNACRANTHERA}

Gymnacranthera maliliensis R.T.A. Schouten

Sulawesi distribution - South

Altitude $-500 \mathrm{~m}$

Collection(s) - Van Balgooy 3960

Gymnacranthera spec.

Sulawesi distribution - Central

Lore Lindu National Park \& environs — present Altitude - $1040 \mathrm{~m}$

Collection(s) - KeBler et al. PK 3161

\section{HORSFIELDIA}

Horsfieldia coriacea W.J. de Wilde

Sulawesi distribution - South

Collection(s) - Cel III 27

Horsfieldia costulata (Miq.) Warb.

Sulawesi distribution - North

Collection(s) - Koorders 18156
Horsfieldia lancifolia W.J. de Wilde

Sulawesi distribution - South

Collection(s) - Cel 464

Horsfieldia parviflora (Roxb.) J. Sinclair

Sulawesi distribution - North

Altitude $-200-500 \mathrm{~m}$

Collection(s) - Koorders 18146, 18164

Horsfieldia talaudensis W.J. de Wilde

Sulawesi distribution - North

Altitude $-100 \mathrm{~m}$

Collection(s) - Lam 2628

\section{KNEMA}

Knema celebica W.J. de Wilde

Sulawesi distribution - South

Collection(s) - Cel II 318

Knema matanensis W.J. de Wilde

Sulawesi distribution - South

Altitude - $450 \mathrm{~m}$

Collection(s) - De Vogel 6060

\section{MYRISTICA}

Myristica devogelii W.J. de Wilde

Sulawesi distribution - South

Altitude $-400 \mathrm{~m}$

Collection(s) - De Vogel 6083

Myristica elliptica Hook.f. \& Thomson var. simiarum (A.DC.) Warb.

Sulawesi distribution - North

Collection(s) - Teijsmann HB 5801

\section{Myristica impressa Warb.}

Vernacular name(s) - Kadollam; Konvalo [Besoa]; Pala hutan

Sulawesi distribution - North, Central, South, South-East

Altitude - 125-1425 m

Collection(s) - bb 24131, 26017; Cel II 403; De Vogel 5288, 6369, 6397; Elbert 3025; Meijer 10061, 10793, 11016, 11021, 11273; Ramlanto \& Fanani 730 
Myristica impressinervia J. Sinclair

Sulawesi distribution - Central, South, SouthEast

Altitude $-50-800 \mathrm{~m}$

Collection(s) - Meijer 9363; Prawiroatmodjo \& Maskuri 1348, 1401; Teijsmann HB 14063

Myristica kjellbergii W.J. de Wilde

Sulawesi distribution - Central

Lore Lindu National Park \& environs - present Altitude $-700 \mathrm{~m}$

Collection(s) - Johansson 206

Myristica koordersii Warb.

Sulawesi distribution - North

Altitude - 500-1000 m

Collection(s) - De Vogel 2630; Forman 262; Koorders 18128, 18129

Myristica simiarum A.DC. subsp. celebica (Miq.) W.J. de Wilde

Sulawesi distribution - North

Altitude $-50-250 \mathrm{~m}$

Collection(s) - Koorders 18161, 18162, 18163; Whitmore \& Sidiyasa TCW 3436

Myristica ultrabasica W.J. de Wilde

Sulawesi distribution - South

Altitude $-400 \mathrm{~m}$

Collection(s) - Van Balgooy 4064

\section{MYRSINACEAE}

\section{AEGICERAS}

Aegiceras corniculatum (L.) Blanco

Sulawesi distribution - South-East

Altitude - 0-100 m

Collection(s) - Elbert 2700

Aegiceras floridum Roem. \& Schult.

Vernacular name(s) - Wolihedoe

Sulawesi distribution - North, South, SouthEast

Altitude - 0-10 m

Collection(s) - bb 15603, 17677; Elbert 3254

\section{ARDISIA}

Ardisia anaclasta B.C. Stone

Sulawesi distribution - North, Central, South Lore Lindu National Park \& environs - present Altitude - 1700-2335 m

Collection(s) - Bloembergen 3987; De Vogel 5449; Meijer 9893, 9910; Tantra 1571; Van Balgooy 3182

Ardisia celebica Scheff.

Sulawesi distribution - North

Altitude $-1200 \mathrm{~m}$

Collection(s) - De Vogel \& Vermeulen 7084, 7104; Forman 358; Koorders 18191, 18195 , 18197, 18200; Whitmore \& Sidiyasa TCW 3518

\section{Ardisia copelandii $\mathrm{Mez}$}

Vernacular name(s) - Teloha

Sulawesi distribution - Central, South

Lore Lindu National Park \& environs - present Altitude $-750 \mathrm{~m}$

Collection(s) - Cel III 15; Johansson 395

Ardisia elliptica Thunb.

Sulawesi distribution - North, Central, South, South-East

Altitude $-0-200 \mathrm{~m}$

Collection(s) - Alston 16030; Cel III 81; Elbert 3341, 3364; Forsten 343, 428; Grimes 1033, 1095; Koorders 18198; Ramlanto 212

\section{Ardisia forbesii S. Moore}

Sulawesi distribution - North, Central, South Altitude $-1000-2200 \mathrm{~m}$

Collection(s) - Bloembergen 3913, 4003; Darnaedi 1489; De Joncheere 1249; De Vogel 5157, 5440, 5548, 5610; De Vogel \& Vermeulen 7103; Hennipman 5191; Meijer 9581; Van Balgooy 3010, 3104

Ardisia forstenii Scheff.

Sulawesi distribution - North, South-East

Altitude $-0-800 \mathrm{~m}$

Collection(s) - Elbert 3297; Forsten 26; Koorders 18188

Ardisia javanica A.DC.

Sulawesi distribution - North 
Altitude - $1700 \mathrm{~m}$

Collection(s) - Milliken 1074

Ardisia lanceolata Roxb.

Sulawesi distribution - North, South

Altitude - $340 \mathrm{~m}$

Collection(s) - bb 14140; Meijer 10815

Ardisia leptalea B.C. Stone

Sulawesi distribution - South

Lore Lindu National Park \& environs - - present

Altitude - 1000-1300 m

Collection(s) - Meijer 9467

Ardisia petila B.C. Stone

Sulawesi distribution - Central, South

Lore Lindu National Park \& environs - present

Altitude - 0-1700 m

Collection(s) - Coode 5851, 6078; Meijer 9805, 9842, 11297

Ardisia pulvinulata B.C. Stone

Sulawesi distribution - Central

Lore Lindu National Park \& environs - present

Altitude - 1000-1500 m

Collection(s) - Darnaedi 1440; De Vogel 5313

Ardisia sanguinolenta Blume

Sulawesi distribution - South

Altitude -950-1100 m

Collection(s) - Bünnemeyer 11311, 11808

Ardisia sarasinii Mez

Sulawesi distribution - North, South

Altitude $-350-750 \mathrm{~m}$

Collection(s) - Burley, Partomihardjo \& Rachman 3882; Chin 3512; Milliken 939

Ardisia sumatrana Miq.

Sulawesi distribution - South

Collection(s) - Teijsmann HB 12772

Ardisia ternatensis Scheff.

Sulawesi distribution - North

Altitude - 30-1200 m

Collection(s) - Alston 16460; Burley, Partomihardjo \& Rachman 3854, 3980; De Vogel 2449, 2469; De Vriese \& Teijsmann 94; Forman 178, 234; Koorders 18189, 18202; Noerkas 322

\section{Ardisia spec.}

Sulawesi distribution - North, Central, South Altitude - 400-1500 m

Collection(s) - bb 28269; De Vogel 6129; Johansson 333; Milliken 1059

\section{DISCOCALYX}

\section{Discocalyx silverstris Holthuis}

Vernacular name(s) - Soewiny'a

Sulawesi distribution - North, South

Altitude - 70-1000 m

Collection(s) - Darnaedi 2196; Lam 2975, 3132,3166

\section{Discocalyx spec.}

Sulawesi distribution - North, South

Altitude - 250-1450 m

Collection(s) - Whitmore \& Sidiyasa TCW 3403; Wieringa 1864

\section{EMBELIA}

\section{Embelia spec.}

Vernacular name(s) - Kandis [Tontembuan]

Sulawesi distribution - North, Central, South Altitude - 310-2355 m

Collection(s) - Alston 16563; Bloembergen 3971; Bünnemeyer 10830; De Vogel 5572, 5887, 6021; Forman 307; Hennipman 6047; Meijer 9790, 9880; Milliken 958; Van Balgooy 3086, 3143, 3475; Van Steenis 10306

\section{MAESA}

\section{Maesa ramentacea Wall.}

Sulawesi distribution - South-East

Altitude - $100 \mathrm{~m}$

Collection(s) - Amir 140

\section{Maesa spec.}

Sulawesi distribution - North, Central, South, South-East

Altitude $-3800 \mathrm{~m}$

Collection(s) - Coode 5862, 6222, 6272; De Joncheere 1034; De Vogel 5037, 5311, 5544, 5814, 6016, 6039, 6221; Elbert 2840, 3472; Eyma 3800; Forman 355, 357; Hennipman 561 1; Meijer 10212; Prawiroatmodjo \& Soewoko 1732, 1734; Sidiyasa 1389 
RAPANEA

\section{Rapanea spec.}

Vernacular name(s) - Tanua [Suwawa]

Sulawesi distribution - North, Central

Altitude - 1420-2450 m

Collection(s) — De Vogel 5378A; Milliken 952

\section{MYRTACEAE}

\section{ACMENA}

Acmena acuminatissima (Blume) Merr. \& Perry

Sulawesi distribution - North, South

Altitude - 5-200 m

Collection(s) - De Vogel 2570; Lam 2841, 3451; Teijsmann HB 13726, HB 13751

\section{BAECKEA}

\section{Baeckea frutescens $\mathrm{L}$.}

Sulawesi distribution - North, Central, South, South-East

Altitude - 10-1800 m

Collection(s) - bb 20053, 24963, 30149, 31922; De Vogel 5855; Elbert 3073; Eyma 1611, 3348; Kofman 157; Meijer 11073; Ramlanto 191; Van Balgooy 3640, 4030

\section{DECASPERMUM}

Decaspermum bracteatum (Roxb.) A.J. Scott

Sulawesi distribution - North, South

Altitude - 350-750 m

Collection(s) - Burley, Partomihardjo \& Rachman 3536; Yoshida 1385

Decaspermum fruticosum J.R. Forst.

Sulawesi distribution - South

Altitude - $320 \mathrm{~m}$

Collection(s) - Ramlanto 167

Decaspermum parviflorum (Lam.) A.J. Scott

Vernacular name(s) - Parapa [Tontembuan]

Sulawesi distribution - North, Central, South Lore Lindu National Park \& environs - present Altitude - 50-1700 m

Collection(s) - Alston 15782, 16065; Bloembergen 3941; Eyma 1169, 3528, 3611; Koord- ers 18299, 18311, 18312; Lam 3273; Meijer 11040; Noerkas 214; Rachmat 647, 715, 1010

\section{Decaspermum spec.}

Sulawesi distribution - North, South

Altitude - 150-3400 m

Collection(s) - De Vogel 5815, 5820, 6015, 6377; Hennipman 6075; J.B.M. Smith 655; Van Balgooy 2966; Whitmore \& Sidiyasa 3517

\section{EUCALYPTUS}

\section{Eucalyptus deglupta Blume}

Vernacular name(s) - Kayu ingerris

Sulawesi distribution - North, Central, South Lore Lindu National Park \& environs - present Altitude - 0-1000 m

Collection(s) - bb 12384, 17000, 23907, $24137,24954,25544,28256,31904,32354$, 32437, 32444, 32618, 33080, 33106; De Vogel 5213; Whitmore \& Sidiyasa 3490

\section{EUGENIA}

\section{Eugenia beccarii Ridl.}

Sulawesi distribution - South

Altitude $-500 \mathrm{~m}$

Collection(s) - Darnaedi 1887, 1912

Eugenia calubcob C.B. Rob.

Vernacular name(s) - Mariwau

Sulawesi distribution - North

Altitude $-250 \mathrm{~m}$

Collection(s) - Lam 3165

\section{Eugenia densiflora DC.}

Sulawesi distribution - Central

Lore Lindu National Park \& environs - present Altitude $-1000 \mathrm{~m}$

Collection(s) - Meijer 9748

Eugenia polycephala Miq.

Sulawesi distribution - South, South-East

Altitude $-20 \mathrm{~m}$

Collection(s) — bb 3920; Meijer 10869, 10992

Eugenia polycephaloidea C.B. Rob.

Sulawesi distribution - South-East

Altitude $-50 \mathrm{~m}$ 
Collection(s) - Prawiroatmodjo \& Maskuri 1302

\section{Eugenia spicata Lam.}

Sulawesi distribution - North, South

Collection(s) - Meijer 11084; Rachmat 653

\section{KJELLBERGIODENDRON}

\section{Kjellbergiodendron celebicum (Koord.)} Steenis

Vernacular name(s) - jambu-jambu; Langara [Tobelais To Pado]

Sulawesi distribution - North, Central, South, South-East

Altitude - 0-900 m

Collection(s) - bb 1836, 1854, 1890, 4187, $5002,17634,18800,19646,19820,20756$, 20895, 21097, 22730, 22731, 22975, 23269, 23544, 26286, 32457, 33108; Beccari 3807; Burley et al. 3866; Cel II 240, II 241, II 242, II 243, II 334, II 353, II 485; Koorders 18094, $18319,18321,18322,18960,18964,19288$, 19289; Meijer \& Mochtar 11152, 11239, 11269, 11281, 11287; Prawiroatmodjo \& Maskuri 1303; Ramlanto 143; Ramlanto \& Fanani 683; Van Hulstijn 285; Waturandeng 812

\section{Kjellbergiodendron spec.}

Vernacular name(s) - Buah apel [Soroako]

Sulawesi distribution — North, South, SouthEast

Altitude - 50-1100 m

Collection(s) - bb 21590, 24124, 24163, 24486, 24488, 24972, 31513; Cel IV 192, IV 193; Coode 6142; De Vogel 5770, 6105, 6108, 6278, 6314; Kofman 103, 158; Van Balgooy 3778, 3882, 3951, 4023

\section{LEPTOSPERMUM}

\section{Leptospermum amboinense Blume}

Sulawesi distribution - North, Central, South Altitude - 300-2500 m

Collection(s) - bb 8574, 19823, 24125, 30148; Eyma 512, 3581; Meijer 11042, 11169

\section{Leptospermum javanicum Blume}

Vernacular name(s) - Kajoe papoea

Sulawesi distribution - North, Central, South, South-East
Altitude $-150-3300 \mathrm{~m}$

Collection(s) - bb 22860, 22861, 24207, 24962; Bünnemeyer 11860, 11897; Elbert 3128, 3487; Eyma 604, 1040, 1041, 1621, 3961; McDonald \& Ismail 3871; Monod de Froideville 231; J.B.M. Smith 681

\section{Leptospermum recurvum Hook.f.}

Sulawesi distribution - Central, South

Altitude - 2450-3400 m

Collection(s) - bb 22862; Eyma 691, 1362; Van Balgooy 3323

\section{Leptospermum spec.}

Sulawesi distribution - Central, South, SouthEast

Altitude $-400-2450 \mathrm{~m}$

Collection(s) - Coode 6196; De Vogel 5366; Kofman 273; Yusuf \& Wahyono 75

\section{METROSIDEROS}

\section{Metrosideros petiolata (Koord. \& Valeton)} Koord.

Vernacular name(s) - Iara betian; Kaloju/Langara [Bugin./Soroako]

Sulawesi distribution - North, Central, South, South-East

Altitude - 1-1100 m

Collection(s) - bb 1910, 5003, 13679, 18419, $21583,21628,21631,21636,21639,21669$, $21786,22722,22729,23264,23545,23579$, $23581,23582,23583,23584,23585,23586$, $23587,23588,23591,23835,24063,24064$, $24065,24067,24134,24485,30151,30167$, $31515,31518,31667,31668,31671,31841$, 31842, 31897, 31910; Cel II 255, II 256, II 346, II 395; De Vriese \& Teijsmann 267; Koorders 18307, 18308, 18309; Meijer 11174, 11280; Van Balgooy 3701, 3772, 3796, 3800, 4046, 4084; Widjaja 488

Metrosideros vera (Roxb.) Lindl.

Vernacular name(s) - Maliloe tjela; Monosi. Sulawesi distribution - North, South, SouthEast

Altitude - 50-400 m

Collection(s) - bb 18669, 19821, 31514, 31517, 31520; Cel II 346, II 384; Prawiroatmodjo \& Maskuri 1196; Ramlanto 164 


\section{Metrosideros spec.}

Vernacular name(s) - Kalodju [Timampu]

Sulawesi distribution - South

Altitude - 300-400 m

Collection(s) - De Vogel 5688, 5720, 5806, 6371

\section{PSIDIUM}

\section{Psidium guajava $\mathbf{L}$.}

Vernacular name(s) - Djamboe

Sulawesi distribution - South, South-East

Altitude - 0-1230 m

Collection(s) - Bünnemeyer 10638, 11246; De Vriese 104; Elbert 2612, 2851, 2919

\section{SYZYGIUM}

\section{Syzygium aqueum Alston}

Sulawesi distribution - North, South

Altitude - $600 \mathrm{~m}$

Collection(s) - Cel V 294; Forsten 122; Kaudern 67; Reinwardt 1011

Syzygium claviflorum (Roxb.) Wall. ex A.M. Cowan \& Cowan

Vernacular name(s) - Makor-makor

Sulawesi distribution - North

Altitude - 330-480 m

Collection(s) - Lam 3271, 3323, 3604

Syzygium cumini (L.) Skeels

Sulawesi distribution - South

Collection(s) - Robinson 2448

Syzygium samarangense (Blume) Merr. \& Perry

Sulawesi distribution - South, South-East

Altitude $-30 \mathrm{~m}$

Collection(s) - bb 4184; Yoshida 900

\section{Syzygium spec.}

Vernacular name(s) - Ajoemoito; Adjéloera; Ajamboe-Ajamboe; Anjoeroeng; Bébélé; Cenke hutan; Djambolan; Djampoeala; Dungul'a; Gampoe poeté [Lolonikaka]; Gogoon intaloen; Gora hutan [Minahassa]; Gornawar; Géné; Jambu air [Indonesia]; Kaima koelo; Kaima; Kapal; Katji-katji; Kennisfepatoe; Koempas; Koepa ilawanan; Kom- bos; Kopo [Java]; Kopé motaha, Konsioe; Korababi; Kowal intaloen; Kowal; Lara mopoete; Larra; Léléan; Maemping; Mahalansa; Makaepa; Makaimah; Makembes; Makêmbês; Makoepa; Makoepa kaunesan; Makoepa sela; Maku Wana [Besoa]; Makurole [Kolawi]; Mangkoha; Menai; Molonggaping; Nakembes; Oelaah; Pakewah; Pakowa [Tombulu]; Palehos; Papiri; Paë, kiaë; Pêngasa; Rapiri; Roko bako; Rokodéngé; Ruruhi [Kendari]; Samaki; Sapé motéa; Sio Sio lolong; Sora-Sora [Soroako/Wasuponda]; Tambe [Napu/Kulawi]; Tampesing; Tarawas; Tondeos bolaj; Tána'a; Waelisi; Wawahoeling; Woea kopé motaha

Sulawesi distribution - North, Central, South, South-East

Altitude $-0-3000 \mathrm{~m}$

Collection(s) - Alston 16033, 16170, 16198, 16270; Amir 195; bb 1835, 1842, 1843, 1853 , $1866,1867,1878,1881,1884,1891,1895$, $1908,1909,1919,1935,4188,6035,8575$, $14331,14434,15027,16982,17176,17684$, $18024,18682,18801,19396,19447,19592$, 19604, 20753, 20865, 20904, 21387, 21733 , 21734, 22989, 23896, 24076, 24088, 24090, 24097, 24501, 24992, 26018, 26290, 26297, $28220,28227,28229,28274,28292,28293$, 29016, 29193, 29465, 31846, 31892, 32513, 32525, 32595, 33081, 33120; Bloembergen 3940; Bünnemeyer 10894, 12122; Burley, Partomihardjo \& Rachman 4031; Cel I 37, II 203, II 239, II 245, II 247, II 268, II 269 , II 270 , II 271 , II 272, II 293, II 311 , II 320 , II 327 , II 341 , II 350 , II 385 , II 410, II 419 , II 436, II 456a, II 469, II 497, III 31, III 48, III 126, III 486, IV 80, IV 120, V 170, V 189, V 218, V 220, V 221; Coode 5974, 5982 , 6037, 6108; Darnaedi 2195; De Vogel 2447, $2527,2532,2590,2651,5086,5166,5223$, 5274, 5293, 5398, 5405, 5413, 5414, 5416, $5462,5465,5497,5599,5660,5683,5699$, $5713,5751,5758,5810,5837,5865,5866$, 5968, 5980, 5981, 6073, 6087, 6094, 6134, $6138,6141,6235,6238,6268,6270,6271$, $6272,6277,6320,6340,6357,6368,6383$; De Vogel \& Vermeulen 6602, 6632, 6728, $6838,6892,6910,7085,7145$; Elbert 3149 , $3342,3354,3374,3418,3476$; Eyma 520 , $525,596,1310,1352,1649,3351,3484$, $3624,3637,3646$; Forman $267,311,322$, 359; Hennipman 5675, 5804; Hose 802; Keßler et al. PK 3127; Koorders 18234 , $18235,18236,18237,18238,18241,18242$, 
$18243,18248,18250,18251,18252,18253$, $18254,18255,18256,18257,18258,18259$, $18260,18261,18262,18263,18264,18265$, $18266,18267,18269,18271,18272,18273$, $18276,18277,18278,18279,18280,18281$, $18282,18284,18285,18286,18287,18288$, $18289,18290,18291,18292,18293,18294$, $18295,18296,18297,18298,18300,18301$, $18302,18303,18304,18314,18315,18316$, $18317,18318,18323,24074$; Lam 2623, 2665, 2886, 2940, 2986, 3098, 3442; McDonald \& Ismail Cat 3859, Cat 4014, Cat 4054; Meijer 9572, 10125, 10719, 11069 , $11070,11165,11285,11292$; Noerkas 324 , 417; Prawiroatmodjo \& Maskuri 1231, 1494; Prawiroatmodjo \& Soewoko 1692, 1725 , $1744,1753,1776,1807,1813,1892,1920$, 1942, 1955, 1957, 1966, 1971; Rachmat 77, 737, 919; Ramlanto 145, 211; Ramlanto \& Fanani 608, 615; Tantra 1545, 1585, 1589., 1592, 1596; Teijsmann HB 14007; Van Balgooy $3003,3237,3295,3297,3371,3423$, $3638,3647,3687,3717,3766,3767,3806$, $3838,3850,3855,3865,3879,3890,3893$, 3956, 3976, 4020, 4021, 4061, 4065, 4085 $A+B, 4088$; Van Steenis 10312; Whitmore \& Sidiyasa TCW 3519

\section{TRISTANIOPSIS}

\section{Tristaniopsis spec.}

Sulawesi distribution - South

Collection(s) - Kofman 124

\section{XANTHOMYRTUS}

\section{Xanthomyrtus angustifolius A.J. Scott}

Sulawesi distribution - Central

Lore Lindu National Park \& environs - present Altitude - $2450 \mathrm{~m}$

Collection(s) - Eyma 3958; Van Balgooy 3322

Xanthomyrtus flavida (Stapf) Diels

Sulawesi distribution - Central

Lore Lindu National Park \& environs — present Altitude - 1700-1800 m

Collection(s) - Eyma 1632a

\section{XANTHOSTEMON}

\section{Xanthostemon confertiflorum Merr.}

Sulawesi distribution - North, Central, South, South-East
Altitude - 5-900 m

Collection(s) - bb 18010, 19636, 21637, 21782, 22723, 22726, 31512; Cel 103; Elbert 3458, 3481; Kofman 104, 110; Ramlanto 160; Van Balgooy 3650, 3700, 3801

\section{Xanthostemon spec.}

Vernacular name(s) - Koloju perempuan [Soroako]

Sulawesi distribution - South

Altitude - 300-420 m

Collection(s) - De Vogel 6090, 6228, 6345; Meijer 11103, 11180

\section{NYCTAGINACEAE}

\section{BOERHAAVIA}

\section{Boerhaavia diffusa L.}

Sulawesi distribution - North, Central, South Lore Lindu National Park \& environs - present Altitude $-0-100 \mathrm{~m}$

Collection(s) - Eyma 1737, 1769; Hennipman 5012; Kaudern 529; Meijer 9216; Noerkas 22, 170, 276; Teijsmann HB 13899

\section{Boerhaavia spec.}

Sulawesi distribution - Central Lore Lindu National Park \& environs - present Collection(s) - Meijer 9175

\section{PISONIA}

\section{Pisonia aculeata $\mathrm{L}$.}

Sulawesi distribution - South, South-East Altitude $-0-320 \mathrm{~m}$

Collection(s) - Elbert 2590, 2757; Teijsmann HB 12272, 13830; Zollinger 3320

Pisonia cauliflora Scheff.

Vernacular name(s) - Nozing'a

Sulawesi distribution - North

Altitude - 20-60 m

Collection(s) - Lam 2537, 3004

Pisonia umbellifera (G. Forst.) Seem.

Vernacular name(s) - Androeling; Saoenkapaja; Palindo; Tawura [Tolaki]

Sulawesi distribution - North, Central, South, South-East 
Altitude - 4-750 m

Collection(s) - bb 6031, 21322, 21379; Bünnemeyer 11523; Burley, Partomihardjo \& Rachman 4032; Cel I 75, V 181; Coode 6063; Eyma 3810; Meijer 10943; Noerkas 263; Teijsmann HB 12370, HB 12633, HB 12767

\section{OCHNACEAE}

\section{BRACKENRIDGEA}

Brackenridgea palustris Bartell. subsp. kjellbergii Kanis

Sulawesi distribution - South Altitude - $400 \mathrm{~m}$

Collection(s) - Kjellberg 2046, 2808

\section{GOMPHIA}

\section{Gomphia serrata (Gaertn.) Kanis}

Vernacular name(s) - Lebani; Morosisio [Palu] Sulawesi distribution - South

Altitude - 10-400 m

Collection(s) - bb 2386, 2413, 13584, 24099; Cel II 438, III 34, IV 194; Kjellberg 1172

\section{SCHUURMANSIA}

Schuurmansia elegans Blume

Sulawesi distribution - Central

Altitude - $1050 \mathrm{~m}$

Collection(s) - bb 24127

\section{OLACACEAE}

\section{ANACOLOSA}

\section{Anacolosa frutescens Blume}

Sulawesi distribution - North

Altitude - 0-1100 m

Collection(s) - bb 17293, 29014; Koorders 17477,18366

\section{ERYTHROPALUM}

Erythropalum scandens Blume

Sulawesi distribution - North

Altitude - 50-700 m

Collection(s) - Forman 308; Lam 2806
OLAX

Olax imbricata Roxb.

Sulawesi distribution - South, South-East

Altitude - 0-400 m

Collection(s) - De Vogel 5776; Elbert 2656, $2742,2771,3314$

\section{STROMBOSIA}

\section{Strombosia spec.}

Sulawesi distribution - South

Altitude - 300 m

Collection(s) - Chin 3419; De Vogel 6356

\section{XIMENIA}

\section{Ximenia americana $L$.}

Vernacular name(s) - Lemo-lemo

Sulawesi distribution - North, Central

Altitude - 0-1 m

Collection(s) - Lam 2508; Rachmat 341

\section{OLEACEAE}

\section{CHIONANTHUS}

Chionanthus cordulatus Koord.

Sulawesi distribution - North

Altitude $-500-700 \mathrm{~m}$

Collection(s) - Koorders 19569, 19592

Chionanthus laxiflorus Blume

Vernacular name(s) - kolaka motaha

Sulawesi distribution - Central, South

Lore Lindu National Park \& environs — present

Altitude - 50-2000 m

Collection(s) - bb 1846; Cel III 20, III 71; De Vogel 3477, 6097; Johansson 329; Meijer 9473A; Tantra 1580, 1582; Van Balgooy 3190, 3396, 3401, 3859; Van Vuuren 709

Chionanthus montanus Blume

Sulawesi distribution - North, Central

Lore Lindu National Park \& environs - present Altitude - 60-500 m

Collection(s) - bb 18803; Burley, Partomihardjo \& Rachman 3820 
Chionanthus nitens Koord. \& Valeton

Sulawesi distribution - North

Altitude - 350-750 m

Collection(s) - Burley, Partomihardjo \& Rach$\operatorname{man} 3819,3992$

Chionanthus pluriflorus (Knobl.) Kiew

Sulawesi distribution - Central

Altitude $-200 \mathrm{~m}$

Collection(s) — Ramlanto \& Fanani 609

Chionanthus ramiflorus Roxb.

Vernacular name(s) - Bintunu [Besoa]

Sulawesi distribution - North, Central, South

Lore Lindu National Park \& environs - present

Altitude - 20-1050 m

Collection(s) - bb 22951; Coode 6278; De Vogel 5602, 5922, 6297; Forsten 389; Hennipman 5761; Koorders 18372, 18383; McDonald \& Ismail 3889; Meijer 10181; Teijsmann HB 11865, HB 12128; Van Balgooy 3681; Van Vuuren 380; Widjaja 752

Chionanthus rupicolus (Lingelsh.) Kiew

Sulawesi distribution - South, South-East

Altitude - 300-900 m

Collection(s) - De Vogel 5835, 6325, 6328, 6347; Elbert 3470, 3484; Van Balgooy 3791

Chionanthus sordidus Kiew

Vernacular name(s) - Parasingo poeti

Sulawesi distribution - South

Altitude $-100-430 \mathrm{~m}$

Collection(s) - Cel II 260, II 310; De Vogel 6244; Meijer 11436; Ramlanto 203; Teijsmann HB 13941

Chionanthus stenurus (Merr) Kiew

Sulawesi distribution - South

Altitude $-50-400 \mathrm{~m}$

Collection(s) —De Vogel 5792, 5892; Kjellberg 2120

Chionanthus sulawesicus Kiew

Vernacular name(s) - Kuhe [Besoa]; Loya

Sulawesi distribution - Central

Lore Lindu National Park \& environs - present

Altitude - 200-2200 m

Collection(s) - Cel V 256; Darnaedi 1623; De Vogel 5096, 5208, 5534, 5535, 5568; Johans- son 552; Meijer 9563; Van Balgooy 3046, 3068

\section{Chionanthus spec.}

Sulawesi distribution - North

Altitude - 300-1500 m

Collection(s) - Burley, Partomihardjo \& Rachman 3938; De Vogel \& Vermeulen 6951; Milliken 995

\section{JASMINUM}

Jasminum aemulum R. Br.

Vernacular name(s) - Moenoertongke

Sulawesi distribution - North, Central, South, South-East

Altitude - 20-1700 m

Collection(s) - Darnaedi 1974; Elbert 2728; Eyma 1181, 3503; Forsten 356, 362; Prawiroatmodjo \& Soewoko 1993; Van Balgooy 2970,3607

\section{Jasminum carinatum Blume}

Sulawesi distribution - North

Altitude - $220 \mathrm{~m}$

Collection(s) - De Vogel \& Vermeulen 6462, 6683; Weir 10

Jasminum crassifolium Blume

Sulawesi distribution - North

Altitude - 350-600 m

Collection(s) - De Vogel \& Vermeulen 7006; De Vriese \& Teijsmann 90; Kaudern 63

Jasminum didymum G. Forst.

Sulawesi distribution - North, Central

Lore Lindu National Park \& environs - present Altitude - 0-200 m

Collection(s) - Koorders 18374; Van Balgooy 2993

Jasminum griffithii C.B. Clarke

Sulawesi distribution - South

Altitude $-400 \mathrm{~m}$

Collection(s) - Cel V 252

Jasminum multiflorum Roth

Sulawesi distribution - South

Altitude $-250 \mathrm{~m}$

Collection(s) - Ramlanto 240 
Jasminum papuasicum Lingelsh.

Sulawesi distribution - North

Altitude $-50 \mathrm{~m}$

Collection(s) - Lam 3086

Jasminum sambac (L.) Aiton

Sulawesi distribution - North, South-East

Collection(s) - Koorders 18373; Widjaja 642

Jasminum simplicifolium G. Forst.

Sulawesi distribution - North

Lore Lindu National Park \& environs'- present

Altitude $-30 \mathrm{~m}$

Collection(s) — De Vogel 5019

Jasminum spec.

Sulawesi distribution - North, Central, SouthEast

Altitude - 600-1500 m

Collection(s) - Kaudern 63; Keßler et al. PK 3143; McDonald \& Ismail 4069

\section{LIGUSTRUM}

\section{Ligustrum glomeratum Blume}

Vernacular name(s) - Boenesaka

Sulawesi distribution - South

Altitude - 400-1900 m

Collection(s) - bb 8570, 20425; Chin 3673;

Kaudern 517; Van Vuuren 980

\section{MYXOPYRUM}

Myxopyrum nervosum Blume

Sulawesi distribution - North, South

Altitude $-300 \mathrm{~m}$

Collection(s) — De Vogel 6389; Koorders 18377

\section{Myxopyrum ovatum Hill}

Sulawesi distribution - North

Altitude - $100 \mathrm{~m}$

Collection(s) - Lam 2619

\section{OPILIACEAE}

\section{CHAMPEREIA}

Champereia manillana (Blume) Merr.

Vernacular name(s) - amaloan'a; aramalu
Sulawesi distribution - North, South, SouthEast

Altitude $-0-300 \mathrm{~m}$

Collection(s) - Elbert 2968, 2989, 3280; Kjellberg 2159; Lam 3162, 3419; Noerkas 376; Teijsmann HB 12298, HB 13844

\section{OPILIA}

Opilia amentacea Roxb.

Sulawesi distribution - South, South-East Altitude $-0-25 \mathrm{~m}$

Collection(s) - Elbert 2967; Kjellberg 2027; Soenarko 293

\section{OXALIDACEAE}

AVERRHOA

\section{Averrhoa bilimbi L.}

Sulawesi distribution - North, South-East

Altitude - 0-150 m

Collection(s) - Burley, Partomihardjo \& Rachman 4082; De Vogel 2524; Elbert 2596; Koorders 17164; Meijer 10057

\section{Averrhoa carambola L.}

Sulawesi distribution - North, Central

Altitude - 220-950 m

Collection(s) - Bünnemeyer 11218; De Vogel \& Vermeulen 6488

\section{BIOPHYTUM}

\section{Biophytum fruticosum Blume}

Sulawesi distribution - Central

Lore Lindu National Park \& environs - present Altitude $-200 \mathrm{~m}$

Collection(s) - Kaudern 438; Van Balgooy 2994

Biophytum sensitivum (L.) DC.

Sulawesi distribution - North, Central, South, South-East

Altitude - 0-150 m

Collection(s) - Elbert 2602, 3008; Forsten 431; Kaudern 117; Van Vuuren 105, 140 


\section{SARCOTHECA}

Sarcotheca celebica Veldk.

Sulawesi distribution - South

Collection(s) - Cel II 439

\section{PALMAE}

\section{CALAMUS}

Calamus didymocarpus Warb.

Sulawesi distribution - North, South

Altitude - 700-2060 m

Collection(s) - Bünnemeyer 12020; Dransfield \& Mogea JD 3857; Koorders 18392

Calamus koordersianus Becc.

Sulawesi distribution - North

Altitude $-100 \mathrm{~m}$

Collection(s) - Dransfield 3815; Koorders 18400

Calamus leptostachys Becc.

Sulawesi distribution - Central

Altitude $-200 \mathrm{~m}$

Collection(s) - Ramlanto \& Fanani 604

Calamus macrosphaerion Becc.

Sulawesi distribution - North, South, SouthEast

Altitude - 220-1200 m

Collection(s) - Darnaedi 2074; Mogea 1347, 5077

Calamus minahassae Warb.

Sulawesi distribution - North, Central

Altitude - 400-1000 m

Collection(s) - De Vogel 5209; Dransfield \& Mogea JD 3696, JD 3739, JD 3741; Johansson 110; Koorders 18414

Calamus ornatus Blume var. celebicus Becc.

Sulawesi distribution - North

Altitude - 200-220 m

Collection(s) — De Vogel \& Vermeulen 6748; Mogea 5041

Calamus orthostachyus Furtado

Sulawesi distribution - Central
Lore Lindu National Park \& environs - present

Altitude $-2000 \mathrm{~m}$

Collection(s) - Mogea 1329

Calamus pseudo-mollis Becc.

Sulawesi distribution - North

Altitude $-50 \mathrm{~m}$

Collection(s) - Koorders 18395, 18413

Calamus robinsonianus Becc.

Sulawesi distribution - South

Collection(s) - bb 17721

Calamus scleracanthus Becc. ex Heyne

Sulawesi distribution - North

Altitude $-40 \mathrm{~m}$

Collection(s) - Dransfield \& Mogea JD 3760

Calamus siphonospathus Mart. var. dransfieldii A. Baja-Lapis

Sulawesi distribution - North

Altitude - $500 \mathrm{~m}$

Collection(s) - Dransfield \& Mogea JD 3738

Calamus subidermis Wendl. ex Becc.

Sulawesi distribution - North

Altitude $-50 \mathrm{~m}$

Collection(s) - Dransfield \& Mogea JD 3720

Calamus subinermis Wendl. ex Becc.

Sulawesi distribution - North

Altitude $-50-70 \mathrm{~m}$

Collection(s) - Dransfield \& Mogea JD 3721, JD 3781, JD 3785

Calamus symphysipus Martelli

Sulawesi distribution - North, South

Altitude - 40-200 m

Collection(s) - Dransfield \& Mogea JD 3774, JD 3775; Koorders 18403, 18409, 18411, 18412; Meijer 10808; Mogea 5042

\section{Calamus spec.}

Vernacular name(s) - Lauro toulalai; Rotan Batu

Sulawesi distribution - North, Central, South

Altitude - 150-2000 m

Collection(s) - Burley, Partomihardjo \& Rachman 3630; De Vogel 5210, 5334, 5468, 5482, 
5943, 6124, 6182; Dransfield \& Mogea JD 3823, JD 3855, JD 3858, JD 3859, JD 3865; Johansson 113; Lam 2937, 3527; Meijer 9579, 10011, 10938; Mogea 1328

\section{CARYOTA}

Caryota mitis Lour.

Sulawesi distribution - North

Altitude $-50 \mathrm{~m}$

Collection(s) — Dransfield \& Mogea JD 3812

Caryota rumphiana Martelli

Sulawesi distribution - North

Altitude $-500 \mathrm{~m}$

Collection(s) - Koorders 18420

\section{CORYPHA}

\section{Corypha sylvestris Mart.}

Sulawesi distribution - South

Collection(s) - Van Vuuren 12

\section{DAEMONOROPS}

Daemonorops macroptera Becc.

Sulawesi distribution - North, Central

Altitude - 220-730 m

Collection(s) - Johansson 169; Mogea 5076

Daemonorops macropterus Miq.

Sulawesi distribution - North

Collection(s) - Riedel 84

Daemonorops riedelianus (Miq.) Becc.

Sulawesi distribution - North

Altitude $-50-500 \mathrm{~m}$

Collection(s) - Dransfield \& Mogea JD 3737, JD 3787; Koorders 18389, 18396; Riedel 85

Daemonorops robusta Warb.

Sulawesi distribution - North

Altitude - 50-700 m

Collection(s) - Dransfield \& Mogea 3800; Koorders 18140, 18399

\section{Daemonorops sarasinorum Warb.}

Sulawesi distribution - North Altitude $-50-1000 \mathrm{~m}$
Collection(s) — Dransfield \& Mogea JD 3744, JD 3862; Koorders 18388, 18391

\section{Daemonorops spec.}

Sulawesi distribution - South-East

Altitude $-0-250 \mathrm{~m}$

Collection(s) - Prawiroatmodjo \& Soewoko 1799

\section{GRONOPHYLLUM}

\section{Gronophyllum microspadix Burret}

Sulawesi distribution - South

Altitude - $400 \mathrm{~m}$

Collection(s) - Van Balgooy 3733

\section{KORTHALSIA}

\section{Korthalsia celebica Becc.}

Sulawesi distribution - North, South

Altitude - 40-430 m

Collection(s) - De Vogel 6249; De Vogel \& Vermeulen 7002; Dransfield \& Mogea JD 3773, JD 3837, JD 3838; Furtado 30812; Koorders 18398

\section{Korthalsia spec.}

Sulawesi distribution - North, South-East Altitude - 20-250 m

Collection(s) - Mogea 5093; Prawiroatmodjo \& Soewoko 2006

\section{LICUALA}

\section{Licuala celebica Miq.}

Sulawesi distribution - North, Central Lore Lindu National Park \& environs - present Altitude $-800 \mathrm{~m}$

Collection(s) - Meijer 9336, 10090

\section{Licuala spec.}

Sulawesi distribution - Central, South-East Lore Lindu National Park \& environs - present Altitude $-20-500 \mathrm{~m}$

Collection(s) - De Vogel 5677; Prawiroatmodjo \& Soewoko 1760 
LIVISTONA

Livistona rotundifolia (Lam.) Martelli

Sulawesi distribution - North

Altitude - 50-220 m

Collection(s) - De Vogel \& Vermeulen 6894;

Dransfield \& Mogea JD 3831

\section{ONCOSPERMA}

Oncosperma horridum Scheff.

Vernacular name(s) - Metejongan [Kotamobagu]

Sulawesi distribution - North

Altitude $-220 \mathrm{~m}$

Collection(s) - De Vogel \& Vermeulen 6813

\section{PHOLIDOCARPUS}

Pholidocarpus ihur Becc.

Sulawesi distribution - North

Altitude - $200 \mathrm{~m}$

Collection(s) - Mogea 5097

\section{PIGAFETTA}

\section{Pigafetta filaris Becc.}

Sulawesi distribution - North

Altitude - $900 \mathrm{~m}$

Collection(s) - Burck 1483; Dransfield \& Mogea JD 3867

\section{PINANGA}

\section{Pinanga caesia Blume}

Sulawesi distribution - North

Altitude $-1200 \mathrm{~m}$

Collection(s) - Forsten 4; Koorders 18432, 18435

Pinanga insignis Becc.

Sulawesi distribution - Central

Lore Lindu National Park \& environs - present

Altitude $-650 \mathrm{~m}$

Collection(s) - Johansson 165

\section{Pinanga spec.}

Vernacular name(s) - Saliho

Sulawesi distribution - North, Central, South, South-East
Altitude $-20-1000 \mathrm{~m}$

Collection(s) - De Vogel 5064; De Vogel \& Vermeulen 6694, 7110; Dransfield \& Mogea 3830, JD 3715, JD 3717, JD 3736; Johansson 126; Lam 2439; McDonald \& Ismail 3888; Meijer 9378; Prawiroatmodjo \& Soewoko 1748, 1974; Van Balgooy 3926

\section{PANDANACEAE}

\section{FREYCINETIA}

\section{Freycinetia amboinensis Martelli}

Sulawesi distribution - North

Altitude $-150 \mathrm{~m}$

Collection(s) - Lam 2891

Freycinetia celebica Solms

Vernacular name(s) - Aretes [Tombulu]

Sulawesi distribution - North

Altitude $-0-1100 \mathrm{~m}$

Collection(s) - Alston 16164; Forman 380; Forsten 13

\section{Freycinetia ceramensis Martelli}

Sulawesi distribution - North, South-East

Altitude - 20-400 m

Collection(s) - Koorders 18459; Prawiroatmodjo \& Soewoko 1889

Freycinetia devriesei Solms

Vernacular name(s) - Wuwuk

Sulawesi distribution - North

Collection(s) - Alston 16488

Freycinetia funicularis (Sav. ex Lam.) Merr.

Sulawesi distribution - South

Altitude - $700 \mathrm{~m}$

Collection(s) - Bünnemeyer 11421

Freycinetia insignis Blume subsp. hispidula B.C. Stone

Sulawesi distribution - South

Altitude - $350 \mathrm{~m}$

Collection(s) - Van Balgooy 4035

Freycinetia koordersiana Martelli

Sulawesi distribution - North

Collection(s) - Koorders 18461 
Freycinetia kostermansii Stone

Sulawesi distribution - South

Altitude $-400 \mathrm{~m}$

Collection(s) - Van Balgooy 3790

Freycinetia megacarpa Merr.

Sulawesi distribution - South

Altitude - $400 \mathrm{~m}$

Collection(s) - De Vogel 6084

Freycinetia micrura B.C. Stone

Sulawesi distribution - Central

Lore Lindu National Park \& environs - present

Altitude $-2000 \mathrm{~m}$

Collection(s) - Van Balgooy 3216

Freycinetia multiflora Merr.

Sulawesi distribution - Central

Lore Lindu National Park \& environs - present

Altitude - $2200 \mathrm{~m}$

Collection(s) - Bloembergen 3974

Freycinetia radicans Gaudich.

Sulawesi distribution - South

Altitude - $2060 \mathrm{~m}$

Collection(s) - Bünnemeyer 12016

Freycinetia scandens Gaudich.

Sulawesi distribution - Central, South-East

Altitude - 125-1000 m

Collection(s) - Elbert 3043; Meijer 9940; Van

Balgooy 3064, 3118

Freycinetia trachypoda Merr. \& Perry

Sulawesi distribution - South

Altitude $-400 \mathrm{~m}$

Collection(s) - Van Balgooy 4063

Freycinetia spec.

Sulawesi distribution - North, Central, South, South-East

Altitude - 125-2500 m

Collection(s) - Bloembergen 3945; De Vogel 2613, 5297, 6137; Elbert 3044, 3103, 3144; Hennipman 5579, 6078; Monod de Froideville 169; Yusuf \& Wahyono 81, 134

\section{PANDANUS}

\section{Pandanus brachyspathus Martelli}

Sulawesi distribution - Central, South

Lore Lindu National Park \& environs - present Altitude - $970 \mathrm{~m}$

Collection(s) - Meijer 9146, 9939

Pandanus dipsaceus Martelli

Sulawesi distribution - South-East

Altitude - 20-250 m

Collection(s) - Prawiroatmodjo \& Soewoko 1976

Pandanus dubius Spreng.

Vernacular name(s) - ababa'a

Sulawesi distribution - North

Altitude $-2 \mathrm{~m}$

Collection(s) — Lam 3041

Pandanus ellipsoideus Warb.

Vernacular name(s) - Lewayo(Napu)Naho nét [Uma]

Sulawesi distribution - Central

Altitude - $1200 \mathrm{~m}$

Collection(s) - Van Balgooy 3147

Pandanus polycephalus Lam.

Vernacular name(s) - Silar

Sulawesi distribution - North

Altitude $-300 \mathrm{~m}$

Collection(s) — De Vogel 2464

Pandanus sarasinorum Warb.

Sulawesi distribution - North, Central

Altitude $-2000 \mathrm{~m}$

Collection(s) - Forman 383; Milliken 1090; Van Balgooy 3251

Pandanus sulawesicus B.C. Stone

Sulawesi distribution - Central

Altitude - $1000 \mathrm{~m}$

Collection(s) - Van Balgooy 3107

Pandanus tectorius Parkinson

Vernacular name(s) - Pandan'a

Sulawesi distribution - North

Altitude $-350 \mathrm{~m}$

Collection(s) - Lam 3285 


\section{Pandanus spec.}

Sulawesi distribution - North, Central, South, South-East

Altitude - 20-1100 m

Collection(s) - De Vogel 5264, 5287, 6044, 6146; De Vogel \& Vermeulen 6826; Hennipman 6115; Meijer 11212a; Prawiroatmodjo \& Soewoko 1818

\section{PITTOSPORACEAE}

\section{PITTOSPORUM}

\section{Pittosporum ferrugineum Aiton}

Vernacular name(s) - Milai; Riboesara; Ririk; Safe

Sulawesi distribution - North, South, SouthEast

Altitude $-20-1200 \mathrm{~m}$

Collection(s) - bb 9, 4183, 5454, 5847; Elbert 3460; Lam 2663; Teijsmann HB 12237

Pittosporum moluccanum (Lam.) Miq.

Vernacular name(s) - Bata bata borong

Sulawesi distribution - North, Central, South

Lore Lindu National Park \& environs - present

Altitude - 0-2350 m

Collection(s) - Alston 16100; Cel I 56; De Vogel 5342, 5346, 5362, 5418, 5421; Elbert 3249, 3497; Meijer 9144; Teijsmann HB 13949; Van Balgooy 3177; Yoshida 1177

Pittosporum pentandrum (Blanco) Merr.

Sulawesi distribution - North, Central

Lore Lindu National Park \& environs — present

Altitude - 150-900 m

Collection(s) - Meijer 10217; Van Balgooy 2971

Pittosporum ramiflorum (Zoll. \& Moritzi) Zoll. ex Miq.

Vernacular name(s) - Mawoering

Sulawesi distribution - North, Central, South

Altitude - 0-2200 m

Collection(s) - Alston 16074; bb 13516; De Vogel 2432, 2624; Hennipman 5807; Koorders 18511; Tantra 1570; Teijsmann HB 13750

\section{Pittosporum spec.}

Sulawesi distribution - South

Collection(s) - Chin 3509, 3549

\section{PLUMBAGINACEAE}

\author{
PLUMBAGO
}

Plumbago indica L.

Sulawesi distribution - South

Altitude $-950 \mathrm{~m}$

Collection(s) - Bünnemeyer 11344

\section{PODOCARPACEAE}

\section{DACRYCARPUS}

Dacrycarpus cinctus (Pilg.) de Laub.

Sulawesi distribution - Central, South

Lore Lindu National Park \& environs - present

Altitude - 900-2250 m

Collection(s) - bb 13633, 24958; Bloembergen 3976, 3977; Meijer 9877; Tantra 1572; Van Balgooy 3248

Dacrycarpus imbricatus (Blume) de Laub.

Vernacular name(s) - Kajoe Parang

Sulawesi distribution - South

Altitude - 1550-2860 m

Collection(s) - bb 5460, 20202, 29195; Bünnemeyer 11903, 12019; Eyma 570, 1369; Meijer 11038; Monod de Froideville 173

Dacrycarpus imbricatus (Blume) de Laub. var. patulus de Laub.

Vernacular name(s) - siori

Sulawesi distribution - Central, South

Altitude - 1200-2500 m

Collection(s) - bb 15155, 19599, 24951, 24956, 24957

Dacrycarpus steupii (Wasscher) de Laub.

Sulawesi distribution - Central, South

Altitude - 2350-3000 m

Collection(s) - bb 22857; Eyma 572, 675, 778; Van Balgooy 3333

\section{Dacrycarpus spec.}

Sulawesi distribution - Central

Lore Lindu National Park \& environs - present Altitude $-1850 \mathrm{~m}$

Collection(s) - De Vogel 5151 


\section{DACRYDIUM}

Dacrydium novoguineense Gibbs

Sulawesi distribution - South, South-East

Altitude - 250-1900 m

Collection(s) — bb 20786, 26288; Elbert 3126

Dacrydium xanthandrum Pilg.

Sulawesi distribution - North, South

Altitude $-1700 \mathrm{~m}$

Collection(s) - bb 23045; Eyma 3873

Dacrydium spec.

Vernacular name(s) - Tute

Sulawesi distribution - Central, South, SouthEast

Altitude - 380-1850 m

Collection(s) - Coode 6154; De Vogel 5140, $5864 \mathrm{~A}, 6335$

\section{DECUSSOCARPUS}

Decussocarpus wallichianus (Presl) de Laub.

Vernacular name(s) — Moosambonge [Tombolilato]; Tandanggoeli

Sulawesi distribution - North, Central, South

Altitude - 10-700 m

Collection(s) - bb 9696, 15602, 24489, 25541, 31500, 31506; Cel III 80, III 143; Van Balgooy 3844,3861

\section{Decussocarpus spec.}

Sulawesi distribution - South

Altitude $-420 \mathrm{~m}$

Collection(s) — De Vogel 6098

\section{FALCATIFOLIUM}

Falcatifolium gruezoi de Laub.

Sulawesi distribution - North, Central

Altitude - 1200-1400 m

Collection(s) - bb 17544, 21294; Eyma 3671

\section{PHYLLOCLADUS}

\section{Phyllocladus hypophyllus Hook.f.}

Vernacular name(s) - Empire; Mantjoejoe

Sulawesi distribution - Central, South

Lore Lindu National Park \& environs - present Altitude $-1100-2500 \mathrm{~m}$
Collection(s) - bb 15026, 15154, 20270, 20782, 24089; Eyma 1001, 3621; Sands 280; Tantra 1602; Van Balgooy 3327

\section{PODOCARPUS}

Podocarpus indonesiensis de Laub. \& Silba

Sulawesi distribution - South

Altitude - 2550-3000 m

Collection(s) - Eyma 982, 1034, 1406

Podocarpus levis de Laub.

Vernacular name(s) - Sanroe

Sulawesi distribution - North, South, SouthEast

Altitude - 200-1500 m

Collection(s) - bb 15085; Cel II 286, II 325; Elbert 3129; Koorders 16533; Van Balgooy 3804

Podocarpus neriifolius D. Don

Sulawesi distribution - North, Central, South

Altitude - 450-1700 m

Collection(s) - Koorders 16535, 16537, 16538; Meijer 9541, 11188; Van Balgooy 3407

\section{Podocarpus pilgeri Foxw.}

Sulawesi distribution -Central, South

Lore Lindu National Park \& environs - present Altitude - 2000-2700 m

Collection(s) - bb 20437; Monod de Froideville 175; Van Balgooy 3312

Podocarpus rubens de Laub.

Sulawesi distribution - Central, South

Altitude - 2500-3695 m

Collection(s) - Eyma 624, 989; Teijsmann HB 14121, HB 14191

\section{Podocarpus rumphii Blume}

Vernacular name(s) - Sandoe

Sulawesi distribution - North, South

Altitude - 600-900 m

Collection(s) - bb 9705; Koorders 16534; Teijsmann HB 14068

Podocarpus spec.

Vernacular name(s) - Karunia

Sulawesi distribution - Central, South

Altitude $-300-2450 \mathrm{~m}$ 
Collection(s) - De Vogel 5277, 5373, 6118, Helicia kjellbergii Sleumer var. calva Sleumer 6343; Keßler et al. PK 3157

\section{PRUMNOPITYS}

Prumnopitys amara (Blume) de Laub.

Sulawesi distribution - South

Altitude - 980-1600 m

Collection(s) - bb 20785, 28316; Teijsmann HB 14069

\section{POLYGALACEAE}

\section{XANTHOPHYLLUM}

\section{Xanthophyllum celebicum Meijden}

Sulawesi distribution - South

Altitude $-300 \mathrm{~m}$

Collection(s) - Cel 393

\section{Xanthophyllum tenuipetalum Meijden}

Sulawesi distribution - North, Central, South, South-East

Altitude - 5-1000 m

Collection(s) - bb 5868; Cel IV 126; Koorders

- 18523; Prawiroatmodjo \& Soewoko 1673; Tantra 1562

\section{PROTEACEAE}

\section{GREVILLEA}

\section{Grevillea elbertii Sleumer}

Sulawesi distribution - South-East

Altitude $-600-700 \mathrm{~m}$

Collection(s) - Elbert 3475

\section{HELICIA}

Helicia attenuata (Jack) Blume

Sulawesi distribution - South

Collection(s) - Noerkas 438

Helicia celebica Sleumer

Sulawesi distribution - North, South

Altitude - 450-1600 m

Collection(s) - Koorders 17516, 18513, 18529, 19582; Van Balgooy 3871
Sulawesi distribution - South

Altitude $-200 \mathrm{~m}$

Collection(s) - bb 20047

Helicia teysmanniana Sleumer

Sulawesi distribution - South

Altitude - 1700-2600 m

Collection(s) - bb 20428; Bünnemeyer 11934, 12320; Teijsmann HB 14117

\section{Helicia spec.}

Sulawesi distribution - South-East

Altitude - 200-300 m

Collection(s) - Prawiroatmodjo \& Soewoko 1929

\section{MACADAMIA}

\section{Macadamia hildebrandii Steenis}

Sulawesi distribution — South, South-East

Altitude $-60-1600 \mathrm{~m}$

Collection(s) - Amir 40; Coode 6198; De Vogel $5854,5965,6148,6206,6332,6341,6350$; Ramlanto 155, 210

\section{RANUNCULACEAE}

\section{CLEMATIS}

Clematis mollissima (Hallier f.) Eichler

Sulawesi distribution - South

Collection(s) - Teijsmann HB 13960

Clematis stenanthera Eichler

Sulawesi distribution - North

Collection(s) - Koorders 16385, 16526

Clematis tenuimarginata Eichler

Sulawesi distribution - South

Collection(s) - Eyma 4163

\section{RHAMNACEAE}

\section{ALPHITONIA}

Alphitonia excelsa (Fenzl) Reissek ex Endl. Vernacular name(s) - Kole 
Sulawesi distribution - South, South-East

Altitude - 50-850 m

Collection(s) - bb 22079; Cel II 475; De Vogel 6130; Elbert 11140, 11503; Hennipman 5975; Kjellberg 673; Noerkas 451; Van Balgooy 3891; Yusuf \& Wahyono 157

\section{Alphitonia zizyphoides A. Gray}

Vernacular name(s) - Tole

Sulawesi distribution - North

Altitude $-120-400 \mathrm{~m}$

Collection(s) - Lam 2651, 3102, 3276

\section{COLUBRINA}

\section{Colubrina asiatica Brongn.}

Sulawesi distribution - North, South, SouthEast

Altitude - 0-1000 m

Collection(s) - Alston 15994; Chin 3622; Elbert 3327; Forsten 441; Kaudern 442; Kjellberg 32

\section{EMMENOSPERMA}

Emmenosperma steenisiana M.C. Johnst.

Sulawesi distribution - South

Altitude $-300 \mathrm{~m}$

Collection(s) - Cel II 144, II 322; De Vogel 6396; Van Balgooy 3828

\section{GOUANIA}

\section{Gouania javanica Miq.}

Sulawesi distribution - North, Central

Altitude $-500 \mathrm{~m}$

Collection(s) - Burley, Partomihardjo \& Rachman 3622; Meijer 10233

\section{Gouania spec.}

Sulawesi distribution - North, Central, South Lore Lindu National Park \& environs - present Altitude $-50-1000 \mathrm{~m}$

Collection(s) - De Vogel 5184, 5557; Koorders 16943; Rachmat 90; Whitmore \& Sidiyasa TCW 3467

\section{VENTILAGO}

\section{Ventilago madraspatensis Gaertn.}

Sulawesi distribution - South-East

Altitude - 20-250 m

Collection(s) - Prawiroatmodjo \& Soewoko 1769

\section{Ventilago oblongifolia Blume}

Sulawesi distribution - North

Altitude $-75 \mathrm{~m}$

Collection(s) — De Vogel 2594

Ventilago spec.

Sulawesi distribution - South, South-East

Altitude $-0-400 \mathrm{~m}$

Collection(s) - De Vogel 5918; Elbert 2707, 3275; Hennipman 5818

\section{ZIZYPHUS}

Zizyphus angustifolius (Miq.) Hatus.

Sulawesi distribution - North, Central, South Lore Lindu National Park \& environs - present Altitude - 50-1000 m

Collection(s) - bb 17494, 23914; De Vogel \& Vermeulen 6607; Koorders 19071; Meijer 10033; Whitmore \& Sidiyasa TCW 3390

\section{Zizyphus oenoplia (L.) Mill.}

Sulawesi distribution - Central

Lore Lindu National Park \& environs - present Altitude - $400 \mathrm{~m}$

Collection(s) - Meijer 10168

\section{Zizyphus timorensis DC.}

Sulawesi distribution - North, South, SouthEast

Altitude - 2-300 m

Collection(s) - bb 16989, 19397, 21707, 24103, 25564; Cel I 18; Koorders 18543

\section{Zizyphus spec.}

Sulawesi distribution - Central, South-East Altitude - 20-1050 m

Collection(s) - De Vogel 5583; Prawiroatmodjo \& Soewoko 1727 


\section{RHIZOPHORACEAE}

\section{BRUGUIERA}

\section{Bruguiera cylindrica (L.) Blume}

Vernacular name(s) - Nanasi; Tangere

Sulawesi distribution - North, South, SouthEast

Altitude $-0-10 \mathrm{~m}$

Collection(s) - bb 3026, 22068; Elbert 3242. Lam 3401

\section{Bruguiera gymnorhiza (L.) Lam.}

Vernacular name(s) - Bangko; Moentoe; Nanasi wawine; Tjokke; Tongge; Tongkeh; Tonke

Sulawesi distribution - North, South, SouthEast

Altitude - 0-10 m

Collection(s) - Alston 16038; bb 3025, 3893, 13492, 14443, 15566, 22071, 32326; Béguin 1586; De Vriese 7; Elbert 3256; Forsten 25; Koorders 18531, 22229, 22290; Lam 2503, 2673; Meijer 9291; Rachmat 634; Teijsmann HB 13817

\section{Bruguiera parviflora Wight \& Arn.}

Vernacular name(s) - Adin Kadin; Poto-Poto; Sala; Tangke; Tideloeo

Sulawesi distribution - North, South, SouthEast

Altitude - 0-10 m

Collection(s) - bb 3892, 14439, 15571, 17060 , 19697, 22072; Forsten 15, 311; Kjellberg 1341; Rachmat 633, 787

\section{Bruguiera spec.}

Sulawesi distribution - North

Collection(s) - Koorders 18530, 18533

\section{CARALLIA}

Carallia brachiata (Lour.) Merr.

Vernacular name(s) - Bariraga; Morohoeko motea

Sulawesi distribution - North, Central, South, South-East

Altitude $-0-750 \mathrm{~m}$

Collection(s) - bb 5867, 21365, 24490; Burley, Partomihardjo \& Rachman 3817, 3860; Cel III 17; De Vogel 6106; Meijer 10051; Ramlanto \& Fanani 600; Teijsmann HB 11720

\section{CERIOPS}

Ceriops decandra (Griff.) Ding Hou

Vernacular name(s) - Tangere

Sulawesi distribution - North

Collection(s) - bb 15569

Ceriops tagal (Perry) C.B. Rob.

Vernacular name(s) - Kajoe Ting; Posi-Posi [Tonsea]; Tangalatoetoe; Tangir

Sulawesi distribution - North, South-East

Altitude - $1 \mathrm{~m}$

Collection(s) - Alston 16010; bb 3895, 14449 , 16712, 22070; De Vriese 611; Kjellberg 73; Meijer 9288

\section{GYNOTROCHES}

Gynotroches axillaris Blume

Sulawesi distribution - Central, South

Lore Lindu National Park \& environs - present

Altitude - 150-1700 m

Collection(s) - bb 20048, 24161, 24192; Van Balgooy 3389

\section{PELLACALYX}

Pellacalyx pustulatus Merr.

Sulawesi distribution - North

Altitude $-500 \mathrm{~m}$

Collection(s) — Koorders 18714, 18783

\section{RHIZOPHORA}

\section{Rhizophora apiculata Blume}

Vernacular name(s) - Bangko; Lenro; Oeroeroe; Polo

Sulawesi distribution - North, South, SouthEast

Altitude $-0-10 \mathrm{~m}$

Collection(s) - bb 3024, 3887, 13493, 13494; Cel III 72, III 148; De Vriese \& Teijsmann 62; Elbert 3237; Koorders 17961, 18542

Rhizophora mucronata Lam.

Vernacular name(s) - Opejo; Tongke djamin Sulawesi distribution - North, South, SouthEast

Altitude $-1 \mathrm{~m}$ 
Collection(s) - bb 3690, 3894, 16711; Lam 2674; Noerkas 56; Teijsmann HB 12110, HB 13816

\section{ROSACEAE}

\section{PRUNUS}

Prunus arborea (Blume) Kalkman var. alticola Kalkman

Sulawesi distribution - Central

Altitude - $2050 \mathrm{~m}$

Collection(s) - bb 26274

Prunus arborea (Blume) Kalkman var. arborea

Vernacular name(s) - Potosi

Sulawesi distribution - Central, South

Altitude - 25-800 m

Collection(s) - bb 22618, 24498, 28254; Cel II 379, IV 116; De Vogel 6207; Van Balgooy 3842

\section{Prunus clementis (Merr.) Kalkman}

Vernacular name(s) - Mororamba; Rampaloeria

Sulawesi distribution - North, South

Altitude - 230-900 m

Collection(s) - bb 17120; Burley, Partomihardjo \& Rachman 3754; Cel V 201; De Vriese \& Teijsmann 122; Koorders 18552, 18553, 18554, 18555, 18557; Meijer 11233

Prunus grisea (C. Müll.) Kalkman var. grisea

Vernacular name(s) - Boenobampo; Leka rintek; Ramba

Sulawesi distribution - North, Central, South, South-East

Altitude - 0-1300 m

Collection(s) - bb 9699; Cel I 67, II 233, II 358, II 364; De Vogel 2611, 2627, 5658, 5778, 5886, 5895, 6288; Meijer 9482; Prawiroatmodjo \& Soewoko 1826; Teijsmann HB 5852, HB 11741, HB 11791, HB 11984, HB 12206, HB 12216; Van Balgooy 4036, 4051; Whitmore \& Sidiyasa TCW 3522

Prunus grisea (Blume) Kalkman var. tomentosa (Koord. \& Valeton) Kalkman

Sulawesi distribution - South
Lore Lindu National Park \& environs - present Altitude - 1500-1700 m

Collection(s) - Meijer 9839

Prunus javanica (Teijsm. \& Binn.) Miq.

Vernacular name(s) - Boenobampoedja

Sulawesi distribution - North, South

Altitude $-200-600 \mathrm{~m}$

Collection(s) - Cel I 81; Koorders 18551; Teijsmann HB 13950

Prunus wallaceana Kalkman

Sulawesi distribution - North

Altitude - $50 \mathrm{~m}$

Collection(s) - Lam 2725; Teijsmann HB 12654

\section{Prunus spec.}

Sulawesi distribution - North, Central, South Altitude - 150-3300 m

Collection(s) - Burley, Partomihardjo \& Rachman 4063; Eyma 677; Van Balgooy 3328

\section{RUBIACEAE}

\section{ADINAUCLEA}

Adinauclea fagifolia (Teijsm. \& Binn. ex Havil.) Ridsdale

Vernacular name(s) - Hasi [Saloean]; Matambing lasi [Mahassan(?)al, Lowoe]; Motodoeto [Gorontalusch]

Sulawesi distribution - North, Central

Altitude - 10-150 m

Collection(s) - bb 5603, 26548, 27549, 31832, 31865,32367 ; Koorders 18635, 18636

\section{AIDIA}

\section{Aidia acuminata (Blume) K.M. Wong}

Sulawesi distribution - North, Central, South, South-East

Altitude $-550 \mathrm{~m}$

Collection(s) - Elbert 2580; Johansson 75; Koorders 18586, 18667, 18708; Lam 2429; Ramlanto \& Fanani 668; Van Balgooy 3923

Aidia bracteata Ridsdale

Sulawesi distribution - North, Central 
Altitude $-1500 \mathrm{~m}$

Collection(s) - De Vogel 2625, 5314; Forman 360

\section{Aidia heterophylla Ridsdale}

Sulawesi distribution - South

Altitude $-400 \mathrm{~m}$

Collection(s) - Van Balgooy 3645

Aidia racemosa (Cav.) Tirveng.

Sulawesi distribution - South

Collection(s) - Cel I 53

Aidia tomentosa (Blume) Ridsdale var. sundaica Ridsdale

Sulawesi distribution - Central

Collection(s) - De Vogel 5121, 5603

\section{AMARACARPUS}

Amaracarpus microphyllus Miq.

Sulawesi distribution - North

Collection(s) - Teijsmann HB 5352

Amaracarpus spec.

Sulawesi distribution - North

Altitude $-400 \mathrm{~m}$

Collection(s) - Afriastini 2894

\section{ANTHOCEPHALUS}

Anthocephalus chinensis (Lam.) Rich. ex Walp.

Sulawesi distribution - Central, South, SouthEast

Altitude $-70 \mathrm{~m}$

Collection(s) - bb 21600, 23308, 23309, 24707, 24998, 26285, 33092; De Vogel 5182

Anthocephalus macrophyllus (Roxb.) Havil.

Vernacular name(s) - Awá'a; Katoöe, Kokabo Sulawesi distribution - North, South, SouthEast

Altitude - 20-500 m

Collection(s) - bb 5865, 21598, 23093, 23606, 23940, 25543, 32355, 32372, 32613; Cel V 166, V 309; Forman 403; Koorders 18625, 18631, 18633; Lam 3030; Meijer 10055, 11402

\section{ANTIRHEA}

Antirhea hexasperma (Roxb.) Merr.

Sulawesi distribution - Central, South-East

Collection(s) - Coode 5888; Elbert 2609

Antirhea talaudensis Chaw

Sulawesi distribution - North

Altitude $-350 \mathrm{~m}$

Collection(s) - Lam 3260

BORRERIA

\section{Borreria spec.}

Sulawesi distribution - Central, South

Altitude - 50-650 m

Collection(s) - Buwalda 3750; De Vogel 6135; Johansson 264

\section{CANTHIUM}

\section{Canthium glabrum Blume}

Sulawesi distribution - North, South

Altitude - 200-500 m

Collection(s) - Burley, Partomihardjo \& Rachman 3603; Cel I 61a; Koorders 18580, 18581, 18583,18726

\section{Canthium monstrosum Merr.}

Vernacular name(s) - Gangáil'a

Sulawesi distribution - North

Altitude $-300 \mathrm{~m}$

Collection(s) — Lam 3294

\section{Canthium umbelligerum Miq.}

Sulawesi distribution - North

Altitude - $350 \mathrm{~m}$

Collection(s) - Lam 3310

Canthium spec.

Vernacular name(s) - Tondiot batu

Sulawesi distribution - North, South, SouthEast

Altitude - 20-1600 m

Collection(s) - bb 20214, 21716; Burley, Partomihardjo \& Rachman 3652; Cel II 480; De Vogel 5706, 6307, 6327, 6337, 6836; De Vogel \& Vermeulen 7059; Hennipman 5842; Milliken 1077; Ramlanto 205; Van Balgooy 3698; Zollinger 3326 


\section{CERISCOIDES}

Ceriscoides spec.

Sulawesi distribution - North

Collection(s) - Milliken 882

\section{CINCHONA}

\section{Cinchona succirubra Pav.}

Sulawesi distribution - South

Collection(s) - Afriastini 2127

\section{COFFEA}

\section{Coffea arabica L.}

Sulawesi distribution - South

Collection(s) - De Vriese \& Teijsmann 42; Rachmat 475

\section{DENTELLA}

Dentella repens (L.) J.R. Forst.

Sulawesi distribution - South

Collection(s) - Rant 892; Teijsmann HB 12091, HB 12808; Van Harreveld 8131

\section{DIPLOSPORA}

\section{Diplospora minahassae Koord.}

Sulawesi distribution - North

Altitude - 50-500 m

Collection(s) - De Vogel 2518; De Vogel \& Vermeulen 6973; Koorders 18589, 18590, 18737

\section{GAERTNERA}

\section{Gaertnera spec.}

Sulawesi distribution - North

Altitude $-1780 \mathrm{~m}$

Collection(s) - De Vogel \& Vermeulen 7191

\section{GARDENIA}

\section{Gardenia affinis Valeton}

Sulawesi distribution - North

Altitude - $1000 \mathrm{~m}$

Collection(s) - De Vogel 2616

\section{Gardenia forsteniana Miq.}

Sulawesi distribution - North, Central

Collection(s) - Burley, Partomihardjo \& Rachman 3681; Ramlanto \& Fanani 524

Gardenia pelenkahuana Teysm. \& Binn. ex Koord.

Sulawesi distribution - North

Collection(s) - De Vogel \& Vermeulen 6805; Kaudern 52; Koorders 24077

\section{Gardenia spec.}

Vernacular name(s) - Kawowo

Sulawesi distribution - North, South, SouthEast

Altitude $-10-1400 \mathrm{~m}$

Collection(s) - bb 6033, 20863; De Vogel 5757, 6211, 6317; Elbert 2990, 3454, 3490; Forman 323; Van Balgooy 3730, 3862

\section{GEOPHILA}

Geophila repens Lam.

Sulawesi distribution - North

Altitude $-220 \mathrm{~m}$

Collection(s) - De Vogel \& Vermeulen 6582

\section{GUETTARDA}

\section{Guettarda speciosa L.}

Sulawesi distribution - North, South

Collection(s) - Alston 16077; Hidayat AH 262;

Koorders 16280; Teijsmann HB 18035

\section{GYNOCHTHODES}

\section{Gynochthodes spec.}

Sulawesi distribution - North, Central, South Altitude - 100-500 m

Collection(s) - Darnaedi 1490; De Vogel 5747a, 5902, 6147, 6331; Hennipman 5841; Lam 3137, 3332; Van Balgooy 3846, 3944, 4038

\section{HEDYOTIS}

Hedyotis benguetensis Elmer

Sulawesi distribution - South

Collection(s) — Monod de Froideville 256 
Hedyotis biflora (L.) Lam.

Sulawesi distribution - Central

Collection(s) - Kaudern 378

Hedyotis brachypoda (DC.) Sivar. \& Biju

Sulawesi distribution - North, South

Altitude $-50 \mathrm{~m}$

Collection(s) - Buwalda 3732; Leemans 8136

Hedyotis buruensis (Miq.) Valeton ex Merr.

Sulawesi distribution - North

Altitude - $1780 \mathrm{~m}$

Collection(s) - De Vogel \& Vermeulen 7192

Hedyotis coryphoecetes Bremek.

Sulawesi distribution - South

Altitude $-2900 \mathrm{~m}$

Collection(s) - Zeef 230

Hedyotis costata (Roxb.) Kurz

Sulawesi distribution - South

Altitude $-1000 \mathrm{~m}$

Collection(s) - Bünnemeyer 11517

Hedyotis cyanantha Kurz

Sulawesi distribution - South

Altitude - 200-950 m

Collection(s) - Bünnemeyer 10996; Lam 3080

Hedyotis paniculata (L.) Lam.

Vernacular name(s) - Nenapu

Sulawesi distribution - North, Central

Altitude $-60-150 \mathrm{~m}$

Collection(s) - Kaudern 455; Lam 3082, 3399

Hedyotis pterita Blume

Sulawesi distribution - North, South-East

Altitude - 0-75 m

Collection(s) - Elbert 2887, 2915; Lam 3345; Noerkas 413

Hedyotis umbellata (L.) Lam.

Sulawesi distribution - North

Altitude $-2000 \mathrm{~m}$

Collection(s) - Koorders 18648

Hedyotis verticillata (L.) Lam.

Sulawesi distribution - South
Altitude $-20 \mathrm{~m}$

Collection(s) - Monod de Froideville 280

Hedyotis spec.

Sulawesi distribution - North, Central, South, South-East

Altitude - 10-3250 m

Collection(s) - Bloembergen 3985; Bünnemeyer 10649, 11009, 11202, 11247, 11257, 11704, 11884, 11928, 11998, 12171; De Vogel 5137, 5360; De Vogel \& Vermeulen 6815, 6821, 6881, 7041, 7057; Elbert 3170; Forman 399; Hennipman 5757; Johansson 208, 245, 280; Kofman 194, 248, 270; Koorders 18603, 18649, 18650, 18744; Meijer 9631, 9908; Milliken 1086; Prawiroatmodjo \& Maskuri 1437, 1442; Prawiroatmodjo \& Soewoko 1905, 1933; J.B.M. Smith 631; Van Balgooy 3159, 3874; Wardi Wd 002

\section{HYDNOPHYTUM}

\section{Hydnophytum formicarum Jack}

Sulawesi distribution — North, South-East

Altitude - 0-450 m

Collection(s) - Burley, Partomihardjo \& Rachman 4185; Elbert 2857; Forsten 12, 150; Koorders 18604, 18605; Lam 3283

\section{Hydnophytum spec.}

Sulawesi distribution - North, Central, South Altitude - 220-1000 m

Collection(s) — De Vogel 5938, 6374; De Vogel \& Vermeulen 6968; Hennipman 5663; Van Balgooy 3697,3835

\section{HYPOBATHRUM}

Hypobathrum microcarpum (Blume) Bakh.f. Sulawesi distribution - North

Altitude - 120

Collection(s) - De Vogel 2562

Hypobathrum spec.

Vernacular name(s) - Wombopale

Sulawesi distribution - North, Central, SouthEast

Altitude - 0-1500 m

Collection(s) - bb 15131, 22075; De Vogel 5309; De Vogel \& Vermeulen 6843; Elbert 
3233, 3264; Grimes 1155; Prawiroatmodjo \& Soewoko 1780

\section{IXORA}

\section{Ixora filipes Valeton}

Sulawesi distribution - North

Collection(s) - Koorders 18710

\section{Ixora grandifolia Zoll. \& Moritzi}

Sulawesi distribution - North

Altitude - $150 \mathrm{~m}$

Collection(s) - Burley, Partomihardjo \& Rachman 4212

\section{Ixora imitans Bremek.}

Sulawesi distribution - South-East

Collection(s) - Rachmat 690

Ixora makassarica Bremek.

Sulawesi distribution - South

Altitude $-200 \mathrm{~m}$

Collection(s) - Cel I 54

Ixora minahasae Bremek.

Sulawesi distribution - North

Altitude - $400 \mathrm{~m}$

Collection(s) - Koorders 18677

Ixora moluccana Bremek.

Sulawesi distribution - North

Altitude $-40 \mathrm{~m}$

Collection(s) - De Vogel 2578

Ixora philippinensis Merr.

Sulawesi distribution - Central

Collection(s) - Muat \& Grimes 1516

Ixora talaudensis Bremek.

Vernacular name(s) - Suwing'a

Sulawesi distribution - North

Altitude $-20-90 \mathrm{~m}$

Collection(s) - Lam 2547, 3185

\section{Ixora timorensis Decne.}

Sulawesi distribution - South, South-East

Altitude $-0-300 \mathrm{~m}$

Collection(s) - Elbert 3066, 3240, 3435; Teijsmann HB 12196, HB 14195
Ixora spec.

Sulawesi distribution - North, Central, South, South-East

Altitude - 0-2250 m

Collection(s) - Afriastini 1999, 2050; Alston 16099; Coode 5826, 5878, 6248; Darnaedi 1928; De Vogel 5817, 5901, 6133, 6269, 6334; De Vogel \& Vermeulen 6472, 6678, 7030,7072 ; Hennipman 5821, 6100; Johansson 326; Koorders 18689; Meijer 11440; Monod de Froideville 266; Prawiroatmodjo \& Maskuri 1230; Prawiroatmodjo \& Soewoko 1756, 1875, 1876, 1959; Ramlanto 139, 188, 192; Ramlanto \& Fanani 611, 749; Tantra 1573; Van Balgooy 3653, 3725, 3762; Whitmore \& Sidiyasa TCW 3461, TCW 3512

\section{KNOXIA}

Knoxia sumatrensis (Retz.) DC.

Sulawesi distribution - North, South, SouthEast

Altitude - 150-170 m

Collection(s) - Kjellberg 477; Lam 3436; Rachmat 218; Van Vuuren 373

\section{LASIANTHUS}

Lasianthus attenuatus Jack

Sulawesi distribution - South

Collection(s) - Teijsmann HB 13787

Lasianthus biflorus (Blume) Gangop. \& Chakrab.

Sulawesi distribution - Central

Lore Lindu National Park \& environs — present Altitude - $1700 \mathrm{~m}$

Collection(s) - Van Balgooy 3306

Lasianthus chlorocarpus K. Schum.

Sulawesi distribution - North, South

Altitude - 220-550 m

Collection(s) - De Vogel \& Vermeulen 6518, 6608; Hennipman 6118; Whitmore \& Sidiyasa TCW 3418

Lasianthus clementis Merr.

Sulawesi distribution - Central Lore Lindu National Park \& environs - present 


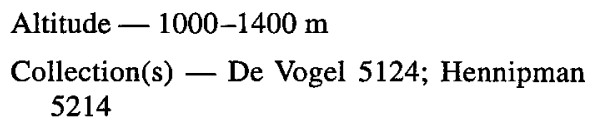

\section{Lasianthus fordii Hance}

Sulawesi distribution - North

Lore Lindu National Park \& environs — present

Altitude $-1600 \mathrm{~m}$

Collection(s) - Bloembergen 3927

Lasianthus hirtus Ridl.

Sulawesi distribution - South

Altitude $-850 \mathrm{~m}$

Collection(s) - Yusuf \& Wahyono 69

Lasianthus pedicellatus $\mathrm{H}$. Zhu

Sulawesi distribution - Central

Lore Lindu National Park \& environs - present

Altitude - 1850-1950 m

Collection(s) - De Vogel 5136, 5344

\section{Lasianthus reticulatus Blume}

Sulawesi distribution - South-East

Altitude $-100 \mathrm{~m}$

Collection(s) - Prawiroatmodjo \& Maskuri 1423

\section{Lasianthus rhinocerotis Blume}

Sulawesi distribution - North, South

Lore Lindu National Park \& environs - present Altitude - 850-1600 m

Collection(s) - Bloembergen 3939; Yusuf \& Wahyono 68

\section{Lasianthus stercorarius Blume}

Sulawesi distribution - North, Central

Altitude - $1420 \mathrm{~m}$

Collection(s) - Milliken 954; Van Balgooy 3009

\section{Lasianthus stipularis Blume}

Sulawesi distribution - Central

Lore Lindu National Park \& environs - present

Altitude $-650 \mathrm{~m}$

Collection(s) - Johansson 60

Lasianthus sylvestroides Valeton

Sulawesi distribution - North

Lore Lindu National Park \& environs - present Altitude $-1300-1600 \mathrm{~m}$
Collection(s) - Bloembergen 3906; Milliken
964

Lasianthus tomentosus Blume

Sulawesi distribution - South

Altitude $-950 \mathrm{~m}$

Collection(s) - Bünnemeyer 11010

\section{Lasianthus trichophlebus Hemsl.}

Sulawesi distribution - Central

Lore Lindu National Park \& environs - present

Altitude - 60-730 m

Collection(s) - Coode 5846; Johansson 118, 209

Lasianthus verticillatus (Lour.) Merr.

Sulawesi distribution - North

Altitude $-200 \mathrm{~m}$

Collection(s) - Church \& Ismail 10

\section{LUCINAEA}

Lucinaea spec.

Sulawesi distribution - North

Altitude $-1000 \mathrm{~m}$

Collection(s) - De Vogel \& Vermeulen 7175

\section{MASCHALOCORYMBUS}

Maschalocorymbus corymbosum (Blume) Bremek.

Sulawesi distribution - Central

Altitude $-300 \mathrm{~m}$

Collection(s) - Ramlanto \& Fanani 483

\section{MORINDA}

Morinda celebica Miq.

Sulawesi distribution - South

Collection(s) - Zollinger 3441

Morinda citrifolia L.

Vernacular name(s) - Bingkoeroe; seru

Sulawesi distribution - North, Central, South, South-East

Altitude - 0-950 m

Collection(s) - Bünnemeyer 10641, 11202 , 12484, 12577; De Vogel 6382; Elbert 2536; Hidayat AH 256; Lam 2442, 3357; Noerkas 
414; Rachmat 11, 144, 5803; Van Balgooy 3824; Van Vuuren 386

Morinda citrifolia L. var. bracteata (Roxb.) Hook.f.

Sulawesi distribution - North, South, SouthEast

Altitude - 0-600 m

Collection(s) - Afriastini 2013; Afriastini \& Rohajawati JJA 2973; De Vogel \& Vermeulen 6489; Elbert 2644, 3062, 3257; Kaudern 194; Meijer 10675; Ramlanto 243

Morinda tomentosa Roth.

Sulawesi distribution - South

Collection(s) - bb 8; Noerkas 412, 422

\section{Morinda spec.}

Vernacular name(s) - Sea [Tonsea]

Sulawesi distribution - North, Central, South, South-East

Altitude $-0-1100 \mathrm{~m}$

Collection(s) - Alston 16005, 16090; Buwalda 3695; Chin 3416; Coode 6120; De Vogel 6143, 6301; De Vogel \& Vermeulen 6584, 6674, 6675; Elbert 2802; Lam 3270; Prawiroatmodjo \& Soewokgo 1679, 1873; Van Balgooy 3886,4077 ; Whitmore \& Sidiyasa TCW 3379

\section{MUSSAENDA}

\section{Mussaenda frondosa L.}

Sulawesi distribution - Central, South

Altitude $-50 \mathrm{~m}$

Collection(s) - Afriastini 2095; Amir 42

\section{Mussaenda spec.}

Vernacular name(s) - Bali ade arena; Loenkang Sulawesi distribution - North, Central, South Altitude - 40-1000 m

Collection(s) - Alston 15904; Bloembergen 4062; Bünnemeyer 10637, 10772, 11036, 11739; Buwalda 3792; Coode 5977; De Vogel 5878; Johansson 533; Kaudern 421; Koorders 18616, 18617, 18618, 18620; Lam 2774, 2964; Posthumus 2408; Rachmat 48, 417; Riedel 9; Smith 644; Teijsmann HB 12252; Van Balgooy 3813,3904 ; Yusuf \& Wahyono 28

\section{MUSSAENDOPSIS}

Mussaendopsis celebica Bremek.

Sulawesi distribution - South

Altitude - 330-500 m

Collection(s) - Darnaedi 1884; De Vogel 5848, 6192, 6310; Kofman 279; Meijer 11182, 11204; Ramlanto 202; Van Balgooy 3702

\section{MYCETIA}

\section{Mycetia cauliflora Reinw.}

Sulawesi distribution - South

Lore Lindu National Park \& environs - present Altitude - 1000-1500 m

Collection(s) - Meijer 9492

\section{Mycetia spec.}

Sulawesi distribution - North, Central, South, South-East

Altitude - 50-1300 m

Collection(s) - Bloembergen 4111, 4175; Buwalda 3799; De Vogel 5673, 5737; De Vogel \& Vermeulen 6531; Elbert 3182; Hennipman 5968; Johansson 21; Milliken 866; Van Balgooy 3041, 3500

\section{MYRMECODIA}

\section{Myrmecodia tuberosa Jack}

Sulawesi distribution - North

Altitude - 80-1000 m

Collection(s) - Bloembergen 4070; Lam 2549

Myrmecodia spec.

Sulawesi distribution - Central, South

Lore Lindu National Park \& environs - present Altitude - 750-1500 m

Collection(s) - Meijer 9518; Van Balgooy 3520, 3574

\section{MYRMEPHYTUM}

Myrmephytum selebicum (Becc.) Becc.

Sulawesi distribution - South

Altitude $-400 \mathrm{~m}$

Collection(s) - Van Balgooy 3741 


\section{NAUCLEA}

\section{Nauclea orientalis L.}

Vernacular name(s) - Bangkala; Bantoeli Bantje; Lonkida

Sulawesi distribution - North, South, SouthEast

Altitude $-0-970 \mathrm{~m}$

Collection(s) - bb 5846, 23300, 23894, 32358, 32612; Cel I 38, IV 85; De Vriese \& Teijsmann 89; Elbert 2849, 3075; Forman 405; Ham R 6; Koorders 18700, 21226; Meijer 9942, 10819; Prawiroatmodjo \& Soewoko 1672; Teijsmann HB 41867

Nauclea subdita (Korth.) Steudel

Vernacular name(s) - Antalut'a

Sulawesi distribution - Central, South, SouthEast

Lore Lindu National Park \& environs - present Altitude - 10-1000 m

Collection(s) - bb 17154, 17629, 17645, 29325; Bloembergen 4067; Cel III 66; De Vogel 6266; Lam 2655; Prawiroatmodjo \& Maskuri 1161, 1181; Prawiroatmodjo \& Soewoko 1778; Ramlanto \& Fanani 706

Nauclea unicapitulifera Ridsdale

Sulawesi distribution - South-East

Altitude $-700 \mathrm{~m}$

Collection(s) - McDonald \& Ismail Cat 4035

\section{NEONAUCLEA}

\section{Neonauclea butonensis Ridsdale}

Sulawesi distribution - South-East

Collection(s) - Kjellberg 273

Neonauclea calycina (Bartl.) Merr.

Vernacular name(s) - Lobani poete

Sulawesi distribution - North, South, SouthEast

Altitude - 25-700 m

Collection(s) - Cel IV 117; De Vogel 5679; De Vogel \& Vermeulen 6495A; Meijer 11234, 11522; Prawiroatmodjo \& Maskuri 1536

Neonauclea celebica (Havil.) Merr.

Vernacular name(s) - Lebani [Soroako] Sulawesi distribution - North, Central, South, South-East
Altitude - 5-550 m

Collection(s) - bb 19410, 19601, 21736, 28847, 29464; Cel III 111; De Vogel 6072; Koorders 18627, 18641, 18644; Meijer 10794; Yoshida 1358

Neonauclea excelsa (Havil.) Merr.

Sulawesi distribution - South-East

Altitude - 20-250 m

Collection(s) - Prawiroatmodjo \& Soewoko 1791

\section{Neonauclea havilandii Merr.}

Vernacular name(s) - Bintawang

Sulawesi distribution - Central, South, SouthEast

Lore Lindu National Park \& environs - present Altitude - 0-1000 m

Collection(s) - Alston 16306; Cel I 79, II 492; De Vogel 5195, 5216, 5653, 5756; Elbert 3390; Johansson 180; Koorders 18624, 18629, 18630, 18632; Meijer 9741; Van Balgooy 3507

Neonauclea intercontinentalis Ridsdale

Sulawesi distribution - Central, South

Altitude $-800-1300 \mathrm{~m}$

Collection(s) - bb 24157; Meijer 9406

Neonauclea lanceolata Merr.

Sulawesi distribution - Central

Altitude - 1000-1250 m

Collection(s) - Hennipman 5613

Neonauclea pseudopeduncularis Ridsdale

Sulawesi distribution - North, Central, South Altitude - $1000 \mathrm{~m}$

Collection(s) - De Vogel 5095; Koorders 18643, 18699; Meijer 9533

\section{Neonauclea unicapitulifera Ridsdale}

Sulawesi distribution — South, South-East

Altitude - 0-700 m

Collection(s) - De Vogel 6163, 6305; Elbert 3392, 3477; Prawiroatmodjo \& Soewoko 1622; Widjaja 730

\section{Neonauclea ventricosa Ridsdale}

Vernacular name(s) - Lebano

Sulawesi distribution - North, Central, South 
Lore Lindu National Park \& environs - present Altitude - 200-1000 m

Collection(s) - bb 14119, 28238; Bloembergen 4120; De Vogel 6080; Koorders 18623; Meijer 10210; Noerkas 452; Ramlanto \& Fanani 777

\section{Neonauclea spec.}

Vernacular name(s) - Kundek kumdeh [Suwawa]

Sulawesi distribution - North, South

Altitude - $475 \mathrm{~m}$

Collection(s) - Meijer 11126A; Milliken 913

\section{NERTERA}

Nertera granadensis (Mutis ex L.f.) Druce

Sulawesi distribution - North, Central, South Altitude $-1300-2200 \mathrm{~m}$

Collection(s) - Forman 212; Koorders 19583; Meijer 9911; Monod de Froideville 129; Van Balgooy 3172

\section{OLDENLANDIA}

\section{Oldenlandia corymbosa L.}

Sulawesi distribution - Central, South, SouthEast

Altitude - 0-1000 m

Collection(s) - Bünnemeyer 10876, 11505, 12463; Elbert 2899

\section{Oldenlandia costata Koord.}

Sulawesi distribution - Central

Altitude - $350 \mathrm{~m}$

Collection(s) - Wardi Wd 092

\section{PAEDERIA}

\section{Paederia foetida L.}

Sulawesi distribution - North, Central, SouthEast

Altitude $-50-300 \mathrm{~m}$

Collection(s) - Elbert 2836; Kaudern 364; Kjellberg 116; Koorders 18663, 18664, 18665; Meijer 9217; Van Balgooy 2968; Widjaja 607

\section{Paederia spec.}

Sulawesi distribution - North, Central Lore Lindu National Park \& environs - present Altitude - 40-650 m
Collection(s) - Coode 5866; De Vogel 5022; Johansson 80

\section{PAVETTA}

Pavetta celebica Bremek.

Sulawesi distribution - South, South-East

Altitude - 0-1100 m

Collection(s) - Bünnemeyer 11815; Elbert 3419,3467

Pavetta makassarica Bremek.

Sulawesi distribution - South

Collection(s) - Teijsmann HB 12095

Pavetta spec.

Vernacular name(s) - Bungkudo hutan; Karinkawa [Buton]; Mogulantato

Sulawesi distribution - North, South, SouthEast

Altitude - 250-1000 m

Collection(s) - Bünnemeyer 11725; Coode 6284; McDonald \& Ismail Cat 4023; Milliken 909, 919; Rachmat 754; Ramlanto 236

\section{PORTERANDIA}

\section{Porterandia spec.}

Sulawesi distribution — South-East

Collection(s) - Coode 6113; Prawiroatmodjo \& Soewoko 1886

\section{PRARAVINIA}

Praravinia loconensis Bremek.

Sulawesi distribution - North

Altitude - 1000-1400 m

Collection(s) - De Vogel 2484; Koorders 18608, 18730,19219

\section{Praravinia minahassae Koord.}

Sulawesi distribution - North

Collection(s) - Koorders 18731

\section{Praravinia spec.}

Vernacular name(s) - Gora hutan

Sulawesi distribution - North, Central, South Altitude - 0-2355 m

Collection(s) - Bloembergen 3984; De Vogel 5128, 5129, 5294, 5438, 5443, 5444, 7267; 
De Vogel \& Vermeulen 6771, 6775, 7077; Forman 362; Hennipman 5162, 5516; Kjellberg 1975; Meijer 10078; Milliken 1056; Ramlanto \& Fanani 474; Van Balgooy 3149, 3266

\section{PSYCHOTRIA}

\section{Psychotria celebica Miq.}

Sulawesi distribution - North

Altitude $-350-750 \mathrm{~m}$

Collection(s) - Afriastini 2921; Burley, Partomihardjo \& Rachman 3503, 3542

Psychotria leptothyrsa Miq.

Sulawesi distribution - North

Collection(s) - Teijsmann HB 5779

Psychotria malayana Jack

Sulawesi distribution - South

Altitude $-200 \mathrm{~m}$

Collection(s) - Yusuf \& Wahyono 39

Psychotria viridiflora Reinw. ex Blume

Sulawesi distribution - South

Collection(s) - Afriastini 2074

Psychotria spec.

Vernacular name(s) - Talanggila hutan

Sulawesi distribution - North, Central, South, South-East

Altitude - 16-2350 m

Collection(s) - Chin 3583; Coode 5970, 6123, 6191, 6260A; De Joncheere 1104; De Vogel 5122, 5271, 5363, 5453, 5488, 5528, 5549, $5553,6132,6132 \mathrm{a}, 6142,6162,6164,6165$, 6263, 6299; De Vogel \& Vermeulen 6515, 6637, 6715, 6773, 7005, 7025; Kofman 230, 237; McDonald \& Ismail Cat 4053; Meijer 11270; Milliken 862, 916, 953, 1067, 1116; Wieringa 1842

\section{PSYDRAX}

Psydrax dicoccos Gaertn.

Sulawesi distribution - South

Altitude $-300 \mathrm{~m}$

Collection(s) - Cel V 289; Noerkas 225
RANDIA

Randia spec.

Sulawesi distribution - North, Central

Collection(s) - De Vogel 2633, 5118, 5616

\section{ROTHMANNIA}

Rothmannia forsteniana Miq.

Sulawesi distribution - North, Central, South Lore Lindu National Park \& environs - present

Collection(s) - Burley, Partomihardjo \& Rachman 4071; Johansson 396; Koorders 18594 , 18596, 18736; Lam 2433; Van Balgooy 3925

Rothmannia merrillii (Elmer) Ridsdale

Sulawesi distribution - South

Collection(s) - Van Balgooy 3894, 3946, 4082

\section{Rothmannia spec.}

Sulawesi distribution - North, Central, South, South-East

Collection(s) - Cel II 306, II 338, II 395; Coode 6023, 6027, 6201; Milliken 884; Prawiroatmodjo \& Soewoko 1919; Wieringa 1848

\section{SAPROSMA}

\section{Saprosma spec.}

Sulawesi distribution - Central

Lore Lindu National Park \& environs - present Altitude - 2000-2050 m

Collection(s) - De Vogel 5388, 5420

\section{SCHRADERA}

\section{Schradera spec.}

Sulawesi distribution - South

Altitude $-850 \mathrm{~m}$

Collection(s) - Yusuf \& Wahyono 131

\section{SCYPHIPHORA}

Scyphiphora hydrophyllacea Gaertn.

Sulawesi distribution - South, South-East

Altitude $-1 \mathrm{~m}$

Collection(s) - bb 19522; Kjellberg 526 
SPERMACOCE

Spermacoce articularis L.f.

Sulawesi distribution - South

Collection(s) - Rachmat 109

Spermacoce tenuior $\mathrm{L}$.

Sulawesi distribution - Central, South, SouthEast

Altitude - 0-280 m

Collection(s) - Bünnemeyer 10858; Elbert 3218; Kaudern 496

\section{SPIRADICLIS}

Spiradiclis caespitosa Blume

Sulawesi distribution - Central

Altitude $-250 \mathrm{~m}$

Collection(s) - Van Balgooy 3618

TARENNA

Tarenna forsteriana Miq.

Sulawesi distribution - North

Altitude $-700 \mathrm{~m}$

Collection(s) - Bogor Botanical Garden VD 18; Koorders 18703, 18704, 18705, 18733; Riedel HB 5909

Tarenna fragrans (Blume) Koord. \& Valeton

Sulawesi distribution - South

Altitude - $100 \mathrm{~m}$

Collection(s) - Afriastini 1983

Tarenna sambucina K. Schum \& Lauterb.

Sulawesi distribution - South

Collection(s) - Meijer 11104

Tarenna spec.

Sulawesi distribution - South, South-East Altitude - 20-1800 m

Collection(s) - bb 20427; Cel II 392; Chin 3579; Coode 6247; Darnaedi 2124; Hennipman 6054; Prawiroatmodjo \& Soewoko 1950; Teijsmann HB 13629; Van Balgooy 3839

\section{TARENNOIDEA}

Tarennoidea wallichii (Hook.f.) Tirveng.

Sulawesi distribution - North, South-East

Altitude - 200-1200 m

Collection(s) - bb 17179; McDonald \& Ismail Cat 4011

\section{TIMONIUS}

Timonius avenis Valeton

Sulawesi distribution - South

Altitude - 1000-1100 m

Collection(s) - Hennipman 6044

Timonius borneensis Valeton

Sulawesi distribution - North

Altitude $-400 \mathrm{~m}$

Collection(s) - Afriastini \& Rohajawati JJA 2909

\section{Timonius caudatifolius Elmer}

Sulawesi distribution - North

Altitude $-1000 \mathrm{~m}$

Collection(s) - De Vogel 2632

Timonius celebicus Koord.

Vernacular name(s) - Bambalud'a

Sulawesi distribution - North, South

Altitude $-0-600 \mathrm{~m}$

Collection(s) - bb 23893, 24491; Koorders 18686; Lam 2587; Ramlanto 151; Teijsmann HB 11739, HB 11952, HB 11985; Yusuf \& Wahyono 46

Timonius koordersii Valeton

Sulawesi distribution - North

Altitude $-500 \mathrm{~m}$

Collection(s) - Koorders 18745

Timonius minahassae Koord.

Sulawesi distribution - North, Central, South Altitude - 200-1000 m

Collection(s) - Burley, Partomihardjo \& Rachman 3513; Darnaedi 1759; Koorders 18739, 18742, 17188; Meijer 11312; Ramlanto \& Fanani 477 
Timonius schumannii Koord. ex Valeton

Sulawesi distribution - North

Altitude - 350-500 m

Collection(s) - De Vriese \& Teijsmann 100; Koorders 18684, 18711, 18712

Timonius stipularis Boerl.

Sulawesi distribution - South

Altitude $-200 \mathrm{~m}$

Collection(s) - Afriastini 2051

Timonius subsessilis Valeton

Sulawesi distribution - Central

Lore Lindu National Park \& environs - present

Altitude $-900 \mathrm{~m}$

Collection(s) - Meijer 10207

Timonius teysmannii Valeton

Sulawesi distribution - South

Collection(s) - Teijsmann HB 13744, HB 13992, HB 14004, HB 14060

\section{Timonius spec.}

Vernacular name(s) - Ike [Besoa]; Jambu monyet hutan; Kolomioe, Golomio; Lembanu tupu [Besoa]; Para ala; Uampara [Soroako]

Sulawesi distribution - North, Central, South, South-East

Altitude - 0-1500 m

Collection(s) - Afriastini 2070; bb 17978, 26289; Cel II 219, II 12460, V 214; Chin 3639; Coode 5890; De Vogel 5547, 5562, 5575, 5719, 5955, 6012, 6077, 6241, 6366; De Vogel \& Vermeulen 6772, 6890, 6974, 7082; Elbert 2570, 2740, 3137; Hennipman 5669, 5828, 6045; Johansson 185, 335, 597; Koorders 19117; McDonald \& Ismail Cat 4171; Meijer 9721, 10002, 11275; Milliken 878, 936; Noerkas 415; Prawiroatmodjo \& Soewoko 1803; Ramlanto 117; Riedel HB 5911; Van Balgooy 3053, 3738, 3763, 3799, 3814, 4012; Whitmore \& Sidiyasa TCW 3397, TCW 3502; Widjaja 727

\section{TRICALYSIA}

\section{Tricalysia minahasae Koord.}

Sulawesi distribution - North, Central

Altitude - 200-1000 m

Collection(s) - Church \& Ismail 2; Tantra 1506
Tricalysia singularis (Blume) Boerl.

Sulawesi distribution - North

Altitude $-400 \mathrm{~m}$

Collection(s) - Burley, Partomihardjo \& Rachman 3593

\section{UNCARIA}

Uncaria acida Roxb.

Sulawesi distribution - South

Altitude - $500 \mathrm{~m}$

Collection(s) - Van Balgooy 3869

Uncaria attenuata Korth.

Sulawesi distribution - North

Altitude $-600 \mathrm{~m}$

Collection(s) - Kaudern 100, 264

Uncaria cordata (Lour.) Merr. var. cordata

Sulawesi distribution - South

Altitude - 100-600 m

Collection(s) - De Vogel 6022, 6056; Van Balgooy 3784

Uncaria lanosa Wall. appendiculata (Benth.) Ridsdale

Sulawesi distribution - North, South, SouthEast

Altitude $-20-700 \mathrm{~m}$

Collection(s) - Elbert 3045; Koorders 18706, 18707; Lam 2621; Meijer 11311; Prawiroatmodjo \& Soewoko 1693; Rachmat 418

Uncaria lanosa Wall. forma philippinensis Ridsdale

Sulawesi distribution - North

Altitude $-300 \mathrm{~m}$

Collection(s) - De Vogel \& Vermeulen 7023

Uncaria longiflora (Miq.) Merr.

Vernacular name(s) - Genggáil'a

Sulawesi distribution - North

Altitude $-60 \mathrm{~m}$

Collection(s) - Lam 2825

UROPHYLLUM

Urophyllum arboreum Korth.

Sulawesi distribution - Central 
Lore Lindu National Park \& environs - present

Altitude $-2250 \mathrm{~m}$

Collection(s) - Tantra 1574

Urophyllum glabrum Wall.

Sulawesi distribution - Central

Altitude - $900 \mathrm{~m}$

Collection(s) - Ramlanto \& Fanani 540

Urophyllum macrophyllum Korth.

Sulawesi distribution - Central, South-East

Altitude - 100-300 m

Collection(s) - Amir 137; Prawiroatmodjo \& Maskuri 1242, 1434, 1454, 1509; Ramlanto \& Fanani 736

\section{Urophyllum spec.}

Sulawesi distribution - North, Central, South, South-East

Altitude - 20-2000 m

Collection(s) - De Vogel 5156, 5291, 5348, 5417; Meijer 9546; Milliken 968, 1110; Prawiroatmodjo \& Soewoko 1991; Van Balgooy 3050, 3166, 3167; Wieringa 1863

\section{WENDLANDIA}

Wendlandia densiflora (Blume) DC. forma rufescens (Miq.) Bakh.f.

Sulawesi distribution - South

Altitude - 950-1150 m

Collection(s) - Bünnemeyer 11042, 11816A; Cel 4

Wendlandia paniculata Roxb.

Sulawesi distribution - South-East

Collection(s) - Widjaja 692

\section{Wendlandia spec.}

Vernacular name(s) - Bingkaramo [Besoa]; Poei

Sulawesi distribution - North, Central, South, South-East

Altitude $-0-2050 \mathrm{~m}$

Collection(s) - Alston 16067; bb 14120, 26270; De Vogel 5273; Elbert 2626; Hennipman 5763, 5839; Noerkas 229; Rachmat 82, 384; Yoshida 1122

\section{RUTACEAE}

ACRONYCHIA

Acronychia pedunculata Miq.

Sulawesi distribution - South, South-East

Collection(s) - Elbert 3465; Ramlanto 169

Acronychia trifoliolata Zoll. \& Moritzi

Vernacular name(s) - batjaena alae

Sulawesi distribution - South

Altitude $-1800 \mathrm{~m}$

Collection(s) - bb 26292; Van Vuuren 279

Acronychia spec.

Sulawesi distribution - Central, South

Collection(s) - De Vogel 6125, 6178, 6184;

Tantra 1586; Van Balgooy 3254, 3644, 3955

\section{AEGLE}

Aegle marmelos (L.) Corrêa

Sulawesi distribution - South

Collection(s) - Van Vuuren 307

\section{BOENNINGHAUSENIA}

Boenninghausenia albiflora Rchb.

Sulawesi distribution - North, South

Lore Lindu National Park \& environs - present

Collection(s) - Bloembergen 3965; Bünnemeyer 12308; Meijer 9919

\section{CITRUS}

\section{Citrus aurantium L.}

Sulawesi distribution - South

Lore Lindu National Park \& environs — present Altitude $-1000 \mathrm{~m}$

Collection(s) - Meijer 9549

Citrus histrix DC.

Sulawesi distribution - South-East

Altitude - 0-200 m

Collection(s) - Elbert 2605, 3368

Citrus maxima (Burm.f.) Merr.

Vernacular name(s) - Lemo

Sulawesi distribution - Central 
Altitude $-950 \mathrm{~m}$

Collection(s) - Bünnemeyer 10989

Citrus spec.

Vernacular name(s) - Muncu [Uma]

Sulawesi distribution - Central, South

Altitude - 250-850 m

Collection(s) - Meijer 10749; Van Balgooy 3584, 3585; Yusuf \& Wahyono 151

\section{CLAUSENA}

\section{Clausena excavata Burm.f.}

Sulawesi distribution - North, South, SouthEast

Altitude - 0-250 m

Collection(s) - Elbert 2979; Koorders 18748, 18749; Prawiroatmodjo \& Soewoko 1625, 1681; Ramlanto 124

\section{GLYCOSMIS}

Glycosmis angustata Tanaka

Sulawesi distribution - South

Collection(s) - Teijsmann HB 13595, HB 14219

\section{Glycosmis cochinchinensis Pierre}

Sulawesi distribution - North

Altitude - $140 \mathrm{~m}$

Collection(s) - De Vogel 2566

Glycosmis cyanocarpa (Blume) Spreng. var. platyphylla (Merr.) Tanaka

Vernacular name(s) - Suwi

Sulawesi distribution - North

Altitude - 40-90 m

Collection(s) - Lam 2985, 3354

Glycosmis sapindoides Lindl. ex Wall. var. gracilis Tanaka

Sulawesi distribution - South

Collection(s) - Teijsmann HB 11794, HB 12283

\section{LUNASIA}

\section{Lunasia amara Blanco}

Sulawesi distribution - North, Central, South, South-East
Collection(s) - Coode 5847, 6239, 6241; De Vogel \& Vermeulen 6594; Hennipman 5763; Meijer 11121; Van Balgooy 3677; Whitmore \& Sidiyasa TCW 3427

\section{Lunasia amara Blanco var. amara}

Sulawesi distribution - North, Central, South, South-East

Altitude - 0-900 m

Collection(s) - bb 19602; Cel V 271, V 276; Elbert 3238; Koorders 16946, 16948, 16953, 18756, 18761; Lam 2661, 3181, 3291, 3305; Reinwardt 1586

\section{MELICOPE}

Melicope bonwickii (F. Muell.) T.G. Hartley

Sulawesi distribution - Central

Collection(s) - Tantra 1514

Melicope celebica T.G. Hartley

Sulawesi distribution - South

Altitude $-200 \mathrm{~m}$

Collection(s) - Cel 2371

Melicope confusa (Merr.) Liu

Vernacular name(s) - ampak; maratalam'a; matalang'a

Sulawesi distribution - North, South, North

Altitude $-1500 \mathrm{~m}$

Collection(s) - bb 24086, 24511, 32505, 33091; De Vriese \& Teijsmann 123; Koorders $18758,18759,18760,18762,18765,18766$, 18767, 18777; Lam 2821, 2974; Meijer 10866; Van Vuuren 427

Melicope latifolia (DC.) T.G. Hartley

Sulawesi distribution - North, South

Altitude $-0-800 \mathrm{~m}$

Collection(s) - bb 18638; Forsten 370; Koorders 16950, 18763, 18764, 18768, 18769, 18770; Lam 2506, 2576; Van Vuuren 350

Melicope maliliensis T.G. Hartley

Vernacular name(s) - Hokio; Popaya

Sulawesi distribution - South

Altitude $-200 \mathrm{~m}$

Collection(s) - Cel II 356, II 488 
Melicope triphylla (Lam.) Merr.

Vernacular name(s) - arangijoh

Sulawesi distribution - North, South

Altitude - 0-850 m

Collection(s) - Bloembergen 4041; Forman 361; Koorders 19221; Lam 2533, 2869; Teijsmann HB 5323; Yusuf \& Wahyono 136

\section{Melicope spec.}

Sulawesi distribution - North, Central, South, South-East

Collection(s) - Alston 15664; De Vogel 5521; De Vogel \& Vermeulen 6718, 6756, 6931; Meijer 9953, 10712; Prawiroatmodjo \& Soewoko 1624; Tantra 1591; Van Balgooy $3444,3899,3937,3994$; Yusuf \& Wahyono 55,129

\section{MICROMELUM}

Micromelum minutum (G. Forst.) Wight \& Arn.

Vernacular name(s) - Boerese

Sulawesi distribution - North, Central, South, South-East

Altitude - 20-1600 m

Collection(s) - bb 19700, 26299; Cel I 62; De Vogel 2526, 5763, 5787; Koorders 18771; Meijer 10180; Prawiroatmodjo \& Soewoko 1616; Teijsmann HB 13826; Van Balgooy 2999; Van Vuuren 94

\section{Micromelum spec.}

Sulawesi distribution - Central

Lore Lindu National Park \& environs - present Altitude $-1200 \mathrm{~m}$

Collection(s) - De Vogel 5279

MURRAYA

Murraya paniculata (L.) Jack

Vernacular name(s) - Dinggato

Sulawesi distribution - North, Central

Altitude - 10-160 m

Collection(s) - bb 15390, 31496; Koorders 18917

\section{Murraya spec.}

Sulawesi distribution - North

Altitude $-100 \mathrm{~m}$

Collection(s) - bb 17046

\section{TETRACTOMIA}

Tetractomia barringtonioides T.G. Hartley

Vernacular name(s) - Hokiowoetemia; Moro kandoa

Sulawesi distribution - South

Altitude $-2-200 \mathrm{~m}$

Collection(s) - Cel II 361, III 30

Tetractomia tetrandra (Roxb.) Merr.

Vernacular name(s) - Ponto-alo; Popaya poete; Taoesape

Sulawesi distribution - North, South

Lore Lindu National Park \& environs - present

Altitude - 10-1600 m

Collection(s) - bb 24168; Bloembergen 3944; Cel II 321, II 483; Ramlanto 130

\section{Tetractomia spec.}

Sulawesi distribution - Central, South

Altitude - 150-1700 m

Collection(s) - De Vogel 6140, 6161, 6226, 6240; Van Balgooy 3298, 3689, 3897, 4039

TETRADIUM

Tetradium glabrifolium (Champ. ex Benth.)

T.G. Hartley

Sulawesi distribution-Central, South, SouthEast

Lore Lindu National Park \& environs - present

Collection(s) - Coode 6100; De Vogel 5033, 5167; Meijer 9301, 9466

Tetradium sambucinum (Blume) T.G. Hartley

Sulawesi distribution - Central

Collection(s) - Tantra 1501

Tetradium spec.

Sulawesi distribution - North

Collection(s) - De Vogel \& Vermeulen 6832

\section{TODDALIA}

\section{Toddalia asiatica Lam.}

Sulawesi distribution - North

Collection(s) - Alston 15913 


\section{ZANTHOXYLUM}

Zanthoxylum avicennae (Lam.) DC.

Vernacular name(s) - Marimbos'a

Sulawesi distribution - North

Altitude - $300 \mathrm{~m}$

Collection(s) — Lam 3254

\section{Zanthoxylum celebicum Koord.}

Sulawesi distribution - North

Altitude - 0-100 m

Collection(s) — bb 19450; Koorders 18746

\section{Zanthoxylum limonella (Dennst.) Alston}

Sulawesi distribution - North, South

Altitude $-50-800 \mathrm{~m}$

Collection(s) - bb 18418; Koorders 18773, 18775; Van Vuuren 154

Zanthoxylum nitidum (Roxb.) DC

Sulawesi distribution - South-East

Altitude $-150-300 \mathrm{~m}$

Collection(s) - Elbert 3445

\section{Zanthoxylum spec.}

Sulawesi distribution - North, Central Lore Lindu National Park \& environs - present

Collection(s) - De Vogel 5017; Van Balgooy 3547

\section{SABIACEAE}

\section{MELIOSMA}

Meliosma lanceolata Blume var. lanceolata

Sulawesi distribution - North

Altitude $-150 \mathrm{~m}$

Collection(s) - Burley, Partomihardjo \& Rachman 4078

Meliosma nitida Blume

Sulawesi distribution - North

Altitude $-300 \mathrm{~m}$

Collection(s) - Afriastini \& Rohajawati JJA 2939
Meliosma pinnata (Roxb.) Walp. subsp. macrophylla (Merr.) Beusekom

Sulawesi distribution - North

Altitude - 220-700 m

Collection(s) - bb 14135; De Vogel 6720; Forman 266; Koorders 18783, 18785, 18786, 18787, 18789

Meliosma pinnata (Roxb.) Walp. subsp. sylvatica (Elmer) Beusekom

Sulawesi distribution - South

Altitude $-2000 \mathrm{~m}$

Collection(s) - Sands 383

Meliosma sumatrana (Jack) Walp.

Sulawesi distribution - North, Central, South, South-East

Altitude $-1100 \mathrm{~m}$

Collection(s) - Amir 240; bb 14222, 17200, 18083, 18610, 18678, 28237; Coode 5968; De Vogel 2608, 5071, 5263, 5576; De Vogel \& Vermeulen 6690, 6985, 7157; De Vriese 326; Johansson 389; Keßler et al. PK 3132 , PK 3158; Koorders 18778, 18779, 18780 , 18781, 18782, 18784, 18790, 18791, 18792; Meijer 9306, 9361A, 9527; Milliken 905, 942; Prawiroatmodjo \& Soewoko 1969; Ramlanto \& Fanani 473; Van Balgooy 3098, 3516; Whitmore \& Sidiyasa TCW 3426; Yusuf \& Wahyono 61

\section{Meliosma spec.}

Sulawesi distribution - North, Central, South, South-East

Altitude - 70-1500 m

Collection(s) - Burley, Partomihardjo \& Rachman 3913; Coode 5968; De Vogel 5540, 6041, 6112, 6215; De Vogel \& Vermeulen 6720, 7068; McDonald \& Ismail 4059

\section{SABIA}

\section{Sabia spec.}

Vernacular name(s) - Walatida [Kulawi]

Sulawesi distribution - Central

Lore Lindu National Park \& environs - present Altitude - 650-1000 m

Collection(s) - De Vogel 5506; Johansson 67, 528 


\section{SALVADORACEAE}

AZIMA

Azima sarmentosa Benth. \& Hook.f.

Vernacular name(s) - Walaboengga

Sulawesi distribution - Central, South

Lore Lindu National Park \& environs - present Altitude $-25 \mathrm{~m}$

Collection(s) - bb 20; Meijer 9206; Teijsmann HB 13905, HB 14103

\section{SANTALACEAE}

\section{SANTALUM}

\section{Santalum album L.}

Sulawesi distribution - Central

Lore Lindu National Park \& environs - present Altitude $-200 \mathrm{~m}$

Collection(s) - bb 17231; Meijer 9187

\section{SAPINDACEAE}

\section{ALECTRYON}

\section{Alectryon glaber Radlk.}

Sulawesi distribution - North, Central, South Altitude - 200-1000 m

Collection(s) - Alston 16075; De Vogel 5031, 5754; Koorders 18817; Meijer 10211; Tantra 1511; Van Balgooy 2990

\section{ALLOPHYLUS}

Allophylus cobbe (L.) Raeusch.

Vernacular name(s) - Belante [Besoa]

Sulawesi distribution - North, Central, South, South-East

Lore Lindu National Park \& environs - present Altitude $-1000 \mathrm{~m}$

Collection(s) - Alston 16101; Chin 3543; Coode 5799; De Vogel 5507, 5537; Elbert 2510, 2558; Eyma 3534, 4208; Forsten 6, 8, 39; Kaudern 203; Koorders 18831; Lam 2505, 3378, 3428; Meijer 10107; Tantra 1512; Teijsmann HB 11867; Van Balgooy 3504; Van Vuuren 192

\section{ARYTERA}

Arytera litoralis Blume

Vernacular name(s) - Karope

Sulawesi distribution - North, Central, South, South-East

Altitude $-0-400 \mathrm{~m}$

Collection(s) - bb 4182, 24990; Cel V 142; De Vogel 5663, 5768; Elbert 3406; Prawiroatmodjo \& Soewoko 1782; Teijsmann HB 12276, HB 12535, HB 12868, HB 14163

\section{CARDIOSPERMUM}

\section{Cardiospermum halicacabum L.}

Sulawesi distribution - Central, South, SouthEast

Lore Lindu National Park \& environs - present Altitude - 0-900 m

Collection(s) — Bünnemeyer 11655, 12573; De Joncheere 1027; De Vogel 5041; Elbert 2634, 3355; Eyma 1138; Hennipman 5019; Kaudern 53; Van Vuuren 79; Widjaja 662, 707; Yoshida 887

\section{CUBILIA}

Cubilia cubili (Blume) Adelb.

Sulawesi distribution - North, South

Altitude - 20-1700 m

Collection(s) - bb 32800; Cel I 8, V 236; Koorders 18841, 18845, 18846, 18847, $18850,22616,29487$

\section{CUPANIOPSIS}

\section{Cupaniopsis celebica Adema}

Sulawesi distribution - South

Altitude - 30-200 m

Collection(s) - Cel II 369, IV 84

\section{Cupaniopsis strigosa Adema}

Sulawesi distribution - South Altitude $-2000 \mathrm{~m}$

Collection(s) - bb 5461 


\section{DICTYONEURA}

Dictyoneura acuminata Blume subsp. acuminata

Sulawesi distribution - South

Altitude $-450 \mathrm{~m}$

Collection(s) - De Vogel 6286

\section{DIMOCARPUS}

\section{Dimocarpus angustifolia L.f.}

Sulawesi distribution - South

Altitude $-2000 \mathrm{~m}$

Collection(s) - Bünnemeyer 12093; Meijer 11049; Yoshida 995, 1376

\section{Dimocarpus longan Lour. var. malesianus} Leenh.

Sulawesi distribution - North, South-East Altitude $-50-750 \mathrm{~m}$

Collection(s) - bb 19432, 21771; De Vogel \& Vermeulen 6807

\section{DODONAEA}

\section{Dodonaea viscosa Jacq.}

Sulawesi distribution - North

Collection(s) - bb 16991; Koorders 18818;

Reinwardt 1696; Van Vuuren 343

\section{ELATTOSTACHYS}

\section{Elattostachys erythrocarpum Adema}

Sulawesi distribution - North

Altitude $-50 \mathrm{~m}$

Collection(s) - Eyma 1778

Elattostachys verrucosa (Blume) Radlk.

Vernacular name(s) - Padja-padja

Sulawesi distribution - South

Altitude $-1200 \mathrm{~m}$

Collection(s) - bb 5158

Elattostachys zippeliana (Blume) Radlk.

Vernacular name(s) - Ikoeli maeto molaka

Sulawesi distribution - North, South

Altitude $-0-250 \mathrm{~m}$

Collection(s) - bb 17180; Cel V 207; De Vriese 55, 60; De Vriese \& Teijsmann 23; Koorders
18820; Meijer 10844; Teijsmann HB 11730 , HB 11757, HB 12294

\section{EUPHORIANTHUS}

Euphorianthus euneurus Leenh.

Vernacular name(s) - Kalo kaloro; Polohu [Besoa]; Roroensoe

Sulawesi distribution - North, Central, South Lore Lindu National Park \& environs - present Altitude - 0-1050 m

Collection(s) - bb 28252; Cel I 77, III 58; De Vogel 5601; Koorders 18839, 18848; Meijer 11231; Prawiroatmodjo \& Soewoko 1738; Van Balgooy 3084

\section{GANOPHYLLUM}

\section{Ganophyllum falcatum Blume}

Sulawesi distribution - Central, South, SouthEast

Lore Lindu National Park \& environs - present Altitude - 30-400 m

Collection(s) - bb 21772, 22620, 31854; Cel V 222; Meijer 10853

\section{GUIOA}

Guioa diplopetala (Hassk.) Radlk.

Vernacular name(s) - Sosoöerioe

Sulawesi distribution - South, South-East

Altitude - 3-70 m

Collection(s) - Amir 98, 209; Cel IV 91, IV 181; Ramlanto 223

\section{Guioa hirsuta Welzen}

Sulawesi distribution - South

Altitude $-400-425 \mathrm{~m}$

Collection(s) - De Vogel 6202; Van Balgooy 3658

\section{HARPULLIA}

\section{Harpullia arborea Radlk.}

Sulawesi distribution - North, Central, South, South-East

Altitude - 0-1000 m

Collection(s) - bb 21706; Cel V 154; De Vogel 2534; De Vogel \& Vermeulen 6652; Elbert 3261; Koorders 18842; Van Balgooy 3119; Whitmore \& Sidiyasa TCW 3529 
Harpullia arborea Radlk. subsp. arborea

Sulawesi distribution - Central

Altitude - 1000-1200 m

Collection(s) - Van Balgooy 3058, 3491

Harpullia cupanioides Roxb.

Vernacular name(s) - Karoma

Sulawesi distribution - Central, South

Altitude $-200-1000 \mathrm{~m}$

Collection(s) - Cel I 69; Meijer 9248; Van Balgooy 3539

\section{LEPIDOPETALUM}

\section{Lepidopetalum spec.}

Sulawesi distribution - South

Collection(s) - Teijsmann HB 11745, HB 11760

\section{LEPISANTHES}

Lepisanthes fruticosa (Roxb.) Leenh.

Sulawesi distribution - North, South, SouthEast

Altitude $-0-300 \mathrm{~m}$

Collection(s) - Chin 3424; Elbert 2676, 2894, 2934; Eyma 4210; Koorders 18821, 18822; Meijer 10651; Noerkas 429; Rachmat 794; Whitmore \& Sidiyasa TCW 3466

Lepisanthes rubiginosa (Roxb.) Leenh.

Vernacular name(s) - Madansat

Sulawesi distribution - North, South, SouthEast

Altitude - 0-645 m

Collection(s) - bb 14141; Bünnemeyer 12532a; Elbert 2604, 2796, 3014, 3022, 3070, 3246, 3360, 3434; Forman 239, 329; Meijer 10659; Noerkas 300; Rachmat 630; Teijsmann HB 13933

Lepisanthes senegalensis (Poir.) Leenh.

Sulawesi distribution - North, South

Altitude $-0-50 \mathrm{~m}$

Collection(s) - Koorders 18015; Teijsmann HB 12763, HB 12891

\section{LITCHI}

Litchi chinensis Sonn.

Sulawesi distribution - Central

Lore Lindu National Park \& environs — present Altitude $-2000 \mathrm{~m}$

Collection(s) - Tantra 1606

\section{MISCHOCARPUS}

\section{Mischocarpus sundaicus Blume}

Sulawesi distribution - North, South

Altitude $-1000 \mathrm{~m}$

Collection(s) - bb 17681; Teijsmann HB 12404, HB 13608, HB 13711, HB 14061

\section{NEPHELIUM}

Nephelium lappaceum L. var. pallens Leenh.

Vernacular name(s) - Walitoe mongkoeni Sulawesi distribution - South, South-East

Altitude - 10-400 m

Collection(s) - bb 6040, 20893, 21735, 31819;

Cel IV 102, IV 169, IV 170, IV 171, V 196;

Meijer 11223

Nephelium ramboutan-ake (Labill.) Leenh.

Sulawesi distribution - North, Central

Altitude $-220-350 \mathrm{~m}$

Collection(s) - Burley, Partomihardjo \& Rachman 3527; De Vogel \& Vermeulen 6719, 6737; Ramlanto \& Fanani 640; Whitmore \& Sidiyasa TCW 3419

\section{POMETIA}

Pometia pinnata J.R. Forst. \& G. Forst.

Sulawesi distribution - North, South, SouthEast

Altitude $-20-500 \mathrm{~m}$

Collection(s) - bb 25537; De Vogel 6665; Koorders 18823, 18825, 18827; Prawiroatmodjo \& Soewoko 1964

Pometia pinnata J.R. Forst. \& G. Forst. forma glabra (Blume) M. Jacobs

Vernacular name(s) - Boetongali; Ilase mongkoeni; Lutut'a

Sulawesi distribution - North, Central, South

Altitude $-30-600 \mathrm{~m}$ 
Collection(s) - bb 15707, 22992, 28234, 31491, 32464, 32523, 32616; Cel V 227; Koorders 18824; Lam 2852; Van Vuuren 434

Pometia pinnata J. R. Forst. \& G. Forst. forma pinnata

Sulawesi distribution - Central, South-East

Altitude - $15 \mathrm{~m}$

Collection(s) - bb 28736

Pometia pinnata J.R. Forst. \& G. Forst. forma repanda $M$. Jacobs

Vernacular name(s) - Gehe; Ilase poeti; Ilase tamoengkoe; Leo; Woesel

Sulawesi distribution - North, South

Altitude $-40-700 \mathrm{~m}$

Collection(s) - bb 19417, 32480, 33117; Cel 239, V 231; Forman 320; Koorders 18826; Lam 2982, 3128

\section{RHYSOTOECHIA}

Rhysotoechia koordersii Radlk.

Sulawesi distribution - South, North

Altitude $-20-700 \mathrm{~m}$

Collection(s) - Cel III 180; Koorders 18844

Rhysotoechia spec.

Sulawesi distribution - South

Altitude $-400 \mathrm{~m}$

Collection(s) - De Vogel 5721

SCHLEICHERA

Schleichera oleosa (Lour.) Oken

Vernacular name(s) - Ading/Adjpe sompi

Sulawesi distribution - South, South-East

Altitude - 0-200 m

Collection(s) - bb 20253; Elbert 3310; Van Vuuren $78 \mathrm{a}$

\section{TRIGONACHRAS}

Trigonachras celebensis Leenh.

Vernacular name(s) - Woelisie

Sulawesi distribution - Central, South, SouthEast

Altitude - 20-300 m

Collection(s) - Cel II 400; Grimes 1180; Prawiroatmodjo \& Soewoko 1747

\section{TRISTIRA}

Tristira triptera (Blanco) Radlk.

Vernacular name(s) - Marasigo

Sulawesi distribution - Central, South, SouthEast

Altitude - 0-200 m

Collection(s) - bb 21768; Cel I 41; Koorders 18840; Meijer 10174; Teijsmann HB 13756, HB 13809

\section{TRISTIROPSIS}

Tristiropsis acutangula Radlk.

Sulawesi distribution - South-East

Altitude $-100 \mathrm{~m}$

Collection(s) - bb 21774

\section{SAPOTACEAE}

\section{CHRYSOPHYLLUM}

\section{Chrysophyllum roxburghii G. Don}

Vernacular name(s) - Dondongisilalcino [Tobela]

Sulawesi distribution - Central, South, SouthEast

Altitude - 10-300 m

Collection(s) - bb 21333; Cel V 192; Ramlanto \& Fanani 673

\section{MADHUCA}

Madhuca burckiana (Koord.) H.J. Lam

Vernacular name(s) - Dama dere [Soroako]

Sulawesi distribution - North, South

Altitude $-0-400 \mathrm{~m}$

Collection(s) - De Vogel 61 15, 6185; Koorders 18883

\section{MANILKARA}

\section{Manilkara celebica H.J. Lam}

Vernacular name(s) - Timboeolo

Sulawesi distribution - North, Central

Altitude $-50-300 \mathrm{~m}$

Collection(s) - bb 16979, 19401, 19637, 30152, $31489,31840,31885,32365,32528$ 
Manilkara fasciculata (Warb.) H.J. Lam \& Maas Geest.

Vernacular name(s) - Koemia batoe

Sulawesi distribution - North, Central, South Altitude - 100-500 m

Collection(s) — bb 31519, 31835, 31881, 32599

\section{MIMUSOPS}

\section{Mimusops elengi $L$.}

Vernacular name(s) - Kariskis; Molosiggo; Tandjoeng; Toto

Sulawesi distribution - North, Central, South, South-East

Altitude $-0-300 \mathrm{~m}$

Collection(s) - Alston 16082; bb 3900, 13743, $17038,17211,19395,19638,31487,31833$, $31860,31889,32364,32488,32527$; Coode 5909; B. Heyne 2451; Kjellberg 529; Meijer 10689; Teijsmann HB 11828, HB 12241, HB 12309

\section{PALAQUIUM}

\section{Palaquium amboinense Burck}

Vernacular name(s) - Nato

Sulawesi distribution - Central, South

Altitude - 5-100 m

Collection(s) - bb 31853, 31862, 33079

Palaquium luzoniense (Fern.-Vill.) Vidal

Sulawesi distribution - South

Altitude $-100 \mathrm{~m}$

Collection(s) - bb 1855; Cel II 474

Palaquium maliliense P. Royen

Vernacular name(s) - Koeme; Polloetan

Sulawesi distribution - North, South, SouthEast

Altitude $-0-400 \mathrm{~m}$

Collection(s) - bb 11417, 23253, 31830, 32465, 33118; Cel 474, II 495; Lam 2736; Meijer 11144

Palaquium obovatum (Griff.) Engl.

Sulawesi distribution - North, South

Collection(s) - Meijer 10700, 10732, 10841, 11506
Palaquium obovatum (Griff.) Engl. var. orientale H.J. Lam

Vernacular name(s) - Damar natoh; Kume [Uma]; Loengkap

Sulawesi distribution - North, Central, South, South-East

Altitude - 5-1000 m

Collection(s) - bb 17002, 17639, 20891, 20901, 21313, 21351, 28214, 28725, 31839 , 32473; Bogor Botanical Garden HB 5358; De Vriese 17, 34; De Vriese \& Teijsmann 6; Koorders 18859, 18862, 18863, 18891; Meijer 9738; Noerkas 431; Van Balgooy 3529

\section{Palaquium obtusifolium Burck}

Vernacular name(s) - Bokoroe; Koeme mopoete; Nantoe; Tagimanoe

Sulawesi distribution - North, Central, South, South-East

Altitude $-3-800 \mathrm{~m}$

Collection(s) - bb 5564, 5854, 7516, 8451, $19413,22958,23261,24505,28732,31523$, 31836, 31843, 31848, 31905, 32481, 32606; Burley, Partomihardjo \& Rachman 3624; Cel II 308, II 427; Koorders 18858, 18861, 18887, 18888; Lam 3006, 3355, 3439; Meijer 9411; Prawiroatmodjo \& Soewoko 1848

Palaquium quercifolium (de Vriese) Burck

Sulawesi distribution - South

Collection(s) — De Vriese 1, 4

Palaquium ridleyi King \& Gamble

Sulawesi distribution - South

Altitude $-400-500 \mathrm{~m}$

Collection(s) - Van Balgooy 3949, 4062

Palaquium rostratum (Miq.) Burck

Sulawesi distribution - North, South-East

Altitude - 25-270 m

Collection(s) - bb 17130, 21710

\section{Palaquium spec.}

Vernacular name(s) - Kandole; Paraäla laai [Tobala]

Sulawesi distribution - North, Central, South, South-East

Altitude - 20-1100 m 
Collection(s) - bb 31509, 31672, 31822, 31873, 31917, 32544; Cel II 372; Coode 6122; De Vogel 6398; Meijer 10149

\section{PLANCHONELLA}

Planchonella duclitan (Blanco) Bakh.f.

Sulawesi distribution - North, Central, South, South-East

Altitude $-0-500 \mathrm{~m}$

Collection(s) - bb 17040, 21092, 21311, 29469; Cel V 148; De Vriese 44; Koorders 18868, 18875, 18879, 18880, 18881; Teijsmann HB 5259, HB 12400, HB 14054

Planchonella firma (Miq.) Dubard var. firma

Vernacular name(s) - Bakko-bakko/Koeme Wa [Boegineesch/Tobelia']

Sulawesi distribution - South

Altitude $-100 \mathrm{~m}$

Collection(s) - Cel II 309, II 380

Planchonella firma (Miq.) Dubard var. microcarpa (Burck) H.J. Lam

Vernacular name(s) - Alewat'a; Bitawak a batoe; Goemat'oe Sangita

Sulawesi distribution - North

Altitude - 180-1100 m

Collection(s) - bb 15028; Koorders 15952, 18877; Lam 2923, 2924, 3099

Planchonella linggensis (Burck) Pierre

Vernacular name(s) - nakimpasaan; pae-pae [Tobela]; to pado [Tobela]

Sulawesi distribution - North, South, SouthEast

Altitude - 4-350 m

Collection(s) - bb 19402, 21763; Cel II 382, II 682, IV 99; Church \& Ismail 11; Lam 3311

Planchonella linggensis (Burck) Pierre var. linggensis

Vernacular name(s) - morolemo [Tobela]; morolemo mapoete [Tobela]

Sulawesi distribution - South

Altitude $-100 \mathrm{~m}$

Collection(s) - Cel II 355, II 462

Planchonella macropoda H.J. Lam

Sulawesi distribution - Central

Lore Lindu National Park \& environs — present
Altitude $-2000 \mathrm{~m}$

Collection(s) - Tantra 1584

Planchonella obovata (R.Br.) Pierre

Vernacular name(s) - laladan; lolotan

Sulawesi distribution - North, Central, South, South-East

Altitude - 5-400 m

Collection(s) - Alston 16078; bb 1833, 1837, 1918, 21585, 21712; Cel III 42; Koorders 18867; Lam 3272, 3447; Meijer 9275; Widjaja 737

Planchonella obovoidea H.J. Lam

Sulawesi distribution - South-East

Altitude - 250-320 m

Collection(s) - bb 31285; Elbert 2747, 6505, 6509,6510

Planchonella spec.

Sulawesi distribution - South

Altitude - $430 \mathrm{~m}$

Collection(s) - De Vogel 6253; Meijer 10786

POUTERIA

Pouteria celebica Erlee

Sulawesi distribution - South

Altitude - 420-1000 m

Collection(s) - bb 28314; De Vogel 6168; Kjellberg 2325; Van Balgooy 4053

Pouteria maclayana (F. Muell.) Baehni

Sulawesi distribution - North

Collection(s) 一 Lam 3416

Pouteria macrantha (Merr.) Baehni

Vernacular name(s) - Sasangkangan

Sulawesi distribution - North

Altitude $-500 \mathrm{~m}$

Collection(s) - Koorders 18882

Pouteria malaccensis (C.B. Clarke) Baehni

Sulawesi distribution - South

Altitude $-250 \mathrm{~m}$

Collection(s) - Cel II 470, V 209

Pouteria obovata (R. Br.) Baehni

Sulawesi distribution - South-East 
Altitude $-700-900 \mathrm{~m}$

Collection(s) - McDonald \& Ismail Cat 3887

\section{SARCOSPERMA}

Sarcosperma paniculatum (King) Stapf \& King

Sulawesi distribution - Central, South

Altitude $-760 \mathrm{~m}$

Collection(s) — bb 28216; Yoshida 1248

\section{SAXIFRAGACEAE}

\section{DICHROA}

\section{Dichroa fibrifuga Lour.}

Sulawesi distribution - North, Central, South Altitude $-250-2200 \mathrm{~m}$

Collection(s) - Bloembergen 3912, 4004; Bünnemeyer 12051; Meijer 9388, 9803; Mogea 1440; Noerkas 492; Rachmat 468; Ramlanto \& Fanani 464; Van Balgooy 3025, 3291; Yusuf \& Wahyono 74

\section{ITEA}

Itea macrophylla Wall.

Vernacular name(s) - Belante kuhe (Napu) Sulawesi distribution - North, Central, South Altitude - 0-1200 m

Collection(s) - Bünnemeyer 11041, 11230, 11715; De Vogel 5268; Elbert 3227; Meijer 9327; Van Balgooy 3145; Whitmore \& Sidiyasa TCW 3516

\section{POLYOSMA}

Polyosma celebica Schulze-Menz

Vernacular name(s) - Tabee-Tabee

Sulawesi distribution - North, South, SouthEast

Altitude - 1100-3000 m

Collection(s) - bb 8573, 22859, 24200, 24950, 28222; Bünnemeyer 12227, 12228, 12311; Teijsmann HB 14189; Van Steenis 20050

\section{Polyosma integrifolia Blume}

Vernacular name(s) - Tangoero motea
Sulawesi distribution - North, Central, South Altitude - 380-1800 m

Collection(s) - Afriastini \& Rohajawati JJA 2906; bb 1876; Cel III 25; De Vogel 5439, 6333; Meijer 9700; Tantra 1557; Van Balgooy 3413

Polyosma spec.

Sulawesi distribution - South

Altitude - $1600 \mathrm{~m}$

Collection(s) - bb 20783

\section{SCHISANDRACEAE}

\section{KADSURA}

\section{Kadsura lanceolata King}

Sulawesi distribution - Central, South

Lore Lindu National Park \& environs - present Collection(s) - Meijer 9728; Van Balgooy 3536

\section{Kadsura spec.}

Sulawesi distribution - Central, South

Collection(s) - Afriastini 2122; De Vogel 5501

\section{SIMAROUBACEAE}

\section{AILANTHUS}

\section{Ailanthus triphysa (Dennst.) Alston}

Vernacular name(s) - Woeliadolo

Sulawesi distribution - North, South

Altitude - $250 \mathrm{~m}$

Collection(s) - Cel V 177; Koorders 18897

\section{BRUCEA}

\section{Brucea javanica (L.) Merr.}

Vernacular name(s) - Tantaule

Sulawesi distribution - Central, South, SouthEast

Altitude - 0-300 m

Collection(s) - Chin 3428; Elbert 2607, 2807, 2833, 3061, 3301; Eyma 3506, 3507; Prawiroatmodjo \& Maskuri 1073; Prawiroatmodjo \& Soewoko 1626; Teijsmann HB 13898, HB 13907; Widjaja 706 
HARRISONIA

Harrisonia brownii Juss.

Sulawesi distribution - Central, South-East

Collection(s) - Kaudern 445; Kjellberg 47

Harrisonia javanica Blume

Vernacular name(s) - Tambara tedong

Sulawesi distribution - South

Altitude - $200 \mathrm{~m}$

Collection(s) - Cel I 70; Meijer 10961

Harrisonia perforata (Blanco) Merr.

Vernacular name(s) - Moma

Sulawesi distribution - North, Central

Lore Lindu National Park \& environs - present

Altitude - 0-100 m

Collection(s) - bb 17; De Joncheere 1019;

Hennipman 5016; Meijer 9210, 9279, 10166;

Van Balgooy 2978

\section{PICRASMA}

Picrasma javanica Blume

Vernacular name(s) - Wandeboka maeto

Sulawesi distribution - South, Central, North

Lore Lindu National Park \& environs - present

Altitude $-200-650 \mathrm{~m}$

Collection(s) - Cel V 186; De Vogel 5634;

Koorders 18011, 18012, 18013, 19708

QUASSIA

Quassia indica (Gaertn.) Noot.

Sulawesi distribution - North

Collection(s) - Koorders 18014; Meijer 10134

\section{SOLANACEAE}

BROWALLIA

Browallia americana $\mathrm{L}$.

Sulawesi distribution - North

Altitude -800

Collection(s) - Brinkman 44

\section{CAPSICUM}

Capsicum betacea Sendtn.

Vernacular name(s) - Tetang belanda

Sulawesi distribution - North

Altitude $-1050 \mathrm{~m}$

Collection(s) - De Vogel 2495

\section{Capsicum frutescens $\mathrm{L}$.}

Vernacular name(s) - Lada-Lada

Sulawesi distribution - North, Central, South, South-East

Altitude - $900 \mathrm{~m}$

Collection(s) - Bünnemeyer 10558, 11301; De la Savinierre 287; Prawiroatmodjo \& Maskuri 1101

\section{Capsicum minimum Roxb.}

Sulawesi distribution - North, South-East

Altitude - 0-600 m

Collection(s) - Elbert 3267; Kaudern 5

\section{DATURA}

\section{Datura metel L.}

Sulawesi distribution - Central

Lore Lindu National Park \& environs - present

Collection(s) - Meijer 9280

\section{LYCIANTHES}

\section{Lycianthes biflora Bitter}

Sulawesi distribtion - North, Central, South, South-East

Altitude - 0-1200 m

Collection(s) - Alston 15692; Burley, Partomihardjo \& Rachman 3611, 3628; De Vogel 2502; Elbert 2956, 3371; Hennipman 6039; Kaudern 450; Van Balgooy 3556, 3594; Van Vuuren 237

\section{Lycianthes boninensis Bitter}

Sulawesi distribution - Central

Lore Lindu National Park \& environs - present

Altitude - $900 \mathrm{~m}$

Collection(s) - Meijer 10213

Lycianthes manucaling Elmer

Sulawesi distribution - North, South

Altitude $-200 \mathrm{~m}$ 
Collection(s) - Cel V 300

\section{Lycianthes minutipila Bitter}

Sulawesi distribution - Central

Lore Lindu National Park \& environs - present Altitude - $650 \mathrm{~m}$

Collection(s) - Johansson 143

\section{Lycianthes spec.}

Vernacular name(s) - Pokahe [Besoa]

Sulawesi distribution - North, Central

Lore Lindu National Park \& environs - present

Altitude - 350-1050 m

Collection(s) - De Vogel 5600; De Vogel \& Vermeulen 7003

\section{PHYSALIS}

Physalis angulata $\mathrm{L}$.

Sulawesi distribution - North, Central, SouthEast

Altitude - 5-300 m

Collection(s) - Elbert 3174; Kaudern 379; Koorders 18027 .

Physalis minima L.

Vernacular name(s) - Darate

Sulawesi distribution - North, Central

Altitude - 25-750 m

Collection(s) - Bünnemeyer 11779; Kaudern 162; Lam 3032; Reinwardt 926

\section{SOLANUM}

\section{Solanum americanum Mill.}

Vernacular name(s) - Lambate

Sulawesi distribution - North, Central

Lore Lindu National Park \& environs - present

Altitude - 40-1650 m

Collection(s) - Bloembergen 3995; Bünnemeyer 11277; Johansson 567; Kaudern 12; Lam 2836; Van Balgooy 3555

Solanum biflorum Lour.

Sulawesi distribution - North

Altitude $-750 \mathrm{~m}$

Collection(s) - De Vogel 6791

Solanum erianthum D. Don

Sulawesi distribution - North
Collection(s) - De la Savinierre 274

Solanum ferox L. var. involucratum Blume

Sulawesi distribution - North

Altitude - 350-750 m

Collection(s) - Burley, Partomihardjo \& Rachman 3625

Solanum ferox L. var. lasiocarpum (Dunal) Miq.

Vernacular name(s) - Gandi

Sulawesi distribution - North, Central, South, South-East

Altitude - 50-2200 m

Collection(s) - Bünnemeyer 11980; Chin 3559; Johansson 144; McDonald \& Ismail 4122; Meijer 9736; Prawiroatmodjo \& Maskuri 1356

\section{Solanum spec.}

Sulawesi distribution - North

Altitude - 220-1500 m

Collection(s) - De Vogel \& Vermeulen 6451, 7029, 7052, 7271; Forman 237; Milliken 1058

\section{SONNERATIACEAE}

\section{DUABANGA}

\section{Duabanga moluccana Blume}

Vernacular name(s) - Aras; Atesakah; Bolo; Lekotu [Besoa]; Tawo; Warò

Sulawesi distribution - North, Central, South, South-East

Altitude - 6-1000 m

Collection(s) - bb 5558, 6034, 24129, 24506, 28231, 28740, 32446; Burley, Partomihardjo \& Rachman 3613; Cel I 17a, III 7, III 90; De Vogel 5183, 6030; Johansson 101; Koorders 17755, 17757; Lam 2723; Meijer 9157; Noerkas 333, 436; Van Balgooy 3080

\section{SONNERATIA}

\section{Sonneratia alba $\mathrm{Sm}$.}

Vernacular name(s) - Balombo; Béropa Sulawesi distribution - North, South-East Altitude - 0-125 m

Collection(s) - bb 3888, 4186, 4987, 14442, 16709, 17191, 21793; Elbert 2850; Koorders 17766, 17767, 22228; Meijer 9290 
Sonneratia caseolaris (L.) Engl.

Sulawesi distribution - South

Collection(s) - Cel III 85; Noerkas 407

\section{SPHENOSTEMONACEAE}

SPHENOSTEMON

Sphenostemon papuanum (Lauterb.) Steenis

Sulawesi distribution - South

Altitude $-1500 \mathrm{~m}$

Collection(s) - bb 19566, 24083, 24204

\section{STAPHYLEACEAE}

\section{TURPINIA}

Turpinia borneensis (Merr. \& Perry)

B.L. Linden

Sulawesi distribution - North

Altitude $-500 \mathrm{~m}$

Collection(s) - Burley, Partomihardjo \& Rachman 3619

\section{Turpinia montana Kurz}

Sulawesi distribution - Central

Altitude $-1000 \mathrm{~m}$

Collection(s) - Tantra 1533

Turpinia pomifera (Roxb.) DC.

Vernacular name(s) - Lampasia; Tandopae; Tandopoe motoetoe

Sulawesi distribution - North, South, SouthEast

Altitude $-20-500 \mathrm{~m}$

Collection(s) - bb 14328, 21576, 21726; Cel II 238, II 423, II 434; Forman 247; Koorders 18832, 18836; Lam 2855, 3129

\section{Turpinia sphaerocarpa Hassk.}

Sulawesi distribution - North, Central

Lore Lindu National Park \& environs - - present Altitude - 350-1000 m

Collection(s) - Burley, Partomihardjo \& Rachman 3659; De Vogel 5500, 5569; Tantra 1529; Van Balgooy 3513, 3517, 3581

\section{Turpinia spec.}

Sulawesi distribution - North, Central, South Altitude - 40-700 m

Collection(s) - Alston 16260; bb 29478; De Vogel 6038; Forsten 77; Koorders 18835, 18837

\section{STERCULIACEAE}

\section{ABROMA}

Abroma fastuosa R.Br.

Vernacular name(s) - apesa

Sulawesi distribution - North

Altitude - 10-50 m

Collection(s) - Koorders 19468, 19469; Rachmat 241

Abroma spec.

Sulawesi distribution - North

Collection(s) - Alston 16155

FIRMIANA

Firmiana minahassae (Koord.) Kosterm.

Sulawesi distribution - North

Altitude - 50-500 m

Collection(s) - Koorders 18070, 18078

Firmiana spec.

Sulawesi distribution - South-East

Altitude $-150 \mathrm{~m}$

Collection(s) - Coode 6250

\section{HELICTERES}

\section{Helicteres angustifolia L.}

Sulawesi distribution - South, South-East

Altitude - 15-600 m

Collection(s) - bb 5861; Noerkas 330; Van Steenis 10341

Helicteres integerrima Korth.

Sulawesi distribution - South-East

Altitude - 600-700 m

Collection(s) - Elbert 3468 
Helicteres isora L.

Sulawesi distribution - South

Altitude $-50 \mathrm{~m}$

Collection(s) - Bünnemeyer 10610; Rachmat 96

\section{Helicteres spec.}

Sulawesi distribution - South-East

Altitude - 20-250 m

Collection(s) - Prawiroatmodjo \& Soewoko 1694

\section{HERITIERA}

Heritiera arafurensis Kosterm.

Sulawesi distribution - North, Central

Altitude - 10-500 m

Collection(s) - bb 31884, 32484; Koorders 18052, 18053, 18054; Teijsmann HB 12747

Heritiera javanica (Blume) Kosterm.

Sulawesi distribution - North, Central

Altitude - $300 \mathrm{~m}$

Collection(s) -- bb 15713, 28730

Heritiera littoralis Dryand.

Vernacular name(s) - Doengoe; Loengoelo; Roemoe

Sulawesi distribution - North, South, SouthEast

Altitude $-0-75 \mathrm{~m}$

Collection(s) - bb 3682, 5427, 15126, 21124 , 32374; Elbert 2937; Hose 777; Koorders 19453; Meijer 10110; Noerkas 401; Teijsmann HB 12845

\section{Heritiera sylvatica Vidal}

Sulawesi distribution - North, South

Collection(s) - Hose 777; Meijer 10845, 10985

Heritiera trifoliolata (F. Muell.) Kosterm.

Vernacular name(s) - Roemoeng

Sulawesi distribution - North, Central, South, South-East

Altitude - 20-1200 m

Collection(s) - bb 5162, 21701, 22945, 24096, 24116, 24991, 31857; Cel V 362; Koorders 18085, 18086, 18087; Teijsmann HB 5558, HB 13993

\section{Heritiera spec.}

Sulawesi distribution - North

Altitude $-270 \mathrm{~m}$

Collection(s) - Whitmore \& Sidiyasa TCW 3523

\section{KLEINHOVIA}

\section{Kleinhovia hospita L.}

Vernacular name(s) - Andinaka; barah-boang; Gontoge; Paliasa; Paliassa; Palili; Tokoelo

Sulawesi distribution - North, Central, South, South-East

Altitude - 0-950 m

Collection(s) - bb 2247, 4995, 21601; Bünnemeyer 10571, 10737, 11192, 12567; Buwalda 3813; Cel I 40, III 38; Chin 3409, 3472, 3485; De Joncheere 1022; De Vogel 6483; Elbert 3286; Koorders 18055, 18056, 18057; Lam 3188; Meijer 9147; Noerkas 32; Prawiroatmodjo \& Maskuri 1172, 1195; Rachmat 32, 214; Whitmore \& Sidiyasa TCW 3476

\section{MELOCHIA}

\section{Melochia corchorifolia L.}

Vernacular name(s) - Boentoelobatade

Sulawesi distribution - North, South

Altitude $-50 \mathrm{~m}$

Collection(s) - Bünnemeyer 10556; Noerkas 74,78

\section{Melochia pyramidata $\mathrm{L}$.}

Sulawesi distribution - South-East

Collection(s) - Kjellberg 28

Melochia umbellata (Houtt.) Stapf

Vernacular name(s) - bambak'a; Lambiri; Wintonoe

Sulawesi distribution - North, Central, South, South-East

Altitude $-0-1800 \mathrm{~m}$

Collection(s) - bb 5848; Bünnemeyer 11456 , 11646, 11760, 12354; Cel V 164; De Vogel 5913; Elbert 2588, 2745, 2785, 2904, 3029, 3071; Hose 822; Kjellberg 1656; Koorders 18089, 18091, 19718; Lam 2570; Meijer 10713; Rachmat 29; Van Vuuren 265; Whitmore \& Sidiyasa TCW 3368, TCW 3484; Widjaja 622, 689; Yoshida 996 


\section{PTEROCYMBIUM}

Pterocymbium tinctorium (Blume) Kosterm.

Sulawesi distribution - North, Central, South Altitude - 10-200 m

Collection(s) - bb 17043, 18806, 19610, 25542, 28722; Koorders 18060, 18073, 19451, 19465; Meijer 10073a, 10124; Teijsmann HB 11759

Pterocymbium tinctorium (Blume) Kosterm. var. javanicum (R.Br.) Kosterm.

Sulawesi distribution - South

Collection(s) - Cel V 131; Meijer 10754

\section{Pterocymbium spec.}

Sulawesi distribution - North

Altitude $-250 \mathrm{~m}$

Collection(s) - Koorders 19630; Whitmore \& Sidiyasa TCW 3421, TCW 3450

\section{PTEROSPERMUM}

Pterospermum celebicum Miq.

Vernacular name(s) - Bajoe; Banjoro; Kayu bayuk; Roembei monggane; Waluk'a; Wolo

Sulawesi distribution - North, Central, South, South-East

Altitude $-0-500 \mathrm{~m}$

Collection(s) - bb 4191, 7506, 7527, 14212, 17643, 19436, 20326, 21346, 28276, 28719 . 33090, 34349; Cel I 27, V 213, V 327; De Vogel 2588; Forsten 323; Koorders 18061, 18066, 18067, 18068, 19462, 19475, 19840; Kostermans \& Soegeng Reksodihardjo 1; Lam 3009, 3103; Meijer 10872; Rachmat 776; Teijsmann HB 12441; Van Balgooy 4881; Whitmore \& Sidiyasa TCW 3536; Wind 3504

\section{Pterospermum diversifolium Blume}

Vernacular name(s) - Laua; Woloh bela Sulawesi distribution - North, Central, South Altitude - 50-60 m

Collection(s) - bb 24113, 31494; Koorders 18062, 18063; Meijer 10053; Noerkas 163

Pterospermum javanicum Jungh.

Sulawesi distribution - Central Lore Lindu National Park \& environs — present
Altitude $-800 \mathrm{~m}$

Collection(s) - Meijer 9299

Pterospermum macrocarpum Hochr.

Sulawesi distribution - South-East

Altitude $-20-250 \mathrm{~m}$

Collection(s) - Prawiroatmodjo \& Soewoko 1620

Pterospermum subpeltatum C.B. Rob.

Sulawesi distribution - South

Collection(s) - Teijsmann HB 11972

Pterospermum subsessile Miq.

Sulawesi distribution - North

Collection(s) - De Vriese \& Teijsmann 33

\section{Pterospermum spec.}

Vernacular name(s) - Wayu [Kolaki]

Sulawesi distribution - Central, South-East

Lore Lindu National Park \& environs - present

Altitude $-200 \mathrm{~m}$

Collection(s) - De Vogel 5032, 6062

\section{PTERYGOTA}

Pterygota horsfieldii (R.Br.) Kosterm.

Sulawesi distribution - North

Altitude $-80 \mathrm{~m}$

Collection(s) - bb 17633

\section{STERCULIA}

Sterculia comosa Wall.

Vernacular name(s) - Lawea

Sulawesi distribution - North

Altitude $-0-50 \mathrm{~m}$

Collection(s) - bb 17208; Hochreutiner 8; Koorders 18079, 18083, 18084; Lam 3362; Meijer 10073, 10105

\section{Sterculia foetida L.}

Sulawesi distribution - North, Central, SouthEast

Lore Lindu National Park \& environs - present Altitude - 30-100 m

Collection(s) - bb 21588; De Vogel 5018; Koorders 17804, 17806, 19481; Meijer 9163 
Sterculia insularis R.Br.

Vernacular name(s) - Bingiladu; Kaloempang tjenda; Kambaho; Koelinei poete

Sulawesi distribution - North, South, SouthEast

Altitude - 0-430 m

Collection(s) - bb 28280; Cel I 82, III 44; Church \& Ismail 26; Elbert 2722, 3320, 3433; Lam 2624, 2905; Meijer 10804; Noerkas 342; Rachmat 725; Van Hulstijn \& Atjeh 323

Sterculia kostermansiana Tantra

Sulawesi distribution - South

Collection(s) - Cel 170

Sterculia Iongifolia Tantra

Sulawesi distribution - Central

Lore Lindu National Park \& environs - present Altitude - $200 \mathrm{~m}$

Collection(s) - Van Balgooy 2998

Sterculia macrophylla Vent.

Vernacular name(s) - Banjilan; Mapangi; Moropangi; Sobo

Sulawesi distribution - North, Central, South, South-East

Altitude - 1-50 m

Collection(s) - bb 5872, 29477; Cel III 78; Koorders 15808, 18071, 18072, 18074, 18080; Meijer 10789, 10993; Ramlanto 226

Sterculia oblongata R.Br.

Vernacular name(s) - Kaloempe; Mawaweka Sulawesi distribution - North, South-East

Altitude - 15-750 m

Collection(s) - bb 21361; Koorders 18075, 18076, 18077, 18082, 19450, 19454, 19474; Rachmat 775

\section{Sterculia rubiginosa Vent.}

Sulawesi distribution - North

Collection(s) - Koorders 19470

Sterculia stipulata Korth.

Sulawesi distribution - Central

Lore Lindu National Park \& environs - present Altitude $-200-800 \mathrm{~m}$

Collection(s) - Meijer 9371; Van Balgooy 3078

\section{Sterculia urceolata Sm.}

Sulawesi distribution - North, South

Altitude $-3-600 \mathrm{~m}$

Collection(s) - bb 31895; Burley, Partomihardjo \& Rachman 3781; Cel II 235; Ramlanto 220

\section{Sterculia spec.}

Sulawesi distribution - North, Central, South, South-East

Altitude - 20-400

Collection(s) - Alston 16073, 16175, 16182; Chin 3553, 3580; De Vogel 5727; Hennipman 5770; Prance 30102; Prawiroatmodjo \& Soewoko 1731; Ramlanto \& Fanani 677; Whitmore \& Sidiyasa TCW 3547

\section{STYRACACEAE}

\section{BRUINSMIA}

Bruinsmia styracoides Boerl. \& Koord.

Sulawesi distribution - North

Altitude - 400-800 m

Collection(s) - bb 12659; Koorders 18092

\section{STYRAX}

\section{Styrax spec.}

Sulawesi distribution - South-East

Collection(s) - Coode 6147

\section{SYMPLOCACEAE}

\section{SYMPLOCOS}

Symplocos maliliensis Noot.

Sulawesi distribution - South

Altitude $-0-200 \mathrm{~m}$

Collection(s) - bb 353, 374

Symplocos odoratissima Choisy \& Zoll. var. odoratissima

Sulawesi distribution - North, South-East

Collection(s) - bb 12660, 20761; Forman 265; Milliken 933 
Symplocos pendula Wight var. hirtistylis (Clarke) Noot.

Sulawesi distribution - Central

Lore Lindu National Park \& environs - present Altitude $-2000 \mathrm{~m}$

Collection(s) - Van Balgooy 3258

Symplocos spec.

Vernacular name(s) - Belante [Besoa]

Sulawesi distribution - Central

Lore Lindu National Park \& environs - present Altitude - $1050 \mathrm{~m}$

Collection(s) - De Vogel 5614

\section{TAXACEAE}

\section{TAXUS}

Taxus sumatrana (Miq.) de Laub.

Sulawesi distribution - Central, South

Altitude - 1200-2000 m

Collection(s) - bb 19577, 20231, 20887, 24961, 33075; Eyma 1074; Teijsmann HB 14190

\section{THEACEAE}

\section{ADINANDRA}

Adinandra brefeldii Koord.

Sulawesi distribution - Central, North

Altitude - 300-900 m

Collection(s) - bb 20271; Koorders 18927, $18963,18965,18967,18968$

\section{Adinandra celebica Koord.}

Vernacular name(s) - Ilasilala; Tangoeroe

Sulawesi distribution - North, Central, South, South-East

Altitude - 15-1200 m

Collection(s) - Amir 80, 177; bb 5860, 21788, 23219; Cel II 362, IV 88; Koorders 18096, 18098; Meijer 9725A; Ramlanto 184

Adinandra eymae Kobuski

Sulawesi distribution - South

Altitude - 2600-3000 m

Collection(s) - Eyma 588

\section{Adinandra masambensis Kobuski}

Sulawesi distribution - South

Altitude - $1600 \mathrm{~m}$

Collection(s) - bb 26298

\section{Adinandra steenisii Kobuski}

Sulawesi distribution - Central

Altitude $-2800 \mathrm{~m}$

Collection(s) - Van Steenis 10303

\section{Adinandra spec.}

Sulawesi distribution - North, Central, South, South-East

Altitude - 350-2000 m

Collection(s) - Coode 6180, 6187; De Vogel 5479, 5680, 5681, 5716, 6069, 6233; Eyma 1681, 3349; Tantra 1593; Van Balgooy 3224, $3268,3402,3661,3978$; Whitmore \& Sidiyasa TCW 3506; Yoshida 1447

\section{CAMELLIA}

Camellia lanceolata (Blume) Seem.

Sulawesi distribution - North, Central, South, South-East

Altitude - 150-1900 m

Collection(s) - bb 18081, 24132; Bünnemeyer 11130, 11321, 11475, 12145; Burley, Partomihardjo \& Rachman 3661, 3839; Koorders $18100,18902,18966,19286,19287$; Meijer $9494,9615,11197$; Prawiroatmodjo \& Maskuri 1139, 1457, 1589; Teijsmann HB 13982, HB 14193; Van Balgooy 3467, 4058

Camellia spec.

Sulawesi distribution - South, South-East

Altitude - 1250-1900 m

Collection(s) - Coode 6155, 6159; Meijer 10791; Sands 470

\section{EURYA}

Eurya acuminata DC.

Sulawesi distribution - North, South

Lore Lindu National Park \& environs - present Altitude - 1500-1700 m

Collection(s) - Koorders 18903; Meijer 9848

Eurya celebica Reinw. ex Korth.

Sulawesi distribution - North, South

Altitude - 0-2640 m 
Collection(s) - Koorders 18904; Reinwardt 1569; Sands 576

Eurya chinensis R.Br. subsp. flava (Melch.) de Wit

Sulawesi distribution — North, South

Lore Lindu National Park \& environs - present

Altitude - 1600-3000 m

Collection(s) - Bloembergen 3905; Eyma 735, 793

Eurya japonica Thunb.

Sulawesi distribution - North

Lore Lindu National Park \& environs - present Altitude - 1650-2000 m

Collection(s) - bb 18099; Bloembergen 4007; Koorders 18907

Eurya obovata (Blume) Korth. var. reticulata (Korth.) de Wit

Sulawesi distribution - South

Altitude - 2500-2750 m

Collection(s) - Bünnemeyer 11938, 12180

Eurya oxysepala Diels

Sulawesi distribution - South

Altitude $-1400 \mathrm{~m}$

Collection(s) - Mamahit 17

\section{Eurya spec.}

Sulawesi distribution - North, Central, South Altitude $-3-2000 \mathrm{~m}$

Collection(s) - Alston 15856; De Vogel 5351, 5678; Hennipman 5758; Kofman 198; Meijer 11505a; Sands 627; Van Balgooy 3264, 3397

\section{GORDONIA}

Gordonia amboinensis (Miq.) Merr.

Vernacular name(s) - Solompe

Sulawesi distribution - North, Central, South

Altitude - 150-1300 m

Collection(s) - Cel II 481; Koorders 18910 , 18912, 18913, 18914; Meijer 9487; Van Balgooy $3414,3524,3896$

\section{Gordonia integerrima (Miq.) H. Keng}

Sulawesi distribution - North

Altitude $-1800 \mathrm{~m}$

Collection(s) — bb 18095; Koorders 18099

\section{Gordonia spec.}

Sulawesi distribution - Central, South

Lore Lindu National Park \& environs - present Altitude - 40-2000 m

Collection(s) - bb 23942, 24205; De Vogel 5441, 6223; Tantra 1590; Yusuf \& Wahyono 53

\section{PYRENARIA}

\section{Pyrenaria wrayi King}

Sulawesi distribution - North

Collection(s) - Koorders 18916

\section{SCHIMA}

Schima wallichii (DC.) Korth.

Sulawesi distribution - South

Collection(s) — Meijer 10747

\section{TERNSTROEMIA}

Ternstroemia elongata (Korth.) Koord.

Vernacular name(s) - Sakoeang

Sulawesi distribution - South

Altitude $-1650 \mathrm{~m}$

Collection(s) - bb 8572

Ternstroemia japonica Thunb.

Sulawesi distribution - South-East

Altitude - 600-700 m

Collection(s) - Elbert 3473

\section{Ternstroemia penangiana Choisy}

Vernacular name(s) - Kay atellu

Sulawesi distribution - North

Collection(s) - Riedel 5816

Ternstroemia toquian (Blanco) Fern.-Vill.

Sulawesi distribution - North, South

Altitude $-500 \mathrm{~m}$

Collection(s) - Koorders 18911; Yusuf \& Wahyono 18

Ternstroemia urdanetensis (Elmer) Kobuski var. crassifolia Kobuski

Sulawesi distribution - South

Altitude $-2800 \mathrm{~m}$

Collection(s) - Eyma 613 


\section{Ternstroemia spec.}

Sulawesi distribution - North, Central, South Lore Lindu National Park \& environs - present Altitude - 300-2350 m

Collection(s) - bb 24082, 24191, 24196, 26295, 28221, 28261; Darnaedi 1885; De Vogel 5359, 6082, 6158, 6309, 6319, 6322, 6330; Eyma 1461, 3631; Kjellberg 2167; Koorders 19622; Noerkas 479; Ramlanto 208; Van Balgooy 3283, 3354, 3377, 3773, 4048; Whitmore \& Sidiyasa TCW 3541

\section{THYMELAEACEAE}

\section{GONYSTYLUS}

Gonystylus macrophyllus (Miq.) Airy Shaw

Sulawesi distribution - North, South

Altitude - 5-400 m

Collection(s) - bb 31508; Burley, Partomihardjo \& Rachman 3632; De Vogel 6197; Lam 2856, 2906; Meijer 10854; Teijsmann HB 12087

\section{GYRINOPS}

Gyrinops decipiens Ding Hou

Sulawesi distribution - South

Altitude - 100-420 m

Collection(s) - De Vogel 6169; Kjellberg 889

Gyrinops moluccana (Miq.) Baill.

Sulawesi distribution - South

Altitude - $430 \mathrm{~m}$

Collection(s) - De Vogel 6245

\section{PHALERIA}

\section{Phaleria capitata Jack}

Vernacular name(s) - La'awan'a; Tomalojo Sulawesi distribution - North, Central

Altitude - 0-750 m

Collection(s) - Burley, Partomihardjo \& Rachman 3707, 3716, 3825, 3845, 3971, 4004; De Vogel \& Vermeulen 6614, 6716, 7055, 7058; Forsten 360; Kaudern 101; Koorders 18678, 18969, 18970, 18971, 18972; Lam 2702; Posthumus 2541; Ramlanto \& Fanani 614; Reinwardt 1511; Riedel 23, 5910, HB 5870; Whitmore \& Sidiyasa TCW 3535
Phaleria coccinea (Gaudich.) F. Muell.

Sulawesi distribution - Central

Lore Lindu National Park \& environs — present Altitude $-725 \mathrm{~m}$

Collection(s) - Johansson 115

Phaleria octandra (L.) Baill.

Sulawesi distribution - South

Collection(s) - Meijer 10729, 11431

Phaleria spec.

Sulawesi distribution - North, South

Altitude - 220-1100

Collection(s) - De Vogel \& Vermeulen 6505; Hennipman 5997

\section{WIKSTROEMIA}

Wikstroemia androsaemifolia Decne.

Sulawesi distribution - North, South, SouthEast

Altitude - 400-1600 m

Collection(s) - Alston 16084; Bloembergen 3994; Coode 6150; Meijer 11102A; Van Balgooy 4043

Wikstroemia indica (L.) C.A. Mey.

Sulawesi distribution - South-East

Altitude $-1500 \mathrm{~m}$

Collection(s) - McDonald \& Ismail Cat 4066

Wikstroemia tenuiramis Miq.

Sulawesi distribution - North, Central

Altitude - 150-200 m

Collection(s) - Meijer 10238; Van Balgooy 2967

\section{Wikstroemia spec.}

Sulawesi distribution - Central

Lore Lindu National Park \& environs - present Altitude - $1900 \mathrm{~m}$

Collection(s) 一 De Vogel 5155

\section{TILIACEAE}

BROWNLOWIA

Brownlowia lanceolata Benth.

Sulawesi distribution - North 
Collection(s) — Lam 2491

\section{COLONA}

\section{Colona celebica Burret}

Sulawesi distribution - North

Collection(s) — Riedel HB 5684

Colona scabra (Sm.) Burret

Vernacular name(s) - Anit'a

Sulawesi distribution - North, Central, SouthEast

Altitude - 0-150 m

Collection(s) - bb 4996, 20758, 28724; Elbert 2680; Koorders 18973; Lam 3044, 3076; Meijer 10144

\section{Colona serratifolia Cav.}

Vernacular name(s) - Konito; Taboekang

Sulawesi distribution - North, South

Altitude $-0-200 \mathrm{~m}$

Collection(s) - bb 7503; Cel I 5; Koorders 18974, 18975, 18976; Noerkas 111

\section{Colona spec.}

Vernacular name(s) - Bolang djese; Samoeking Sulawesi distribution - Central, South

Altitude - 3-50 m

Collection(s) - bb 18799; Bünnemeyer 10646; Noerkas 246, 393; Rachmat 738; Ramlanto 215

\section{CORCHORUS}

\section{Corchorus aestuans L.}

Vernacular name(s) - loempansit'a

Sulawesi distribution - North, South

Altitude $-5 \mathrm{~m}$

Collection(s) - Lam 3344; Noerkas 53

Corchorus spec.

Sulawesi distribution - South

Collection(s) - Chin 3388

\section{GREWIA}

Grewia acuminata Juss.

Sulawesi distribution - Central, South

Collection(s) - Coode 5910; Meijer 10664
Grewia columnaris Sm.

Sulawesi distribution - North

Altitude $-50 \mathrm{~m}$

Collection(s) - Koorders 19015

Grewia glabra Blume

Vernacular name(s) - Lenoeroeng; oudilotahi [moronene]

Sulawesi distribution - North, Central, South, South-East

Altitude - 50-700 m

Collection(s) - Bünnemeyer 10703, 11367, 11791; Cel I 32; Kjellberg 1281; Koorders 19012, 19013, 19017, 19073; Teijsmann HB 12635, HB 13646, HB 18037; Widjaja 671

\section{Grewia multiflora Juss.}

Sulawesi distribution - North, Central, South Lore Lindu National Park \& environs - present Altitude $-700 \mathrm{~m}$

Collection(s) - Koorders 19020; Meijer 9145, 10191, 10945

\section{Grewia scabrida Wall.}

Sulawesi distribution - South

Collection(s) - Teijsmann HB 12326

\section{Grewia umbellata Roxb.}

Sulawesi distribution - North, South-East

Altitude - 0-500 m

Collection(s) - Kjellberg 364; Koorders 19014 , $19016,19018,19019$

\section{Grewia spec.}

Vernacular name(s) - bisuka [Besoa]; Bitunu [Besoa]; Bunut; Kapoe marum poetik; woke [Besoa]

Sulawesi distribution - North, Central, South, South-East

Lore Lindu National Park \& environs - present Altitude - 0-1050 m

Collection(s) - Alston 15901, 16081, 16180; bb 16990, 17236, 21767; Bloembergen 4103; Buwalda 3794; Chin 3655; Coode 5899; De Vogel 5035, 5053, 5162, 5541, 5615; De Vogel \& Vermeulen 6731; De Vriese 429; De Vriese \& Teijsmann 54; Elbert 2516, 2560, 2637, 2868; Keßler et al. PK 3152; Tantra 1548; Van Balgooy 2992 
MICROCOS

\section{Microcos paniculata $\mathrm{L}$.}

Vernacular name(s) - Boenoe

Sulawesi distribution - South

Altitude $-200 \mathrm{~m}$

Collection(s) - Cel I 42

Microcos stylocarpa (Warb.) Burret

Sulawesi distribution - North

Altitude $-450 \mathrm{~m}$

Collection(s) - Burley, Partomihardjo \& Rachman 3597

\section{Microcos spec.}

Sulawesi distribution - North, South, SouthEast

Altitude $-20-750 \mathrm{~m}$

Collection(s) - bb 69; Chin 3496; De Vogel 6774; Prawiroatmodjo \& Maskuri 1357; Prawiroatmodjo \& Soewoko 1963

\section{TRICHOSPERMUM}

\section{Trichospermum kjellbergii Burret}

Vernacular name(s) - woenoe

Sulawesi distribution - South

Altitude - 0-400 m

Collection(s) - Cel II 218, III 6; Darnaedi 1992; De Vogel 5954, 5957; Meijer 11313; Van Balgooy 3782, 3898, 3999

Trichospermum lanigerum (Blume) Merr.

Sulawesi distribution - South

Collection(s) - Noerkas 432

Trichospermum pleiostigma (F. Muell.) Kosterm.

Sulawesi distribution - Central, South-East Altitude $-10-630 \mathrm{~m}$

Collection(s) - bb 21616, 25003

Trichospermum talaudensis Kosterm.

Vernacular name(s) - siapae

Sulawesi distribution - North

Altitude $-40 \mathrm{~m}$

Collection(s) — Lam 2983

\section{TRIUMFETTA}

Triumfetta annua $L$.

Sulawesi distribution — South

Collection(s) - Meijer 10685

\section{Triumfetta bartramia L.}

Vernacular name(s) - Pojo-pojo; tangkapakapa

Sulawesi distribution - North, Central, South, South-East

Altitude $-0-950 \mathrm{~m}$

Collection(s) - Bünnemeyer 10870, 10992, 12546; Elbert 2682, 2871; Koorders 17838, 19022; Noerkas 272; Rachmat 75; Widjaja 614

Triumfetta cana Blume

Sulawesi distribution - Central

Altitude - $950 \mathrm{~m}$

Collection(s) -- Bünnemeyer 10969, 11089

Triumfetta pilosa Roth.

Sulawesi distribution - North

Altitude $-800 \mathrm{~m}$

Collection(s) - Koorders 19025

Triumfetta procumbens G. Forst.

Sulawesi distribution - North

Altitude $-1 \mathrm{~m}$

Collection(s) - Lam 3366, 3450

Triumfetta suffruticosa Blume

Sulawesi distribution - North

Collection(s) - Noerkas 494

Triumfetta tomentosa Bojer

Sulawesi distribution - South

Collection(s) - Rachmat 222

Triumfetta spec.

Sulawesi distribution - North, South

Collection(s) - Yoshida 1121 


\section{TURNERACEAE}

TURNERA

\section{Turnera ulmifolia L.}

Sulawesi distribution - South

Collection(s) - Hidayat AH 294

\section{ULMACEAE}

\section{APHANANTHE}

Aphananthe cuspidata Planch.

Sulawesi distribution - North, South

Altitude $-500 \mathrm{~m}$

Collection(s) - bb 17163; Koorders 18550; Teijsmann HB 13967

\section{CELTIS}

\section{Celtis philippensis Blanco}

Vernacular name(s) - Kanuna [Uma]; Kaoloeloe; Woloeloe Morokotoeo

Sulawesi distribution - Central, South, SouthEast

Lore Lindu National Park \& environs - present Altitude - 0-650 m

Collection(s) - Afriastini 1990; bb 11427 , 17058, 17507, 17647, 21369, 21374, 22982, 24965, 29467; Cel V 160; De Vogel 5622, 5674, 5762; Elbert 3363; Hennipman 5765; Koorders 16379, 19072, 19075; Meijer 9223, 10859; Noerkas 458; Van Balgooy 2973

Celtis rigescens (Miq.) Planch.

Sulawesi distribution - North, Central, South Altitude - 500-600 m

Collection(s) - bb 17001; Bloembergen 4231; Koorders 19068, 19079

\section{Celtis spec.}

Sulawesi distribution - North

Altitude - $250 \mathrm{~m}$

Collection(s) - Whitmore \& Sidiyasa TCW 3399

\section{GIRONNIERA}

Gironniera subaequalis Planch.

Vernacular name(s) - Awoe; Ilaoe sala; Ponto mentega

Sulawesi distribution - Central, South, SouthEast

Altitude - 20-645 m

Collection(s) - bb 1825, 1844, 1845, 1847, $1848,1900,1912,1922,1924,1928,1929$, $2359,11418,23255,24496,26014,26283$, 31816, 31821; Cel II 412, II 432; Darnaedi 2275; De Vogel 6085, 6308; Eyma 4088; Meijer 11235; Prawiroatmodjo \& Soewoko 1708; Ramlanto 204; Van Balgooy 3789, 3939

\section{Gironniera spec.}

Sulawesi distribution - South, South-East

Altitude - 20-450 m

Collection(s) - De Vogel 5972; Prawiroatmodjo \& Soewoko 1743

\section{PARASPONIA}

Parasponia rugosa Blume

Vernacular name(s) — Lahendong

Sulawesi distribution - North

Altitude $-0-1300 \mathrm{~m}$

Collection(s) - Koorders 19422, 19423

TREMA

Trema angustifolia Planch.

Sulawesi distribution - South

Collection(s) - Meijer 11512; Rachmat 392

Trema cannabina Lour.

Vernacular name(s) - Mandalirung'a

Sulawesi distribution - North, Central, South, South-East

Altitude $-0-50 \mathrm{~m}$

Collection(s) - bb 5606; Elbert 2576, 2938; Lam 3331; Rachmat 221; Teijsmann HB 12867, HB 13884, HB 13908

Trema orientalis (L.) Blume

Vernacular name(s) - Mawa; Sponia-Mawa; Wulaja [Besoa]

Sulawesi distribution - North, Central, South, South-East 
Altitude - 15-1425 m

Collection(s) - bb 5157, 22077, 28215; Cel V 138; De Vogel 2577, 5161, 5690; De Vogel \& Vermeulen 6596, 6663; Koorders 19426; Meijer 10142, 11020, 11080; Teijsmann HB 13621; Whitmore \& Sidiyasa TCW 3483; Widjaja 690

Trema tomentosa (Roxb.) Hara

Sulawesi distribution - North, Central, South, South-East

Altitude - 0-1150 m

Collection(s) - bb 23595; Cel V 288; De Vogel 5915; Elbert 3345; Eyma 3358, 3359; Grimes 1031; Kaudern 290; Teijsmann HB 12667

Trema spec.

Sulawesi distribution - North, Central, South Altitude - 220-650 m

Collection(s) - De Vogel \& Vermeulen 6459; Johansson 411; Van Balgooy 4050

\section{URTICACEAE}

\section{BOEHMERIA}

\section{Boehmeria spec.}

Sulawesi distribution - North, South Altitude - 220-1000 m

Collection(s) - Alston 15662; Chin 3447, 3629, 3661; De Vogel \& Vermeulen 6920, 6922; Whitmore \& Sidiyasa TCW 3372

\section{CHAMABAINIA}

Chamabainia cuspidata Wight

Sulawesi distribution - South

Altitude $-2900 \mathrm{~m}$

Collection(s) — Monod de Froideville 247

\section{CYPHOLOPHUS}

Cypholophus lutescens (Blume) Wedd.

Sulawesi distribution - North, South

Altitude - 2000-2900 m

Collection(s) - Bünnemeyer 12054; Eyma 731, 3926

\section{Cypholophus macrocephalus Wedd.}

Vernacular name(s) - sosop

Sulawesi distribution - North, South-East

Altitude - 40-300 m

Collection(s) - Elbert 3189; Lam 2765

\section{Cypholophus spec.}

Vernacular name(s) - Hohora

Sulawesi distribution - North, Central, South

Altitude - 250-650 m

Collection(s) - De Vogel \& Vermeulen 6879; Eyma 3867, 4180; Johansson 97; Whitmore \& Sidiyasa TCW 3446

\section{DEBREGEASIA}

Debregeasia velutina Gaudich.

Vernacular name(s) - Kajoe-Koang Sulawesi distribution - Central, South

Lore Lindu National Park \& environs - present Altitude - 650-1450 m

Collection(s) - Bünnemeyer 11598; Johansson 275; Teijsmann HB 14189

\section{Debregeasia spec.}

Vernacular name(s) - Ruwiran / Kakeni [Tombulu / Tontembuan]

Sulawesi distribution - North

Collection(s) - Alston 16162

\section{DENDROCNIDE}

Dendrocnide crassifolia (C.B. Rob.) Chew

Vernacular name(s) - Sarai

Sulawesi distribution - North

Altitude $-20 \mathrm{~m}$

Collection(s) - Lam 3007

\section{Dendrocnide kjellbergii Chew}

Vernacular name(s) - Glata; Latang

Sulawesi distribution - Central, South

Altitude - 50-950 m

Collection(s) - Bünnemeyer 11108; Buwalda 3809; Eyma 3536, 3734

Dendrocnide longifolia (Hemsl.) Chew

Sulawesi distribution - South

Collection(s) - Meijer 10893 
Dendrocnide microstigma (Gaudich. ex Wedd.) Chew

Vernacular name(s) - Lalasang; Makarrenang; Pahawong/Kulok/Boehn; Tahi

Sulawesi distribution - Central, South, SouthEast

Lore Lindu National Park \& environs - present Altitude $-25-800 \mathrm{~m}$

Collection(s) - bb 21708; Cel I 57, V 120; Church \& Ismail 5; De Vriese 40, 41; Elbert 2768; Forsten 9; Meijer 9305, 10759; Teijsmann HB 11711, HB 13794

\section{Dendrocnide oblanceolata (Merr.) Chew}

Sulawesi distribution - Central

Lore Lindu National Park \& environs — present Altitude - $1000 \mathrm{~m}$

Collection(s) — De Vogel 5525

Dendrocnide stimulans (L.f.) Chew

Sulawesi distribution - North, Central

Lore Lindu National Park \& environs - present

Altitude - 50-1000 m

Collection(s) - De Vogel 5180; Koorders 19391,19393

\section{Dendrocnide spec.}

Vernacular name(s) - Jilatah/Lelati [Uma]; Lana/Pawang/Daun sos [Tontembuan/ Tolour]

Sulawesi distribution - North, Central, South Altitude $-600-1100 \mathrm{~m}$

Collection(s) - Alston 16259; Chin 3483; De Joncheere 1214; Hennipman 6112; Johansson 153; Tantra 1538; Van Balgooy 3088, 3532

\section{GIRARDINIA}

Girardinia diversifolia (Link) Friis

Vernacular name(s) - Gamassa

Sulawesi distribution - South

Altitude - $2060 \mathrm{~m}$

Collection(s) - Bünnemeyer 12015

\section{LECANTHUS}

Lecanthus peduncularis (Royle) Wedd.

Sulawesi distribution - South

\author{
Altitude - 1200-2400 m \\ Collection(s) - Bünnemeyer 11853, 12041; \\ Eyma 1500; J.B.M. Smith 621
}

\section{LEUCOSYKE}

\section{Leucosyke candidissima Wedd.}

Sulawesi distribution - South-East

Altitude $-50 \mathrm{~m}$

Collection(s) - Prawiroatmodjo \& Soewoko 1325

Leucosyke capitellata (Poir.) Wedd.

Vernacular name(s) - Ruwiran [Langowan] Sulawesi distribution - North, Central, South Altitude - 60-1200 m

Collection(s) - Cel V 262; Chin 3387; Coode 5822; De Vogel 2494; De Vogel \& Vermeulen 6504; Forman 182, 400; Hennipman 6071; Meijer 9242

\section{Leucosyke spec.}

Sulawesi distribution - North, Central, South, South-East

Altitude - 10-1000 m

Collection(s) - Bloembergen 4094; Buwalda 3803; Coode 5986; De Vogel 5951, 6050; Elbert 3152, 3185, 3462; Eyma 3527, 3538 , 3808, 4161; Ramlanto 219; Ramlanto \& Fanani 469; Van Balgooy 3783; Whitmore \& Sidiyasa TCW 3370; Yoshida 1199

\section{NOTHOCNIDE}

Nothocnide mollissima (Blume) Chew

Sulawesi distribution - Central

Lore Lindu National Park \& environs - present Altitude $-1000 \mathrm{~m}$

Collection(s) - Hennipman 5668; De Vogel $5066,5250,5255$

Nothocnide repanda (Blume) Blume

Sulawesi distribution - North, Central Altitude - 100-1000 m

Collection(s) - Burley, Partomihardjo \& Rachman 3517; De Vogel 5530; De Vogel \& Vermeulen 6750A; Koorders 19064; Van Balgooy 3525 


\section{OREOCNIDE}

Oreocnide semicrenata Miq.

Sulawesi distribution - South-East

Altitude - 0-320 m

Collection(s) - Elbert 2651, 2752

Oreocnide spec.

Sulawesi distribution - North

Altitude $-750 \mathrm{~m}$

Collection(s) - Kaudern 167, 530

PILEA

Pilea angulata Blume

Sulawesi distribution - South

Altitude $-280 \mathrm{~m}$

Collection(s) - Bünnemeyer 10873

Pilea johniana Stapf

Sulawesi distribution - South

Altitude - 2860-3440 m

Collection(s) - Eyma 861, 1393; J.B.M. Smith 675

Pilea smilacifolia (Wall.) Wedd.

Sulawesi distribution - South-East

Collection(s) - Kaudern 305

Pilea venulosa Blume

Sulawesi distribution - North, South

Collection(s) - Noerkas 348

Pilea wightii Wedd.

Sulawesi distribution - South

Altitude $-1500-2500 \mathrm{~m}$

Collection(s) - Bünnemeyer 11577, 11836, 11915

\section{Pilea spec.}

Sulawesi distribution - North, Central, South, South-East

Altitude $-20-3000 \mathrm{~m}$

Collection(s) - Bloembergen 3978; Chin 3501, 3638; Coode 6004, 6240; De Vogel 5451; Elbert 2711; Eyma 721, 724, 1054, 1403, 1453, 3846, 4171; Johansson 31; Meijer 9128 , 9769, 9861, 9909; Rachmat 429; Soejatmi 264; Van Balgooy 3499, 3905; Yoshida 1004
PIPTURUS

\section{Pipturus spec.}

Sulawesi distribution - Central

Lore Lindu National Park \& environs - present Altitude - $1025 \mathrm{~m}$

Collection(s) - Keßler et al. PK 3148

POUZOLZIA

\section{Pouzolzia spec.}

Sulawesi distribution - South

Collection(s) - Buwalda 3753; De Vogel \& Vermeulen 6822; Eyma 1213; Johansson 526; Koorders 19384; Van Balgooy 3110

\section{PROCRIS}

Procris reticulatovenosa (Hallier f.) H. Schroet.

Sulawesi distribution - South

Collection(s) - Teijsmann HB 13980

\section{VILLEBRUNEA}

Villebrunea rubescens (Blume) Miq.

Vernacular name(s) - ambira; boerala'a; Kaloempenga; Lalang usan [Tonsea]; Padila

Sulawesi distribution - Central, South

Lore Lindu National Park \& environs - present Altitude - 10-1275 m

Collection(s) - Alston 16043; bb 2240; Bünnemeyer 11489, 11680; Cel I 71, V 248; Coode 6097; De Vogel 2491, 2545, 2646, 5196, 5331; De Vogel \& Vermeulen 6882; Elbert 3036; Forman 193, 332; Kaudern 167, 530; Koorders 19427, 19733; Lam 2412, 2720 , 2810; Meijer 10751, 10904; Rachmat 836; Teijsmann HB 11591, HB 12655; Whitmore \& Sidiyasa TCW 3381

\section{Villebrunea spec.}

Sulawesi distribution - Central, South

Altitude - 50-1200 m

Collection(s) - Buwalda 3724; Coode 5808; Johansson 314; Ramlanto 242 


\section{VERBENACEAE}

\section{CALLICARPA}

Callicarpa buchanani (Roxb.) Walp.

Sulawesi distribution - South-East

Altitude - 0-60 m

Collection(s) - Elbert 2545

Callicarpa candicans (Burm.f.) Hochr.

Vernacular name(s) - roidi

Sulawesi distribution - North, Central, South, South-East

Altitude $-0-750 \mathrm{~m}$

Collection(s) - bb 12; Burley, Partomihardjo \& Rachman 3974; Elbert 2538; Kjellberg 14; Koorders 19491, 19494; Lam 3372; Meijer 10153; Noerkas 422; Robinson 2465

\section{Callicarpa formosana Rolfe}

Sulawesi distribution - South

Altitude - $350 \mathrm{~m}$

Collection(s) - Yusuf \& Wahyono 36

\section{Callicarpa longifolia Lam.}

Sulawesi distribution - North, Central, South, South-East

Altitude - 50-1490 m

Collection(s) - Bünnemeyer 10643; 11014, 11707, 12580; Kaudern 313, 424; Kjellberg 725; Koorders 19489; Meijer 9708; Rachmat 588; Sands 474

\section{Callicarpa longifolia Lam. forma floccosa} Schau

Sulawesi distribution - Central, South East Altitude - 50-100 m

Collection(s) - Prawiroatmodjo \& Maskuri 1291; Ramlanto \& Fanani 751

\section{Callicarpa rubella Lindl.}

Vernacular name(s) - Bali-bali angin Sulawesi distribution - South, South-East Altitude $-0-100 \mathrm{~m}$

Collection(s) - Elbert 2814; Rachmat 206

\section{Callicarpa spec.}

Vernacular name(s) — Gadu Lenanju [Kolawi] Sulawesi distribution - North, Central, South, South-East
Lore Lindu National Park \& environs — present Altitude - 5-1600 m

Collection(s) - Alston 16276; bb 68, 69, 20215, 24066; Bloembergen 4040, 4093, 4165, 4259; Coode 5987, 6017; De Vogel 5638, 5703, 5784, 5831, 5916; De Vogel \& Vermeulen 6809; Hennipman 5791, 5934, 6038; Johansson 112; Meijer 11198, 11511; Prawiroatmodjo \& Soewoko 1690, 1719; Van Balgooy 3117,3629

\section{CLERODENDRUM}

\section{Clerodendrum calamitosum L.}

Sulawesi distribution - South

Collection(s) - Noerkas 2

Clerodendrum chinense (Osbeck) Mabb.

Sulawesi distribution - North, South

Altitude $-200 \mathrm{~m}$

Collection(s) - Koorders 19510; Noerkas 327

Clerodendrum inerme Gaertn.

Vernacular name(s) — Lappang beke; Soesoeninang; Sombat

Sulawesi distribution - North, Central, South, South-East

Altitude $-0-100 \mathrm{~m}$

Collection(s) - Elbert 2539, 2683; Kaudern 201; Lam 2683; Meijer 9277, 10114; Noerkas 403, 409; Rachmat 179, 342

Clerodendrum inerme Gaertn. var. oceanicum Gray

Sulawesi distribution - South-East

Altitude $-0-100 \mathrm{~m}$

Collection(s) - Docters van Leeuwen-Reynvaan 1311; Elbert 2539, 2683

Clerodendrum minahassae Teijsm. \& Binn.

Vernacular name(s) - Soepangka asoewan'a Sulawesi distribution - North, Central, South Altitude - 10-300 m

Collection(s) - Bünnemeyer 10776; Cel V 295; Kaudern 499; Koorders 19517, 19519, 19521, 19523; Lam 3047; Teijsmann HB 5868, HB 12146

\section{Clerodendrum paniculatum L.}

Sulawesi distribution - Central Altitude $-200 \mathrm{~m}$ 
Collection(s) - Ramlanto \& Fanani 468

Clerodendrum puberulum Merr.

Sulawesi distribution - South-East

Altitude $-50-100 \mathrm{~m}$

Collection(s) - Prawiroatmodjo \& Maskuri 1328,1569

\section{Clerodendrum sahelangii Merr.}

Sulawesi distribution - North

Collection(s) - Vorderman 34

Clerodendrum speciosissimum Paxton

Sulawesi distribution - Central

Lore Lindu National Park \& environs - present

Collection(s) - Meijer 9215

\section{Clerodendrum squamatum Hallier}

Sulawesi distribution - South-East

Altitude - 0-200 m

Collection(s) - Elbert 2938, 2994, 2998, 3413

Clerodendrum thomsonae Balf.

Sulawesi distribution - South

Collection(s) - Noerkas 63

Clerodendrum spec.

Sulawesi distribution - North, Central, South, South-East

Altitude - 20-1900 m

Collection(s) - Chin 3467, 3545; De Joncheere 1140; De Vogel 5190, 5463; De Vogel \& Vermeulen 6460; Hennipman 5967; Johansson 392; Prawiroatmodjo \& Soewoko 1623 , 1670; Ramlanto 190; Van Balgooy 3054, 3410, 3593; Yoshida 949

\section{DURANTA}

\section{Duranta repens L.}

Sulawesi distribution - Central

Collection(s) - Kaudern 487

\section{GEUNSIA}

\section{Geunsia apoensis Elmer}

Sulawesi distribution - South

Collection(s) - Noerkas 473, 499; Teijsmann HB 13697, HB 13770

\section{Geunsia cinnamomea Hallier}

Sulawesi distribution - South-East

Altitude - 40-900 m

Collection(s) - Elbert 3096, 3461, 3486

Geunsia cumingiana Rolfe

Sulawesi distribution - South-East

Altitude - 100-150 m

Collection(s) - Prawiroatmodjo \& Maskuri 1102,1532

\section{Geunsia farinosa Blume}

Sulawesi distribution - North, Central, SouthEast

Altitude $-25-800 \mathrm{~m}$

Collection(s) - Elbert 2760, 2999; Kaudern 417; Lam 2523, 2866; Meijer 9413

Geunsia furfuracea (Bakh.) Moldenke

Sulawesi distribution - Central, South, SouthEast

Altitude $-25-1000 \mathrm{~m}$

Collection(s) - bb 11429; Bünnemeyer 11488, 11550; Kjellberg 409; Teijsmann HB 12529; Van Vuuren 425

Geunsia grandiflora Hallier f.

Sulawesi distribution - South

Altitude $-150 \mathrm{~m}$

Collection(s) - Elbert 3204

Geunsia hexandra (Teijsm. \& Binn.) Koord.

Vernacular name(s) - Donawoe; Koenai; Lelema

Sulawesi distribution - North, Central, South, South-East

Altitude - 0-1060 m

Collection(s) - bb 12664, 17114, 21756, 24101; Cel II 337, II 450; Elbert 2690, 2760, 2999, 3040, 3096, 3378; Koorders 19492, 19493, 19495, 19496, 19497, 19533; Prawiroatmodjo \& Maskuri 1329; Ramlanto \& Fanani 463; Van Vuuren 620, 637, 640, 753

\section{Geunsia spec.}

Sulawesi distribution - North, South

Altitude - 100-480 m

Collection(s) - Alston 15763; Cel II 450; Kofman 109, 116 


\section{GMELINA}

\section{Gmelina asiatica L.}

Sulawesi distribution - Central

Lore Lindu National Park \& environs - present Altitude - 0-100 m

Collection(s) - Hennipman 5018; Van Balgooy 2955

\section{Gmelina elliptica Sm. ex Nees}

Vernacular name(s) - Boengango; Wewenganga

Sulawesi distribution - North, Central, South, South-East

Altitude - 0-645 m

Collection(s) - Amir 70; bb 24; Elbert 2619, 3001, 3038, 3058; Kaudern 470; Koorders 19525, 19526, 19527; Lam 2462; Prawiroatmodjo \& Maskuri 1082, 1173; Ramlanto 113; Teijsmann HB 11885, HB 13845, HB 14156; Van Vuuren 112; Widjaja 551

Gmelina palawensis H.J. Lam

Vernacular name(s) - Kajoe poetih; Woeroko Sulawesi distribution - South

Altitude - 25-600 m

Collection(s) - bb 8560, 23574; Cel II 441, III 35, III 168, III 169, IV 128, IV 189

\section{Gmelina spec.}

Sulawesi distribution - North, Central, South Altitude $-0-500 \mathrm{~m}$

Collection(s) - Coode 5908; De Vogel 5707, 6367; Forman 407; Van Balgooy 3797, 3858

\section{LANTANA}

\section{Lantana camara $L$.}

Sulawesi distribution - North, Central, South, South-East

Altitude $-50-950 \mathrm{~m}$

Collection(s) - Bünnemeyer 10562, 10732, 11018; Buwalda 3747; De Vogel \& Vermeulen 6995; Prawiroatmodjo \& Maskuri 1574

\section{PHYLA}

Phyla nodiflora (L.) Greene

Sulawesi distribution - Central
Lore Lindu National Park \& environs — present Altitude $-30 \mathrm{~m}$

Collection(s) - Meijer 9170; Van Balgooy 2984

Phyla nodiflora (L.) Greene forma spathulata Moldenke

Sulawesi distribution - North

Collection(s) - Noerkas 90

\section{PREMNA}

\section{Premna foetida Reinw.}

Sulawesi distribution - Central, South

Altitude $-250-500 \mathrm{~m}$

Collection(s) - Meijer 10202; Ramlanto 237

\section{Premna pubescens Blume}

Sulawesi distribution - South-East

Altitude $-0-200 \mathrm{~m}$

Collection(s) - Elbert 3366

Premna pubescens Blume forma odorata H.J. Lam

Sulawesi distribution - South-East

Altitude - 0-60 m

Collection(s) - Elbert 2811; Teijsmann HB 14141

\section{Premna serratifolia L.}

Vernacular name(s) - Djaroe; Kambarogo; Ojo goum; Saroh; Urego

Sulawesi distribution - North, Central, South Lore Lindu National Park \& environs - present Altitude - 0-1000 m

Collection(s) - Amir 39; bb 7; Bloembergen 4039; Bünnemeyer 10585; Elbert 2624, 2725 , 2804, 2884, 2932; Kaudern 204; Koorders 19535, 24073; Lam 2534; Meijer 10690; Noerkas 405; Prawiroatmodjo \& Soewoko 1861; Teijsmann HB 11959, 18028; Weir 13

\section{Premna tomentosa Willd.}

Sulawesi distribution - North, South-East

Collection(s) - bb 17183, 21326, 21327

\section{Premna spec.}

Sulawesi distribution - North, South, SouthEast

Altitude - 20-400 m 
Collection(s) - Alston 16011, 16210; Chin 3486; De Vogel 5700; Hennipman 5769A, 5770; Prawiroatmodjo \& Soewoko 1967; Yusuf \& Wahyono 3

\section{TEIJSMANNIODENDRON}

\section{Teijsmanniodendron spec.}

Sulawesi distribution - Central

Lore Lindu National Park \& environs - present Altitude $-1100 \mathrm{~m}$

Collection(s) - Keßler et al. PK 3156

\section{VITEX}

Vitex celebica Koord.

Sulawesi distribution - North, South, SouthEast

Collection(s) - bb 5159, 21775; Cel V 249, Cel V 383; Ham R 5; Koorders 19544, 19548, 19554, 19558, 19560; Ramlanto 123; Van Hulstijn 406

\section{Vitex cofassus Reinw.}

Sulawesi distribution - North, Central, South, South-East

Collection(s) - bb 7502, 8579, 13745, 20755, 21984, 23307, 25004, 31521; Beversluis 2; Church \& Ismail 20; McDonald \& Ismail 4039; Meijer 10825; Teijsmann HB 12879; Van Hulstijn 337

Vitex cofassus Reinw. var. cofassus

Sulawesi distribution - South-East

Collection(s) - bb 2239

Vitex glabrata $R$. Br.

Sulawesi distribution - North, South, SouthEast

Collection(s) - bb 6038; Church \& Ismail 28; Ramlanto 118

\section{Vitex longifolia Merr.}

Sulawesi distribution - South

Collection(s) - Teijsmann HB 11788

Vitex parviflora Juss.

Sulawesi distribution - South-East

Collection(s) - Docters van Leeuwen-Reynvaan 1309; Elbert 2732

\section{Vitex pinnata $L$.}

Sulawesi distribution - North, Central, SouthEast

Collection(s) - bb 19394; Elbert 2982; Meijer 10237

Vitex quinata F.N. Williams

Sulawesi distribution - North, Central

Collection(s) - bb 31495; Meijer 9424, 10067

\section{Vitex trifolia L.}

Sulawesi distribution - North

Collection(s) - Meijer 10117

Vitex trifolia L. var. trifolia

Sulawesi distribution - South, South-East

Collection(s) - Bünnemeyer 10633, 10828, 11153, 11234; Docters van Leeuwen-Reynvaan 1710 ; Rachmat 35

\section{Vitex spec.}

Sulawesi distribution - North, Central, South

Collection(s) - Alston 15993; De Joncheere 1018; De Vogel 5020, 5760, 5782, 6218; De Vogel \& Vermeulen 6668,6959 ; Hennipman 5011

\section{VIOLACEAE}

\section{RINOREA}

\section{Rinorea bengalensis (Wall.) Kuntze}

Vernacular name(s) - Waringi

Sulawesi distribution - South, South-East

Altitude $-30 \mathrm{~m}$

Collection(s) - bb 26281; Cel II 459; Teijsmann HB 11987

Rinorea horneri (Korth.) Kuntze

Vernacular name(s) - Nialetta

Sulawesi distribution - North, South

Altitude - $250 \mathrm{~m}$

Collection(s) - Lam 2902; Teijsmann HB 11725

Rinorea javanica (Blume) Kuntze

Sulawesi distribution - North

Altitude $-200 \mathrm{~m}$

Collection(s) - Koorders 16794 


\section{WINTERACEAE}

\section{DRIMYS}

\section{Drimys piperita Hook.f.}

Vernacular name(s) - Kajoe Tambara

Sulawesi distribution - North, Central, South Lore Lindu National Park \& environs - present Altitude - 1500-3000 m

Collection(s) - bb 20554; Bloembergen 3970, 3997; Bünnemeyer 11885, 12049, 12295 , 12323; De Vogel 5138, 5138A, 5139, 5349, 5370, 5374, 5412, 5469; Eyma 469, 631, 904, 1349, 3584, 3772, 3959; Meijer 9824; Rachmat 903 


\section{INDEX}

Index to the scientific names of families and genera. Numbers refer to text pages

A

Abelmoschus

Abroma

Abutilon

Acacia

Acalypha

Acer

Aceraceae

Acmena

Acronychia

Actephila

Actinidiaceae

Actinodaphne

Adenanthera

Adinandra

Adinauclea

Aegiceras

Aegle

Agathis

Aglaia

Aidia

Ailanthus

Alangiaceae

Alangium

Albizia

Alchornea

Alectryon

Aleurites

Allophylus

Alphitonia

Alphonsea

Alseodaphne

Alstonia

Alyxia

Amaracarpus

Ammannia

Anacardiaceae

Anacardium

Anacolosa

Anaxagorea

Anisoptera

Annona

Annonaceae

Anthocephalus

Antiaris

Antidesma

Antirhea

Aphanamixis
Aphananthe

Apocynaceae

Apodytes

Aporosa

Aquifoliaceae

Araliaceae

Araucariaceae

Arcangelisia

Archidendron

Ardisia

Argyreia

Arnicratea

Artabotrys

Artocarpus

Arytera

Ascarina

Asclepiadaceae

Aspidopteris

Astronia

Atuna

Austrobuxus

Averrhoa

Avicennia

Avicenniaceae

Azadirachta

Azima

B

38

128

109

9

61

13

13

113

78

6 Blumeodendron

6 Boehmeria

100 Boenninghausenia

9 Boerhaavia

30 Bombacaceae

9 Bombax

9 Boraginaceae

113 Borreria

88 Brackenridgea

39 Breynia

113 Bridelia

84 Broussonetia
146

135

143

134

111

140

7

18

18

18

68

72

103

28

150

72

17

141

Camellia

Campnosperma

Camptostemon

Cananga

Canarium

Canavalia

Canthium

Capparaceae

Capparis

Caprifoliaceae

40

Capsicum

96 Carallia

66

68

61

17

102

41

Cardiospermum

Caryota

Casearia

Cassia

Cassytha

Castanopsis

Casuarina

Casuarinaceae

Cathormion

Cecropiaceae

Celastraceae

Celastrus

Celtis

Cerbera

Ceriops

Ceriscoides

Chamaesyce

Chamabainia 


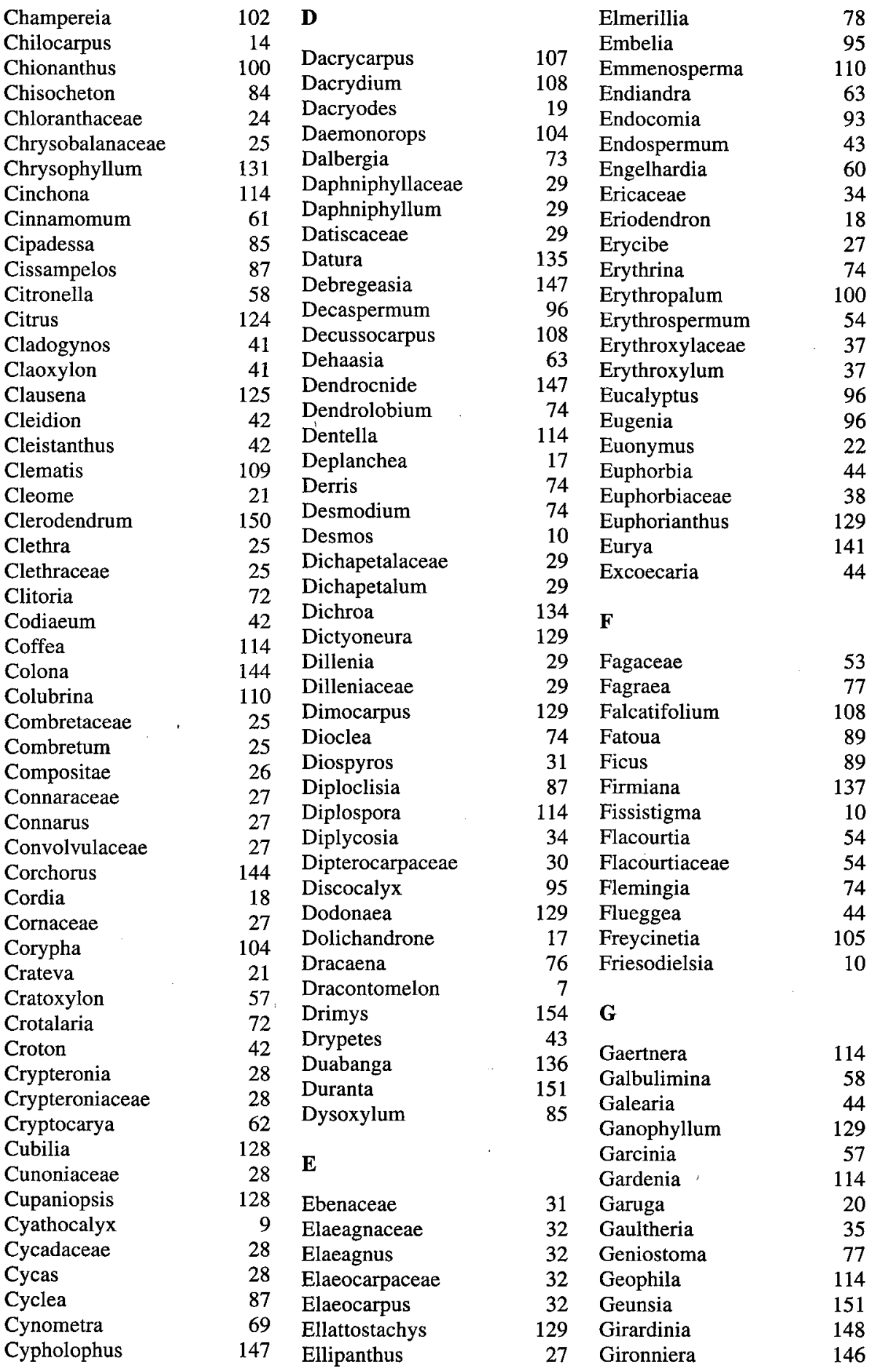




$\begin{array}{lr}\text { Gleditisia } & 7 \\ \text { Gliricidia } & 75 \\ \text { Glochidion } & 4 \\ \text { Gluta } & \\ \text { Glycosmis } & 12 \\ \text { Glyptopetalum } & 23 \\ \text { Gmelina } & 15 \\ \text { Gnetaceae } & 5 \\ \text { Gnetum } & 5 \\ \text { Gomphandra } & 5 \\ \text { Gomphia } & 10 \\ \text { Goniothalamus } & 1 \\ \text { Gonocaryum } & 5 \\ \text { Gonystylus } & 14 \\ \text { Goodeniaceae } & 56 \\ \text { Gordonia } & 142 \\ \text { Gossypium } & 80 \\ \text { Gouania } & 110 \\ \text { Grevillea } & 109 \\ \text { Grewia } & 144 \\ \text { Gronophyllum } & 104 \\ \text { Guettarda } & 114 \\ \text { Guioa } & 129 \\ \text { Guttiferae } & 57 \\ \text { Gymnacranthera } & 93 \\ \text { Gymnostoma } & 22 \\ \text { Gynochthodes } & 114 \\ \text { Gynotroches } & 11 \\ \text { Gyrinops } & 14 \\ & \end{array}$

Hydnophytum

Hypobathrum

Hypserpa

\section{H}

Haematocarpus

Haplolobus

Haplostichanthus

Harpullia

Harrisonia

Hedyosmum

Hedyotis

Helicia

Helicteres

Heliotropium

Heritiera

Hernandia

Hernandiaceae

Hibiscus

Himantandraceae

Hiptage

Homalanthus

Homalium

Homonoia

Hopea

Horsfieldia

Hydnocarpus

\section{I}

Icacinaceae

Ichnocarpus

Ilex

Indigofera

Inocarpus

Intsia

Iodes

Itea

Itoa

Ixonanthaceae

Ixonanthes

Ixora

J

Jasminum

Jatropha

Juglandaceae

\section{$\mathbf{K}$}

Kadsura

Kalappia

Kibara

Kibatalia

Kjellbergiodendron

Kleinhovia

87 Knema

\section{0 \\ Knoxia}

Koordersiodendron

Kopsia

Korthalsia

Kostermanthus

$\mathbf{L}$

Lagerstroemia

Lansium

Lantana

Lasianthus

Lasiococca

Lauraceae

Lawsonia

Lecanthus

Lecythidaceae

Leea

Leeaceae

Leguminosae

Leguminosae-Caesalpinioideae
115 Leguminosae-Mimo-

115 soideae 70

87 Leguminosae-Papilionoideae

Lepidopetalum

Lepiniopsis

Lepisanthes

Lepistemon

Leptospermum

Leucaena

Leucosyke

Licania

Licuala

Ligustrum

Liliaceae

Lindera

Litchi

Lithocarpus

Litsea

Livistona

101 Loeseneriella

47 Loganiaceae

60 Lophopetalum

Lucinaea

Lumnitzera

Lunasia

134
70 Lycianthes

Lythraceae

14

130

27

97

71

148

25

104

102

76

63

130

53

64

105

23

77

23

117

25

125

135

78

14

97
138

M

138 Macadamia

Macaranga

116 Maclura 92

7 Macropsychanthus $\quad 75$

14 Madhuca 131

104 Maesa 95

25 Magnolia 79

Magnoliaceae $\quad 78$

Malaisia $\quad 92$

78 Mallotus 49

86 Malphighiaceae 79

152 Malvaceae 79

116 Mangifera 7

47 Manglietia 79

60 Manihot 50

78 Manilkara 131

148 Maniltoa 70

66 Maranthes 25

67 Maschalocorymbus $\quad 117$

67 Mastersia $\quad 75$

68 Mastixia 27

Medinilla $\quad 81$

68 Medusanthera 59 
Melanolepis

Melastoma

Melastomataceae

Melia

Meliaceae

Melicope

Meliosma

Melochia

Melodinus

Memecylon

Menispermaceae

Mesua

Metrosideros

Michelia

Microcos

Micromelum

Millettia

Millingtonia

Mimosa

Mimusops

Miquelia

Mischocarpus

Mitrephora

Monimiaceae

Moraceae

Morinda

Moringa

Moringaceae

Morus

Mucuna

Murraya

Mussaenda

Mussaendopsis

Mycetia

Myrica

Myricaceae

Myristica

Myristicaceae

Myrmecodia

Myrmephytum

Myrsinaceae

Myrtaceae

Myxopyrum

$\mathbf{N}$

Nauclea

Neolitsea

Neonauclea

Neoscortechinia

Neotrewia

Nephelium

Nertera

$\begin{aligned} 50 & \text { Neuburgia } \\ 82 & \text { Nothaphoebe } \\ 81 & \text { Nothocnide } \\ 86 & \text { Nyctaginaceae } \\ 82 & \\ 125 & \text { O }\end{aligned}$

127

138

14

82

87

58

97

79

145

126

75

17

71

132

59

130

11

88

88

117

92

92

92

76

126

118

118

118

93

93

93

93

118

118

94

96

102

Ochnaceae

Octomeles

Olacaceae

Olax

Oldenlandia

Oleaceae

Omphalea

Oncosperma

Opilia

Opiliaceae

Oreocnide

Ormosia

Orophea

Oroxylum

Osbeckia

Osmelia

Osmoxylon

Oxalidaceae

P

Pachygone

Pachystylidium

Paederia

Palaquium

Palmae

Pandanaceae

Pandanus

Pandorea

Pangium

Parasponia

Parkia

Parsonsia

Pavetta

Pellacalyx

Peltophorum

Pemphis

Pericampylus

Perrottetia

119

65

119

50

51

130

120
Persea

Phaeanthus

Phaleria

Phoebe

Pholidocarpus

Phyla

Phyllanthus
77

65

148

99

100

29

100

100

120

100

51

105

102

102

149

76

11

17

82

55

16

102

\section{Popowia}

Porterandia

Pouteria

Pouzolzia

Praravinia

Premna

Procris

Proteaceae

Prumnopitys

Prunus

Pseudoclausena

Pseuduvaria

Psidium

Psychotria

Psydrax

Pternandra

Pterocarpus

Pterocymbium

Pterospermum

Pterygota

Pueraria

Pycnarrhena

Pyrenaria

143

66

105

152

51

$Q$

Quassia

Quisqualis
108

136

59

135

105

149

52

105

149

99

72

107

107

133

67

107

107

107

108

22 
$\mathbf{R}$

Rademachera

Randia

Ranunculaceae

Rapanea

Rauvolfia

Reinwardtiodendron

Reissantia

Rhamnaceae

Rhizophora

Rhizophoraceae

Rhododendron

Rhus

Rhysotoechia

Ricinus

Rinorea

Rosaceae

Rothmannia

Rourea

Rubiaceae

Rutaceae

Ryparosa

Ryssopteris

\section{S}

Sabia

Sabiaceae

Sageraea

Salacia

Salvadoraceae

Sambucus

Sandoricum

Santalaceae

Santalum

Santiria

Sapindaceae

Sapotaceae

Saprosma

Saraca

Sarcandra

Sarcosperma

Sarcotheca

Saurauia

Sauropus

Saxifragaceae

Scaevola

Schefflera

Schima

Schisandraceae

Schleichera

Schradera
Schuurmansia

Scolopia

Scyphiphora

Semecarpus

Senna

Serianthes

Shorea

Simaroubaceae

Sindora

109

111

111

153

112

121$$
5
$$

52 Tabernaemontana

134 Tarenna

56 Tarennoidea

16 Taxaceae

142 Taxus

134 Teijsmanniodendron

131 Tephrosia

121 Terminalia
100 Ternstroemia

55 Tetracera 30

121 Tetractomia 126

8 Tetradium 126

70 Tetrameles 29

72 Theaceae 141

30 Thespesia 81

134 Thymelaeaceae 143

70 Tiliaceae 143

24 Timonius 122

34 Tinospora 88

135 Toddalia 126

136 Toona 86

136 Tournefortia 18

136 Trema 146

76 Triadica 52

52 Tricalysia 123

76 Trichadenia 55

122 Trichospermum 145

137 Trigonachras 131

137 Trigonobalanus 54

122 Trigonopleura 53

8 Trigonostemon 53

137 Tristaniopsis 99

60 Tristira 131

87 Tristiropsis 131

139 Triumfetta 145

137 Trophis 92

92 Turnera 146

100 Turneraceae 146

76 Turpinia 137

15 Turraea 86

52

78 U

140 Ulmaceae 146

140 Uncaria 123

52 Urophyllum 123

30 Urticaceae 147

52 Uvaria 12

140

$140 \mathrm{~V}$

98 Vaccinium 36

Vatica 30

Ventilago $\quad 110$

15 Verbenaceae 150

122 Vernonia 26

122 Viburnum 21

141 Vigna $\quad 76$

141 Villebrunea $\quad 149$

153 Violaceae 153

76 Vitex 153

26 Voacanga 15 
$\mathbf{w}$

Wallaceodendron

Walsura

Weinmannia

Wendlandia

Wikstroemia

Winteraceae

Wrightia
$\mathbf{X}$

72 Xanthomyrtus

86 Xanthophyllum

28 Xanthostemon

124 Ximenia

143 Xylocarpus

154 Xylopia

15 Xylosma
$\mathbf{Z}$

99 Zanthoxylum

127

109 Zizyphus

99 Zornia

100

86

12

55 